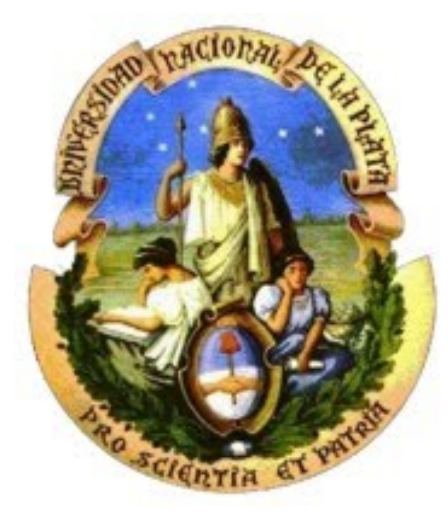

UNIVERSIDAD NACIONAL DE LA PLATA FACULTAD DE CIENCIAS EXACTAS DEPARTAMENTO DE FÍSICA

Tesis presentada para optar al grado de Doctor de la Facultad de Ciencias Exactas

\title{
Estudio holográfico de la estructura hadrónica
}

Mg. Leandro Ezequiel Koile

Doctorando

Dr. Martín Schvellinger

Director

Año 2015 


\title{
Resumen
}

En esta Tesis se realiza un estudio pormenorizado de la dispersión inelástica profunda $(D I S)$ de leptones por mesones para acoplamiento fuerte y en el límite planar a partir de modelos duales basados en la correspondencia $A d S / C F T$. Se estudia la estructura de mesones escalares y mesones vectoriales en su polarización más general, con grados de libertad de sabor en el contexto de los modelos de D3D7-branas en la teoría de supercuerdas del tipo IIB y de D4D8 $\overline{\mathrm{D} 8}$ y D4D6 $\overline{\mathrm{D} 6}$-branas en la teoría de supercuerdas del tipo IIA. El primer caso se trata de una teoría de gauge en $3+1$ dimensiones que preserva $\mathcal{N}=2$ supersimetrías, mientras que los últimos dos corresponden a teorías de Yang-Mills no supersimétricas. Se estudian los límites del parámetro de Bjorken $x \lesssim 1$ (estrictamente $\frac{1}{\sqrt{\lambda}} \ll x<1$, donde $\lambda$ es el acoplamiento de 't Hooft), que requiere de una descripción de supergravedad, y el límite $x \ll 1$ (estrictamente $e^{-\sqrt{\lambda}} \lesssim x \ll \frac{1}{\sqrt{\lambda}}$ ), para el cual los cálculos se deben hacer mediante la teoría de cuerdas. También se estudia la sección eficaz diferencial de dispersión en diversos regímenes de polarización en estos modelos. Se encuentran importantes relaciones entre las distintas funciones de estructura en cada régimen del parámetro $x$ para los distintos modelos estudiados, lo cual sugiere un comportamiento universal.

\begin{abstract}
In this Thesis we carry out a detailed study of Deep Inelastic Scattering (DIS) of leptons from mesons in the strongly coupled regime and in the planar limit from dual models arising from the $A d S / C F T$ correspondence. We study the structure of scalar and vector mesons in their most general polarization state, with flavor degrees of freedom in the context of the D3D7-brane model in Type IIB Superstring Theory, and the D4D8 $\overline{\mathrm{D} 8}$ and $\mathrm{D} 4 \mathrm{D} 6 \overline{\mathrm{D} 6}$ models in Type IIA Superstring Theory. The first model corresponds to a $3+1$ dimensional gauge theory preserving $\mathcal{N}=2$ supersymmetries while the last two models correspond to non-supersymmetric YangMills gauge theories. We study the limits of the Bjorken parameter $x \lesssim 1$ (strictly $\frac{1}{\sqrt{\lambda}} \ll x<1$, being $\lambda$ the 't Hooft coupling), which requires a supergravity description and the limit $x \ll 1$ (strictly $e^{-\sqrt{\lambda}} \lesssim x \ll \frac{1}{\sqrt{\lambda}}$ ), where calculations are performed by using String Theory. We also study the DIS differential cross section for different polarization regimes in these models. We find important relations among the structure functions in each regime of the parameter $x$ for the models studied, which suggests a universal behavior.
\end{abstract}




\section{Publicaciones}

Las contribuciones originales de esta tesis están basadas en los siguientes trabajos:

[1] E. Koile, S. Macaluso and M. Schvellinger, "Deep Inelastic Scattering from Holographic Spin-One Hadrons," JHEP 1202, 103 (2012) [arXiv:1112.1459 [hep-th]]. 37 pp.

2 [2] E. Koile, S. Macaluso and M. Schvellinger, "Deep inelastic scattering structure functions of holographic spin-1 hadrons with $N_{f} \geq 1$," JHEP 1401, 166 (2014) [arXiv:1311.2601 [hep-th]]. $41 \mathrm{pp}$.

[3] E. Koile, N. Kovensky and M. Schvellinger, "Hadron structure functions at small $x$ from string theory," JHEP 1505, 001 (2015) [arXiv:1412.6509 [hep-th]].

[4] E. Koile, N. Kovensky and M. Schvellinger, "Deep inelastic scattering cross section from the gauge/string duality". Trabajo en preparación. 


\section{Índice general}

Resumen I

1. Introducción e idea general 1

I Base teórica 4

2. Teorías cuánticas de campos en el límite planar y correspondencia $A d S / C F T \quad \mathbf{5}$ 2.1. Teorías de Yang-Mills en cuatro dimensiones en el límite planar . . . . . . . . 7 2.2. La correspondencia $A d S / C F T \ldots \ldots \ldots \ldots \ldots$

3. Amplitudes de dispersión en teoría de cuerdas: construcción de lagrangianos $\begin{array}{ll}\text { efectivos } & 10\end{array}$

3.1. Método para calcular lagrangianos efectivos en la teoría de cuerdas . . . . . . . . 10

3.2. Amplitudes de dispersión en espacio curvo . . . . . . . . . . . . . . . . 13

$\begin{array}{ll}\text { 4. Dispersión inelástica profunda (DIS) } & 15\end{array}$

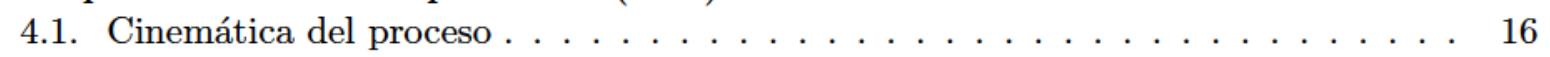

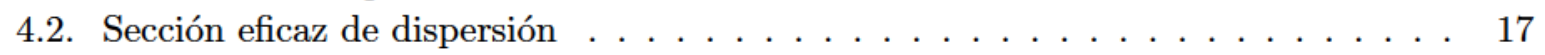

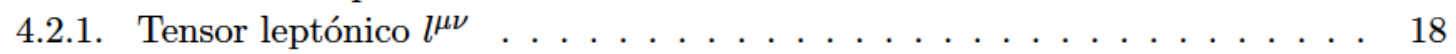

4.2 .2 . Tensor hadrónico $W_{\mu \nu} \ldots \ldots \ldots \ldots \ldots$. . . . . . . . . . . 19

4.3. Funciones de estructura del hadrón . . . . . . . . . . . . . . . . . . . 20

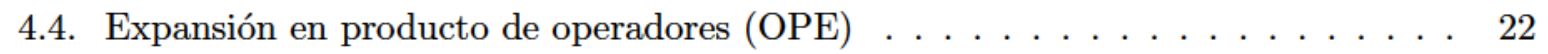

5. Modelos holográficos duales de teorías de YM confinantes 25

5.1. Modelo holográfico de Polchinski y Strassler . . . . . . . . . . . . . . . 25

5.2. Modelos con branas de sabor . . . . . . . . . . . . . . . . . . . . . . 28

$5.2 .1 . \quad$ Modelo D3D7 . . . . . . . . . . . . . . . . . . . . . . . . 29

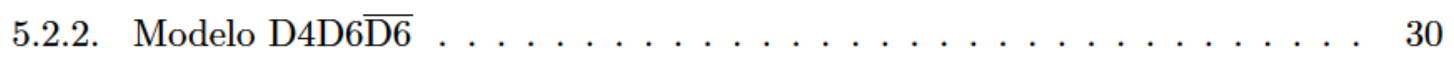

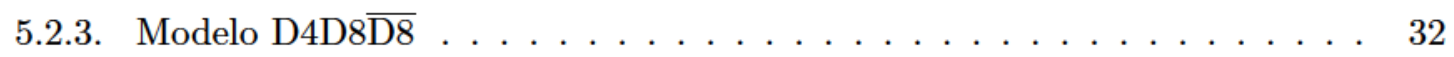

II Contribuciones originales 34

\begin{tabular}{|l|l|}
\hline 6. Estudio del caso $x \lesssim 1$ : régimen de supergravedad & 35
\end{tabular}

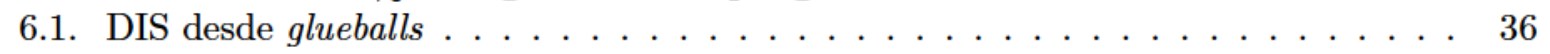

6.2. DIS desde mesones escalares . . . . . . . . . . . . . . . . . . . . . . 46

6.3. DIS desde mesones vectoriales . . . . . . . . . . . . . . . . . . . . 50

6.4 . Extensión a modelos más generales . . . . . . . . . . . . . . . . 56

6.4.1. DIS desde mesones escalares para modelos más generales . . . . . . . . 57 
6.4.2. DIS desde mesones vectoriales para modelos más generales . . . . . . . . 61

6.5. DIS desde mesones vectoriales con $N_{f}>1 \ldots \ldots \ldots \ldots \ldots$. . . . . . . 64

6.6. Contribuciones subdominantes en las expansiones $\frac{1}{N}$ y $\frac{N_{f}}{N} \ldots \ldots \ldots$. . . . . . . 68

6.7. Discusión de los resultados . . . . . . . . . . . . . . . . 82

7. Estudio del caso $x \ll 1:$ régimen de teoría de cuerdas $\quad 84$

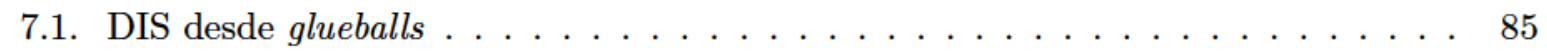

7.1.1. Amplitud de dispersión a partir de la teoría de cuerdas . . . . . . . . . . 88

7.1.2. Amplitud de dispersión a partir de supergravedad . . . . . . . . . . . . 91

7.1.3. Lagrangiano efectivo . . . . . . . . . . . . . . . . . . 94

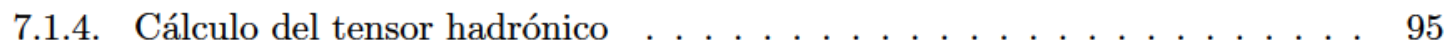

7.2. DIS desde mesones escalares . . . . . . . . . . . . . . . . . . . . . . . 98

7.2.1. Amplitud de dispersión de cuatro puntos para cuerdas abiertas y cerradas 99

7.2.2. Amplitud de dispersión a partir de supergravedad . . . . . . . . . . . 103

7.2.3. Lagrangiano efectivo . . . . . . . . . . . . . . . . . . . 106

7.2.4. Cálculo del tensor hadrónico ． . . . . . . . . . . . . . . . . 107

7.3. DIS desde mesones vectoriales . . . . . . . . . . . . . . . . . . 110

7.3.1. Amplitud de dispersión de cuatro puntos para cuerdas abiertas y cerradas 111

7.3.2. Amplitud de dispersión a partir de supergravedad . . . . . . . . . . . . 112

$7.3 .3 . \quad$ Lagrangiano efectivo . . . . . . . . . . . . . . . . . . . . . . 115

7.3.4. Cálculo del tensor hadrónico . . . . . . . . . . . . . . . . . 116

7.4. Extensión a fondos más generales ～. . . . . . . . . . . . . . . . 120

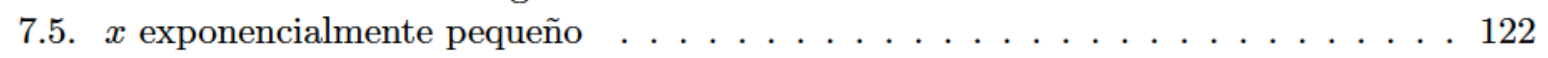

7.6. Discusión de los resultados . . . . . . . . . . . . . . . . . . . . 125

\begin{tabular}{ll}
\hline 8. Secciones eficaces diferenciales & 127
\end{tabular}

8.1. Generalidades . . . . . . . . . . . . . . . . . . . . . . 127

8.2. Sección eficaz de DIS y funciones de estructura: Caso general . . . . . . . . . . 128

8.2.1. Polarización arbitraria . . . . . . . . . . . . . . . . . . 128

8.2.2. Estudio de las polarizaciones del hadrón . . . . . . . . . . . . . . . . . 131

8.2.3. Amplitudes de helicidad . . . . . . . . . . . . . . . . . . . . . . . . 134

8.3. Sección eficaz y funciones de estructura: Resultados holográficos . . . . . . . . . 135

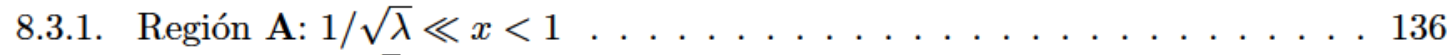

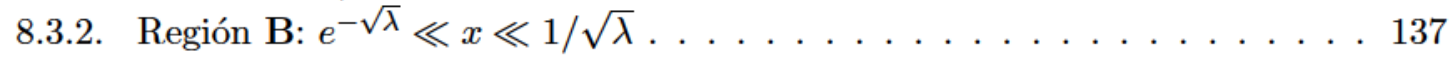

8.4. Discusión de los resultados . . . . . . . . . . . . . . . . . . . . . . 138

\begin{tabular}{ll}
\hline 9. Conclusiones & 147
\end{tabular}

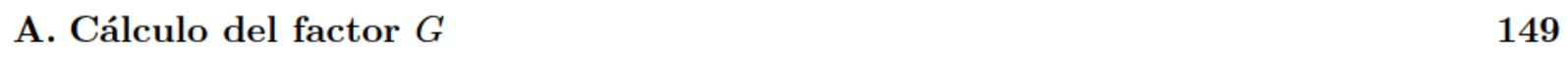

\begin{tabular}{ll}
\hline Agradecimientos & 158
\end{tabular} 


\section{Capítulo 1}

\section{Introducción e idea general}

El objetivo de esta Tesis es el de estudiar el comportamiento de teorías de Yang-Mills (en adelante YM) confinantes en cuatro dimensiones en el límite de acoplamiento fuerte, con el fin de estudiar la física de hadrones en este rango. La teoría que mejor describe la física de la interacción fuerte es la cromodinámica cuántica (QCD, del inglés Quantum ChromoDynamics) que, sin embargo, pierde su poder predictivo al considerar problemas en el límite de acoplamiento fuerte, el cual es un límite no perturbativo de la teoría cuántica de campos.

En esta Tesis utilizamos la correspondencia $A d S / C F T$ para estudiar propiedades de hadrones en ciertos modelos holográficos duales derivados de la teoría de supercuerdas, cuyas teorías de gauge asociadas comparten importantes aspectos con QCD en el régimen de acoplamiento fuerte en el límite planar. En particular, en dichos modelos se obtienen mesones holográficos. Específicamente, estudiamos el problema de la dispersión inelástica profunda (en adelante DIS, del inglés Deep Inelastic Scattering) de leptones por dichos mesones holográficos, con el objetivo de calcular el tensor hadrónico de estos últimos, cuya estructura se describe en función de las llamadas funciones de estructura. El problema de calcular este tensor no puede encararse en términos de QCD perturbativa, dado que depende de dinámica suave (soft) de QCD.

Polchinski y Strassler [5] desarrollaron una propuesta para calcular el tensor hadrónico para glueballs utilizando la correspondencia $A d S / C F T{ }^{1}$ Obtuvieron las funciones de estructura para glueballs en una deformación del límite de $N$ grande de la teoría $S U(N) \mathcal{N}=4$ Súper Yang- Mills (en adelante SYM), conocida como $\mathcal{N}=1^{*} \mathrm{SYM}$ [11]. Estos glueballs, singletes de color, están formados por gluones que transforman en la representación adjunta del grupo de gauge. En este trabajo extenderemos y aplicaremos este método sistemáticamente a modelos más complejos con los que estudiaremos DIS por mesones escalares y vectoriales con una polarización arbitraria. Estos están formados por quarks y antiquarks, que transforman en la representación fundamental del grupo de gauge. Centraremos nuestro análisis en tres modelos [12, 13, 14] derivados directamente desde teorías de supercuerdas. Todos estos poseen como modelos holográficos duales sendas teorías cuánticas de campos con grandes similitudes a QCD, como ya hemos comentado. Este procedimiento es válido para el régimen fuertemente acoplado de la teoría de gauge, es decir, cuando el parámetro de 't Hooft $\lambda=g_{Y M}^{2} N=g_{s} N$ cumple $1 \ll \lambda \ll N 2$.

En la Figura 1.1 pueden verse los distintos regímenes para los que puede analizarse el DIS en

\footnotetext{
${ }^{1}$ En referencia a la descripción de glueballs en $A d S / C F T$ ver [6, 7, 8, 19, 10].

${ }^{2}$ Esta es la condición para el caso D3D7, que corresponde a supergravedad de tipo IIB. Para los modelos D4D8 $\overline{\mathrm{D} 8}$ y $\mathrm{D} 4 \mathrm{D} 6 \overline{\mathrm{D} 6}$ la relación correcta, proveniente de supergravedad de tipo IIA, es $1 \ll \lambda U \ll N^{\frac{4}{3}}$, donde $U$ es la coordenada radial [15]. Durante esta Tesis utilizaremos en general la relación válida para el modelo D3D7, que es en el que realizaremos los cálculos en mayor detalle, pero debemos tener en cuenta cómo se modifica esta relación para los otros modelos considerados.
} 


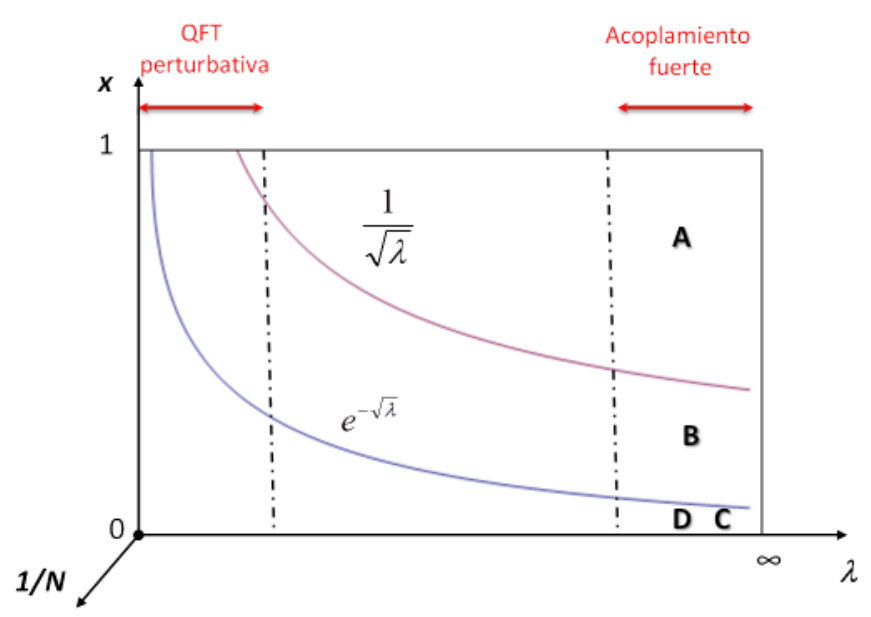

Figura 1.1: Ilustración esquemática del espacio de parámetros para el problema de DIS. Estudiaremos en detalle las regiones A, B y C.

términos del parámetro de 't Hooft $\lambda$ y el parámetro $x$ de Bjorken. Todo este análisis se hace con $N \rightarrow \infty$ fijo, por lo que la expansión en $\frac{1}{N}$ correspondería a extenderse en el eje perpendicular al plano del gráfico. Estudiaremos el caso de acoplamiento fuerte y $N$ grande $1 \ll \lambda=g_{s} N \ll N$, donde $g_{s}$ es la constante de acoplamiento de la teoría de cuerdas, que se divide en las regiones A, B, C y D en notación de [5] . La región A corresponde al límite en el cual el parámetro de Bjorken es relativamente grande, concretamente, el rango para el cual $\frac{1}{\sqrt{\lambda}} \ll x<1$, siendo $x=1$ el caso de dispersión elástica. En esta región el modelo dual es el de supergravedad y será el objeto del Capítulo 6. El segundo régimen cinemático (región $\mathbf{B}$ ) es el que corresponde a $e^{-\sqrt{\lambda}} \ll x \ll \frac{1}{\sqrt{\lambda}}$, caso en el que la descripción holográfica dual corresponde a cuerdas excitadas. Este se estudiará en detalle en el Capítulo 7. En el tercer régimen (región $\mathbf{C}$ ) consideramos valores exponencialmente pequeños de $x \sim e^{-\sqrt{\lambda}}$, correspondientes al caso en el que el tamaño de las cuerdas es comparable con la escala $R$ del $A d S_{5}$. Esta región es interesante pues la interacción no es local, sino que aparece un efecto debido al crecimiento de las cuerdas, el que se estudia en términos de un operador de difusión. Hay un cuarto régimen (región $\mathbf{D}$ ), correspondiente a $\left(\frac{\Lambda}{q}\right)^{\sqrt{\lambda}}<x \ll e^{-\sqrt{\lambda}}$, donde $q$ es el momento transferido por el fotón virtual y $\Lambda$ la escala de confinamiento IR. En esta región se puede utilizar el grupo de renormalización en la hoja de mundo para incluir el efecto del crecimiento de las cuerdas. En líneas generales, siempre que estudiemos el límite planar de la teoría de gauge fuertemente acoplada estaremos considerando la dispersión de un leptón por un hadrón entero y en tal caso no podemos considerar el modelo de partones para describir el DIS.

Para las regiones A, B y $\mathbf{C}$ se obtienen las funciones de estructura de manera analítica en todos los modelos holográficos, tanto para los mesones escalares como para los mesones vectoriales polarizados. En particular, esta es la primera vez que se consiguen calcular de manera analítica las ocho funciones de estructura para mesones vectoriales polarizados a partir de modelos holográficos duales. Más aún, se encuentran nuevas relaciones del tipo Callan-Gross, válidas para todos los modelos holográficos. En particular, para mesones vectoriales se encuentra la relación $F_{2}=2 F_{1}$ en la región $\mathbf{A}$ y $F_{2}=2 x \alpha F_{1}$ en las regiones $\mathbf{B}$ y $\mathbf{C}$, con $1 \leq \alpha \leq 2$ un parámetro dependiente de la dimensión de escala $\Delta$ de cada mesón particular. La similitud en la estructura del tensor hadrónico, así como la independencia de las relaciones de Callan-Gross con respecto al modelo dan la idea de un comportamiento universal que, al cumplirse en un rango tan amplio de teorías de gauge, nos lleva a postular que debería valer también para QCD, al menos en el 
límite planar y en el régimen de acoplamiento fuerte $\lambda \gg 1$.

Finalmente, se calculan las secciones eficaces del proceso de DIS para distintos estados de polarización y se estudian sus consecuencias fenomenológicas.

La Primera Parte de esta Tesis (Capítulos 2 a 5) está destinada a revisar los temas teóricos relevantes para la ulterior descripción del trabajo realizado. En el Capítulo 2 se da una breve introducción al desarrollo de 't Hooft de las teorías de gauge $S U(N)$ fuertemente acopladas en el régimen $1 \ll \lambda \ll N$ y a la correspondencia $A d S / C F T$. En el Capítulo 3 estudiamos la forma en que se calculan las amplitudes de dispersión en teoría de cuerdas a nivel perturbativo y describimos un método para construir lagrangianos efectivos para obtener estas amplitudes. En el Capítulo 4 describimos el proceso físico de la dispersión inelástica profunda DIS. En el Capítulo 5, finalmente, comentaremos las características generales de los modelos holográficos que utilizaremos.

En la Segunda Parte de esta Tesis (Capítulos 6 a 9) se describirán de manera ordenada los resultados obtenidos durante los años de trabajo en esta área, contenido publicado en los trabajos [1], 2], 3] y [4. En el Capítulo 6 estudiaremos el proceso de DIS en los modelos con grados de libertad de sabor en la región $\mathbf{A}$ descripta más arriba, que corresponde al régimen de supergravedad. En el Capítulo 7 estudiaremos los mismos modelos en las regiones B y $\mathbf{C}$, correspondientes a teoría de cuerdas perturbativa. En el Capítulo 8 calcularemos las secciones eficaces de dispersión para el caso general de estos modelos, así como para algunas polarizaciones específicas. Finalmente, en el Capítulo 9 reseñaremos las conclusiones del trabajo en líneas generales. 
Parte I

\section{Base teórica}




\section{Capítulo 2}

\section{Teorías cuánticas de campos en el límite planar y correspondencia $A d S / C F T$}

\section{Teorías de cuerdas}

La teoría cuántica de campos (QFT, del inglés Quantum Field Theory) describe con gran precisión la física de altas energías de las partículas, pero no logra explicar la naturaleza cuántica de la gravedad. En la búsqueda de una teoría consistente para la gravedad cuántica nos encontramos con que los objetos fundamentales no pueden ser partículas puntuales sino objetos extendidos en una dimensión o cuerdas. Estas cuerdas se manifiestan como partículas puntuales localizadas para un observador de bajas energías, y la masa y características de estas partículas efectivas dependerán simplemente del estado de oscilación de estas cuerdas fundamentales. Toda teoría de cuerdas incluye una "partícula" no masiva de espín 2, que representa el gravitón, dando lugar a una teoría cuántica de la gravedad. Encontramos que las teorías de supercuerdas resultan consistentes para $D=10$ dimensiones. Estas suelen estudiarse descomponiendo su espacio en la forma $\mathbb{M}^{4} \times \mathcal{M}_{6}$ donde $\mathbb{M}^{4}$ es el espacio de Minkowski en cuatro dimensiones y $\mathcal{M}_{6}$ alguna variedad compacta en seis dimensiones. Las interacciones de bajas energías en la teoría efectiva estarán determinadas por la geometría de esta variedad, y se espera que alguna de estas numerosas soluciones dé lugar al Modelo Estándar de Física de Partículas o a alguna de sus extensiones supersimétricas.

Si bien hoy se concibe a la teoría de cuerdas como una teoría unificadora de la física, su origen histórico es algo distinto. En la década de 1960 se requería una teoría que explicara la existencia de una gran cantidad de hadrones descubiertos experimentalmente. Se quiso entender a estos hadrones como los distintos modos de rotación de una cuerda, logrando por ejemplo ajustar la relación que da la masa $m$ del hadrón más liviano con un dado espín $J$, a saber $J=\alpha^{\prime} m^{2}$, con $\alpha^{\prime} \sim 1(\mathrm{GeV})^{-2}$. Esta relación, llamada trayectoria de Regge y comprobada para valores de espín hasta $J=\frac{11}{2}$, es la misma que aquella entre la masa y el momento angular de una cuerda rotante relativista de tensión $\left(\alpha^{\prime}\right)^{-1}$. Años después, a principios de la década de 1970, se descubriría que este no es el modelo correcto, sino que los hadrones se describen satisfactoriamente con QCD.

\section{QCD y dualidades}

QCD es la teoría de gauge correspondiente al grupo de simetría local $S U(3)$. Es asintóticamente libre, lo que significa que su constante de acoplamiento $g_{Q C D}$ tiende a cero para energías $\mu$ tendiendo a infinito, y se vuelve fuertemente acoplada para bajas energías, de lo que resulta 
una teoría no perturbativa en este rango. Existen diversas técnicas para enfrentarse con este problema, vale decir, el de estudiar QCD en el límite no perturbativo. Un método consiste en trabajar con modelos efectivos de interacciones que reproduzcan la fenomenología de QCD en el rango de interés [16, 17, 18, 19, 20, 21]. Estos modelos son generalmente analíticos, pero tienen la desventaja de no provenir de primeros principios.

Otro enfoque usual [22] es el de QCD en la red (lattice $Q C D$ ). Este es un enfoque de primeros principios, dado que parte de la teoría completa de QCD, pero es de caracter numérico, con lo que no es tan sencillo estudiar dependencias en los parámetros del problema. Una tercera idea surge de la denominada conjetura de Maldacena o correspondencia AdS/CFT [23, 24, 25], y es la que utilizaremos en este trabajo. En este contexto, podemos encontrar soluciones analíticas y de primeros principios para ciertas teorías de gauge en el acoplamiento fuerte.

Este último método se origina en parte con el trabajo seminal [26] de Gerard 't Hooft en 1974. En este trabajo se propuso un método para resolver teorías como QCD en el rango no perturbativo siempre y cuando el número de "colores" presente -el rango del grupo de gauge $S U(N)$ - sea grande, es decir $N \gg 1$. Estas son las llamadas teorías de gauge de $N$ grande (large $N$ ), y dado que de los diagramas de doble línea de dicha teoría solo contribuyen los planares, este límite $N \rightarrow \infty$ se conoce como el límite planar de la teoría de Yang-Mills. El eje de esta idea es que los observables o funciones de Green de una teoría exacta para $N \rightarrow \infty$ puedan expandirse en potencias de $\frac{1}{N}$. Por otro lado, como explicaremos en la próxima Sección, la expansión en diagramas de la teoría de campos sugiere que para el rango de $N$ grande esta coincide con una teoría de cuerdas libre de constante de acoplamiento $\frac{1}{N}$.

\section{La dualidad gauge/gravedad}

Esta relación entre teorías de YM en el límite planar y teorías de cuerdas es bastante general. En particular, vale para teorías conformes (donde la constante de acoplamiento $g_{Y M}$ es independiente de la energía del proceso). Un ejemplo importante de teoría de YM conforme es el de una teoría supersimétrica con grupo de gauge $S U(N)$ y con cuatro supersimetrías. Esta teoría se conoce como $\mathcal{N}=4$ Súper Yang-Mills $(\mathcal{N}=4 S Y M)$. Esta teoría es maximalmente supersimétrica, es decir, tiene la mayor cantidad posible de cargas de supersimetría en cuatro dimensiones. Contiene gluones (campo de gauge) $A_{\mu}^{a}\left(a=1, \ldots, N^{2}-1 ; \mu=0, \ldots, 3\right)$, así como cuatro fermiones $\lambda_{\alpha}(\alpha=1, \ldots, 4)$ y seis campos escalares reales $\Phi_{I}(I=1, \ldots, 6)$ que transforman en la representación adjunta del grupo de gauge. Estos campos constituyen el supermultiplete de gauge en la teoría de $\mathcal{N}=4 \mathrm{SYM}$.

El lagrangiano de esta teoría queda completamente determinado por la supersimetría: incluye una simetría global $S U(4)_{R}$ que es un automorfismo en el espacio de las supercargas, así como también un grupo de simetría conforme $S O(4,2)$ que incluye las transformaciones de Poincaré y las transformaciones de escala y conformes especiales. La forma de reflejar estas simetrías en la teoría dual de cuerdas es tomarlas como isometrías. Esto nos lleva a un espacio Anti-de Sitter de cinco dimensiones $A d S_{5}$ debido a las simetrías del grupo conforme $S O(4,2)$ y a una esfera pentadimensional $S^{5}$ debido a la simetría $R$ global: $S U(4) \simeq S O(6)$. Con esto concluimos, desde el punto de vista de las simetrías del problema, que el modelo holográfico dual a la teoría cuántica de campos $\mathcal{N}=4 S U(N)$ sería dual a una teoría de supercuerdas del tipo IIB en un espacio $A d S_{5} \times S^{5}$. Esta es la idea subyacente a la llamada correspondencia $A d S / C F T$, que describiremos más adelante en este Capítulo.

El principal problema de este método, con el que cálculos perturbativos en una teoría de cuerdas dan lugar a resultados de cálculos no perturbativos en una teoría de gauge dual, es el hecho de que este método requiere conocer la teoría de cuerdas dual a QCD. Esta teoría no se ha conseguido formular aún; sin embargo, existen diversos modelos [12, 13, 14] de teorías de 
cuerdas cuyos duales, si bien no son exactamente QCD, poseen características similares a esta teoría en distintos aspectos.

\subsection{Teorías de Yang-Mills en cuatro dimensiones en el límite planar}

La teoría que describe correctamente la interacción fuerte, i.e. QCD, es muy útil para describir procesos de altas energías (acoplamiento débil, rango perturbativo) pero se torna técnicamente inmanejable para procesos de bajas energías (acoplamiento fuerte, rango no perturbativo). Ejemplos de fenómenos importantes en este último rango son el confinamiento y la ruptura de la simetría quiral.

Es necesario entonces algún esquema de aproximación, lo que requiere un parámetro de expansión. A simple vista no hay ningún parámetro obvio de expansión presente en las teorías de Yang-Mills en cuatro dimensiones (la constante de acoplamiento $g_{Y M}$ es absorbida en la definición de la escala de confinamiento $\Lambda_{Q C D}$ al considerar el grupo de renormalización), pero 't Hooft propuso [26] que puede utilizarse el parámetro $\frac{1}{N}$, siendo $N$ el rango del grupo de gauge $S U(N)$. El objetivo es entonces resolver la teoría exactamente para $N \rightarrow \infty$ y expandir las funciones de Green y los observables en potencias de $\frac{1}{N}$.

Hay que tener cuidado con la forma de escalear los demás parámetros a medida que $N$ tiende a infinito, para que la teoría siga siendo asintóticamente libre y mantenga constante el valor de $\Lambda_{Q C D}$. Esto se logra manteniendo constante el parámetro $\lambda \equiv g_{Y M}^{2} N$ al tender $N \rightarrow \infty$, de manera que $\lambda \ll 1 \ll N$. Esta convención se conoce como límite de 't Hooft, y es válida no solo para la teoría de YM pura sino que pueden incluirse también fermiones y escalares. En el caso particular de que la teoría de gauge sea conforme, también es posible tomar el límite de $\lambda$ grande, a saber $1 \ll \lambda \ll N$, dando lugar al llamado límite de Maldacena.

Puede demostrarse fácilmente [27] que en el límite de $N$ grande los diagramas no planares en la notación de doble línea están suprimidos por un factor $\frac{1}{N^{2}}$, mientras que los loops de quarks internos lo están por factores $\frac{1}{N}$. Además, para los bilineales de quarks (como $\bar{q} q$ y $\bar{q} \gamma^{\mu} q$ ) los diagramas dominantes tienen quarks solamente en el borde del diagrama. Si bien la suma de todos los diagramas que contribuyen a cada proceso es técnicamente imposible de realizar, pueden extraerse características cualitativas del comportamiento dominante en el límite de $N$ grande.

Si suponemos que QCD, confinante para $N=3$, sigue siendo confinante para $N \rightarrow \infty$, podemos llegar a las siguientes propiedades:

- Los mesones y glueballs son libres, estables y no interactuantes. Las masas mesónicas no divergen y el número de estados mesónicos y de glueballs es infinito; estos están desacoplados entre sí al orden más bajo en $\frac{1}{N}$.

- Las amplitudes de decaimiento para mesones y las de mezcla glueball-mesón son de orden $\frac{1}{\sqrt{N}}$, mientras que las amplitudes de colisión elástica mesón-mesón y las de decaimiento de un glueball en dos mesones o en dos glueballs son de orden $\frac{1}{N}$, y las de colisión elástica glueball-glueball y glueball-mesón de orden $\frac{1}{N^{2}}$. Estas amplitudes elásticas vienen dadas por la suma de diagramas tipo árbol de intercambio de mesones físicos (al igual que en la fenomenología de Regge). Un vértice general con $k$ mesones y $l$ glueballs está dado por $N^{-l-k / 2+1}$ si $k>0$ y $N^{-l+2}$ si $k=0$.

- la regla de Zweig es exacta para $N \rightarrow \infty$ : se encuentran suprimidas las mezclas de singletes y octetes de mesones con gluones, mientras que los mesones se acomodan en nonetes. Los 
mesones en el límite de $N$ grande son estados puros $\bar{q} q$.

Algunos éxitos fenomenológicos del límite de $N$ grande:

- Supresión del mar de $\bar{q} q$ en la física hadrónica (exóticos $\bar{q} q \bar{q} q$ suprimidos).

- Regla de Zweig: los mesones vienen en nonetes de $S U(3)$ de sabor.

- Los decaimientos a varias partículas de mesones inestables están dominados por resonancias de dos partículas.

- Fenomenología de Regge.

- La conexión con teorías de cuerdas.

Todos estos puntos se explican satisfactoriamente con la expansión $\frac{1}{N}$. Hablaremos del último punto en mayor detalle en la próxima Sección.

Para el caso de bariones, el comportamiento no es tan sencillo. Por ejemplo, en los procesos de dispersión que incluyan bariones las amplitudes no están suprimidas para $N \rightarrow \infty$. Puede verse [27] que los bariones, compuestos por $N$ quarks, tienen masa infinita y pueden interpretarse como análogos a los monopolos de Polyakov-'t Hooft, cuya masa diverge como la inversa del acoplamiento.

\subsection{La correspondencia $A d S / C F T$}

La relación entre teorías de gauge y teorías de cuerdas en $A d S$ surgió a partir del estudio de $\mathrm{D} p$-branas y agujeros negros en la teoría de cuerdas. Las $\mathrm{D} p$-branas son solitones de la teoría de cuerdas y su volumen de mundo vive en $p+1$ dimensiones [28]. En la teoría de perturbaciones de cuerdas se definen simplemente a partir de las condiciones de contorno para las cuerdas abiertas fundamentales. Los modos no masivos de estas cuerdas abiertas describen un campo de gauge dentro del volumen de mundo de la $\mathrm{D} p$-brana. En caso de tener $N \mathrm{D} p$-branas coincidentes este campo de gauge es $U(N)$. Las D $p$-branas tienen carga respecto de un potencial de gauge representado por una $(p+1)$-forma y cuyo tensor de campo es una $(p+2)$-forma. Este potencial es parte de los modos no masivos de cuerdas cerradas, que forman parte del multiplete de supergravedad (campos no masivos en la teoría de cuerdas en espacio plano). Al agregar las $\mathrm{D} p$ branas estas generan un flujo del tensor de campo correspondiente, lo que contribuye al tensor de energía-impulso y redunda en una geometría curva. Este caso es similar al de un agujero negro extremalmente cargado en relatividad general, reemplazando al agujero negro por una $p$-brana negra $\left.\right|^{1}$

El caso particular que nos compete en primera instancia es el de $N$ D3-branas coincidentes. En el límite de horizonte cercano (near-horizon), la geometría resultante es $A d S_{5} \times S^{5}$. La teoría correspondiente al límite de bajas energías de la teoría de cuerdas es una teoría de gauge con grupo $U(N)$ y $\mathcal{N}=4$ supersimetrías. Ambas descripciones son perturbativamente válidas en distintos rangos de la constante de acoplamiento: la teoría cuántica de campos perturbativa $(p Q F T)$ es válida cuando la constante de acoplamiento $\lambda=g_{s} N$ es pequeña, mientras que la descripción gravitatoria de bajas energías lo será cuando el radio de curvatura sea mucho mayor que la escala de la cuerda, $R^{2} \gg \alpha^{\prime} \rightarrow \lambda \gg 1$. Desde el lado gravitatorio, al acercar un objeto al horizonte de la brana negra su energía medida por un observador externo se corre al rojo debido al fuerte potencial gravitatorio, y la energía resulta muy pequeña. Por otro lado, las excitaciones

\footnotetext{
${ }^{1}$ Una $p$-brana es un objeto extendido en $p$ dimensiones espaciales, solución de supergravedad.
} 
de baja energía de las branas vienen gobernadas por la teoría de YM. Esto sugiere conjeturar que la teoría de YM para acoplamiento fuerte describe la región de horizonte cercano de la brana negra, cuya geometría es $A d S_{5} \times S^{5}$.

Se ha logrado entender que las cuerdas asociadas a estas teorías de gauge son similares a las cuerdas ordinarias estudiadas desde años antes como teoría unificadora de la física. A diferencia del enfoque tradicional en la teoría de cuerdas, en el presente caso la geometría del espacio es curva $\left(A d S_{5}\right)$ en lugar de plana $\left(\mathbb{M}^{4}\right)$. Igualmente, dado que el número $N$ de D-branas está relacionado con el radio de curvatura $R$ del espacio, el límite de planar coincide con el de curvatura pequeña. El mapeo entre la teoría de gravedad y la de campos es no trivial, dado que estas están definidas en distintas dimensiones de espaciotiempo. Puede pensarse (y de hecho es la imagen habitual) que la teoría de campos está definida en el borde del espaciotiempo de la teoría de gravedad. Esta interpretación es la que da lugar al término de holografía para describir a la correspondencia $A d S / C F T$ [29, 30, 31].

La forma práctica de realizar cálculos de funciones de correlación en $A d S / C F T$ es mediante el Ansatz de Witten [24]. Si $\phi$ es un campo arbitrario definido en el espacio $A d S_{5}$ y $\phi_{0}$ su valor de borde, es decir $\phi\left(x^{\mu}, r\right) \rightarrow \phi\left(x^{\mu}\right)$ para $r \rightarrow \infty$, entonces

$$
\mathcal{Z}\left[\phi_{0}\right]=\lim _{\phi \rightarrow \phi_{0}} e^{-S_{S U G R A}[\phi]},
$$

donde el lado izquierdo de la igualdad es la función generatriz de las funciones de Green conexas de la teoría de campos para $\phi_{0}$ y el lado derecho el límite de la función generatriz de supergravedad para el campo $\phi$. A partir de este Ansatz pueden calcularse las funciones de correlación de $n$ puntos como

$$
\begin{aligned}
\left\langle 0\left|\hat{T}\left\{\hat{O}\left(x_{1}\right) \ldots \hat{O}\left(x_{n}\right)\right\}\right| 0\right\rangle & =\left.\frac{\delta^{(n)} \mathcal{Z}\left[\phi_{0}\right]}{\delta \phi_{0}\left(x_{1}\right) \ldots \delta \phi_{0}\left(x_{n}\right)}\right|_{\phi_{0}=0} \\
& =\frac{\delta^{(n)}}{\delta \phi_{0}\left(x_{1}\right) \ldots \delta \phi_{0}\left(x_{n}\right)} \lim _{\phi \rightarrow \phi_{0}} e^{-S_{\text {SUGRA }}[\phi]} .
\end{aligned}
$$

En la notación de Gubser, Klebanov y Polyakov [25] este Ansatz tiene una expresión algo diferente. Si ahora la funcional generatriz de las funciones de Green conexas de la teoría de gauge para el campo $\phi_{0}$ es $W\left[\phi_{0}\left(x^{\mu}\right)\right] \equiv \mathcal{Z}\left[\phi_{0}\right]$, igualamos esta con el mínimo $K$ de la acción de supergravedad $S$, con condiciones de contorno fijas en $r \rightarrow \infty$,

$$
W\left[\phi_{0}\left(x^{\mu}\right)\right]=K\left[\phi_{0}\left(x^{\mu}\right)\right]
$$

con

$$
\left.K\left[\phi_{0}\left(x^{\mu}\right)\right] \equiv \min S\left[\phi\left(x^{\mu} . r\right)\right]\right|_{b c} .
$$

Para que esta expresión sea equivalente a (2.2.1) debe evaluarse la acción de supergravedad $S$ término a término en su desarrollo en el campo $\phi$.

Puede verse una introducción más detallada a la correspondencia $A d S / C F T$ en [32]. 


\section{Capítulo 3}

\section{Amplitudes de dispersión en teoría de cuerdas: construcción de lagrangianos efectivos}

\subsection{Método para calcular lagrangianos efectivos en la teoría de cuerdas}

Si quisiéramos describir la teoría de cuerdas en toda su extensión, deberíamos lidiar con objetos extendidos, cuyo espectro contiene un número infinito de fluctuaciones que interpretamos como partículas. Si quisiéramos describirlo en términos de campos locales deberíamos introducir un número infinito de ellos. Podemos quedarnos solamente con un número finito de ellos, los no masivos, y con esto definir una teoría para las fluctuaciones de bajas energías. Esto introduce la no localidad.

En esta Sección derivaremos, basados en [33], un enfoque basado en lagrangianos efectivos para estos campos bosónicos no masivos. Consideraremos los campos fermiónicos apagados. Estos desarrollos valen en la forma aquí presentada para teorías de supercuerdas del tipo II definidas sobre espacio plano $\mathbb{M}_{10}$. Estudiaremos al final de esta Sección cómo convertirlas en amplitudes en espacio curvo bajo ciertas condiciones. Utilizaremos el enfoque de la matriz $S$ para calcular la acción efectiva orden a orden en el número de partículas interactuantes. Calcularemos explícitamente términos de lagrangianos efectivos de 2, 3 y 4 campos, que son los que utilizaremos en el Capítulo 7, en la Segunda Parte.

La forma más completa de llegar a las ecuaciones de movimiento (en adelante EOM, del inglés Equations Of Motion) para las cuerdas es con la teoría de campos de cuerdas (String Field Theory [34, 35]) . Las ecuaciones que salen de esta, sin embargo, son ecuaciones diferenciales funcionales. Este campo funcional puede reemplazarse por un número infinito de campos locales, uno por cada partícula descripta por la cuerda. La ecuación para la funcional puede reemplazarse por un número infinito de ecuaciones diferenciales ordinarias para estos campos. Si expandimos ahora en momentos para $\left|p^{2}\right| \ll M_{\text {Planck }}^{2}$, estas resultan en un número finito de ecuaciones diferenciales ordinarias. Estas ecuaciones son no locales pero, a cualquier orden finito, son ecuaciones usuales en derivadas parciales que pueden pensarse provenientes de un lagrangiano efectivo ordinario.

Hay dos métodos indirectos para conseguir este lagrangiano efectivo sin tener que realizar el desarrollo en momentos partiendo desde la teoría de cuerdas. El primero consiste en construir la teoría de cuerdas con campos de fondo (background fields). Las condiciones de consistencia para la teoría de cuerdas resultan en EOMs para estos campos. Si bien este método hace explícitas 
las simetrías en diez dimensiones y pueden obtenerse resultados válidos a todo orden de perturbaciones, requiere el cálculo a $n$ loops de la función $\beta$ para derivar el $n$-ésimo término de la acción efectiva. Este método fue desarrollado por varios grupos [36, 37, 38, 39, 40, 41].

El otro método, que es el que utilizaremos en el presente trabajo, fue desarrollado en [42, 43, 44, 45] y es más sencillo. Consiste en calcular simplemente la amplitud de un dado proceso de dispersión de partículas no masivas a nivel árbol desde teoría de cuerdas y luego definir un lagrangiano efectivo que resproduzca esta misma amplitud. Esto puede hacerse de manera perturbativa: primero se construye $\mathcal{L}_{2 p t}$ que describe las partículas no masivas libres. Luego agregamos términos cúbicos para describir los acoplamientos de tres partículas dados por el vértice de cuerdas, obteniendo $\mathcal{L}_{3 p t}$. En tercer lugar consideramos las amplitudes de cuatro puntos. Por unitariedad, los polos no masivos serán simplemente los generados por los diagramas árbol de $\mathcal{L}_{3 p t}$. El resto se debe al intercambio de partículas masivas: no tiene singularidades para momentos externos pequeños y puede por ende expandirse en potencias de $p^{2}$. Cada término en esta expresión puede reproducirse por un vértice local $V_{4 p t}$ y de aquí construimos el $\mathcal{L}_{4 p t}$. Puede repetirse este procedimiento para términos de interacción de más puntos y de esta manera conseguir un $\mathcal{L}_{\text {eff }}$ a todo orden. Este proceso puede simplificarse teniendo en cuenta las simetrías locales y globales de la teoría. El $\mathcal{L}_{\text {eff }}$ así calculado no será único sino que pueden redefinirse los campos de manera que las amplitudes permanezcan invariantes.

\section{Lagrangiano de 2 puntos}

Ilustremos este método calculando la acción efectiva de bajas energías para supercuerdas cerradas tipo II (tanto IIA como IIB). Solo consideramos bosones no masivos en el sector de supergravedad del tipo $\mathcal{N}=1$, a saber el gravitón $h_{a b}$, el tensor antisimétrico $B_{a b}$ y el dilatón $\Phi$, presentes tanto en supergravedad IIA como IIB (en adelante, SUGRA IIA y IIB respectivamente). Los términos cuadráticos en la acción implican una acción efectiva $S_{2 p t}^{\text {eff }}=\int d^{10} x \sqrt{-g} \mathcal{L}_{2 p t}$ con $g$ la métrica del fondo $\mathrm{y}$

$$
\mathcal{L}_{2 p t}=\frac{1}{2 \kappa^{2}} R-\frac{1}{6} e^{-2 c \Phi} H_{a b c} H^{a b c}-\frac{1}{2}\left(\nabla_{a} \Phi\right)\left(\nabla^{a} \Phi\right),
$$

donde

$$
H_{a b c}=\partial_{[c} B_{a b]}=\partial_{c} B_{a b}+\partial_{a} B_{b c}+\partial_{b} B_{c a},
$$

y utilizamos $\kappa=\frac{1}{\sqrt{8 \pi G_{10}}}$ y $c$ como constantes de acoplamiento.

\section{Lagrangiano de 3 puntos}

Queremos ahora $\mathcal{L}_{3 p t}$, para lo que necesitamos las amplitudes de 3 puntos. Sabemos que la amplitud de cuerdas cerradas para la dispersión de $N$ partículas puede escribirse como una suma de productos de amplitudes de cuerdas abiertas moviéndose hacia la derecha y hacia la izquierda. Para el caso de amplitudes de 3 y 4 puntos solo hay un término en esta suma

$$
\begin{aligned}
A_{\text {closed }}(1,2,3) & =4 g_{s} f(1,2,3) \tilde{A}_{\text {right }}(1,2,3) \otimes A_{\text {left }}(1,2,3), \\
A_{\text {closed }}(1,2,3,4) & =2 g_{s}^{2} f(1,2,3,4) \tilde{A}_{\text {right }}(1,2,3,4) \otimes A_{\text {left }}(1,2,3,4),
\end{aligned}
$$

donde $g_{s}$ es la constante de acoplamiento de la cuerda. Notando $k_{i}$ el momento y $\rho_{i}$ el vector polarización de la $i$-ésima partícula, la amplitud de 3 puntos es [46]

$$
A^{\text {open }}\left(k_{1}, k_{2}, k_{3}, \rho_{1}, \rho_{2}, \rho_{3}\right)=\rho_{1} \cdot k_{2} \rho_{2} \cdot \rho_{3}+\rho_{2} \cdot k_{3} \rho_{3} \cdot \rho_{1}+\rho_{3} \cdot k_{1} \rho_{1} \cdot \rho_{2} .
$$


Podemos definir un tensor de rango 2 que denote la polarización de las cuerdas cerradas que se factorizará como $\Theta_{m n}=\rho_{m} \otimes \rho_{n}$, con lo que obtenemos

$$
\begin{aligned}
A_{3 p t}= & 4 g \frac{1}{2}\left[\tilde{\rho_{1}} \cdot k_{2} \tilde{\rho_{2}} \cdot \tilde{\rho_{3}}+\tilde{\rho_{2}} \cdot k_{3} \tilde{\rho_{3}} \cdot \tilde{\rho_{1}}+\tilde{\rho_{3}} \cdot k_{1} \tilde{\rho_{1}} \cdot \tilde{\rho_{2}}\right] \otimes \\
& \frac{1}{2}\left[\rho_{1} \cdot k_{2} \rho_{2} \cdot \rho_{3}+\rho_{2} \cdot k_{3} \rho_{3} \cdot \rho_{1}+\rho_{3} \cdot k_{1} \rho_{1} \cdot \rho_{2}\right] \\
= & g\left[\left(k_{2} \Theta_{1} k_{2}\right) \operatorname{Tr}\left(\Theta_{1} \Theta_{3}^{t}\right)+\left(k_{3} \Theta_{2} \Theta_{3}^{t} \Theta_{1} k_{2}\right)+\left(k_{1} \Theta_{3} \Theta_{2}^{t} \Theta_{1} k_{2}\right)\right]+\text { permutaciones cíclicas, }
\end{aligned}
$$

donde el supraíndice $t$ denota transposición de matrices. El momento de cada amplitud parcial de cuerdas abiertas debe tomarse $\frac{k_{i}}{2}$, donde $k_{i}$ es el momento de la cuerda cerrada correspondiente. Utilizamos aquí la notación matricial, en la que por ejemplo $k_{2} \Theta_{1} k_{2} \equiv k_{2}^{m} \Theta_{m n}^{1} k_{2}^{n}$. Para encontrar las amplitudes de tres puntos posibles de construir basta con tomar cada $\Theta_{m n}$ como uno de los campos representados por cuerdas cerradas $\left(h_{a b}, B_{a b}\right.$ y $\left.\Phi\right)$, según la colisión que se desee estudiar. Estas polarizaciones son, respectivamente

$$
\begin{aligned}
\Theta_{m n} & \rightarrow h_{m n}, \\
\Theta_{m n} & \rightarrow B_{m n} \\
\Theta_{m n} & \rightarrow \sqrt{\frac{1}{8}}\left(\eta_{m n}-k_{m} \bar{k}_{n}-\bar{k}_{m} k_{n}\right) \phi
\end{aligned}
$$

donde introdujimos los vectores $\bar{k}_{i}$ que cumplen las condiciones $\bar{k} \cdot \bar{k}=0$ y $k \cdot \bar{k}=1$. Estos corresponden a estados longitudinalmente polarizados que se desacoplarán del proceso físic ${ }^{1}$, y son introducidos para satisfacer la condición de transversalidad $k^{m} \Theta_{m n}=0$. Hemos utilizado la métrica $\eta_{m n}$ correspondiente al espacio plano de diez dimensiones $\mathbb{M}_{10}$.

Veremos ahora un ejemplo de cómo funciona esto calculando los términos del lagrangiano efectivo correspondientes a las amplitudes para los procesos hhh y $B B \Phi$. La primera es

$$
A_{h h h}=g_{s}\left[\left(k_{2} h_{1} k_{2}\right) \operatorname{Tr}\left(h_{1} h_{3}^{t}\right)+\left(k_{3} h_{2} h_{3}^{t} h_{1} k_{2}\right)+\left(k_{1} h_{3} h_{2}^{t} h_{1} k_{2}\right)\right]+\text { permutaciones cíclicas }
$$

y puede verse que corresponde al término del lagrangiano

$$
\mathcal{L}_{h h h}=-\frac{1}{2} g_{s}\left(h^{a b} h^{c d} \partial_{a} \partial_{b} h_{c d}+2 \partial^{d} h_{a b} \partial^{a} h^{b c} h_{c d}\right)
$$

donde identificamos $k_{i} \rightarrow i \partial_{i}$. Este término ya estaba incluido en el lagrangiano $\mathcal{L}_{2 p t}$, pues

$$
\left.\frac{1}{\kappa^{2}} \sqrt{-g} R\right|_{3 p t}=-\kappa\left(h^{a b} h^{c d} \partial_{a} \partial_{b} h_{c d}+2 \partial^{d} h_{a b} \partial^{a} h^{b c} h_{c d}\right)
$$

con lo que vemos que no necesitamos agregar un nuevo término para reproducir esta amplitud, y además demostramos que $2 \kappa=g_{s}\left(2 \alpha^{\prime}\right)^{2}$ es la forma correcta de relacionar las constantes de acoplamiento $2^{2}$

Para el caso del proceso $B B \Phi$, resulta

$$
A_{B B \Phi}=-g_{s} \sqrt{2} \Phi_{3}\left(k_{2} B_{1} B_{2} k_{1}\right)
$$

\footnotetext{
${ }^{1}$ Ver [46], página 191

${ }^{2}$ Agregamos aquí el factor $\alpha^{\prime}$, pues estamos utilizando la convención $\alpha^{\prime}=\frac{1}{2}$ en las amplitudes de dispersión provenientes de la teoría de cuerdas.
} 
$\mathrm{Si}$ a esta amplitud le sumamos un término de la forma $\frac{g_{s}}{2 \sqrt{2}} \phi \partial_{c} B_{a b} \partial^{c} B^{a b}=\frac{\kappa}{\sqrt{2}} \partial_{c} B_{a b} \partial^{c} B^{a b}$, pues $k_{I} \cdot k_{J}=0$ para tres partículas físicas no masivas, obtenemos

$$
\kappa \sqrt{2} \phi\left[\partial_{c} B_{a b} \partial^{a} B^{b c}+\frac{1}{2} \partial_{c} B_{a b} \partial^{c} B^{a b}\right]
$$

De la definición (3.1.2) vemos que resulta

$$
\mathcal{L}=\frac{1}{6} \sqrt{2} \kappa \phi H_{a b c} H^{a b c}
$$

que es el segundo término en la expansión de

$$
\mathcal{L}=-\frac{1}{6} e^{-\sqrt{2} \kappa \phi} H_{a b c} H^{a b c}
$$

Este término también aparecía ya en $\mathcal{L}_{2 p t}$, por lo que no necesitamos agregar términos nuevos y extraemos la relación $c=\frac{1}{\sqrt{2}} \kappa$ entre las constantes de acoplamiento.

\section{Lagrangiano de 4 puntos}

En el caso de un lagrangiano de 4 puntos de cuerdas abiertas, la amplitud toma la forma

$$
A^{\text {open }}\left(k_{1}, k_{2}, k_{3}, k_{4}, \rho_{1}, \rho_{2}, \rho_{3}, \rho_{4}\right)=-\frac{1}{2} g_{s}^{2} \frac{\Gamma(-s / 2) \Gamma(-t / 2)}{\Gamma(1+u / 2)} K(1,2,3,4)
$$

donde $s=-\left(k_{1}+k_{2}\right)^{2}, t=-\left(k_{1}+k_{4}\right)^{2}$ y $u=-\left(k_{1}+k_{3}\right)^{2}$ son las variables de Mandelstam y $K$ es el factor cinemático

$$
\begin{array}{rll}
K=- & \frac{1}{4} & \left(s t \rho_{1} \cdot \rho_{3} \rho_{2} \cdot \rho_{4}+s u \rho_{2} \cdot \rho_{3} \rho_{1} \cdot \rho_{4}+t u \rho_{1} \cdot \rho_{2} \rho_{3} \cdot \rho_{4}\right)+ \\
\frac{1}{2} & s\left(\rho_{1} \cdot k_{4} \rho_{3} \cdot k_{2} \rho_{2} \cdot \rho_{4}+\rho_{2} \cdot k_{3} \rho_{4} \cdot k_{1} \rho_{1} \cdot \rho_{3}\right. \\
& + & \left.\rho_{1} \cdot k_{3} \rho_{4} \cdot k_{2} \rho_{2} \cdot \rho_{3}+\rho_{2} \cdot k_{4} \rho_{3} \cdot k_{1} \rho_{1} \cdot \rho_{4}\right) \\
& \frac{1}{2} & t\left(\rho_{2} \cdot k_{1} \rho_{4} \cdot k_{3} \rho_{3} \cdot \rho_{1}+\rho_{3} \cdot k_{4} \rho_{1} \cdot k_{2} \rho_{2} \cdot \rho_{4}\right. \\
& + & \left.\rho_{2} \cdot k_{4} \rho_{1} \cdot k_{3} \rho_{3} \cdot \rho_{4}+\rho_{3} \cdot k_{1} \rho_{4} \cdot k_{2} \rho_{2} \cdot \rho_{1}\right) \\
& \frac{1}{2} & u\left(\rho_{1} \cdot k_{2} \rho_{4} \cdot k_{3} \rho_{3} \cdot \rho_{2}+\rho_{3} \cdot k_{4} \rho_{2} \cdot k_{1} \rho_{1} \cdot \rho_{4}\right. \\
& + & \left.\rho_{1} \cdot k_{4} \rho_{2} \cdot k_{3} \rho_{3} \cdot \rho_{4}+\rho_{3} \cdot k_{2} \rho_{4} \cdot k_{1} \rho_{1} \cdot \rho_{2}\right)
\end{array}
$$

Para la amplitud de cuerdas cerradas tenemos

$$
\begin{aligned}
A_{4}^{\text {closed }} & =-i \pi^{2} g_{s}^{2} \alpha^{\prime 3} \frac{\Gamma\left(-\frac{\alpha^{\prime} s}{4}\right) \Gamma\left(-\frac{\alpha^{\prime} u}{4}\right) \Gamma\left(-\frac{\alpha^{\prime} t}{4}\right)}{\Gamma\left(1+\frac{\alpha^{\prime} s}{4}\right) \Gamma\left(1+\frac{\alpha^{\prime} u}{4}\right) \Gamma\left(1+\frac{\alpha^{\prime} t}{4}\right)} \times K_{4}^{\text {closed }} \\
& =-i \pi^{2} g_{s}^{2} \alpha^{\prime 3} \frac{\Gamma\left(-\frac{\alpha^{\prime} s}{4}\right) \Gamma\left(-\frac{\alpha^{\prime} u}{4}\right) \Gamma\left(-\frac{\alpha^{\prime} t}{4}\right)}{\Gamma\left(1+\frac{\alpha^{\prime} s}{4}\right) \Gamma\left(1+\frac{\alpha^{\prime} u}{4}\right) \Gamma\left(1+\frac{\alpha^{\prime} t}{4}\right)} \times \tilde{K}_{4}^{\text {open }} \otimes K_{4}^{\text {open }}
\end{aligned}
$$

donde $K_{\text {left }}$ y $K_{\text {right }}$ vienen dados por 3.1.18. Desarrollaremos un cálculo explícito de este tipo en la Sección 7.1.1.

\subsection{Amplitudes de dispersión en espacio curvo}

Hemos deducido hasta aquí un método para construir lagrangianos efectivos que dan resultados equivalentes a los dados por la teoría de cuerdas para el límite de bajas energías (modos 
no masivos) que es válido para espacio plano. Queremos ahora estudiar la validez de este método para el caso de espacio curvo, en particular para espacios de la forma $A d S_{5} \times W$, donde $W$ es un espacio de Einstein compacto. Estamos interesados en la región $N \gg \lambda \gg 1$, lo que simplificará enormemente los resultados. Como estamos en el límite de $N$ grande, solamente nos interesaremos por las hojas de mundo topológicamente esféricas (i.e. de genus $g=0$ ). Dado que $\lambda=\frac{R^{4}}{\alpha^{\prime 2}}$, el límite de $\lambda$ grande implica $\alpha^{\prime} \ll R^{2}$, lo que quiere decir que el radio de curvatura del $A d S$ es mucho mayor que el tamaño de las cuerdas. Esto nos indica que el acoplamiento de la cuerda sobre la hoja de mundo $\frac{1}{\sqrt{\lambda}}$ es pequeño, con lo que la integral de camino en la hoja de mundo es prácticamente gaussiana. Podemos entonces separar los campos definidos en la hoja de mundo de la cuerda en sus modos cero y sus modos excitados en la forma [47]

$$
X^{M}(\tau, \sigma)=x^{M}+\tilde{X}^{M}(\tau, \sigma) .
$$

Para un dado $x^{M}$ fijo, la integral gaussiana sobre los modos excitados es exactamente la misma que tendríamos en espaciotiempo pland? lo que produce una matriz $S$ localmente equivalente al caso de espacio plano diez-dimensional con una amplitud $i A_{\text {local }}\left(x^{M}\right)$. Integrando el modo cero obtenemos una matriz $S$ de la forma

$$
S=i \int d^{10} x \sqrt{-g} A_{\text {local }}\left(x^{M}\right)
$$

Definimos $A_{\text {local }}$ de manera que sea un escalar, y $\sqrt{-g}$ viene de la medida de la integral de camino. Esta aproximación local pierde validez en el caso de radios de curvatura comparables con la longitud de la cuerda fundamental, $R^{2} \sim \alpha^{\prime}$, lo que será relevante cuando estudiemos nuestro problema para valores del parámetro de Bjorken $x$ exponencialmente pequeño en el Capítulo 7 [47].

\footnotetext{
${ }^{3}$ Estamos despreciando los modos de Ramond-Ramond del fondo, pues las perturbaciones que inducen son subdominantes en este régimen con curvatura pequeña $\frac{R^{2}}{\alpha^{\prime}}=\sqrt{\lambda} \gg 1$.
} 


\section{Capítulo 4}

\section{Dispersión inelástica profunda (DIS)}

La forma usual de estudiar la estructura de la materia a nivel de partículas elementales es mediante experimentos de colisión. La energía de las partículas en colisión nos dará la escala a la que estamos estudiando el sistema, correspondiendo las mayores energías a los tamaños más pequeños. De esta forma se descubrió la estructura interna del átomo, compuesta por un núcleo y una nube de electrones, haciendo colisionar partículas $\alpha$ contra láminas de oro (experimento de Rutherford). Más tarde se estudió la estructura del núcleo haciendo colisionar electrone $\$^{1}$ sobre este, descubriendo su composición en nucleones (protones y neutrones). También se descubrieron muchos otros bariones (fermiones) y mesones (bosones), partículas llamadas genéricamente hadrones.

Para estudiar la estructura interna de los hadrones se comenzaron a realizar experimentos de colisión de hadrones con electrones muy energéticos, mostrando una subestructura para los protones [48]. La fuerza electromagnética entre el electrón y el hadrón es mediada por un fotón virtual, lo que es descripto por la electrodinámica cuántica (QED, del inglés Quantum ElectroDynamics). En la colisión con electrones de altas energías se intercambia este fotón virtual con un gran cuadrimomento $q^{2}$ entre el electrón y el hadrón, lo que permite estudiar la estructura interna del último. La colisión en este régimen es lo que denominamos dispersión inelástica profunda (DIS, del inglés Deep Inelastic Scattering) y es la que permitió el desarrollo del modelo de partones (quarks y gluones), que son los constituyentes del protón. La sección eficaz para un proceso de DIS puede expresarse en términos de un número de factores cinemáticos denominados funciones de estructura del hadrón. La determinación de estas funciones de estructura a partir de las secciones eficaces da información de las funciones de distribución partónicas (PDF, del inglés Parton Distribution Functions) y por ende de la interacción entre los quarks y gluones dentro del protón. Se encontró que, como se predice para protones compuestos de constituyentes puntuales, en el rango cinemático del DIS las funciones de estructura son prácticamente independientes del momento intercambiado $q^{2}$. Esta propiedad se denomina escaleo (scaling). Al estudiar este proceso a un orden más de aproximación, teniendo en cuenta efectos de cromodinámica cuántica en el régimen perturbativo (pQCD), se observó que las funciones de estructura no mostraban más un escaleo sino que tenían una dependencia logarítmica con $q^{2}$.

Estudiaremos en esta Sección la interacción entre un fotón y un hadrón arbitrario (de espín $0, \frac{1}{2}$ o 1 ) en el régimen de DIS. En este régimen cinemático, en el que el momento transferido

\footnotetext{
${ }^{1}$ Hablaremos durante esta Tesis indistintamente de electrones o leptones cargados, pero los resultados se corresponden con igual validez para muones. La dispersión de leptones $\tau$, si bien abarcada en principio por nuestros modelos, es técnicamente imposible en la práctica debido a la corta vida media de estas partículas. Si bien con estos modelos también es posible estudiar DIS con neutrinos, en esta Tesis nos restringiremos exclusivamente a leptones cargados. Con las consideraciones apropiadas, pueden hacerse estudios de DIS con neutrinos dentro del marco de los modelos holográficos citados.
} 


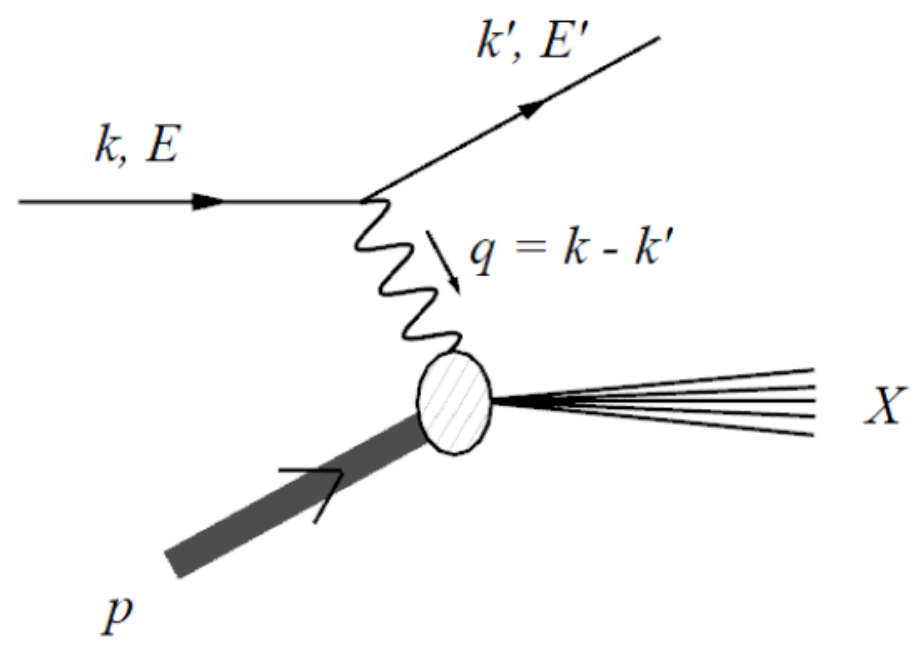

Figura 4.1: Diagrama esquemático de la dispersión inelástica profunda leptón-hadrón. El momento del hadrón incidente es $P$ y el del fotón virtual es $q$. El estado hadrónico final que no se mide- es $X$. Figura tomada de [50].

$q^{2}$ es mucho mayor que la escala de confinamiento de la teoría, el hadrón se fragmenta en varias partículas o bien se convierte en un estado hadrónico excitado. Al primer orden este proceso puede describirse como la dispersión electromagnética del leptón por un quark dentro del hadrón [49]. Esta dispersión produce gluones y pares quark-antiquark que finalmente hadronizan emitiendo un haz en la dirección del momento transferido. Típicamente la masa del sistema hadrónico final es grande comparada con la masa del hadrón incidente. Las propiedades del DIS que estudiaremos en este Capítulo son aquellas que pueden ser derivadas de QCD usando la teoría cuántica de campos, sin recurrir a modelos fenomenológicos efectivos. Utilizaremos la notación de Manohar [50], con la diferencia de que definiremos aquí la métrica de Minkowski como $\eta_{\mu \nu} \equiv$ $\operatorname{diag}(-1,+1,+1,+1)$, es decir, con la signatura opuesta a la de la referencia mencionada.

\subsection{Cinemática del proceso}

En un proceso de DIS se dispersa un haz incidente de leptones con energía $E$ y momento $\vec{k}$ por un blanco hadrónico fijo. Puede medirse la energía del leptón dispersado, así como su energía. El leptón interactúa con el hadrón por medio del intercambio de un fotón virtual de momento $q^{\mu}$. El hadrón absorbe el fotón, produciendo un estado final $X$, que no puede ser medido del experimento, por lo que se denomina a este un proceso inclusivo. Con este proceso, se estudia la estructura del hadrón en tamaños del orden de $\frac{1}{q}$, con $q \equiv \sqrt{q^{2}}$. Si el hadrón permanece intacto, el proceso es una dispersión elástica, mientras que si se fragmenta es un proceso de DIS. En la Figura 4.1 podemos ver un esquema de este proceso.

Las variables cinemáticas son:

- $M$ es la masa del blanco hadrónico, con la condición de capa de masa $M^{2}=-P^{2}$.

- E es la energía del leptón incidente.

- $k^{\mu}=(E, 0,0, E)$ es el momento del leptón incidente 2

\footnotetext{
${ }^{2}$ Estamos en la aproximación en que despreciamos la masa del leptón frente a su energía, $E \gg m_{l}$.
} 
- $k^{\prime \mu}=\left(E^{\prime}, E^{\prime} \sin \theta \cos \phi, E^{\prime} \sin \theta \sin \phi, E^{\prime} \cos \phi\right)$ es el momento del leptón dispersado ${ }^{3}$

- $E^{\prime}$ es la energía del leptón dispersado.

- $P^{\mu}=(M, 0,0,0)$ es el momento del hadrón blanco. ${ }^{4}$

- $q^{\mu}=k^{\mu}-k^{\prime \mu}$, es el momento del fotón virtual, transferido durante el proceso.

- $\nu=E-E^{\prime}=-\frac{P \cdot q}{M}$ es la pérdida de energía del leptón.

- $y=\frac{\nu}{E}=\frac{P \cdot q}{P \cdot k}$ es la pérdida de energía fraccional del leptón.

- $x=-\frac{q^{2}}{2 P \cdot q}$ es el parámetro de Bjorken.

- $t=\frac{P^{2}}{q^{2}}$ es un parámetro que en nuestro régimen cumplirá $|t| \ll 1$.

Definidos estos parámetros, podemos redefinir la dispersión inelástica profunda como la dispersión de un leptón por un hadrón en el límite en que $x$ se mantiene fijo y $q \gg M, \Lambda_{Q C D}$.

La masa del estado hadrónico final $X$ es:

$$
M_{X}^{2}=-(P+q)^{2}=M^{2}-2 P \cdot q-q^{2} .
$$

de donde puede verse que

$$
x=-\frac{q^{2}}{2 P \cdot q}=1+\frac{M_{X}^{2}-M^{2}}{2 P \cdot q} .
$$

por lo que $0 \leq x \leq 1$, donde $x=1$ implica $M_{X}^{2}=M^{2}$ y corresponde a una dispersión elástica.

\subsection{Sección eficaz de dispersión}

La amplitud de dispersión $\mathcal{M}$ para un proceso de dispersión inelástica profunda está dada por:

$$
i \mathcal{M}=(-i e)^{2}\left(\frac{-i \eta_{\mu \nu}}{q^{2}}\right)\left\langle k^{\prime}\left|j_{l}^{\mu}(0)\right| k, s_{l}\right\rangle\left\langle X\left|j_{h}^{\nu}(0)\right| P, h\right\rangle .
$$

donde $e$ es la carga eléctrica del leptón, $s_{l}$ es la polarización del leptón inicial, $h$ es la polarización del hadrón inicial y $j_{l}^{\mu}, j_{h}^{\nu}$ son las corrientes electromagnéticas del leptón y del hadrón, respectivamente. La polarización del hadrón inicial puede elegirse como el espín en la dirección de un eje arbitrario, para lo cual suele utilizarse la dirección del haz incidente.

La sección eficaz diferencial de dispersión se muestra en (4.2.2). Las polarizaciones del leptón final y de los estados hadrónicos finales no se miden, por lo que debe sumarse sobre las mismas, obteniendo

$$
\begin{aligned}
d \sigma= & \sum_{X} \int \frac{d^{3} k^{\prime}}{(2 \pi)^{3} 2 E^{\prime}}(2 \pi)^{4} \delta^{4}\left(k+P-k^{\prime}-P_{X}\right) \frac{|\mathcal{M}|^{2}}{(2 E)(2 M)\left(v_{r e l}=1\right)} \\
= & \sum_{X} \int \frac{d^{3} k^{\prime}}{(2 \pi)^{3} 2 E^{\prime}} \frac{(2 \pi)^{4} \delta^{4}\left(k+P-k^{\prime}-P_{X}\right)}{(2 E)(2 M)} \frac{e^{4}}{q^{4}} \\
& \times\left\langle P, h\left|j_{h}^{\mu}(0)\right| X\right\rangle\left\langle X\left|j_{h}^{\nu}(0)\right| P, h\right\rangle\left\langle k, s_{l}\left|j_{l \mu}(0)\right| k^{\prime}\right\rangle\left\langle k^{\prime}\left|j_{l \nu}(0)\right| k, s_{l}\right\rangle,
\end{aligned}
$$

\footnotetext{
${ }^{3}$ Las variables $\theta$ y $\phi$ son el ángulo polar y el acimutal de las coordenadas esféricas usuales, respectivamente.

${ }^{4}$ Consideramos el sistema de referencia en el que este blanco está en reposo.
} 
donde se usa la relación $\left\langle\alpha\left|j^{\mu}\right| \beta\right\rangle^{*}=\left\langle\beta\left|j^{\mu}\right| \alpha\right\rangle$, lo cual es correcto debido a que la corriente es hermítica, $j_{\mu}^{\dagger}=j_{\mu}$.

Definimos el tensor leptónico $l^{\mu \nu}$ como:

$$
l^{\mu \nu}=\sum_{\text {espín final }}\left\langle k^{\prime}\left|j_{l}^{\nu}(0)\right| k, s_{l}\right\rangle\left\langle k, s_{l}\left|j_{l}^{\mu}(0)\right| k^{\prime}\right\rangle
$$

de manera que los elementos de matriz de la corriente leptónica en 4.2 .2 pueden reemplazarse por $l^{\mu \nu}$.

Definimos el tensor hadrónico $W_{\mu \nu}$ como:

$$
W_{\mu \nu}(P, q)_{h^{\prime} h}=\int d^{4} x e^{i q \cdot x}\left\langle P, h^{\prime}\left|\left[j_{\mu}(x), j_{\nu}(0)\right]\right| P, h\right\rangle,
$$

donde $h$ y $h^{\prime}$ son las polarizaciones del hadrón inicial y final respectivamente. Insertando una base completa de autoestados y utilizando la invariancia ante traslaciones se obtiene

$$
\begin{array}{r}
W_{\mu \nu}(P, q)_{h^{\prime} h}=\frac{1}{4 \pi} \sum_{X}\left[(2 \pi)^{4} \delta^{4}\left(q+P-P_{X}\right)\left\langle P, h^{\prime}\left|j_{\mu}(0)\right| X\right\rangle\left\langle X\left|j_{\nu}(0)\right| P, h\right\rangle\right. \\
\left.-(2 \pi)^{4} \delta^{4}\left(q-P+P_{X}\right)\left\langle P, h^{\prime}\left|j_{\nu}(0)\right| X\right\rangle\left\langle X\left|j_{\mu}(0)\right| P, h\right\rangle\right] .
\end{array}
$$

Los estados finales permitidos deben tener $P_{X}^{0} \geq P^{0}$, ya que $M_{X}^{2} \geq M^{2}$. Como $q^{0}>0,4.2 .5$ se reduce a la expresión (4.2.2) que incluye la corriente hadrónica y la función delta para la conservación del cuadrimomento. Sustituyendo con $l^{\mu \nu}$ y $W_{\mu \nu}$ en 4.2 .2 se obtiene

$$
\begin{gathered}
d \sigma=\frac{e^{4}}{q^{4}} \int \frac{d^{3} k^{\prime}}{(2 \pi)^{3} 2 E^{\prime}} \frac{l^{\mu \nu} W_{\mu \nu}\left(P, k-k^{\prime}\right)_{h h}}{(2 E)(2 M)\left(v_{r e l}=1\right)}, \\
\frac{d^{2} \sigma}{d E^{\prime} d \Omega}=\frac{e^{4}}{64 \pi^{3} q^{4}} \frac{E^{\prime}}{M E} l^{\mu \nu} W_{\mu \nu}(P, q)_{h h} .
\end{gathered}
$$

Toda la información sobre la sección eficaz de la dispersión inelástica profunda está contenida en los tensores leptónico $l^{\mu \nu}$ y hadrónico $W_{\mu \nu}$.

\subsubsection{Tensor leptónico $l^{\mu \nu}$}

El tensor leptónico se calcula a partir de la siguiente ecuación:

$$
l^{\mu \nu}=\sum_{\text {espín final }} \bar{u}\left(k^{\prime}\right) \gamma^{\nu} u\left(k, s_{l}\right) \bar{u}\left(k, s_{l}\right) \gamma^{\mu} u\left(k^{\prime}\right) .
$$

donde $u(k)$ es el espinor de Dirac correspondiente al leptón. Para realizar la suma sobre los estados finales, se usa la identidad

$$
\sum_{\text {espín final }} u\left(k^{\prime}\right) \bar{u}\left(k^{\prime}\right)=-\gamma^{\rho} k_{\rho}^{\prime}-m_{l} .
$$

Para una partícula de espín $1 / 2$ puede definirse el cuadrivector polarización $s_{l}^{\mu}$ como:

$$
2 s_{l}^{\mu}=\bar{u}\left(k, s_{l}\right) \gamma^{\mu} \gamma_{5} u\left(k, s_{l}\right)
$$


El producto de los espinores del estado inicial puede escribirse como

$$
u\left(k, s_{l}\right) \bar{u}\left(k, s_{l}\right)=\left(-\gamma^{\rho} k_{\rho}+m\right) \frac{1-\gamma_{5} \gamma_{\alpha} s_{l}^{\alpha} / m_{l}}{2} .
$$

Finalmente, sustituyendo las ecuaciones (4.2.9) y (4.2.11) en (4.2.8) se obtiene:

$$
\begin{aligned}
l^{\mu \nu} & =\operatorname{Tr}\left[\left(-\gamma^{\rho} k_{\rho}^{\prime}+m_{l}\right) \gamma^{\nu}\left(-\gamma^{\sigma} k_{\sigma}+m_{l}\right) \frac{1-\gamma_{5} \gamma_{\alpha} s_{l}^{\alpha} / m_{l}}{2} \gamma^{\mu}\right], \\
& =2\left[k^{\mu} k^{\prime \nu}+k^{\nu} k^{\prime \mu}-\eta^{\mu \nu}\left(k . k^{\prime}-m_{l}^{2}\right)-i \epsilon^{\mu \nu \alpha \beta} q_{\alpha} s_{l \beta}\right] .
\end{aligned}
$$

Notemos que la parte del tensor leptónico que es independiente del espín, es simétrica frente al intercambio $\mu \leftrightarrow \nu$, y la parte que es dependiente del espín es antisimétrica. En consecuencia, un haz de leptones no polarizado sólo prueba la parte simétrica de $W_{\mu \nu}$.

\subsubsection{Tensor hadrónico $W_{\mu \nu}$}

La estructura del hadrón que es relevante para la dispersión inelástica profunda puede caracterizarse completamente por el tensor hadrónico $W_{\mu \nu}$. A diferencia del tensor leptónico, $W_{\mu \nu}$ no puede calcularse directamente desde QCD debido a los efectos no perturbativos de las interacciones fuertes.

Como fue mostrado por Bjorken [51, 52, 53, 54], si los hadrones están constituidos esencialmente por partones libres de masa nula, los cuales aparecen en la función de onda hadrónica con una dada distribución de momento y energía, entonces esa distribución se puede medir. En general, la probabilidad de hallar un partón con una fracción del momento $x P^{\mu}$ es una función de distribución $f\left(x, q^{2}\right)$. En el caso en que los partones son libres, esta distribución es independiente de $q^{2}$, es decir $f \equiv f(x)$. Este no es el caso de QCD, donde las funciones de distribución partónicas varían al aumentar $q^{2}$, ya que cada partón tiende a separarse en múltiples partones con menor $x$. En consecuencia, la estructura de un hadrón de QCD depende de $q^{2}$, con el número de partones aumentando y el valor promedio de $x$ disminuyendo al aumentar $q^{2}$.

\section{Dispersión de Compton}

Las amplitudes para la dispersión inelástica profunda pueden extraerse de la parte imaginaria de la dispersión de Compton. En particular, pueden extraerse del elemento de matriz de dos corrientes electromagnéticas dentro del hadrón de interés:

$$
\left(T_{\mu \nu}\right)_{h^{\prime} h}=i \int d^{4} x e^{i q . x}\left\langle P, h^{\prime}\left|T\left(j_{\mu}(x), j_{\nu}(0)\right)\right| P, h\right\rangle,
$$

donde $T\left(\hat{\mathcal{O}}_{1} \hat{\mathcal{O}}_{2}\right)$ indica un producto temporalmente ordenado entre $\hat{\mathcal{O}}_{1}$ y $\hat{\mathcal{O}}_{2}$. El tensor $T_{\mu \nu} \equiv$ $T_{\mu \nu}(P, q, s)$ tiene las mismas propiedades de simetría que $W_{\mu \nu}(P, q, s)$ y cumple con la condición derivada de la conservación de la corriente. Por lo tanto puede expandirse en términos de la misma estructura tensorial que $W_{\mu \nu}$.

El teorema óptico 49 implica que

$$
2 \operatorname{Im} \tilde{F}_{j}=F_{j},
$$

con $\tilde{F}_{j}$ la función de estructura $j$ de $T_{\mu \nu}$ y $F_{j}$ la de $W_{\mu \nu}$. 


\subsection{Funciones de estructura del hadrón}

Las funciones de estructura del hadrón se definen en términos del tensor hadrónico $W_{\mu \nu}$. Son funciones adimensionales que dependen de $P^{2}, P \cdot q$ y $q^{2}$. Suele escribírselas como funciones de las variables adimensionales $t=\frac{P^{2}}{q^{2}}$ y $x$. Estas funciones se obtienen escribiendo la descomposición más general posible para el tensor hadrónico en tensores invariantes de Lorentz $S O(1,3)$, satisfaciendo ciertas condiciones físicas. Las condiciones que debe satisfacer $W_{\mu \nu}$ para interacciones que conservan la simetría de paridad son

- La conservacion de la corriente, $\partial_{\mu} j^{\mu}(x)=0$.

- Las interacciones fuertes son invariantes bajo paridad.

- La invariancia ante inversión temporal.

- La invariancia ante traslaciones.

Con todo esto, saldrán las estructuras tensoriales específicas para hadrones de cada espín que detallaremos a continuación.

\section{Blancos hadrónicos de espín 0}

El tensor hadrónico más general para blancos hadrónicos de espín 0 e interacciones donde se conserva paridad puede escribirse en términos de $P$ y $q$ pidiendo que se cumplan las condiciones de simetría pertinentes. Suele escribirse $W_{\mu \nu}$ de la forma:

$$
W_{\mu \nu}=F_{1}\left(\eta_{\mu \nu}-\frac{q_{\mu} q_{\nu}}{q^{2}}\right)-\frac{F_{2}}{P \cdot q}\left(P_{\mu}-\frac{P \cdot q q_{\mu}}{q^{2}}\right)\left(P_{\nu}-\frac{P \cdot q q_{\nu}}{q^{2}}\right) .
$$

El coeficiente de cada uno de los términos tensoriales se llama función de estructura, es decir, $F_{1}$ y $F_{2}$ son las funciones de estructura en este caso. Como la corriente leptónica también se conserva, entonces se tiene $q_{\mu} l^{\mu \nu}=q_{\nu} l^{\mu \nu}=0$. Por ello, es conveniente simplificar la expresión para $W_{\mu \nu}$, omitiendo todos los términos que tengan $q_{\mu}$ y $q_{\nu}$, antes de ser contraido con $l^{\mu \nu}$ :

$$
W_{\mu \nu}=F_{1} \eta_{\mu \nu}-\frac{F_{2}}{P \cdot q} P_{\mu} P_{\nu}
$$

\section{Blancos hadrónicos de espín $\frac{1}{2}$}

El tensor hadrónico más general para blancos hadrónicos de espín $1 / 2$ e interacciones donde se conserva paridad puede escribirse en términos de $P, q, s$ y los tensores invariantes $\eta_{\alpha \beta}$ y $\epsilon_{\alpha \beta \lambda \sigma}$ pidiendo que se cumplan las condiciones de las condiciones de simetría pertinentes. Suele escribirse $W_{\mu \nu}$ de la forma:

$$
\begin{aligned}
W_{\mu \nu}= & F_{1}\left(\eta_{\mu \nu}-\frac{q_{\mu} q_{\nu}}{q^{2}}\right)-\frac{F_{2}}{P \cdot q}\left(P_{\mu}-\frac{P \cdot q q_{\mu}}{q^{2}}\right)\left(P_{\nu}-\frac{P \cdot q q_{\nu}}{q^{2}}\right) \\
& -\frac{i g_{1}}{P \cdot q} \epsilon_{\mu \nu \lambda \sigma} q^{\lambda} s^{\sigma}-\frac{i g_{2}}{(P \cdot q)^{2}} \epsilon_{\mu \nu \lambda \sigma} q^{\lambda}\left(P \cdot q s^{\sigma}-s \cdot q P^{\sigma}\right) .
\end{aligned}
$$

Las funciones de estructura son en este caso $F_{1}, F_{2}, g_{1}$ y $g_{2}$. Omitiendo todos los términos que tengan $q_{\mu}$ y $q_{\nu}$, resulta

$$
W_{\mu \nu}=F_{1} \eta_{\mu \nu}-\frac{F_{2}}{P \cdot q} P_{\mu} P_{\nu}-\frac{i g_{1}}{P \cdot q} \epsilon_{\mu \nu \lambda \sigma} q^{\lambda} s^{\sigma}-\frac{i g_{2}}{(P \cdot q)^{2}} \epsilon_{\mu \nu \lambda \sigma} q^{\lambda}\left(P \cdot q s^{\sigma}-s \cdot q P^{\sigma}\right) .
$$


Para un blanco de espín $\frac{1}{2}$, la parte simétrica de $W_{\mu \nu}$ es independiente del espín del hadrón, y la parte dependiente de dicho espín es antisimétrica en los índices $\mu$ y $\nu$. Como consecuencia, la combinación $l^{\mu \nu} W_{\mu \nu}$ no tiene términos que dependan sólo del espín hadrónico o del espín leptónico, todos los términos tienen ambos o ninguno. Por lo tanto, las funciones de estructura $F_{1}$ y $F_{2}$ pueden medirse usando un haz leptónico y un blanco no polarizados, pero para medir las funciones $g_{1}$ y $g_{2}$ se necesita que ambos estén polarizados. Esto no es cierto para blancos de espín mayor que $1 / 2$, como se verá para el caso de espín 1.

\section{Blancos hadrónicos de espín 1}

En [55], Hoodbhoy, Jaffe y Manohar calculan el tensor hadrónico para la dispersión inelástica profunda para blancos polarizados de espín 1 en interacciónes que conservan la simetría de paridad. Aparecen nuevas funciones de estructura adicionales al caso de espín 1/2. Dentro de este grupo pueden incluirse núcleos de espín 1, tales como el deuterio, el litio y el nitrógeno, así como también los mesones vectoriales, como es el caso del mesón $\rho$.

Las cuatro nuevas funciones de estructura, $b_{1}, b_{2}, b_{3}$ y $b_{4}$ aparecen en la parte simétrica de $W_{\mu \nu}$, de manera que contribuyen a la dispersión con un haz no polarizado. El tensor hadrónico más general puede escribirse en términos de ocho funciones de estructura independientes. Omitiendo todos los términos que contienen $q_{\mu}$ y $q_{\nu}$, se tiene la siguiente expresión para $W_{\mu \nu}$ :

$$
\begin{aligned}
W_{\mu \nu}= & F_{1} \eta_{\mu \nu}-\frac{F_{2}}{P \cdot q} P_{\mu} P_{\nu}+b_{1} r_{\mu \nu}-\frac{b_{2}}{6}\left(s_{\mu \nu}+t_{\mu \nu}+u_{\mu \nu}\right)-\frac{b_{3}}{2}\left(s_{\mu \nu}-u_{\mu \nu}\right) \\
& -\frac{b_{4}}{2}\left(s_{\mu \nu}-t_{\mu \nu}\right)-\frac{i g_{1}}{P \cdot q} \epsilon_{\mu \nu \lambda \sigma} q^{\lambda} s^{\sigma}-\frac{i g_{2}}{(P \cdot q)^{2}} \epsilon_{\mu \nu \lambda \sigma} q^{\lambda}\left(P \cdot q s^{\sigma}-s \cdot q P^{\sigma}\right),
\end{aligned}
$$

donde se usaron las siguientes definiciones:

$$
\begin{gathered}
r_{\mu \nu} \equiv \frac{1}{(P \cdot q)^{2}}\left(q \cdot \zeta^{*} q \cdot \zeta-\frac{1}{3}(P \cdot q)^{2} \kappa\right) \eta_{\mu \nu} \\
s_{\mu \nu} \equiv \frac{2}{(P \cdot q)^{3}}\left(q \cdot \zeta^{*} q \cdot \zeta-\frac{1}{3}(P \cdot q)^{2} \kappa\right) P_{\mu} P_{\nu} \\
t_{\mu \nu} \equiv \frac{1}{2(P \cdot q)^{2}}\left(q \cdot \zeta^{*} P_{\mu} \zeta_{\nu}+q \cdot \zeta^{*} P_{\nu} \zeta_{\mu}+q \cdot \zeta P_{\mu} \zeta_{\nu}^{*}+q . \zeta P_{\nu} \zeta_{\mu}^{*}-\frac{4}{3}(P \cdot q) P_{\mu} P_{\nu}\right) \\
u_{\mu \nu} \equiv \frac{1}{P \cdot q}\left(\zeta_{\mu}^{*} \zeta_{\nu}+\zeta_{\nu}^{*} \zeta_{\mu}-\frac{2}{3} M^{2} \eta_{\mu \nu}-\frac{2}{3} P_{\mu} P_{\nu}\right) \\
s^{\sigma} \equiv \frac{-i}{M^{2}} \epsilon^{\sigma \alpha \beta \rho} \zeta_{\alpha}^{*} \zeta_{\beta} P_{\rho},
\end{gathered}
$$

siendo $\kappa=1-4 x^{2} P^{2} / q^{2}$ y $s^{\sigma}$ un cuadrivector análogo al cuadrivector de espín para partículas de espín $\frac{1}{2}$ y $\zeta$ la polarización del blanco. Se cumple la condición $P \cdot \zeta=0$. La normalización está dada por:

$$
\zeta \cdot \zeta^{*}=M^{2}
$$




\section{Momentos de las funciones de estructura para espín 1}

El $n$-ésimo momento de la función $f$ se define como

$$
M_{n}(f) \equiv \int_{0}^{1} d x x^{n-1} f(x),
$$

Existen algunas desigualdades entre momentos que deben satisfacerse. Nos centraremos en el caso de espín $s=1$.

Del estudio de la sección eficaz $5^{5}$ puede verse que, para que esta sea positiva, debe cumplirse

$$
x y^{2}\left(F_{1}-\frac{b_{1}}{3}\right)+(1-y)\left(F_{2}-\frac{b_{2}}{3}\right) \geq x y(2-y)\left|g_{1}\right| \geq 0,
$$

para todo $x$ e $y$. En particular, para $y=1$ resulta que

$$
F_{1}-\frac{b_{1}}{3} \geq\left|g_{1}\right| \geq 0 \text {. }
$$

De aquí, y a partir de la definición de los momentos 4.3.12, resulta

$$
\begin{aligned}
\left|M_{n}\left(g_{1}\right)\right| & =\left|\int_{0}^{1} d x x^{n-1} g_{1}(x)\right| \leq \int_{0}^{1} d x x^{n-1}\left|g_{1}(x)\right| \\
& \leq \int_{0}^{1} d x x^{n-1}\left(F_{1}(x)-\frac{b_{1}(x)}{3}\right)=M_{n}\left(F_{1}\right)-\frac{1}{3} M_{n}\left(b_{1}\right),
\end{aligned}
$$

Por otro lado, al ser $F_{1} \geq 0$ y $0 \leq x \leq 1$,

$$
M_{n}\left(F_{1}\right)=\int_{0}^{1} d x x^{n-1} F_{1}(x) \geq \int_{0}^{1} d x x^{n-1} x F_{1}(x)=M_{n+1}\left(F_{1}\right),
$$

Con esto hemos demostrado la cadena de desigualdades

$$
M_{1}\left(F_{1}\right) \geq M_{2}\left(F_{1}\right) \geq M_{3}\left(F_{1}\right) \geq M_{4}\left(F_{1}\right) \ldots
$$

así como

$$
M_{1}\left(F_{1}\right) \geq \frac{1}{3} M_{1}\left(b_{1}\right)+\left|M_{1}\left(g_{1}\right)\right|, \quad M_{2}\left(F_{1}\right) \geq \frac{1}{3} M_{2}\left(b_{1}\right)+\left|M_{2}\left(g_{1}\right)\right|, \quad \ldots
$$

\subsection{Expansión en producto de operadores (OPE)}

La expansión en producto de operadores (OPE, del inglés Operator Product Expansion) es una forma de descomponer las funciones de estructura para estudiar su comportamiento directamente desde $Q C D$, es decir sin considerar un modelo en particular. Comenzamos por el producto temporalmente ordenado de dos corrientes

$$
\hat{T}_{\mu \nu} \equiv i \int d^{4} x e^{i q \cdot x} T\left(j_{\mu}(x) j_{\nu}(0)\right) .
$$

Este elemento de matriz $\hat{T}_{\mu \nu}$ da la amplitud Compton

$$
\left(T_{\mu \nu}\right)_{\lambda^{\prime} \lambda}=\left\langle p, \lambda^{\prime}\left|\hat{T}_{\mu \nu}\right| p, \lambda\right\rangle .
$$

\footnotetext{
${ }^{5}$ Este estudio se realizará en detalle en la Sección 8.2 .
} 
que se relaciona con el tensor hadrónico como hemos visto en la Sección anterior. Nos interesa calcular $T_{\mu \nu}$. Consideremos el producto de dos operadores locales

$$
\mathcal{O}_{a}(z) \mathcal{O}_{b}(0)
$$

En el límite $z \rightarrow 0$, estos operadores están prácticamente en el mismo punto. En este límite el producto de operadores se puede escribir como una expansión en operadores locales,

$$
\lim _{z \rightarrow 0} \mathcal{O}_{a}(z) \mathcal{O}_{b}(0)=\sum_{k} c_{a b k}(z) \mathcal{O}_{k}(0)
$$

Los coeficientes $c_{a b k}$ dependen de la separación $z$ entre los operadores. Esta igualdad vale siempre y cuando el producto de operadores se pruebe a distancias mayores que $z$. Podemos reemplazar entonces el producto $\mathcal{O}_{a}(x) \mathcal{O}_{b}(0)$ en el cálculo de elementos de matriz a través de la expansión 4.4.4 donde los coeficientes $c_{a b k}(z)$ son independientes de los elementos de matriz, siempre que los estados externos tengan componentes de momento pequeñas comparadas con $z$. La constante de acoplamiento en QCD es pequeña para distancias cortas debido a la libertad asintótica. Es por esto que las funciones coeficientes pueden calcularse en teoría de perturbaciones, pues los efectos no perturbativos ocurrirán a escalas mucho mayores a $z$, no afectando entonces el cálculo de estas funciones.

La versión del OPE en el espacio de momentos es para el producto

$$
\int d^{4} z e^{i q \cdot z} \mathcal{O}_{a}(z) \mathcal{O}_{b}(0)
$$

En el límite $q \rightarrow \infty$, la transformada de Fourier en 4.4.1 fuerza a que $z \rightarrow 0$, y nuevamente el producto de operadores se puede expandir en términos de operadores locales con funciones coeficientes dependiendo de $q$,

$$
\lim _{q \rightarrow \infty} \int d^{4} z e^{i q \cdot z} \mathcal{O}_{a}(z) \mathcal{O}_{b}(0)=\sum_{k} c_{a b k}(q) \mathcal{O}_{k}(0) .
$$

Esta expresión vale para todos los elementos de matriz siempre que $q \gg p_{i}$ donde $p_{i}$ son los momentos de los estados externos.

Usaremos la versión (4.4.6) del OPE. El producto de dos corrientes electromagnéticas en (4.4.1) puede expandirse en términos de una suma de operadores locales multiplicados por coeficientes que son funciones de $q$. Esta expansión es válida para elementos de matriz en el blanco, 4.4.2), siempre que $q \gg \Lambda_{Q C D}$. Los operadores locales en este OPE para QCD son los de quarks y gluones con dimensión arbitraria $d$ y espín $n$. Un operador tal se escribe como

$$
\mathcal{O}_{d, n}^{\mu_{1} \ldots \mu_{n}},
$$

donde $\mathcal{O}_{d, n}$ es simétrico y de traza nula en $\mu_{1} \ldots \mu_{n}$. El elemento de matriz de $\mathcal{O}_{d, n}$ en el blanco hadrónico es proporcional a

$$
M^{d-n-2} \mathcal{S}\left[p^{\mu_{1}} \ldots p^{\mu_{n}}\right]
$$

para un operador vectorial y a

$$
M^{d-n-2} \mathcal{S}\left[s^{\mu_{1}} p^{\mu_{2}} \ldots p^{\mu_{n}}\right]
$$

para uno axial. $\mathcal{S}$ actúa sobre un tensor para proyectar la componente totalmente simétrica y de traza nula. La potencia de $M$ proviene del análisis dimensional, pues un estado hadrónico con la normalización relativista usual tiene dimensión $d=-1$. Las funciones coeficiente en el OPE 
son funciones solo de $q$. Por esto, los índices libres en el operador $\mathcal{O}$ deben ser bien $\mu, \nu$ o bien estar contraídas con $q^{\alpha}$. Cada índice de $\mathcal{O}$ contraído con $q^{\alpha}$ produce un factor $p \cdot q$ o $s \cdot q$, que es de orden $\frac{Q^{2}}{M}$ en el límite de DIS. Un índice $\mu$ o $\nu$ se contrae con el tensor leptónico y produce un factor $p \cdot k, p \cdot k^{\prime}, s \cdot k$ o $s \cdot k^{\prime}$, todos estos también de orden $\frac{Q^{2}}{M}$ en el límite de DIS. Por otro lado, dado que $\hat{T}_{\mu \nu}$ tiene dimensión dos, el coeficiente de $\mathcal{O}$ debe tener dimensión $Q^{2-d}$ en el OPE. Entonces la contribución de cualquier operador $\mathcal{O}$ a la sección eficaz $W_{\mu \nu} \ell^{\mu \nu}$ es de orden

$$
\begin{aligned}
c_{\mu_{1} \ldots \mu_{n}} \mathcal{O}_{d, n}^{\mu_{1} \ldots \mu_{n}} & \rightarrow \frac{q_{\mu_{1}}}{Q} \ldots \frac{q_{\mu_{n}}}{Q} Q^{2-d}\left\langle\mathcal{O}_{d}^{\mu_{1} \ldots \mu_{n}}\right\rangle \\
& \rightarrow \frac{q_{\mu_{1}}}{Q} \ldots \frac{q_{\mu_{n}}}{Q} Q^{2-d} M^{d-n-2} p^{\mu_{1}} \ldots p^{\mu_{n}} \\
& \rightarrow \frac{(p \cdot q)^{n}}{Q^{n}} Q^{2-d} M^{d-n-2} \\
& \rightarrow \omega^{n}\left(\frac{Q}{M}\right)^{2+n-d}=\omega^{n}\left(\frac{Q}{M}\right)^{2-t}
\end{aligned}
$$

donde el twist $t$ se define como

$$
\text { twist }=t=d-n=\text { dimensión }- \text { espín. }
$$

Los operadores más importantes en el OPE son los de menor twist. Los operadores de $t=2$ aportan una contribución finita a las funciones de estructura en el límite de DIS, los de $t=3$ son suprimidos como $\frac{Q}{M}$, etc. Los campos fundamentales en QCD son quarks y gluones, por lo que los operadores en el OPE pueden escribirse en términos de campos de quarks $\psi$, tensores de campo de gluones $G_{\mu \nu}$ y la derivada covariante $D^{\mu}$.

Cualquier operador invariante de gauge debe contener al menos dos campos de quarks, o bien dos tensores de campo de gluones. Con esto, el menor twist posible es $t=2$. Un operador con este twist tiene bien dos $\psi$ 's o bien dos $G_{\mu \nu}$ 's y un número arbitrario de derivadas covariantes. Los índices de las derivadas covariantes no están contraídos, pues un operador como $D^{2}$ tendría $t=2$, mientras que $D^{\alpha} D^{\beta}$ tiene $t=0$.

En [5] se muestra que para un proceso de DIS en el acoplamiento fuerte los momentos vienen dados por

$$
\begin{gathered}
M_{n}^{(s)}\left(q^{2}\right) \approx \frac{1}{4} \sum_{j}^{\prime} C_{n, j}^{(s)} A_{n, j}\left(\frac{\Lambda^{2}}{q^{2}}\right)^{\frac{1}{2} \tau_{n, j}-1}+\frac{1}{4} \sum_{\mathcal{Q}_{p}=\mathcal{Q}}{ }^{\prime \prime} C_{n, p}^{(s)} A_{n, p}\left(\frac{\Lambda^{2}}{q^{2}}\right)^{\tau_{p}-1} \\
+\frac{1}{4 N^{2}} \sum_{\mathcal{Q}_{p} \neq \mathcal{Q}}{ }^{\prime \prime} C_{n, p}^{(s)} a_{n, p}\left(\frac{\Lambda^{2}}{q^{2}}\right)^{\tau_{p}-1} .
\end{gathered}
$$

De aquí se ve que, mientras el primer término domina para $\lambda$ pequeño, para valores grandes de $\lambda$ esto se vuelve más complejo. Para $\Lambda^{2} \lesssim q^{2}$ el segundo término domina siempre, pero decae como $\left(q^{2}\right)^{-\tau_{\mathcal{Q}}+1}$, donde $\tau_{\mathcal{Q}}$ es el mínimo twist de los operadores $\mathcal{P}_{p}$ de carga $\mathcal{Q}$. Típicamente $\tau_{\mathcal{Q}} \sim \mathcal{Q}$; como por ejemplo si todos los campos involucrados tienen carga 0 o 1 . En cambio, si $\tau_{\mathcal{Q}} \neq \tau_{\mathrm{c}}$, donde $\tau_{\mathrm{c}}$ es el twist mínimo de los operadores cargados $\mathcal{P}_{p}$, habrá otra transición. Para $\Lambda^{2} N^{2 /\left(\tau_{\mathcal{Q}}-\tau_{\mathrm{c}}\right)} \lesssim q^{2}$ el tercer término se vuelve el dominante: la contribución del operador $\mathcal{P}_{p}$ con twist más bajo $\left(\tau_{p}=\tau_{\mathrm{c}}\right)$ cae como $\left(q^{2}\right)^{-\tau_{\mathrm{c}}+1}$, con lo que su supresión global de orden $\frac{1}{N^{2}}$ domina la amplitud para $q^{2} \rightarrow-\infty$. 


\section{Capítulo 5}

\section{Modelos holográficos duales de teorías de YM confinantes}

En este Capítulo describiremos los modelos holográficos duales de teorías de gauge confinantes en el límite planar y en $3+1$ dimensiones dentro de los cuales estudiamos el proceso de DIS. Como se verá todos estos modelos se derivan de las teorías de supercuerdas del tipo IIA y IIB, es decir que son modelos del tipo top-down, por lo que contienen grados de libertad fundamentales de la teoría de cuerdas. En la Sección 5.1 comenzamos con el modelo dual a la teoría $\mathcal{N}=1^{*}$ SYM en el límite planar, desarrollado por Joseph Polchinski y Matthew J. Strassler en la referencia [11]. A partir de este modelo, los mismos autores desarrollaron el método descripto en [5] para estudiar el DIS de glueballs holográficos, que son duales a dilatones en el interior del modelo de supergravedad. El cálculo explícito de DIS en este modelo se explica en detalle en las Secciones 6.1 y 7.1. Este modelo no posee D $p$-branas de sabor, por lo que las partículas blanco del DIS (los glueballs) están en la representación adjunta del grupo de gauge.

En la Sección 5.2 estudiaremos modelos que incluyen $\mathrm{D} p$-branas de sabor. Este estudio fue iniciado por Karch y Katz [56], quienes demostraron que introduciendo $N_{f} \mathrm{D} p$-branas, en la aproximación de prueba en la que $N_{f} \ll N$ pueden definirse mesones holográficos en la teoría holográfica dual, que corresponden a excitaciones sobre las $\mathrm{D} p$-branas. En particular, estudiaremos tres modelos con estas características. En la Subsección 5.2.1 introducimos el modelo de Kruczenski, Mateos, Myers y Winters [12 consistente en un fondo de $N$ D3 branas y $N_{f}$ D7-branas de prueba, en el contexto de supergravedad tipo IIB. En la Subsección 5.2.2 estudiamos un modelo de los mismos autores [13] en el que estudian un fondo con $N$ D4 branas y $N_{f}$ pares de D6 $\overline{\mathrm{D} 6}$ branas de prueba en supergravedad tipo IIA. Finalmente, en la Sección 5.2.3 vemos una importante evolución de este último modelo, que lo acerca más a una descripción holográfica dual del límite planar de QCD, correspondiente al modelo de Sakai y Sugimoto [14, consistente en un fondo con $N$ D4 branas y $N_{f}$ pares de D8 $\overline{\mathrm{D} 8}$ branas de prueba, también en supergravedad tipo IIA.

\subsection{Modelo holográfico de Polchinski y Strassler}

\section{Características del modelo}

Estamos interesados en el proceso de DIS porque permite estudiar la estructura interna de los hadrones. En el trabajo [5] se estudia el régimen de teoría de cuerdas débilmente acoplada y se lo trata de interpolar hasta el régimen de teoría de gauge débilmente acoplada, que corresponde al extremo opuesto del valor del parámetro de 't Hooft $\lambda=g_{s} N$. Pondremos el foco en teorías 
de gauge confinantes que sean invariantes de escala (al menos aproximadamente, para $q \gg \Lambda$, donde $q$ es el momento de la partícula intercambiada y $\Lambda$ es alguna escala característica de la teoría). Estas teorías se dividen entre las que son débilmente y las fuertemente acopladas a altas energías. El primer caso incluye las teorías asintóticamente libres como $S U(N), Q C D$ y $\mathcal{N}=4 \mathrm{SYM}]^{1} \mathrm{La}$ invariancia de escala de QCD suele violarse por términos logarítmicos, que no son importantes para $P \gg \Lambda$. Las teorías invariantes de escala fuertemente acopladas a altas energías, en cambio, no pueden describirse con la teoría de perturbaciones en ninguna escala y, en principio, pueden describirse mediante un modelo dual basado en la teoría de cuerdas.

Describiremos en esta Sección la llamada teoría $\mathcal{N}=1^{*} \mathrm{SYM}$. Esta es la teoría resultante de partir de $\mathcal{N}=4 \mathrm{SYM}$ y romper explícitamente la supersimetría hasta $\mathcal{N}=1$ a una escala de masa arbitraria $m$, que se impone para los escalares del multiplete de gauge. Esta teoría contiene los mismos campos no masivos que una teoría de gauge confinante asintóticamente libre, mientras que los escalares y fermiones masivos regulan la teoría en el UV.

La dualidad original de Maldacena se aplica para la teoría de gauge conforme $\mathcal{N}=4 \mathrm{SYM}$ -dual a $A d S_{5} \times S^{5}$ - que no es confinante. Para conseguir una teoría confinante a partir de esta debemos deformarla agregando términos de masa, que pueden preservar o no algunas de las supersimetrías. La teoría $\mathcal{N}=1^{*}$ descripta en [11 constituye el primer ejemplo de una descripción dual de supergravedad para una teoría de gauge confinante en cuatro dimensiones. Posteriormente se han desarrollado otros ejemplos como los modelos de Klebanov-Strassler [57] y Maldacena-Núñez [58].

La idea es concentrar la atención en el caso de teorías que preserven una supersimetría $\mathcal{N}=1$, aunque las soluciones encontradas son estables aún rompiendo la supersimetría completamente. El multiplete vectorial $\mathcal{N}=4$ está compuesto por un multiplete vectorial $\mathcal{N}=1 \mathrm{y}$ tres multipletes quirales $\mathcal{N}=1$. La propuesta en [11] es agregar masas a estos tres últimos, de manera que preserven la supersimetría [11]. Si bien esta teoría ya había sido estudiada previamente, es en la referencia citada donde se demuestra que la perturbación así introducida no hace aparecer una singularidad desnuda, sino una fuente expandida de branas, lo que hace calculables las cantidades físicas. Esta teoría contiene fases conformes en la misma clase de universalidad que $\mathcal{N}=1$ SYM.

Si quisiéramos estudiar la teoría pura $\mathcal{N}=1 \mathrm{SYM}$ o $\mathcal{N}=0$ deberíamos trabajar con valores pequeños del acoplamiento de 't Hooft, y por ende llevando las masas de los nuevos multipletes a infinito. Como queremos trabajar en el régimen donde la teoría de supergravedad es válida, es decir a valores pequeños del parámetro de 't Hooft, estas masas deben mantenerse finitos. Estos multipletes son en cuatro dimensiones, y la teoría en el UV resultará conforme. Los dos regímenes extremos de esta teoría son: i) $m \sim \Lambda$ : En este régimen tenemos una teoría con $\lambda$ grande en el UV, que es débilmente acoplada en teoría de cuerdas y ii) $m \gg \Lambda$ : Una teoría con $\lambda$ pequeño en el UV, que resulta en una teoría cuántica de campos perturbativa para $\mu>\Lambda$. En la Figura 5.1 puede verse el corrimiento (running) de la constante de acoplamiento con la energía para los dos casos mencionados, así como una comparación con la teoría $\mathcal{N}=1 \mathrm{SYM}$ pura.

En la referencia citada se concluye que para un acoplamiento de 't Hooft $\lambda$ pequeño, donde puede aplicarse la teoría de campos perturbativa, la estructura hadrónica es similar a la de QCD. Aquí, cada hadrón está compuesto de un pequeño número de partones que contienen la mayor parte de la energía, rodeados por una nube de partones más pequeños, con una fracción de momento $x$ más pequeña. En el régimen de supergravedad, donde $\lambda$ es grande, el hadrón está compuesto de muchos partones pequeños, y por ende la probabilidad de encontrar un partón con una fracción considerable de la energía total es despreciable. Para el régimen $q^{2} \rightarrow \infty$ con $N$ y $x$ fijos el leptón es dispersado por uno de los pequeños hadrones de la nube, pues los partones

\footnotetext{
${ }^{1}$ Notar que $\mathcal{N}=4$ SYM siempre puede estudiarse en su límite de acoplamiento débil mediante pQFT.
} 


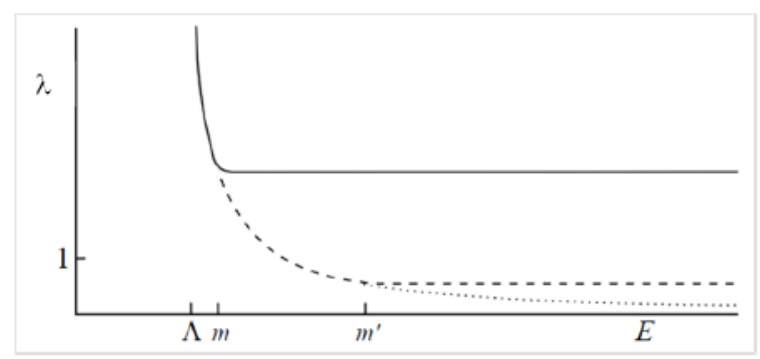

Figura 5.1: Corrimiento de la constante de acoplamiento $\lambda=g_{Y M}^{2} N$ con la energía. Se grafican los casos $m \sim \Lambda$ (línea continua), $m^{\prime} \gg \Lambda$ (línea de trazos), ambos de la teoría $\mathcal{N}=1^{*}$, así como la teoría $\mathcal{N}=1$, asintóticamente libre (línea punteada). Figura tomada de [5] .

que conforman el hadrón están demasiado fragmentados.

\section{Fondo de supergravedad}

En esta Sección describiremos el fondo de supergravedad dual a la teoría $\mathcal{N}=1^{*} \mathrm{SYM}$, siguiendo las convenciones de Polchinski y Strassler [5]. En el Capítulo 6 utilizaremos esta descripción para calcular las funciones de estructura.

Dada una teoría de gauge conforme, el modelo dual de teoría de cuerdas está en un espacio de la forma $A d S_{5} \times W$, donde $W$ puede ser $S^{5}$ u otro espacio de Einstein compacto de cinco dimensiones. La métrica más general es

$$
d s^{2}=\frac{r^{2}}{R^{2}} \eta_{\mu \nu} d y^{\mu} d y^{\nu}+\frac{R^{2}}{r^{2}} d r^{2}+R^{2} \widehat{d s}_{W}^{2}
$$

donde $R=\left(4 \pi g_{s} N \alpha^{2}\right)^{1 / 4}=\left(4 \pi \lambda \alpha^{2}\right)^{1 / 4}$ es el radio del $A d S_{5}$, así como de la esfera $S^{5}$ en el caso de que sea $W=S^{5}$. Las coordenadas $y^{\mu}$ serán las que identificaremos con la teoría de gauge, mientras que $r$ será la coordenada radial holográfica, y las coordenadas dentro del espacio $W$ serán denotadas genéricamente con $\Omega$.

Es importante notar aquí el papel del corrimiento al rojo gravitatorio (gravitational redshift) debido al factor de deformación (warp factor) $g_{t t}=\frac{r^{2}}{R^{2}}$ frente a la parte tetradimensional de la métrica. El momento de esta parte de la métrica, $p_{\mu}=-i \partial_{\mu}$ con $\mu=0, \ldots, 3$, vistos por un observador inercial en diez dimensiones es

$$
\tilde{p}_{\mu}=\frac{R}{r} p_{\mu} .
$$

La escala de energía característica en diez dimensiones es, por análisis dimensional, $\tilde{E}=R^{-1}$. Por ende, la energía en cuatro dimensiones es $E \sim \frac{r}{R^{2}}$, de lo que resulta $E(r=0) \rightarrow 0$ en el horizonte y $E(r=\infty) \rightarrow \infty$ en el borde del $A d S_{5}$.

Si la teoría es confinante la métrica será (5.1.1) solamente de manera asintótica para grandes valores de $r$, mientras que será diferente para radios del orden de

$$
r_{0} \equiv \Lambda R^{2},
$$

donde $\Lambda$ es la masa del estado de glueball más liviano y nos definirá una escala característica de energía (mass gap). Esto protege al warp factor de anularse. 
Trabajaremos siempre con interacciones que sucedan para valores de $q$ suficientemente grandes, tal que ${ }^{2}$

$$
q_{\text {int }} \gg \Lambda \Rightarrow r_{\text {int }}=q_{\text {int }} R^{2} \gg \Lambda R^{2}=r_{0},
$$

y por lo tanto no nos importará la física en regiones cercanas a este cutoff $r_{0}$. Esta teoría resultante será similar a QCD, asintóticamente conforme en el UV, con confinamiento de color y mass gap en el IR, pero fuertemente acoplada, lo que en definitiva diferenciará a esta descripción de QCD planar.

Todo lo dicho hasta aquí vale para un gran número de modelos, desde $\mathcal{N}=1^{*} \mathrm{SYM}$ [11], el modelo D3D7 [12] y las teorías que incluyen una cascada de dualidades de Seiberg como [57]. Un punto fundamental es que el modelo en cuestión posea una simetría global que contenga a $U(1)$ como un subgrupo, lo que permitirá realizar la interacción electromagnética en el modelo dual $\sqrt[3]{3}$

\section{Limitaciones del modelo}

En el trabajo de Polchinski y Strassler aquí descripto [5], si bien se desarrolla un método para tratar el problema del DIS desde una perspectiva holográfica - tanto en el rango $x \lesssim 1$ como $x \ll 1$, como estudiaremos en las Secciones 6.1 y 7.1 no se incluyen campos de materia en la representación fundamental del grupo de gauge. Esto se logrará agregando D $p$-branas de sabor (flavor) al modelo.

En la siguiente Sección describiremos los modelos más relevantes al presente caso que incluyen estos ingredientes y, en la Segunda Parte de la Tesis extenderemos en forma no trivial, desde primeros principios, el método desarrollado por Polchinski y Strassler a esta clase de modelos, ampliando considerablemente su rango de acción.

\subsection{Modelos con branas de sabor}

Los modelos de gravedad duales a una teoría de gauge SYM pura, como el caso de la conjetura original de Maldacena [23. o la teoría $\mathcal{N}=1^{*}$ descripta en la última Sección, dan lugar solamente a cuerdas cerradas, duales a campos que transforman en la representación adjunta del grupo de gauge. Al agregar $N_{f} \mathrm{D} p$-branas de prueba en el modelo de cuerdas que se extiendan al menos en las cinco dimensiones correspondientes al $A d S_{5}$, esto da lugar a un sector de cuerdas abiertas, correspondiente a campos que transforman en la representación fundamental del grupo de gauge [56]. En el límite en que el número de $\mathrm{D} p$-branas de prueba agregadas es mucho menor que el número de D3-branas del fondo, $N_{f} \ll N$, la reacción de aquellas sobre el fondo de gravedad (backreaction) puede despreciarse. Esto se debe a que la intensidad con la que las D-branas hacen de fuente para la métrica es $g_{s} N_{f}$, y estamos en el límite en que $g_{s} \rightarrow 0$ mientras $g_{s} N_{f} \rightarrow 0$ y $\lambda=g_{s} N \rightarrow$ cte. Esta última condición nos dice que la reacción de las D3-branas sí será importante en la geometría, y por ende las reeemplazamos por su geometría de horizonte cercano. Las condiciones para que estos modelos sean viables son que las D $p$-branas sean estables y que estén libres de tadpoles. Esto se logra en [56] enrollando estas D $p$-branas en ciclos topológicamente triviales con flujo cero, de manera que no posean carga en el espacio

\footnotetext{
${ }^{2}$ Justificaremos esta implicación luego de la ecuación 6.1.18.

${ }^{3}$ Los modelos $D 4 D 8 \overline{D 8}[14$ y $D 4 D 6 \overline{D 6}$ [13] no cumplen estrictamente con lo que describimos anteriormente por no ser conformes en el límite UV. Igualmente serán analizados con un formalismo muy similar al presente en los Capítulos 6 y 7 pues cumplen con la hipótesis de poseer el subgrupo de simetría global $U(1)$, la cual es instrumental para la aplicación del razonamiento que utilizamos para obtener la versión holográfica dual del DIS.
} 
de cinco dimensiones. Más allá de los modelos estudiados en esta Tesis han habido adiciones de branas de sabor al modelo de Klebanov-Strassler [57] como [59, 60, 61], y al modelo de Maldacena-Núñez [58] como [62]. Las técnicas que describiremos para la segunda parte de la Tesis son aptas para ser usadas en estos modelos también y es esperable la obtención de resultados similares a los contenidos en esta Tesis más allá de los parámetros propios de cada geometría o modelo holográfico dual considerado.

\subsubsection{Modelo D3D7}

En esta Sección comenzaremos dando una breve introducción al modelo de D3D7-branas desarrollado en [12]. En [56] se demostró que introduciendo $N_{f}$ D7-branas en el fondo $A d S_{5} \times S^{5}$ de la teoría de cuerdas tipo IIB pueden describirse $N_{f}$ hipermultipletes en la teoría de gauge que preserven $\mathcal{N}=2$ supersimetrías en cuatro dimensiones. Partimos de un conjunto de $N$ D3branas paralelas coincidentes y de $N_{f}$ D7-branas que comparten con las primeras las direcciones 0-3. Los hipermultipletes de la teoría de gauge aparecen de los modos más livianos de cuerdas fundamentales extendidas entre las D3 y las D7-branas: los modos del tipo 3-7 y 7-3, con masa $m_{q}=L / 2 \pi \alpha^{\prime}$, donde $L$ es la distancia entre las D3 y las D7-branas en el plano $(8,9)$. En el límite de desacoplamiento de las D3-branas, $\lambda=g_{s} N \gg 1$ donde $g_{s}$ es el acoplamiento de la teoría de cuerdas, el fondo se convierte en $A d S_{5} \times S^{5}$. Por otro lado, si $N \gg N_{f}$ la reacción (backreaction) de las D7-branas puede despreciarse. Tomando estos límites, la descripción corresponde a $N_{f}$ D7-branas de prueba en una geometría $A d S_{5} \times S^{5}$.

Esta teoría tiene quarks dinámicos, por lo que es muy interesante investigar la estructura de DIS para mesones escalares y vectoriales que surge de este modelo. Resaltemos que este modelo no permite describir la ruptura espontánea de simetría quiral, lo que hace una importante diferencia con QCD. En la Sección 5.2.3 describiremos el modelo de Sakai y Sugimoto [14, que sí presenta esta ruptura. En la Segunda Parte de esta Tesis utilizaremos este modelo para calcular DIS a partir de los mesones escalares (Sección 6.2) y vectoriales (Sección 6.3) en el caso abeliano, correspondiente a $N_{f}=1$ ), lo que implica la existencia de un grupo de simetría global $U(1)$, asociado con la simetría de sabor de la teoría $\mathcal{N}=2 S U(N)$ SYM. En la Sección 6.4 extenderemos este cálculo a otros modelos, como por ejemplo el de Sakai y Sugimoto [14, mientras que en la Sección 6.5. finalmente, estudiaremos el caso de mesones vectoriales no abelianos, es decir con $N_{f}>1$, para todos los modelos estudiados. Además, obtenemos lagrangianos efectivos al orden subdominante en la expansión en $\frac{1}{N}$ y $\frac{N_{f}}{N}$.

\section{Características del modelo}

Haremos una breve introducción a las características que nos serán de utilidad del modelo D3D7. Seguiremos aquí la notación de la referencia [12]. Consideremos inicialmente una solución de teoría de supercuerdas tipo IIB con $N$ D3-branas coincidentes. Este fondo (background) corresponde a una geometría $A d S_{5} \times S^{5}$, cuya métrica es

$$
d s^{2}=\frac{r^{2}}{R^{2}} \eta^{\mu \nu} d y^{\mu} d y^{\nu}+\frac{R^{2}}{r^{2}} d \vec{Z} \cdot d \vec{Z}
$$

donde las coordenadas $Z^{i}, i=1, \ldots, 6$ representan las direcciones transversales a las D3-branas y $r=|\vec{Z}|$ es la coordenada radial. El radio del espacio $A d S_{5}$ (y también de la esfera $S^{5}$ ) será $R=\left(4 \pi g_{s} N \alpha^{2}\right)^{1 / 4}$.

Agreguemos ahora una D7-brana de prueba $4^{4}$ a una distancia $|\vec{Z}|=r_{D 7}=L$ de las D3-branas en la dirección del plano $(8,9)$. Para $L>0$ el hipermultiplete se vuelve masivo y por ende la

\footnotetext{
${ }^{4}$ La métrica será similar para $N_{f}$ D7-branas, siempre que sigamos en la aproximación de prueba $N_{f} \ll N$.
} 
simetría $R$ se reduce ahora a $S U(2)_{R}$. La métrica inducida en la D7-brana es

$$
d s^{2}=\frac{\rho^{2}+L^{2}}{R^{2}} \eta^{\mu \nu} d y^{\mu} d y^{\nu}+\frac{R^{2}}{\rho^{2}+L^{2}} d \rho^{2}+\frac{R^{2} \rho^{2}}{\rho^{2}+L^{2}} d \Omega_{3}^{2},
$$

donde definimos $r^{2}=L^{2}+\rho^{2}$ y $\Omega_{3}$ son las coordenadas angulares de la variedad generada por las coordenadas $4, \ldots, 7$. Para $L=0$ esta métrica se reduce a $A d S_{5} \times S^{3}$, dando lugar a una teoría dual de gauge conforme. Si $L \neq 0$, en cambio, esta métrica es solo asintóticamente $A d S_{5} \times S^{3}$ para $\rho \gg L$. Vemos aquí la ruptura explícita de la simetría conforme inducida por la masa del hipermultiplete $m_{q}=\frac{L}{2 \pi \alpha^{\prime}}$, que se recupera en el límite de altas energías $E \gg m_{q}$. En este límite la métrica asintótica

$$
d s^{2}=\frac{\rho^{2}}{R^{2}} \eta^{\mu \nu} d y^{\mu} d y^{\nu}+\frac{R^{2}}{\rho^{2}} d \rho^{2}+R^{2} d \Omega_{3}^{2} .
$$

Con esto, la distribución de branas resultantes es

$$
\begin{aligned}
& N \quad D 3: 00123------ \\
& N_{f} \quad D 7: 012345567-- \text {. }
\end{aligned}
$$

El espectro de mesones escalares y vectoriales fue calculado en [12]. Puede verse cómo estos se acomodan en supermultipletes que transforman respecto de las representaciones del grupo de simetría local $S U(2)_{R} \times S U(2)_{L}$. Estos mesones corresponden a excitaciones de cuerdas abiertas de la D7-brana. La dinámica de las fluctuaciones de la D7-brana de prueba está descripta a partir de la acción que es suma de la acción de Dirac-Born-Infeld y la de Wess-Zumino

$$
\begin{aligned}
S_{D 7} & =-\mu_{7} \int d^{8} \xi \sqrt{-\operatorname{det}\left(P[g]_{a b}+2 \pi \alpha^{\prime} F_{a b}\right)}+\frac{\left(2 \pi \alpha^{\prime}\right)^{2}}{2} \mu_{7} \int P\left[C^{(4)}\right] \wedge F \wedge F \\
& =S_{D B I}+S_{W Z} .
\end{aligned}
$$

Aquí $g_{a b}$ es la métrica $(5.2 .2), \mu_{7}=\left[(2 \pi)^{7} g_{s} \alpha^{4}\right]^{-1}$ es la tensión de la D7-brana y $P$ denota el pullback de los campos de fondo sobre el volumen de mundo de la D7-brana. La parte relevante del potencial de Ramond-Ramond en el término de Wess-Zumino es

$$
C^{(4)}=\frac{r^{4}}{R^{4}} d y^{0} \wedge d y^{1} \wedge d y^{2} \wedge d y^{3} .
$$

\subsubsection{Modelo D4D6 $\overline{\mathrm{D} 6}$}

En esta Sección describiremos el modelo consistente en $N$ D4 y $N_{f}$ D6-branas definido en [13. En este modelo, las D-branas vienen extendidas en la siguiente configuración:

$$
\begin{array}{cccccc}
N & D 4: & 0 & 1234 & - & ---- \\
N_{f} & D 6: & 0 & 123-56 & 7--
\end{array}
$$

Notemos que los dos conjuntos de D-branas pueden estar separadas entre sí en las direcciones $x_{8}$ y $x_{9}$. En el límite de desacoplamiento para las D4-branas este sistema da una versión no conforme de la correspondencia $A d S / d C F T$. Esto significa que del lado de la teoría de campos hay una teoría de gauge $S U(N)$ supersimétrica en cinco dimensiones acoplada con un defecto de cuatro dimensiones. El sistema es supersimétrico con $\mathcal{N}=2$ en cuatro dimensiones. Los grados de libertad localizados en el defecto son $N_{f}$ hipermultipletes en la representación fundamental de $S U(N)$, que proviene de las cuerdas abiertas que conectan las D4 con las D6-branas. Cada 
hipermultiplete consiste de dos fermiones de Weyl de quiralidades opuestas $\psi_{L}$ y $\psi_{R}$, y de dos escalares complejos.

Identificando la dirección 4 como $x_{4} \sim x_{4}+\frac{2 \pi}{M_{K K}}$ - donde $M_{K K}$ es la escala de masa para los modos de Kaluza-Klein - e imponiendo condiciones antiperiódicas para los fermiones en la D4-brana, todas las supersimetrías se rompen $\left(\mathcal{N}=1^{*}\right)$ y la teoría se vuelve 4-dimensional para energías $E \ll M_{K K}$. En el límite $N_{f} \ll N$ la backreaction de las D6-branas en el fondo de supergravedad es despreciable y por ende pueden tratarse estas como D-branas de prueba. En la descripción de cuerdas, la simetría $U(1)_{A}$ corresponde a la simetría de rotación en el plano 89.

\section{El fondo de D4D6-branas}

El fondo de supergravedad IIA dual a $N$ D4-branas compactificadas en una circunferencia con condiciones de contorno antiperiódicas para los fermiones toma la forma [13]

$$
\begin{aligned}
& d s^{2}=\left(\frac{U}{R}\right)^{3 / 2}\left(\eta_{\mu \nu} d y^{\mu} d y^{\nu}+f(U) d \tau^{2}\right)+\left(\frac{R}{U}\right)^{3 / 2}\left(\frac{d U^{2}}{f(U)}+U^{2} d \Omega_{4}^{2}\right), \\
& e^{\phi}=g_{s}\left(\frac{U}{R}\right)^{3 / 4}, F_{4}=\frac{N}{V_{4}} \epsilon_{4}, f(U)=1-\frac{U_{K K}^{3}}{U^{3}},
\end{aligned}
$$

donde $d \Omega_{4}^{2}$ y $\epsilon_{4}$ son el elemento de línea invariante ante $S O(5)$, la forma de volumen sobre una 4-esfera unitaria y su volumen es $V_{4}=\frac{8 \pi^{2}}{3}$, mientras que $U_{K K}$ es una constante. Aquí $x^{\mu}$, $\mu=0, \ldots, 3$ y $\tau$ son las direcciones sobre las D4-branas. La última está compactificada en un círculo, mientras que $U$ es la coordenada radial en las direcciones $(5,6,7,8,9)$, transversales a las D4-branas. Para evitar una singularidad cónica en $U=U_{K K}$, el período de $\tau$ debe ser $\delta \tau=\frac{2 \pi}{M_{K K}}=\frac{4 \pi}{3}\left(\frac{R^{3}}{U_{K K}}\right)^{\frac{1}{2}}$. Más detalles de este fondo pueden consultarse en la referencia original [13.

$\mathrm{Al}$ agregar una D6-brana de prueba sobre el fondo 5.2 .8 , la métrica resultante puede escribirse como

$$
d s^{2}=\left(\frac{U}{R}\right)^{3 / 2}\left(\eta_{\mu \nu} d y^{\mu} d y^{\nu}+f(U) d \tau^{2}\right)+\frac{\left(R^{3} U\right)^{\frac{1}{2}}}{\rho(U)^{2}} \overrightarrow{d z} \cdot \overrightarrow{d z}
$$

con $U(\rho)=\left(\rho^{\frac{3}{2}}+\frac{U_{K K}^{3}}{4 \rho^{\frac{3}{2}}}\right)$ y $\vec{z}=\left(z^{5}, \ldots, z^{9}\right)$.

La dinámica de interés para DIS, proceso que estudiaremos en la Segunda Parte de esta Tesis, corresponde al límite $q \gg \Lambda$, donde $\Lambda$ es la escala de energía de confinamiento de la teoría de gauge. Consideraremos entonces la interacción en este límite ultravioleta, región definida por

$$
U_{\text {int }} \sim q^{2} R^{3} \gg U_{0}=\Lambda^{2} R^{3} \equiv U_{K K} .
$$

Podemos tomar entonces el límite de (5.2.10) para $U \gg U_{K K}$, con lo que toma la forma

$$
d s^{2}=\left(\frac{U}{R}\right)^{\frac{3}{2}} \eta_{\mu \nu} d y^{\mu} d y^{\nu}+\left(\frac{R}{U}\right)^{\frac{3}{2}} d U^{2}+\left(\frac{R}{U}\right)^{\frac{3}{2}}\left(d \beta^{2}+\beta^{2} d \Omega_{2}^{2}\right)+\left(\frac{R}{U}\right)^{\frac{3}{2}}\left(d r^{2}+r^{2} d \phi^{2}\right)
$$

con $U^{2}=\beta^{2}+r^{2}$. 


\section{La solución D4D6 $\overline{D 6}$}

Una solución posible para la posición de la D6-brana de prueba en 5.2 .12 es $r(\beta)=0$. Esta es en principio una solución no física (para $U_{K K} \neq 0$ ) si termina en el origen del plano $(U, \tau)$, pues correspondería a una D6-abierta. Sin embargo, esta solución puede extenderse hasta este punto para construir una físicamente aceptable. Una forma de especificar la posición de la D6-brana es fijando las condiciones $\phi=\phi_{0}$ y $\tau=\tau_{0}$. Podemos unir entonces una solución con $\tau=\tau_{0}$ a otra con $\tau=\tau_{0}+\frac{\delta \tau}{2}$ para obtener una solución completamente regular que describa una D6-brana cuya proyección sobre el plano $(U, \tau)$ sea una línea recta que pase por el origen e interseque el borde en dos valores antipódicos de $\tau$. Esto implica que la solución descripta describe la intersección de $N$ D4-branas con una D6-brana y una anti-D6-brane. La razón para la última afirmación es que las orientaciones de las intersecciones con el borde en $\tau=\tau_{0}$ y $\tau=\tau_{0}+\frac{\delta \tau}{2}$, inducidas de una dada orientación de las D6-branas, son opuestas entre sí [13].

Con la elección de esta solución, la métrica inducida sobre la D6-brana es

$$
d s^{2}=\left(\frac{U}{R}\right)^{\frac{3}{2}} \eta_{\mu \nu} d y^{\mu} d y^{\nu}+\left(\frac{R}{U}\right)^{\frac{3}{2}} d U^{2}+R^{\frac{3}{2}} U^{\frac{1}{2}} d \Omega_{2}^{2} .
$$

La introducción del par D6 $\overline{D 6}$ hace que la teoría de gauge dual no preserve ninguna supersimetría. Esta última es la métrica inducida asintótica sobre la que trabajaremos en las Secciones 6.4 y 7.4 .

\subsubsection{Modelo D4D8 $\overline{\mathrm{D} 8}$}

En esta Sección describimos el modelo definido por Sakai y Sugimoto en [14. El mismo se basa en una construcción de D-branas en teoría de cuerdas tipo IIA con $N \gg 1$ D4-branas y $N_{f}$ pares de D8-D8-branas en la aproximación de prueba $N_{f} \ll N$. Este modelo es muy similar al modelo D4D6 $\overline{\mathrm{D} 6}$ descripto en la Sección anterior. La dirección 4 de las D4-branas está compactificada en una circunferencia $S^{1}$, con condiciones de contorno antiperiódicas para los fermiones en la teoría de gauge, lo que rompe todas las supersimetrías $(\mathcal{N}=0)$. Este modelo realiza geométricamente la ruptura de simetría quiral $U\left(N_{f}\right)_{L} \times U\left(N_{f}\right)_{R}$. La coordenada radial $U$, que es transversa a las D4-branas, tiene un valor mínimo $U_{K K}$. El radio de la $S^{1}$ se reduce a cero para $U \rightarrow U_{K K}$. La D8 y la $\overline{\mathrm{D} 8}$ brana se unen en un valor de la coordenada radial $U=U_{0} \geq U_{K K}$. En la D8brana resultante queda entonces solo un factor $U\left(N_{f}\right)$. Esto se interpreta como la representación holográfica de la ruptura espontánea de simetría $U\left(N_{f}\right)_{L} \times U\left(N_{f}\right)_{R} \rightarrow U\left(N_{f}\right)_{V}$.

\section{La solución D4-D8- $\overline{\mathrm{D} 8}$}

Comenzamos con la métrica [14]

$$
\begin{aligned}
& d s^{2}=\left(\frac{U}{R}\right)^{3 / 2}\left(\eta_{\mu \nu} d y^{\mu} d y^{\nu}+f(U) d \tau^{2}\right)+\left(\frac{R}{U}\right)^{3 / 2}\left(\frac{d U^{2}}{f(U)}+U^{2} d \Omega_{4}^{2}\right), \\
& e^{\phi}=g_{s}\left(\frac{U}{R}\right)^{3 / 4}, F_{4}=d C_{3}=\frac{2 \pi N_{c}}{V_{4}} \epsilon_{4}, f(U)=1-\frac{U_{K K}^{3}}{U^{3}},
\end{aligned}
$$

donde $y^{\mu}(\mu=0,1,2,3)$ y $\tau$ son las direcciones de la D4-brana. El elemento de línea en la esfera $S^{4}$ es $d \Omega_{4}^{2}$, la forma de volumen $\epsilon_{4}$, mientras que el volumen de la $S^{4}$ es $V_{4}=8 \pi^{2} / 3$. Los parámetros $R$ y $U_{K K}$ son constantes; $R$ se relaciona con el acoplamiento $g_{s}$ y la longitud fundamental de cuerdas $l_{s}$ mediante $R^{3}=\pi g_{s} N l_{s}^{3}$.

La coordenada $U$ tiene dimensiones de longitud y es la coordenada radial en las direcciones $(5,6,7,8,9)$, transversas a las D4-branas, y es $U \geq U_{K K}$. 
La métrica inducida sobre el volumen de mundo de la D8-brana en este fondo es

$$
d s^{2}=\left(\frac{U}{R}\right)^{3 / 2} \eta_{\mu \nu} d y^{\mu} d y^{\nu}+\left[\left(\frac{U}{R}\right)^{3 / 2} f(U)+\left(\frac{R}{U}\right)^{3 / 2} \frac{U^{\prime 2}}{f(U)}\right] d \tau^{2}+\left(\frac{R}{U}\right)^{3 / 2} U^{2} d \Omega_{4}^{2},
$$

con $U^{\prime}=\frac{d}{d \tau} U$. La acción de la D8-brana es

$$
S_{D 8} \propto \int d^{4} y d \tau \epsilon_{4} e^{-\phi} \sqrt{\left|\operatorname{det}\left(g_{D 8}\right)\right|} \propto \int d^{4} y d \tau U^{4} \sqrt{f(U)+\left(\frac{R}{U}\right)^{3} \frac{U^{\prime 2}}{f(U)}} .
$$

Podemos despejar $\tau$ en función de $U$, con lo que (5.2.16) se escribe

$$
d s^{2}=\left(\frac{U}{R}\right)^{3 / 2} \eta_{\mu \nu} d y^{\mu} d y^{\nu}+\left(\frac{R}{U}\right)^{3 / 2} \frac{U^{8}}{\left(U^{8} f(U)-U_{0}^{8} f\left(U_{0}\right)\right)} d U^{2}+\left(\frac{R}{U}\right)^{3 / 2} U^{2} d \Omega_{4}^{2}
$$

La característica fundamental de la geometría (5.2.18), como del resto de los modelos considerados en este Capítulo, es el corrimiento al rojo gravitacional. El momento $p_{\mu}=-i \partial_{\mu}$ en la teoría de gauge es visto por un observador inercial en la $\mathrm{D} p$-brana como

$$
\tilde{p}_{\mu}=\frac{1}{\sqrt{\left|g_{t t}\right|}} p_{\mu} .
$$

La escala característica de energía en la $\mathrm{D} p$-brana es $R^{-1}$, y por esto la escala de energía en cuatro dimensiones es

$$
E \sim\left(\frac{U^{3}}{R^{7}}\right)^{\frac{1}{4}}
$$

La dinámica de interés para el proceso a estudiar de DIS corresponde al límite $q \gg \Lambda$, donde $\Lambda$ es la escala de energía de confinamiento de la teoría de gauge. Con esto consideraremos la interacción en el límite UV, región dada por

$$
U_{\text {int }} \sim q^{2} R^{3} \gg U_{0}=\Lambda^{2} R^{3} \equiv U_{K K} .
$$

En esta región, la métrica $(5.2 .18)$ puede escribirse

$$
d s^{2}=\left(\frac{U}{R}\right)^{\frac{3}{2}} \eta_{\mu \nu} d y^{\mu} d y^{\nu}+\left(\frac{R}{U}\right)^{\frac{3}{2}} d U^{2}+R^{\frac{3}{2}} U^{\frac{1}{2}} d \Omega_{2}^{2} .
$$

La similitud con 5.2 .13 es notoria. La única diferencia consiste en que las coordenadas $z^{8}$ y $z^{9}$ no pertenecen a la $\mathrm{D} p$-brana de prueba en este caso. 


\section{Parte II}

\section{Contribuciones originales}




\section{Capítulo 6}

\section{Estudio del caso $x \lesssim 1$ : régimen de supergravedad}

Expondremos en este Capítulo el estudio de las funciones de estructura para el régimen cinemático $x \lesssim 1$ (estrictamente $\frac{1}{\sqrt{\lambda}} \ll x<1$ región $\mathbf{A}$ ) para mesones escalares y vectoriales (tanto con uno como con varios sabores) provenientes del modelo D3D7 [12] y luego lo extenderemos a los modelos $\mathrm{D} 4 \mathrm{D} 8 \overline{\mathrm{D} 8}$ [14 y $\mathrm{D} 4 \mathrm{D} 6 \overline{\mathrm{D} 6}$ [13. La idea principal se basa en el método descripto en [5] y que explicaremos la Sección 6.1 de la presente Tesis. Los resultados aquí reproducidos fueron publicados originalmente en nuestros trabajos [1] y [2].

Queremos responder a la pregunta de si es posible extraer las ocho funciones de estructura para hadrones dinámicos holográficos [55] y qué puede decirse sobre la dependencia en $x$ de estas funciones de estructura. Podemos encarar estas preguntas en el límite planar y en el acoplamiento fuerte. No se conocen modelos holográficos duales para bariones dinámicos, por lo que consideraremos mesones, tanto escalares como vectoriales polarizados. Esto es interesante por varias razones. Una es entender la estructura de los mesones duales holográficos. Otra es que estamos interesados en buscar propiedades generales, a saber, propiedades que bien no dependan del modelo holográfico dual particular, o bien dependan de este en una manera en la que podamos inferir lo que pasará para las funciones de estructura de mesones de QCD en el límite de $N$ grande.

En [1] comenzamos con este programa de investigación para mesones dinámicos holográficos escalares y polarizados con un sabor, considerando dos modelos diferentes de $\mathrm{D} p$-branas de sabor, uno en la teoría de supercuerdas tipo IIB y otro en la tipo IIA. Luego, en 2] generalizamos esta investigación al caso de varios sabores, lo que es una generalización no trivial, obteniendo también los lagrangianos correspondientes al primer orden subdominante en las expansiones $\frac{1}{N} \mathrm{y}$ $\frac{N_{f}}{N}$, así como extendiéndolo a un modelo más general de $\mathrm{D} p$-branas, lo que corresponde a distintas teorías de gauge confinantes. En particular, hemos estudiado el modelo de D3D7-branas, dual a una teoría supersimétrica de Yang-Mills $\mathcal{N}=2$ con quarks en la representación fundamental [12], y también en teorías de gauge no supersimétricas duales al modelo de $\mathrm{D} 4 \mathrm{D} 8 \overline{\mathrm{D}} 8$-branas de Sakai y Sugimoto [14 y el modelo de D4D6 $\overline{\mathrm{D} 6}$-branas 13 . Para estas tres teorías de gauge confinantes hemos obtenido las funciones de estructura de mesones escalares y vectoriales polarizados en el límite de supergravedad ( i.e. en la región $\mathbf{A}$, con $\frac{1}{\sqrt{\lambda}} \ll x<1$ ). Un esquema de la descripción holográfica para la dispersión Compton en esta región paramétrica (SUGRA pura) puede verse en la Figura 6.1 .

Hemos encontrado varios resultados interesantes. Por un lado, obtuvimos nuevas relaciones entre varias de las ocho funciones de estructura para los mesones vectoriales polarizados. Por otro lado, encontramos que estas relaciones son independientes del modelo: podrían presentar 
una estructura universal para mesones holográficos escalares y vectoriales. La razón de este comportamiento es el hecho de que la dinámica de los modelos duales holográficos con D $p$-branas de prueba de sabor está controlada por la acción de Dirac-Born-Infeld (DBI). A pesar de que para cada modelo la acción de DBI varía su dimensión, así como la estructura de los campos de gauge y la métrica inducida, da lugar a relaciones del tipo Callan-Gross [63, 64, 65, 66] independientes del modelo de D $p$-branas considerado para esta región, como por ejemplo $F_{2}=2 F_{1}$. Si bien la relación usual para $x$ pequeño en el caso de QCD es $F_{2}=2 x F_{1}$, justificaremos con argumentos físicos esta discrepancia en este Capítulo, y descubriremos una relación muy similar a la usual entre estas funciones de estructura cuando estudiemos el caso de $x$ pequeño desde el punto de vista holográfico en el próximo Capítulo.

\section{1. $\quad$ DIS desde glueballs}

Si bien el tratamiento del DIS de glueballs fue estudiado en [5] y por lo tanto no es una contribución original de esta Tesis, la incluimos aquí con el fín de dar un sentido autocontenido a la discusión que sigue a partir de la Sección 6.2. Es a partir de esta que el contenido consiste de aportes originales, correspondientes a los trabajos [1, 2] a lo largo de este Capítulo.

\section{Generalidades}

En esta Sección calcularemos las funciones de estructura $F_{1}$ y $F_{2}$ en el marco de la teoría $\mathcal{N}=1^{*}$ SYM, siguiendo el procedimiento de Polchinski y Strassler [5]. En las próximas Secciones de este Capítulo utilizaremos gran parte de esta presentación para aplicarlo al caso de interés con D-branas de sabor.

Dada una teoría de gauge conforme, el modelo dual de teoría de cuerdas está en un espacio de la forma $A d S_{5} \times W$, donde $W$ puede ser $S^{5}$ u otro espacio de Einstein compacto de cinco dimensiones. La métrica más general es 5.1.1

$$
d s^{2}=\frac{r^{2}}{R^{2}} \eta_{\mu \nu} d y^{\mu} d y^{\nu}+\frac{R^{2}}{r^{2}} d r^{2}+R^{2} \widehat{d s}_{W}^{2}
$$

donde $R=\left(4 \pi g_{s} N \alpha^{2}\right)^{1 / 4}$ es el radio del $A d S_{5}$, así como de la esfera $S^{5}$. Las coordenadas $y^{\mu}$ serán las que identificaremos con la teoría de gauge, mientras que $r$ será la coordenada radial holográfica, y las coordenadas dentro del espacio $W$ serán denotadas genéricamente con $\Omega$.

Es importante notar aquí el papel del corrimiento al rojo gravitatorio (gravitational redshift) debido al factor de deformación (warp factor) $g_{t t}=\frac{r^{2}}{R^{2}}$ frente a la parte tetradimensional de la métrica. El momento de esta parte de la métrica, $p_{\mu}=-i \partial_{\mu}$ con $\mu=0, \ldots, 3$, vistos por un observador inercial en diez dimensiones es

$$
\tilde{p}_{\mu}=\frac{R}{r} p_{\mu} .
$$

La escala de energía característica en diez dimensiones es, por análisis dimensional, $\tilde{E}=R^{-1}$. Por ende, la energía en cuatro dimensiones es $E \sim \frac{r}{R^{2}}$, de lo que resulta $E(r=0) \rightarrow 0$ en el horizonte y $E(r=\infty) \rightarrow \infty$ en el borde del $A d S_{5}$.

Si la teoría es confinante la métrica será (5.1.1) solamente de manera asintótica para grandes valores de $r$, mientras que será diferente para radios del orden de

$$
r_{0} \equiv \Lambda R^{2},
$$

donde $\Lambda$ es la masa del estado de glueball más liviano y definirá una escala característica de energía (mass gap). Esto protege al warp factor de anularse. 
Trabajaremos siempre con interacciones que sucedan para grandes valores de $q$, lo que implica grandes valores de $r$ [

$$
q_{\text {int }} \gg \Lambda \quad \Rightarrow \quad r_{\text {int }}=q_{\text {int }} R^{2} \gg \Lambda R^{2}=r_{0},
$$

y por lo tanto no nos importará la física en regiones cercanas a este cutoff $r_{0}$. Esta teoría resultante será similar al límite planar de QCD, asintóticamente conforme en el UV, con confinamiento de color y mass gap en el IR. Sin embargo, a diferencia de QCD, puede ser fuertemente acoplada en el UV.

Todo lo dicho hasta aquí vale para un gran número de modelos, desde $\mathcal{N}=1^{*} \mathrm{SYM}$ [11], el modelo D3D7 12 y teorías que tienen un flujo del grupo de renormalización holográfico como las de las referencias [57, 58, 62. Un punto fundamental es que el modelo en cuestión posea una simetría global que contenga a $U(1)$ como un subgrupo, lo que permitirá realizar la interacción electromagnética en el modelo dual $\left.\right|^{2}$

\section{Cálculo del tensor hadrónico}

Como dijimos en el Capítulo 4, nuestro objetivo es calcular el elemento de martiz ${ }^{3}$

$$
\mathrm{T}^{\mu \nu}=i\left\langle P, \mathcal{Q}\left|T\left(\tilde{J}^{\mu}(q) J^{\nu}(0)\right)\right| P, \mathcal{Q}\right\rangle
$$

cuya parte imaginaria es

$$
\begin{aligned}
\operatorname{Im} T^{\mu \nu} & =\pi \sum_{P_{X}, X}\left\langle P, \mathcal{Q}\left|J^{\nu}(0)\right| P_{X}, X\right\rangle\left\langle P_{X}, X\left|\tilde{J}^{\mu}(q)\right| P, \mathcal{Q}\right\rangle \\
& =2 \pi^{2} \sum_{X} \delta\left(M_{X}^{2}+[P+q]^{2}\right)\left\langle P, \mathcal{Q}\left|J^{\nu}(0)\right| P+q, X\right\rangle\left\langle P+q, X\left|J^{\mu}(0)\right| P, \mathcal{Q}\right\rangle
\end{aligned}
$$

Tanto en esta Sección como en el resto de la Tesis utilizaremos la siguiente notación:

- Índices $M, N, \ldots=0, \ldots, 9$ representan el espacio completo 10-dimensional.

- Índices $a, b, \ldots=0, \ldots, p$ representan el volumen de mundo de una $\mathrm{D} p$-brana. 4

- Índices $m, n, \ldots=0, \ldots 4$ representan el espacio $A d S_{5} \cdot{ }^{5}$ Estos se separan en:

- Índices $\mu, \nu, \ldots=0, \ldots 3$ correspondientes al espacio de Minkowski

- Índice $r=4$ correspondiente a la coordenada radial.

- Índices $i, j, \ldots=5, \ldots, p$ representan el espacio $W[6$

\footnotetext{
${ }^{1}$ Justificaremos esta implicación luego de la ecuación 6.1.18.

${ }^{2}$ Los modelos $D 4 D 8 \overline{D 8}$ [14 y $D 4 D 6 \overline{D 6}$ [13] no cumplen estrictamente con lo que describimos anteriormente por no ser conformes en el límite UV. Igualmente serán analizados con un formalismo muy similar al presente en los Capítulos 6 y 7 pues cumplen con la hipótesis de poseer el subgrupo de simetría global $U(1)$.

${ }^{3}$ Notamos $\tilde{J}^{\mu}$ a la transformada de Fourier de $J^{\mu}$.

${ }^{4}$ Estos índices aparecerán en las siguientes Secciones de este Capítulo, cuando consideremos D $p$-branas de sabor. En el caso sin branas de sabor consideramos $p=9$ y estos índices coinciden con $M, N, \ldots=$ $0, \ldots, 9$.

${ }^{5} \mathrm{O}$ espacio formado por las primeras cinco coordenadas, que en los modelos [14, 13, no es $A d S_{5}$.

${ }^{6}$ Coincide con $S^{5}$ solamente en el presente caso, sin branas de sabor.
} 
Notemos que cuando contraigamos cuadrivectores definidos en el espacio de Minkowski, como por ejemplo los momentos $P_{\mu}, q_{\mu}$, la polarización $n_{\mu}$ y las corrientes $J_{\mu}$ lo haremos con la métrica plana y se notarán con un punto $\cdot$, es decir

$$
P \cdot q \equiv P_{\mu} q^{\mu}=\eta_{\mu \nu} P^{\mu} q^{\nu},
$$

mientras que las contracciones entre vectores en 10,5 o $(p+1)$ dimensiones serán hechas con la métrica completa, a saber

$$
\begin{aligned}
X^{M} Y_{M} & \equiv g_{M N} X^{M} Y^{N} \\
& =X^{\tilde{\mu}} Y_{\tilde{\mu}}+X^{r} Y_{r}+X^{i} Y_{i} \\
& =g_{\tilde{\mu} \tilde{\nu}} X^{\tilde{\mu}} Y^{\tilde{\nu}}+g_{r r} X^{r} Y^{r}+g_{i j} X^{i} Y^{j} \\
& =\frac{r^{2}}{R^{2}} \eta_{\mu \nu} X^{\mu} Y^{\nu}+\frac{R^{2}}{r^{2}} X^{r} Y^{r}+R^{2} \hat{g}_{i j} X^{i} Y^{i}
\end{aligned}
$$

En el límite planar de la teoría de gauge contribuyen solamente estados de un hadrón. Para el canal $s$ tendremos $\square^{7}$

$$
s=-(P+q)^{2}=q^{2}\left[\frac{1}{x}-1-t\right] \simeq q^{2}\left[\frac{1}{x}-1\right],
$$

donde utilizamos que $|t|=-\frac{P^{2}}{q^{2}} \ll 1$. La escala correspondiente en diez dimensiones, considerando el corrimiento al rojo gravitatorio, es

$$
\tilde{s}=-g^{M N} P_{X, M} P_{X, N} \leq-g^{\mu \nu}(P+q)_{\mu}(P+q)_{\nu} \sim \frac{R^{2}}{r_{i n t}^{2}} q^{2}\left(\frac{1}{x}-1\right)=\frac{\left(\frac{1}{x}-1\right)}{\alpha^{\prime}\left(4 \pi g_{s} N\right)^{1 / 2}},
$$

donde $P_{X}=P+q$ es el momento del hadrón final. El parámetro de 't Hooft aparece en el denominador, por lo que si $\lambda^{-1 / 2}=\left(g_{s} N\right)^{-1 / 2} \ll x<1$, tenemos $\alpha^{\prime} \tilde{s} \ll 1$. Entonces, para $x \lesssim 1$ solo se producen estados de cuerdas no masivos y por ende estamos considerando un proceso puramente de supergravedad. Este es el régimen que utilizaremos en esta Sección y durante todo este Capítulo.

Estudiemos ahora la forma en que se realiza el DIS desde el punto de vista de la teoría de supergravedad, en el interior del espacio $A d S$. La idea es calcular el elemento de matriz (6.1.4), que es una función de dos puntos correspondiente a dos corrientes electromagnéticas dentro del hadrón. Al utilizar la dualidad $A d S / C F T$, el operador de corriente insertado en el borde del $A d S$ induce una perturbación en la condición de contorno de un campo de gauge en el interior. Esta perturbación excita un modo no normalizable, que se propagará hacia el interior [25, 24]. Este campo de gauge del interior es un campo de Kaluza-Klein de una perturbación de la métrica. El grupo de isometría de la variedad $W$ corresponde, en la teoría de gauge, a una simetría globa 8 . Esta será la isometría de una esfera $S^{5}$ (i.e. $\left.S O(6)\right)$ en el caso de Polchinski y Strassler, y la de una esfera $S^{3}, S^{4}$ y $S^{2}$ respectivamente en los modelos $D 3 D 7, D 4 D 8 \overline{D 8}$ y $D 4 D 6 \overline{D 6}$. En todos los casos, estas isometrías poseen un subgrupo de simetría global $U(1)$, el cual tiene asociada una corriente $J_{\mu}$, la cual se promueve a ser una corriente $J_{\mu E M}$ asociada a una simetría de gauge $U(1)$ en la teoría de campos dual, cuyo campo de gauge llamaremos $A_{m}$. La mencionada

${ }^{7}$ En un proceso de colisión $k_{1}+k_{2} \rightarrow-k_{3}-k_{4}$ definimos las variables de Mandelstam $s=-\left(k_{1}+k_{2}\right)^{2}$, $t=-\left(k_{1}+k_{4}\right)^{2}$ y $u=-\left(k_{1}+k_{3}\right)^{2}$.

${ }^{8}$ Esta será una simetría $R$ en el caso de Polchinski y Strassler y en el D3D7. Como anticipamos en 6.1.3), estamos trabajando para valores de $r \gg r_{0}$, donde el espacio es asintóticamente $A d S_{5} \times W$. 
simetría global $U(1)$ es generada por el vector de Killing $v_{i}$. Esta corriente excita un modo no normalizable del campo de gauge de Kaluza-Klein de la form $2^{9}$

$$
\delta g_{m i}=A_{m}(y, r) v_{i}(\Omega),
$$

con condición de contorno

$$
\lim _{r \rightarrow \infty} A_{\mu}(y, r)=\left.A_{\mu}(y)\right|_{4 \mathrm{~d}}=n_{\mu} e^{i q \cdot y},
$$

es decir, el límite de este modo no normalizable coincide con el campo de gauge en la teoría de cuatro dimensiones. Este campo de gauge corresponderá a la inserción del operador $n_{\mu} \tilde{J}^{\mu}(q) 10$.

El blanco del DIS será un glueball, que será representado en la teoría de supergravedad por un dilatón, factorizado en la forma

$$
\Phi=e^{i P \cdot y} \psi(r, \Omega)=e^{i P \cdot y} f(r) Y(\Omega) .
$$

La función $\psi(r, \Omega)$ es un modo normalizable en el espacio $A d S_{5} \times W$. Tomaremos tanto el hadrón inicial como el final como cuerdas cerradas en su estado fundamental, no masivas en 10 dimensiones. En particular, tomaremos el dilatón, de espín cero, que será considerado como el dual al glueball de la teoría de gauge $\mathcal{N}=1^{*}$ del borde del $A d S_{5}$. Las corrientes (operadores de la teoría de gauge dual del borde) corresponden a perturbaciones de las condiciones de contorno en el borde $(r=\infty)$, que excitan modos no normalizables en el interior (bulk) del espacio.

El proceso se desarrolla de la siguiente manera: el dilatón entrante $\Phi_{i}$ se acopla con el campo de gauge del interior $A_{\mu}$, inducido por la corriente insertada en el borde, y a un estado dilatónico intermedio $\Phi_{X}$, como se muestra en la Figura 6.1. Este último se propaga por el interior hasta acoplarse con un dilatón saliente $\Phi_{f}$ (estado final del dilatón) y con el campo de gauge $A_{\nu}$, proveniente de la inserción de una segunda corriente en el borde. Este diagrama de Witten representa la versión dual del teorema óptico [5] para el proceso de DIS que se muestra esquemáticamente en el plano correspondiente al borde. Se muestran también los leptones (indicados con $l$ ) y su acoplamiento a los fotones virtuales, indicados con líneas de guiones. Estos fotones luego se acoplan a las corrientes dentro de los hadrones, que se indican con líneas sólidas en el plano del borde, y están rotulados por sus momentos $P_{i}, P_{X}$ y $P_{f}$. En este régimen cinemático el leptón es dispersado por un hadrón entero, que se excita pero no se fragmenta.

\section{Cálculo de los campos intervinientes}

Ahora tenemos que encontrar las soluciones tanto para el campo de gauge $A_{m}$ como para los dilatones $\Phi_{i}, \Phi_{X}$ y $\Phi_{f}$. Comenzamos por el campo de gauge $A_{m}$ : partiendo de la ecuación de Maxwell en 5 dimensiones

$$
D_{m} F^{m n}=0
$$

resulta

$$
\begin{aligned}
\square A_{r}-\partial_{r}(\partial \cdot A) & =0, \\
\square A^{\mu}-\partial^{\mu}(\partial \cdot A)+\left(\frac{r}{R}\right) \partial_{r}\left[\left(\frac{r}{R}\right)^{3}\left(\partial_{r} A^{\mu}-\partial^{\mu} A_{r}\right)\right] & =0,
\end{aligned}
$$

${ }^{9}$ La ecuación 6.1.11 es esquemática. La expresión correcta, simétrica, es

$$
\delta g_{a b}=\frac{1}{2}\left[A_{a}(y, r) v_{b}(\Omega)+A_{b}(y, r) v_{a}(\Omega)\right],
$$

con $A_{a}(y, r)=\left(A_{m}(y, r), 0\right)$ y $v_{a}(\Omega)=\left(0, v_{i}(\Omega)\right)$.

${ }^{10}$ Pues $\int d^{4} y A_{\mu}(y, \infty) J^{\mu}(y)=n_{\mu} \int d^{4} y e^{i q \cdot y} J^{\mu}(y)=n_{\mu} \tilde{J}^{\mu}(q)$ 


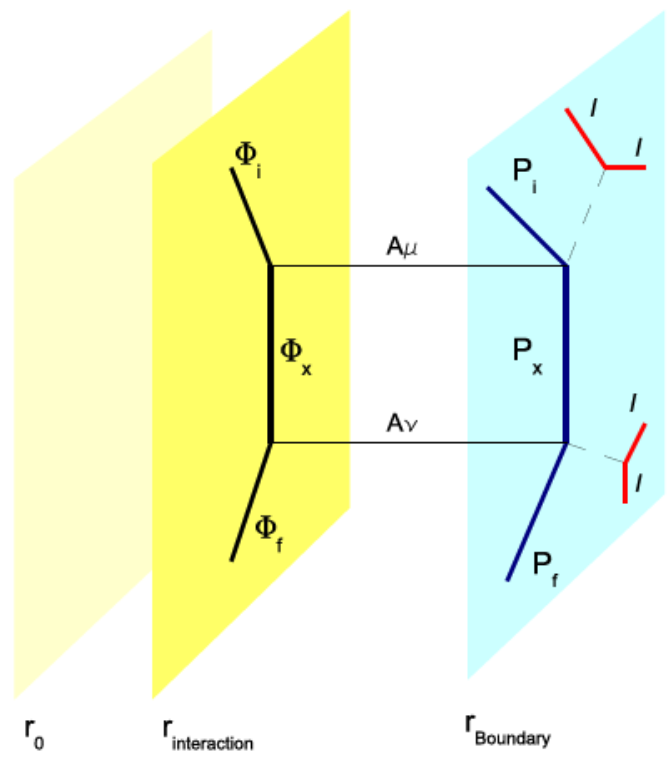

Figura 6.1: Representación esquemática de la prescripción holográfica para el cálculo del tensor hadrónico. La interacción ocurre en el interior en el radio $r_{\text {interaction. También se }}$ muestran cortes representando el cutoff IR $r_{0}$ y la descripción del proceso holográfico en el borde. Figura tomada de nuestro paper [1]. 
Proponiendo ahora una solución de la forma ${ }^{11}$

$$
\begin{aligned}
& A^{\mu}(y, r)=n^{\mu} e^{i q \cdot y} g(r), \\
& A_{r}(y, r)=e^{i q \cdot y} h(r),
\end{aligned}
$$

y con las condiciones de contorno 6.1 .12 y

$$
\lim _{r \rightarrow \infty} A_{r}(y, r)=0
$$

se obtient 12

$$
\begin{aligned}
A^{\mu} & =n^{\mu} e^{i q \cdot y}\left(\frac{q R^{2}}{r}\right) K_{1}\left(\frac{q R^{2}}{r}\right), \\
A_{r} & =-i(q \cdot n) R e^{i q \cdot y}\left(\frac{R}{r}\right)^{3} K_{0}\left(\frac{q R^{2}}{r}\right), \\
& =-\frac{i}{q^{2}} \eta^{\mu \nu} q_{\mu} \partial_{r} A_{\nu},
\end{aligned}
$$

donde definimos $q \equiv \sqrt{q^{2}}$.

Vemos aquí la justificación de la ecuación (6.1.3): del comportamiento exponencial de las funciones de Bessel modificadas se desprende que para $r<q R^{2}$ el campo $A_{m}$ decae rápidamente. Es decir, para el rango cinemático en el que estamos interesados, $q \gg \Lambda$, podemos considerar que la interacción sucede solamente en la zona $r_{\text {int }} \sim q_{\text {int }} R^{2}$.

Pasemos ahora al dilatón. Para el hadrón inicial y final, que estarán definidos en la región de interacción $r_{\text {int }} \gg r_{0}$, podremos utilizar el comportamiento dominante en esta región. Esto puede verse más en detalle en la referencia [67],

$$
\Phi_{\mathrm{i}}=\Phi_{\mathrm{f}}^{*} \equiv\langle 0|\Phi| P, \mathcal{Q}\rangle=e^{i P \cdot y} f(r) Y(\Omega), \quad f(r) \propto r^{-\Delta} .
$$

En una teoría conforme los autoestados de carga tendrán esta forma donde $\Delta$ es la dimensión conforme del estado, así como del operador local que crea este estado desde el vacío. En el presente caso, la dinámica no conforme para valores pequeños de $r$ causa una mezcla entre estos términos, mientras que para valores grandes de $r$ el término dominante es el de menor $\Delta$. Para un campo escalar canónicamente normalizado, la cuantización canónica resulta 13

$$
\int d r d^{5} \Omega e^{2 A} \sqrt{g_{\perp}}|\psi(r, \Omega)|^{2}=1
$$

donde

$$
d s^{2}=e^{2 A(r, \Omega)} \eta_{\mu \nu} d y^{\mu} d y^{\nu}+d s_{\perp}^{2} .
$$

La integral de normalización está dominada por la región IR $r \sim r_{0}$, donde $e^{2 A} \sqrt{g_{\perp}} \sim r_{0} R^{4}$. De aquí vemos que $\psi\left(r_{0}, \Omega\right) \sim 1 / R^{2} r_{0} \mathrm{y}$

$$
f(r)=\frac{c_{\mathrm{i}}}{R^{4} \Lambda}\left(\frac{r}{r_{0}}\right)^{-\Delta}
$$

donde $c_{\mathrm{i}}$ es una constante adimensional. Hemos definido la función de onda angular de manera que la normalización resulte adimensional,

$$
\int d^{5} \Omega \sqrt{\hat{g}_{W}}|Y(\Omega)|^{2}=1,
$$

\footnotetext{
${ }^{11}$ Esta propuesta ya implica la elección de un gauge tipo Lorenz.

${ }^{12}$ En la referencia [5] no aparece el signo (-) en la segunda ecuación.

${ }^{13}$ En el apéndice de [67] se encuentra el detalle de la normalización utilizada.
} 
donde $\left(\hat{g}_{W}\right)_{a b}$ es la parte adimensional de la métrica sobre $W$. Los detalles de la ruptura de la simetría conforme solo cambiarán el valor de $c_{\mathrm{i}}{ }^{14}$

En el interior del espacio $A d S$ la función de onda para el dilatón $\Phi_{X}$ satisfará la ecuación de Klein-Gordon en cinco dimensiones para un escalar de masa $M^{2}=\frac{\Delta(\Delta-4)}{R^{2}}$, cuya solución es

$$
\Phi_{X}=e^{i(P+q) \cdot y} \frac{C}{r^{2}} J_{\Delta-2}\left(\frac{s^{1 / 2} R^{2}}{r}\right) Y(\Omega),
$$

donde $J_{\Delta-2}$ es la función de Bessel de primera especie de orden $\Delta-2$ y $s=-(P+q)^{2}=M_{X}^{2}$ es el cuadrado de la masa del estado intermedio, mientras que $C$ es una constante.

Notemos que $X$ será una excitación de gran energía, dado que su masa crece con $q^{2}$ (ver 6.1.8). Con esto, el radio de la función de Bessel modificada es $s^{1 / 2} R^{2} \gg r_{0}$, por lo que debemos utilizar la forma completa de la función de Bessel y no su forma asintótica (6.1.19) que utilizamos para los estados inicial y final. La normalización 6.1.20 está también ahora dominada por $r \sim r_{0}$, resultando $C=c_{X} s^{1 / 4} \Lambda^{1 / 2}$ con $c_{X}$ una constante adimensional.

Con esto, la forma asintótica de la función de onda para el dilatón entrante y saliente 6.1.22 equivale a tomar el límite para $r \gg r_{0}$ de (6.1.24).

\section{Acción de interacción}

El término de interacción para la acción de supergravedad que se obtiene insertando la perturbación de la métrica 6.1.11 es

$$
S=\int d^{10} x \sqrt{-g} A^{m} v^{j} \partial_{m} \Phi \partial_{j} \Phi .
$$

El dilatón que tomaremos es un autoestado de carga $\mathcal{Q}$, autovalor asociado a la simetría $U(1)$ en la dirección del vector de Killing $v^{j}$,

$$
v^{j} \partial_{j} Y(\Omega)=i \mathcal{Q} Y(\Omega) .
$$

Si evaluamos esta acción en el elemento de matríz de la interacción obtenemos

$$
\begin{aligned}
S_{\text {int }} & =\langle i n|S| X\rangle \\
& =i \mathcal{Q} \int d^{10} x \sqrt{-g} A^{m}\left(\Phi_{i} \partial_{m} \Phi_{X}^{*}-\Phi_{X}^{*} \partial_{m} \Phi_{i}\right),
\end{aligned}
$$

donde $\Phi_{i}$ denota el estado $I N / O U T$ y $\Phi_{X}$ el estado intermedio.

Aplicaremos ahora la prescripción holográfica. Lo que queremos calcular del lado de la teoría de gauge es el elemento de matriz $\left\langle P_{X}, X\left|J^{\mu}(0)\right| P, \mathcal{Q}\right\rangle$. La idea será identificar la funcional generatriz de las funciones de Green conexas de la teoría de gauge con el mínimo $K$ de la acción de supergravedad $S$, con condiciones de contorno en $r=\infty$, según se muestra en [25]

$$
W\left[A_{m}\left(x^{\mu}\right)\right]=K\left[A_{m}\left(x^{\mu}\right)\right]
$$

donde $W\left[A_{m}\left(x^{\mu}\right)\right]$ es la función generatriz de las funciones de Green conexas de la teoría de gauge y $K\left[A_{m}\left(x^{\mu}\right)\right]$ es el mínimo valor de la acción de supergravedad $S$ en el espacio $A d S$ fijadas las condiciones de contorno en $r \rightarrow \infty$, es decir

$$
\left.K\left[A_{m}\left(x^{\mu}\right)\right] \equiv \min S\left[A_{m}\left(x^{\mu} \cdot r\right)\right]\right|_{b c} .
$$

\footnotetext{
${ }^{14}$ En las Secciones 6.2 y 6.3 estudiaremos este mismo problema en el fondo de D3D7-branas. Allí la normalización correcta será $f(r)=\frac{c_{i}}{R^{3} \Lambda}\left(\frac{r}{r_{0}}\right)^{-\Delta}$. En la Sección 6.4 lo haremos en el caso general de un fondo con una $\mathrm{D} p$-brana de prueba, donde la normalización será $f(r)=\frac{c_{\mathrm{i}}}{R^{\frac{p-1}{2}} \Lambda}\left(\frac{r}{r_{0}}\right)^{-\Delta}$.
} 
Vale recordar que en la convención de [25] esta función generatriz se calcula orden a orden. Estamos interesados en la función de correlación de un punto, la que calcularemos del lado de supergravedad. Para ello, partimos de una expresión para la acción $K$ en términos del valor de borde de $A_{m}$, es decir $A_{r}(y, \infty)=0$ y $A_{\mu}(y, \infty)=n_{\mu} e^{i q . y}$,

$$
\begin{aligned}
K\left[A_{\mu}(y, \infty)\right]= & i \mathcal{Q} \int d^{10} x \sqrt{-g} A_{m}\left(\Phi_{i} \partial^{m} \Phi_{X}^{*}-\Phi_{X}^{*} \partial^{m} \Phi_{i}\right) \\
= & i \mathcal{Q} \int d^{10} x \sqrt{-g} A_{\mu}\left(\Phi_{i} \partial^{\mu} \Phi_{X}^{*}-\Phi_{X}^{*} \partial^{\mu} \Phi_{i}\right) \\
& +i \mathcal{Q} \int d^{10} x \sqrt{-g} A_{r}\left(\Phi_{i} \partial^{r} \Phi_{X}^{*}-\Phi_{X}^{*} \partial^{r} \Phi_{i}\right) \\
= & i \mathcal{Q} \int d^{10} x \sqrt{-g} A_{\mu}(y, \infty) g(r)\left(\Phi_{i} \partial^{\mu} \Phi_{X}^{*}-\Phi_{X}^{*} \partial^{\mu} \Phi_{i}\right) \\
& +i \mathcal{Q} \int d^{10} x \sqrt{-g} n^{\nu} A_{\nu}(y, \infty) h(r)\left(\Phi_{i} \partial^{r} \Phi_{X}^{*}-\Phi_{X}^{*} \partial^{r} \Phi_{i}\right)
\end{aligned}
$$

donde

$$
g(r)=\frac{q R^{2}}{r} K_{1}\left(\frac{q R^{2}}{r}\right) \quad ; \quad h(r)=i q \cdot n \frac{R^{4}}{r^{3}} K_{0}\left(\frac{q R^{2}}{r}\right),
$$

son las funciones definidas en la propuesta (6.1.16) y utilizamos $n_{\mu} n^{\mu}=1$. Notar que las formas asintóticas de estas para $r \gg q R^{2}$ son

$$
g(r) \simeq 1 \quad ; \quad h(r) \simeq i(q \cdot n) \frac{R^{4}}{r^{3}} \ln \left(\frac{q R^{2}}{2 r}\right) \rightarrow 0,
$$

Según la correspondencia $A d S / C F T$, en [25], $K\left[A_{\mu}(y, \infty)\right]$ corresponde a la funcional generatriz para funciones de correlación del operador dual $J_{\mu}^{e m}$ en la teoría de campos del borde del $A d S_{5}$, por lo que

$$
\begin{aligned}
\left\langle J^{\mu}(y)\right\rangle= & \left.\frac{\delta}{\delta A_{\mu}(y, \infty)} K\left[A_{\mu}(y, \infty)\right]\right|_{A_{\mu}(y, \infty)=0} \\
= & i \mathcal{Q} \int d r d \Omega \sqrt{-g} g(r)\left(\Phi_{i} \partial^{\mu} \Phi_{X}^{*}-\Phi_{X}^{*} \partial^{\mu} \Phi_{i}\right) \\
& +i \mathcal{Q} \int d r d \Omega \sqrt{-g} n^{\mu} h(r)\left(\Phi_{i} \partial^{r} \Phi_{X}^{*}-\Phi_{X}^{*} \partial^{r} \Phi_{i}\right) .
\end{aligned}
$$

Es fácil ver que la transformada de Fourier $n_{\mu}\left\langle\tilde{J}^{\mu}(q)\right\rangle$ resulta

$$
\begin{aligned}
n_{\mu}\left\langle\tilde{J}^{\mu}(q)\right\rangle & =n_{\mu} \int d^{4} y\left\langle J^{\mu}(y)\right\rangle e^{i q \cdot y} \\
& =i \mathcal{Q} \int d^{10} x \sqrt{-g} A_{m}\left(\Phi_{i} \partial^{m} \Phi_{X}^{*}-\Phi_{X}^{*} \partial^{m} \Phi_{i}\right) \\
& =S_{\text {int }}
\end{aligned}
$$

Es decir, hemos demostrado la prescripción holográfica

$$
n_{\mu}\left\langle P_{X}, X\left|\tilde{J}^{\mu}(q)\right| P, \mathcal{Q}\right\rangle=S_{\text {int }}
$$

utilizada en [5]. Continuando con el cálculo, vemos que este se simplifica separándolo en dos integrales

$$
\begin{aligned}
n_{\mu}\left\langle\tilde{J}^{\mu}(q)\right\rangle= & i \mathcal{Q} \int d^{10} x \sqrt{-g} A_{\mu}\left(\phi_{i} \partial^{\mu} \phi_{X}^{*}-\phi_{X}^{*} \partial^{\mu} \phi_{i}\right) \\
& +i \mathcal{Q} \int d^{10} x \sqrt{-g} A_{r}\left(\phi_{i} \partial^{r} \phi_{X}^{*}-\phi_{X}^{*} \partial^{r} \phi_{i}\right) \\
\equiv & I_{1}+I_{2} .
\end{aligned}
$$


Para el cálculo de $I_{1}$ e $I_{2}$ usaremos las normalizaciones

$$
\begin{aligned}
& \int d \Omega \sqrt{\tilde{g}_{W}}|Y(\Omega)|^{2}=1 \\
& \int d^{4} y e^{i\left(P+q-P_{X}\right) \cdot y}=(2 \pi)^{4} \delta^{4}\left(P_{X}-P-q\right) .
\end{aligned}
$$

Calculamos entonces

$$
\begin{aligned}
I_{1} \equiv & i \mathcal{Q} \int d^{10} x \sqrt{-g} A_{\mu}\left(\phi_{i} \partial^{\mu} \phi_{X}^{*}-\phi_{X}^{*} \partial^{\mu} \phi_{i}\right) \\
= & (2 \pi)^{4} \delta^{4}\left(P_{X}-P-q\right) \tilde{\beta} n_{\mu} \\
& \times \int_{r_{0}}^{\infty} d r r^{-(\Delta+2)} K_{1}\left(\frac{q R^{2}}{r}\right) J_{\Delta-2}\left(\frac{s^{1 / 2} R^{2}}{r}\right)\left(2 P^{\mu}+q^{\mu}\right),
\end{aligned}
$$

con $\tilde{\beta}=\mathcal{Q} c_{i} c_{X} R^{2} q s^{1 / 4} \Lambda^{-1 / 2} r_{0}^{\Delta}$. Hacemos el cambio de variables $z=R^{2} / r$ y aproximamos $1 / \Delta \rightarrow \infty$. Esta aproximación es válida, porque para $r<r_{i n t}, A_{\mu}$ y $A_{r}$ decaen muy rápidamente y entonces puede extenderse el límite de integración. Con esto, la integral queda

$$
\begin{aligned}
I_{1} & =(2 \pi)^{4} \delta^{4}\left(P_{X}-P-q\right) \tilde{\beta} n_{\mu}\left(2 P^{\mu}+q^{\mu}\right) \int_{0}^{1 / \Delta} d z z^{\Delta} K_{1}(q z) J_{\Delta-2}\left(s^{1 / 2} z\right) \\
& =(2 \pi)^{4} \delta^{4}\left(P_{X}-P-q\right) \mathcal{Q} c_{i} c_{X} \Lambda^{\Delta-1 / 2} \Gamma(\Delta) 2^{\Delta-1} q^{2} n_{\mu} \frac{s^{\Delta / 2-3 / 4}}{\left(s+q^{2}\right)^{\Delta}}\left(2 P^{\mu}+q^{\mu}\right) .
\end{aligned}
$$

La segunda integral es

$$
\begin{aligned}
I_{2} & \equiv i \mathcal{Q} \int d^{10} x \sqrt{-g} A_{r}\left(\phi_{i} \partial^{r} \phi_{X}^{*}-\phi_{X}^{*} \partial^{r} \phi_{i}\right) \\
& =(2 \pi)^{4} \delta^{4}\left(P_{X}-P-q\right) \mathcal{Q} c_{i} c_{X} \Lambda^{\Delta-1 / 2} \Gamma(\Delta) 2^{\Delta-1} q^{\mu} n_{\mu} \frac{s^{\Delta / 2+1 / 4}}{\left(s+q^{2}\right)^{\Delta}} .
\end{aligned}
$$

donde utilizamos la relación de recursión para las funciones de Bessel: $J_{\alpha}^{\prime}(u)=\frac{\alpha}{u} J_{\alpha}(u)-J_{\alpha+1}(u)$.

Utilizando la relación:

$$
\begin{aligned}
n_{\mu}\left\langle\tilde{J}^{\mu}(q)\right\rangle & =(2 \pi)^{4} \delta^{4}\left(P+q-P_{X}\right) n_{\mu}\left\langle P-q, X\left|J^{\mu}(0)\right| P, \mathcal{Q}\right\rangle e^{i q . y} \\
& =i \mathcal{Q} \int d^{10} x \sqrt{-g} A_{m}\left(\Phi_{i} \partial^{m} \Phi_{X}^{*}-\Phi_{X}^{*} \partial^{m} \Phi_{i}\right),
\end{aligned}
$$

y comparando con 6.1.36, hallamos

$$
\left\langle P+q, X\left|J^{\mu}(0)\right| P, \mathcal{Q}\right\rangle=2^{\Delta} \mathcal{Q} c_{i} c_{X} \Gamma(\Delta)\left(P^{\mu}+\frac{q^{\mu}}{2 x}\right) \Lambda^{\Delta-1 / 2} \frac{s^{\Delta / 2-3 / 4}}{\left(s+q^{2}\right)^{\Delta}} q^{2} .
$$

Para hallar $\operatorname{Im} T^{\mu \nu},(6.1 .5)$, se debe elevar al cuadrado el resultado anterior y sumar sobre las excitaciones radiales. Puede estimarse la densidad de estados por un cutoff en $r_{0}$, de manera que la distancia entre los ceros de la función de Bessel (6.1.24) result: ${ }^{15}$

$$
M_{n}=n \pi \Lambda .
$$

${ }^{15}$ Para argumentos $x \gg\left|n^{2}-\frac{1}{4}\right|$ la función de Bessel se comporta como $J_{n}(x) \simeq \sqrt{\frac{2}{\pi x}} \cos \left(x-\frac{\pi}{2} n-\frac{\pi}{4}\right)$. con esto, los ceros de la función de Bessel se encuentran en los $M_{n}=s_{n}^{1 / 2}$ que cumplen $\cos \left(\frac{s^{1 / 2} R^{2}}{r}-\pi \frac{2(\Delta-2)-1}{4}\right)=0$, que son los valores $M_{n}=n \pi \Lambda+\frac{\pi}{4} \Lambda(2 \Delta-3)$. 


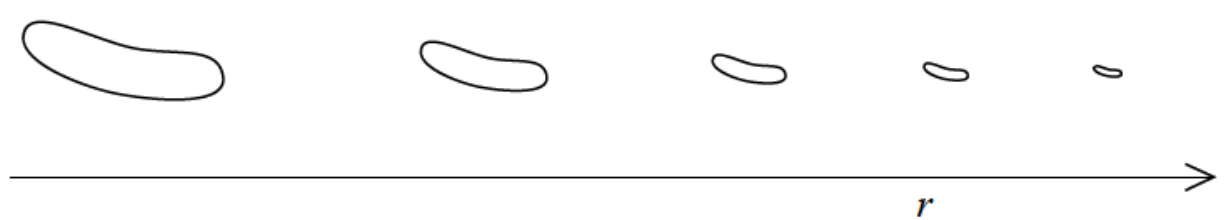

Figura 6.2: Todas las cuerdas dibujadas aquí tienen el mismo tamaño para un observador inercial en diez dimensiones. Vistas desde una posición radial dada $r$, se ven de distintos tamaños. $r \rightarrow \infty$ corresponde al borde del $A d S_{5}$. Figura de [5]

En la aproximación de $N$ grande las funciones de estructura son una suma de funciones delta, pero para $q$ grande se tiene

$$
\sum_{n} \delta\left(M_{n}^{2}-s\right) \sim\left(\frac{\partial M_{n}^{2}}{\partial n}\right)^{-1} \sim\left(2 \pi s^{1 / 2} \Lambda\right)^{-1}
$$

Finalmente, se obtiene como resultado:

$$
\operatorname{Im} T^{\mu \nu}=A_{0} \mathcal{Q}^{2}\left(P^{\mu}+\frac{q^{\mu}}{2 x}\right)\left(P^{\nu}+\frac{q^{\nu}}{2 x}\right) \Lambda^{2 \Delta-2} q^{-2 \Delta} x^{\Delta+2}(1-x)^{\Delta-2} .
$$

El resultado depende de la constante de normalización $A_{0}=2^{2 \Delta} \pi\left|c_{i}\right|^{2}\left|c_{X}\right|^{2} \Gamma(\Delta)^{2}$. Se obtienen como funciones de estructura:

$$
F_{1}=0, \quad F_{2}=A_{0} \mathcal{Q}^{2}\left(\frac{\Lambda^{2}}{q^{2}}\right)^{\Delta-1} x^{\Delta+1}(1-x)^{\Delta-2} .
$$

Este resultado representa la dispersión inelástica profunda de un leptón por un campo escalar, en el régimen $N \gg \lambda \gg 1$. El resultado (6.1.45) corresponde a un proceso de DIS donde el leptón es dispersado por el hadrón de espín cero completo, que cambia de estado, a un estado excitado $\Phi_{X}$, pero no se fragmenta.

La dependencia de estas funciones con $q^{2}$ es la misma que se dedujo usando la expansión del producto de operadores para el límite de $N$ grande y acoplamiento fuerte en la Sección 4.4. Corresponde al segundo término en Eq. 4.4.12), proveniente de los operadores de doble traza con twist $2 \tau_{p}$. Representa la colisión inelástica del electrón por un hadrón entero.

Desde el punto de vista de teoría de cuerdas, un análisis superficial sugeriría una amplitud que decaiga exponencialmente con $q^{2}$, pues la cuerda no tiene partones. Pero dado que la geometría no es plana, sino que posee el factor de deformación $\frac{r^{2}}{R^{2}}$, se genera una dependencia en ley de potencias. Esto es porque la tensión de la cuerda se comporta como $T=\frac{r^{2}}{R^{2}} \tilde{T}$, donde $\tilde{T}$ es la tensión de la cuerda vista desde un sistema inercial en diez dimensiones y $T$ es la misma magnitud medida desde las coordenadas $y^{\mu}$ en cuatro dimensiones. Se observa que la tensión en cuatro dimensiones aumenta como $r^{2}$. Esto influye, a su vez, en que la longitud característica de la cuerda en el espacio de cuatro dimensiones decrezca al aumentar $r$, mientras que es constante para un observador inercial en diez dimensiones, como se ve en la Figura 6.2. La forma más eficiente para que una cuerda realice una dispersión dura es que llegue, mediante el efecto túnel, a un $r$ tal que su tamaño sea del orden de la transferencia de momento.

Para el caso de colisiones elásticas [67], tanto en el límite de partones como en el de cuerdas, la dispersión para intercambios de momento grandes implica que el hadrón entero reduzca su tamaño. En ambos casos, la magnitud de las funciones de onda en esta región queda determinado por la dimensión conforme de los operadores invariantes de gauge que pueden crear el estado. En el caso inelástico, en cambio, no es necesario que el hadrón entero reduzca su tamaño, 
pues el fotón podría interactuar con una fracción del hadrón total, dando lugar a otra escala dimensional. En el límite de partones, el fotón puede impactar un solo partón. La probabilidad de que un partón sea pequeño mientras el resto del hadrón es grande es claramente mayor que la probabilidad de que todo el hadrón sea pequeño: es por esto que domina la colisión monopartónica. En el límite de cuerdas, sin embargo, la probabilidad de que una fracción pequeña de la cuerda tenga un valor grande de $r$ mientras el resto de la cuerda tiene un valor pequeño de $r$ está muy suprimida (ver Figura 6.2). Esto implica que el hadrón en cuatro dimensiones no contiene partones puntuales a grandes valores del parámetro de 't Hooft.

Por otro lado, vemos que la función $F_{1}$, proporcional al operador de Casimir del grupo de Lorentz del objeto dispersado, se anula para este régimen ${ }^{16}$

\section{2. $\quad$ DIS desde mesones escalares}

En 12$]$ se calculó el espectro de masas para distintos tipos de mesones a través de su descripción dual holográifca. En esta Sección obtendremos las funciones de estructura del tensor hadrónico para DIS para estos mesones escalares, y en la próxima Sección para el caso de mesones vectoriales. Desarrollaremos un método sistemático para derivar el lagrangiano de interacción $\mathcal{L}_{\text {int }}$ en la teoría dual holográfica, de una forma complementaria a la propuesta por Polchinski y Strassler [5]. El lagrangiano de interacción obtenido con nuestro método coincide exactamente para el caso de los mesones escalares con el obtenido por Polchinski y Strassler para glueballs, también de espín cero [5]. El enfoque novedoso presentado aquí garantiza la existencia de una corriente conservada en ocho dimensiones ( $p+1$ dimensiones en el caso general de una $\mathrm{D} p$-brana de sabor) directamente derivada de la acción de Dirac-Born-Infeld. A partir de esta corriente es claro el hecho de que se cumpla la condición $q_{\mu} W^{\mu \nu}=0$, necesaria para el tensor hadrónico. Más aún, el lagrangiano de interacción encontrado es invariante ante transformaciones de paridad e inversión temporal. Con esto, el tensor hadrónico satisface todas las propiedades de simetría requeridas. Todas estas pruebas de consistencia confirman la validez del procedimiento por nosotros desarrollado. Los resultados presentados en esta Sección y en la siguiente fueron publicados en [1]. Recordar que en la Sección 5.2.1 se presentaron las características básicas de este modelo holográfico dual.

\section{Cálculo de los campos involucrados}

Las ecuaciones de movimiento para los mesones escalares se obtienen de efectuar fluctuaciones transversales de la D7-brana [12],

$$
Z^{5}=0+2 \pi \alpha^{\prime} \chi, \quad Z^{6}=L+2 \pi \alpha^{\prime} \varphi,
$$

donde las coordenadas $Z^{5}$ y $Z^{6}$ son las que generan el plano $(8,9)$, transversal a la D7-brana. El lagrangiano para las fluctuaciones escalares $\chi$ y $\varphi$ puede derivarse fácilmente de la acción

\footnotetext{
${ }^{16}$ En [5] se realiza también el cálculo de $F_{1}$ y $F_{2}$ para un dilatino (de espín $\frac{1}{2}$ ) no polarizado. Aquí se ve que el resultado para $F_{2}$ coincide en el caso del dilatón y el del dilatino. Esto se debe a que esta función mide información independiente del espín. También se obtiene la relación tipo Callan-Gross $F_{2}=2 F_{1}$ para el caso de espín $\frac{1}{2}$, que obtendremos para mesones vectoriales en la Sección 6.3. La diferencia entre esta relación y la válida en el caso de bariones, $F_{2}=2 x F_{1}$, se debe a que en el régimen cinemático para $x$ y $\lambda$ suficientemente pequeños de los experimentos clásicos de DIS sobre bariones se observa una colisión por un partón, de momento $x P^{\mu}$, en lugar de un hadrón entero, de momento $P^{\mu}$ como en el caso que estudiamos en esta Tesis y en [5] . Este factor $x$ faltante es el que da cuenta en la diferencia entre ambas relaciones de Callan-Gross.
} 
5.2 .5 ,

$$
\mathcal{L}=-\mu_{7} \sqrt{|\operatorname{det}(g)|} \sqrt{\operatorname{det}\left(\delta^{a b}+\left(g^{a c} R^{2} / r^{2}\right)\left(2 \pi \alpha^{\prime}\right)^{2}\left(\partial_{c} \chi \partial^{b} \chi+\partial_{c} \varphi \partial^{b} \varphi\right)\right)} .
$$

Todos los índices pertenecen al volumen de mundo de la D7-brana, consistentemente con la convención definida en la Sección 6.1. Si expandimos este lagrangiano hasta orden cuadrático en las derivadas de las fluctuaciones, este se lee

$$
\mathcal{L}=-\mu_{7} \sqrt{|\operatorname{det}(g)|}\left[1+\frac{2 R^{2}}{r^{2}} g^{a b}\left(\pi \alpha^{\prime}\right)^{2}\left(\partial_{b} \chi \partial_{a} \chi+\partial_{b} \varphi \partial_{a} \varphi\right)\right],
$$

que solo depende de derivadas de los escalares. Sustituyendo $r^{2}=\rho^{2}+L^{2}$ y la métrica inducida (5.2.2) en este lagrangiano cuadrático obtenemos las ecuaciones de movimiento para las fluctuaciones escalares de la D7-brana en la aproximación de prueba, a saber

$$
\partial_{a}\left(\frac{\rho^{3} \sqrt{\operatorname{det} \tilde{g}}}{\rho^{2}+L^{2}} g^{a b} \partial_{b} \Phi\right)=0,
$$

donde $\Phi$ es cualquiera de las fluctuaciones $(\chi \circ \varphi) \sqrt{17}$, mientras que $\tilde{g}_{i j}$ es la métrica de la 3-esfera de radio unidad, cuya coordenada radial $\rho$ se define en el espacio generado por las coordenadas $\left(Z^{1}, \cdots, Z^{4}=x^{4}, \ldots, x^{7}\right)$. Las EOM pueden escribirse más explícitamente como

$$
\frac{R^{4} \rho}{\left(\rho^{2}+L^{2}\right)} \eta^{\mu \nu} \partial_{\mu} \partial_{\nu} \Phi+\partial_{\rho}\left(\frac{\rho^{5}}{\left(\rho^{2}+L^{2}\right)} \partial_{\rho} \Phi\right)+\frac{\rho^{3}}{\left(\rho^{2}+L^{2}\right)} \nabla^{i} \nabla_{i} \Phi=0
$$

donde denotamos como $\nabla_{i}$ a la derivada covariante sobre la esfera $S^{3}$.

Como estamos interesados en la solución para mesones escalares en la región donde

$$
r_{\text {int }}=\sqrt{\rho_{\text {int }}^{2}+L^{2}} \sim q R^{2} \gg r_{0} \equiv \Lambda R^{2}>\sqrt{2} L \Rightarrow \rho_{\text {int }} \gg L,
$$

siendo $r_{\text {int }}$ el valor de la coordenada radial en la cual se produce la interacción y $\Lambda$ un cutoff infrarrojo para la teoría de gauge. En esta región la métrica puede considerarse $A d S_{5} \times S^{3}$, lo que refleja el hecho de que para energías lo suficientemente grandes $\left(E \gg m_{q}\right)$, la invariancia conforme se restituye. La EOM resultante tras esta simplificación es

$$
\frac{R^{4}}{\rho} \eta^{\mu \nu} \partial_{\mu} \partial_{\nu} \Phi+\partial_{\rho}\left(\rho^{3} \partial_{\rho} \Phi\right)+\rho \nabla^{i} \nabla_{i} \Phi=0
$$

Proponemos una solución separable de la forma

$$
\Phi^{\ell}=\phi(\rho) e^{i P \cdot y} Y^{\ell}\left(S^{3}\right),
$$

donde $Y^{\ell}\left(S^{3}\right)$ son los armónicos esféricos de $S^{3}$, con autovalores

$$
\nabla^{i} \nabla_{i} Y^{\ell}\left(S^{3}\right)=-\ell(\ell+2) Y^{\ell}\left(S^{3}\right) .
$$

A partir de aquí simplificaremos la notación, redefiniendo $\Phi \equiv \Phi^{\ell}$. Este índice $\ell$ reaparecerá en el resultado para las funciones de estructura.

\footnotetext{
${ }^{17}$ También podría considerarse $\Phi=\frac{1}{\sqrt{2}}(\chi+i \varphi)$. Esta definición sería útil para este caso pero no para el de $D 4 D 8 \overline{D 8}$ y $D 4 D 6 \overline{D 6}$.
} 
Con esto, el lagrangiano 6.2 .3 para el escalar complejo $\Phi$ en la región de interacción queda ${ }^{18}$

$$
\mathcal{L}=-\mu_{7}\left(\pi \alpha^{\prime}\right)^{2} \sqrt{|\operatorname{det} g|} \frac{R^{2}}{\rho^{2}} g^{a b}\left(\partial_{a} \Phi \partial_{b} \Phi^{*}+\partial_{b} \Phi \partial_{a} \Phi^{*}\right) .
$$

Reemplazando la solución propuesta (6.2.8) en la ecuación (6.2.7), resulta para la parte radial

$$
\partial_{\rho}^{2} \phi+3 \rho^{-1} \partial_{\rho} \phi+\left(-P^{2} R^{4} \rho^{-4}-m_{\ell}^{2} \rho^{-2}\right) \phi=0,
$$

con $m_{\ell}^{2}=\ell(\ell+2)$.

Nuevamente, utilizamos la solución completa para el estado intermedio $X$ con cuadrimomento $P_{X}$ y la solución asintótica con $\rho \sim \rho_{i n t}$ para el hadón inicial y final $\Phi_{i}$, de cuadrimomento $P$. Obtenemos

$$
\begin{gathered}
\Phi_{i}=c_{i}(\Lambda \rho)^{-\gamma-1} e^{i P \cdot y} Y(\Omega) \\
\Phi_{X}=c_{X} \Lambda^{-3 / 2} s^{1 / 4} \rho^{-1} J_{\gamma}\left(\frac{s^{1 / 2} R^{2}}{\rho}\right) e^{i P_{X} \cdot y} Y(\Omega),
\end{gathered}
$$

donde $J_{\gamma}$ es la función de Bessel de primera especie, de orden $\gamma=\sqrt{m_{\ell}^{2}+1}=\ell+1 \mathrm{y}$ $s=-(P+q)^{2}=M_{X}^{2}$ es el cuadrado de la masa del estado intermedio, mientras que $c_{X}$ y $c_{i}$ son constantes adimensionales. Podemos estudiar también la paridad de los mesones considerando que los armónicos esféricos transforman de la siguiente manera: $\left[Y^{\ell}(\Omega)\right]_{\mathrm{P}}=(-1)^{\ell} Y^{\ell}(\Omega)$. Por lo tanto, estos mesones serán escalares y pseudoescalares para valores pares e impares de $\ell$ respectivamente. Continuaremos refiriéndonos a estos como escalares en ambos casos, por simplicidad.

Utilizamos ahora la misma perturbación a la métrica (6.1.11) que en la Sección 6.1, solo que ahora esta solo se produce en las direcciones $0, \ldots, 7$ correspondientes a la métrica inducida sobre el volumen de mundo de la D7-brana de prueba. Obtenemos el lagrangiano de interacción ${ }^{19}$

$$
\mathcal{L}_{i n t}=i \mathcal{Q} \mu_{7}\left(\pi \alpha^{\prime}\right)^{2} \sqrt{|\operatorname{det} g|} R^{2} \rho^{-2} A^{m}\left(\Phi \partial_{m} \Phi_{X}^{*}-\Phi_{X}^{*} \partial_{m} \Phi\right) .
$$

La dependencia angular en los armónicos esféricos corresponde a funciones que son autoestados de carga, con autovalor $\mathcal{Q}$ bajo el grupo de simetría $U(1)$ correspondiente a la dirección del vector de Killing $v_{j}$.

Otra forma de derivar $\mathcal{L}_{\text {int }}$ es a partir del acoplamiento del campo de gauge $A_{m}$ con la corriente de Noether correspondiente a la simetría interna de la acción (6.2.10) bajo transformaciones de fase globales asociadas al grupo $U(1){ }^{20}$, generado por el vector de Killing $v_{j}$. Esta corriente de Noether es

$$
j^{m}=\frac{\partial \mathcal{L}}{\partial\left(\partial_{m} \Phi\right)} \Delta \Phi+\frac{\partial \mathcal{L}}{\partial\left(\partial_{m} \Phi^{*}\right)} \Delta \Phi^{*} .
$$

donde definimos la transformación

$$
\begin{aligned}
& \Phi \rightarrow \Phi+\alpha \Delta \Phi ; \quad \Delta \Phi=-\frac{i}{2} \Phi, \\
& \Phi^{*} \rightarrow \Phi^{*}+\alpha \Delta \Phi^{*} \quad ; \quad \Delta \Phi^{*}=\frac{i}{2} \Phi^{*} .
\end{aligned}
$$

\footnotetext{
${ }^{18}$ En realidad estamos considerando $\Phi=\sum_{\ell} a_{\ell} \Phi^{\ell}$, donde cada uno de los $\Phi^{\ell}$ es claramente complejo debido al armónico esférico que lo compone.

${ }^{19} \mathrm{La}$ unidad imaginaria $i$ se debe a la presencia de una derivada $\partial_{m}$ en cada término de interacción. El lagrangiano resulta real.

${ }^{20} \mathrm{El}$ grupo de isometrías de la esfera $S^{3}$ es $S O(4)$, que es isomorfo a $S U(2) \times S U(2)$, y este contiene un producto $U(1) \times U(1)$. El grupo $U(1)$ que da la presente simetría global es uno de estos, subgrupo del grupo de isometrías [68.
} 
Entonces la corriente de Noether es

$$
j^{m}=i \mu_{7}\left(\pi \alpha^{\prime}\right)^{2} R^{2} \rho^{-2}\left(\Phi \partial^{m} \Phi_{X}^{*}-\Phi_{X}^{*} \partial^{m} \Phi\right) .
$$

Si definimos $\mathcal{L}_{\text {int }}=\mathcal{Q} \sqrt{|\operatorname{det} g|} A^{m} j_{m}$, obtendremos el mismo $\mathcal{L}_{\text {int }}$ de la ecuación (6.2.14) proveniente de la fluctuación de la métrica. Demostramos entonces que el $\mathcal{L}_{\text {int }}$ calculado viene dado por el acoplamiento del campo de gauge $A^{m}$ con la corriente de Noether conservada $j_{m}$. Un punto fundamental en la derivación es que los campos (6.2.8) están cargados ante el grupo $U(1)$ generado por el vector de Killing $v^{j}$ para un $j$ fijo arbitrario. Consideraremos $\ell>0$, lo que nos dice que esta carga es no nula para el modo que estamos estudiando.

Estamos ahora en condiciones de realizar el cálculo holográfico, utilizando la prescripción 6.1 .35

$$
\begin{aligned}
S_{i n t} & =n_{\mu}\left\langle P_{X}, X\left|\tilde{J}^{\mu}(q)\right| P, \mathcal{Q}\right\rangle \\
& =(2 \pi)^{4} \delta^{4}\left(P_{X}-P-q\right) n_{\mu}\left\langle P+q, X\left|J^{\mu}(0)\right| P, \mathcal{Q}\right\rangle .
\end{aligned}
$$

Necesitamos entonces calcular $S_{\text {int }}$, para lo que necesitaremos la contracción $A_{m} j^{m}$. Utilizamos la solución para el campo de gauge calculada en la Sección 6.1, dado que se trata del mismo fondd 21

$$
\begin{aligned}
& A_{\mu}=n_{\mu} e^{i q \cdot y} \frac{q R^{2}}{\rho} K_{1}\left(\frac{q R^{2}}{\rho}\right), \\
& A_{\rho}=-\frac{i}{q^{2}} \eta^{\mu \nu} q_{\mu} \partial_{\rho} A_{\nu}
\end{aligned}
$$

donde $A_{m}=\left(A_{\mu}, A_{\rho}\right)$.

Aplicando la ecuación para la conservación de la corriente $D_{a} J^{a}=0$ resulta $\partial_{\nu} j^{\nu}+$ $\rho^{-3} \partial_{\rho}\left(\rho^{3} j^{\rho}\right)=0$, de donde obtenemos

$$
A_{m} j^{m}=A_{\mu}\left(j^{\mu}-i \frac{q^{\mu}}{q^{2}} \partial_{\gamma} j^{\gamma}\right)-i \frac{q^{\mu}}{q^{2} \rho^{3}} \partial_{\rho}\left(A_{\mu} \rho^{3} j^{\rho}\right),
$$

en términos de $A_{\mu}$ solamente. La acción de interacción queda escrita entonces

$$
\begin{aligned}
S_{\text {int }}= & \int_{r_{0}}^{\infty} d^{8} x \mathcal{Q} \sqrt{|\operatorname{det} g|} A_{m} j^{m} \\
= & \int_{r_{0}}^{\infty} d^{8} x 2 \mu_{7}\left(\pi \alpha^{\prime}\right)^{2} \mathcal{Q} \sqrt{|\operatorname{det} \tilde{g}|} \rho^{-1} R^{4}\left(P^{\mu}+\frac{q^{\mu}}{2 x}\right) A_{\mu} \Phi \Phi_{X}^{*} \\
& -\left.i \frac{q^{\mu}}{q^{2} R^{3}} \int d^{4} y d \Omega \mathcal{Q} \sqrt{|\operatorname{det} \tilde{g}|}\left(A_{\mu} \rho^{3} j^{\rho}\right)\right|_{\Lambda R^{2}} ^{\infty} .
\end{aligned}
$$

Esta integral puede calcularse con la sustitución $z \equiv R^{2} / \rho$ y tomando $1 / \Lambda \rightarrow \infty$ pues $A_{\mu}$ tiende a cero muy rápidamente para $\rho<\rho_{\text {int }}$. Esto último es similar al caso de la Sección 6.1. Vemos que el segundo término de 6.2.21) evaluado en los límites de integración se anula. Integrando el otro término y usando 6.1 .35 resulta

$$
\left\langle P+q, X\left|J^{\mu}(0)\right| P, \mathcal{Q}\right\rangle=\kappa_{1} I(x, \gamma, q)\left(P^{\mu}+\frac{q^{\mu}}{2 x}\right),
$$

\footnotetext{
${ }^{21}$ Obviamente, no es el mismo fondo del espacio total pero, dado que no tenemos en cuenta la backreaction de las $D 7$-branas la geometría, por ser su número $1=N_{f} \ll N$, la parte de la métrica correspondiente al $A d S_{5}$ será igual al caso de la Sección 6.1, y es en esta parte donde está definido el campo de gauge $A_{m}$.
} 
donde $\kappa_{1}=2 \mu_{7} \mathcal{Q}\left(\pi \alpha^{\prime}\right)^{2} c_{i} c_{X}^{*} \Lambda^{\gamma-5 / 2} q^{3 / 2} x^{-1 / 4}(1-x)^{1 / 4}$ y el resultado de la integral en $\rho$ es $I(x, \gamma, q)$. Para $|t| \ll 1$ podemos aproximar $s \simeq q^{2}(1 / x-1)(6.1 .8)$, obteniendo entonces

$$
I(x, \gamma, q)=2^{1+\gamma} \Gamma(2+\gamma) q^{-3-\gamma}(1-x)^{\gamma / 2} x^{\gamma / 2+2} .
$$

Para calcular $\operatorname{Im} T^{\mu \beta}$ como en [5], tenemos que multiplicar 6.2 .22 por su complejo conjugado y sumar sobre las excitaciones radiales. Definimos nuevamente la densidad de estados con el cutoff IR $r_{0}$. La distancia entre los ceros de la función de Bessel en (6.2.13) resulta $M_{n}=n \pi \Lambda$, que en el límite de $N$ grande y para grandes valores de $q$ vuelve a ser, como en [1, 5]

$$
\sum_{n} \delta\left(M_{n}^{2}-s\right) \sim\left(\frac{\partial M_{n}^{2}}{\partial n}\right)^{-1} \sim\left(2 \pi s^{1 / 2} \Lambda\right)^{-1}
$$

Uniendo todos estos resultados tenemos

$$
\begin{aligned}
\operatorname{Im} T^{\mu \beta}= & 4 \pi^{5} \mathcal{Q}^{2} 2^{2+2 \gamma}[\Gamma(2+\gamma)]^{2} \mu_{7}^{2}\left(\alpha^{\prime}\right)^{4}\left|c_{i}\right|^{2}\left|c_{X}\right|^{2} \\
& \times \Lambda^{2 \gamma-6} q^{-4-2 \gamma}(1-x)^{\gamma} x^{\gamma+4}\left(P^{\gamma}+\frac{q^{\mu}}{2 x}\right)\left(P^{\beta}+\frac{q^{\beta}}{2 x}\right) .
\end{aligned}
$$

Solo resta obtener las funciones de estructura para los mesones escalares a partir de la ecuación 4.2.14. Puede verse que $W^{\mu \beta}$ satisface las propiedades de simetría pertinentes y las funciones de estructura resultan

$$
F_{1}=0, \quad F_{2}=A_{0}^{\prime} \mathcal{Q}^{2}\left(\frac{\mu_{7}^{2} \alpha^{\prime 4}}{\Lambda^{8}}\right)\left(\frac{\Lambda^{2}}{q^{2}}\right)^{\gamma+1} x^{\gamma+3}(1-x)^{\gamma},
$$

donde $A_{0}^{\prime}=\pi^{5} 2^{4+2 \gamma}\left|c_{i}\right|^{2}\left|c_{X}\right|^{2}[\Gamma(2+\gamma)]^{2}$ es una constante de normalización adimensional.

\subsection{DIS desde mesones vectoriales}

Nos concentraremos ahora en la derivación de las funciones de estructura para mesones vectoriales polarizados en el modelo con D3D7-branas. En primer lugar derivaremos $\mathcal{L}_{\text {int }}$ para el acoplamiento del campo de gauge $A_{m}$ a los mesones vectoriales. Para esto debemos encontrar primero expresiones explícitas para estos mesones. A partir de estos escribiremos la interacción entre ambos campos y de allí derivaremos las funciones de estructura en el presente caso, partiendo de ideas similares a las desarrolladas en la Sección anterior. Este caso de DIS desde mesones vectoriales polarizados no había sido tratado en la literatura previa a nuestro trabajo [1].

El espectro de mesones vectoriales en este background fue estudiado en [12]. Estos surgen de fluctuaciones de los campos vectoriales de la acción de Dirac-Born-Infeld (DBI) correspondiente a la D7-brana en las direcciones paralelas a esta. Partimos de la acción (5.2.5) y deducimos de esta las EOM. Luego, definimos un lagrangiano de segundo orden que reproduzca las mismas EOM. Partimos entonces de 5.2.5

$$
\mathcal{L}_{D 7}=-\mu_{7} \sqrt{-\operatorname{det}\left(g_{a b}+2 \pi \alpha^{\prime} F_{a b}\right)}+\frac{\left(2 \pi \alpha^{\prime}\right)^{2}}{2} \mu_{7} P\left[C^{(4)}\right] \wedge F \wedge F,
$$

donde $F^{a b} \equiv \partial^{a} B^{b}-\partial^{b} B^{a}$ y los subíndices $D B I$ y $W Z$ denotan la acción de Dirac-Born-Infeld y de Wess-Zumino respectivamente. Utilizando siguientes propiedades

$$
\begin{aligned}
\frac{d}{d \alpha}(\operatorname{det} V) & =(\operatorname{det} V) \operatorname{Tr}\left(V^{-1} \frac{d V}{d \alpha}\right), \\
\left(V_{0}+\epsilon X\right)^{-1} & =V_{0}^{-1}-\epsilon V_{0}^{-1} X V_{0}^{-1}+\mathcal{O}\left(\epsilon^{2}\right),
\end{aligned}
$$


donde $V$ y $X$ son matrices de $n \times n$, y $\epsilon$ es un escalar real infinitesimal, se halla la ecuación de movimiento

$$
\partial_{a}\left(\sqrt{|\operatorname{det} g|} F^{a b}\right)-\frac{4}{R^{4}} \rho\left(\rho^{2}+L^{2}\right) \varepsilon^{b j k} \partial_{j} B_{k}=0,
$$

donde $\varepsilon^{i j k}$ es el pseudotensor de Levi-Civita y la convención sobre los índices es la definida en la Sección 6.1. El segundo término, proveniente de $S_{W Z}$ solo está presente para los valores de $b$ definidos sobre la $S^{3}$. La solución que corresponde a los mesones vectoriales $B_{\mu}$ se propone utilizando separación de variables,

$$
B_{\mu}=\zeta_{\mu} \phi(\rho) e^{i P \cdot y} Y^{\ell}\left(S^{3}\right), \quad P \cdot \zeta=0, \quad B_{\rho}=0, \quad B_{i}=0,
$$

donde nuevamente hicimos una expansión en armónicos esféricos de $S^{3}, \phi(\rho)$ es la dependencia radial por determinar y $\zeta_{\mu}$ es el vector polarización, que cumple la condición de transversalidad $\zeta \cdot P=0$ proveniente de $\partial^{\mu} B_{\mu}=0$. Reemplazando la solución propuesta en 6.3 .3 , se tiene:

$$
\begin{aligned}
\frac{1}{\sqrt{|\operatorname{det} \tilde{g}|}} \partial_{\mu}\left[\sqrt{|\operatorname{det} g|}\left(\partial^{\mu} B^{b}-\partial^{b} B^{\mu}\right)\right]+\frac{1}{\sqrt{|\operatorname{det} \tilde{g}|}} \partial_{\rho}\left[\sqrt{|\operatorname{det} g|} \partial^{\rho} B^{b}\right] & \\
+\frac{\rho\left(\rho^{2}+L^{2}\right)}{R^{2}} \nabla_{i} \nabla^{i} B^{b} & =0,
\end{aligned}
$$

donde $\tilde{g}$ es la métrica sobre $S^{3}$ y $g$ la métrica inducida sobre la D7 brana. Los armónicos esféricos cumplen $\nabla_{S^{d-1}}^{2} Y^{\ell}\left(S^{d-1}\right)=-\ell(\ell+d-2) Y^{\ell}\left(S^{d-1}\right)$, que para $d=4$ es

$$
\nabla_{S^{3}}^{2} Y^{\ell}\left(S^{3}\right)=-\ell(\ell+2) Y^{\ell}\left(S^{3}\right),
$$

con lo cual $\nabla_{i} \nabla^{i} B^{\mu}=-\ell(\ell+2) B^{\mu}$. Las soluciones con $b=k$ y $b=\rho$ se satisfacen idénticamente a partir de la condición $\partial^{\mu} B_{\mu}=0$. Para $b=\mu$ se tiene

$$
\frac{R^{2}}{\rho\left(\rho^{2}+L^{2}\right)} \frac{1}{\sqrt{|\operatorname{det} \tilde{g}|}} \partial_{m}\left(\sqrt{|\operatorname{det} g|} \partial^{m} B^{\mu}\right)-m_{\ell}^{2} B^{\mu}=0,
$$

donde $m_{\ell}^{2}=\ell(\ell+2)$.

La región de interacción corresponde nuevamente a $\rho_{\text {int }} \gg L>0$, donde la energía de cuatro dimensiones es $E \gg \Lambda$. De manera análoga al caso de mesones escalares, puede aproximarse la métrica por $A d S_{5} \times S^{3}$. En esta región de interacción la EOM resultante para $b=\mu$ es

$$
\frac{1}{\sqrt{\left|\operatorname{det} g_{A d S}\right|}} \partial_{m}\left(\sqrt{\left|\operatorname{det} g_{A d S}\right|} \partial^{m} B^{\mu}\right)-\frac{m_{\ell}^{2}}{R^{2}} B^{\mu}=0,
$$

donde $g_{A d S}$ es la métrica del $A d S_{5}$ y la convención de índices es la usual. Reemplazando la propuesta 6.3.4 en (6.3.8) recuperamos la ecuación (6.2.11) para la dependencia radial, cuya solución es

$$
\phi(\rho)=\frac{c}{\rho} J_{\gamma}\left(\frac{s^{1 / 2} R^{2}}{\rho}\right),
$$

donde $J_{\gamma}$ es la función de Bessel de primera especie, $c$ una constante adimensional y $\gamma=\ell+1$. Nuevamente, para los estados hadrónicos inicial y final consideramos solamente el comportamiento dominante con $|t|=\frac{\left|P^{2}\right|}{q^{2}} \ll 1$, la solución es

$$
B_{\mu I N / O U T}=\zeta_{\mu} c_{i} \Lambda^{-1}(\Lambda \rho)^{-\gamma-1} e^{i P \cdot y} Y^{\ell}\left(S^{3}\right),
$$


donde $c_{i}$ es una constante adimensional. Para el estado intermedio consideramos la solución completa, por las mismas consideraciones que las expuestas en la Sección anterior,

$$
B_{X \mu}=\zeta_{X \mu} c_{X} \frac{s^{-1 / 4} \Lambda^{-3 / 2}}{\rho} J_{\gamma}\left(\frac{s^{1 / 2} R^{2}}{\rho}\right) e^{i P_{X} \cdot y} Y^{\ell}\left(S^{3}\right)
$$

donde $c_{X}$ es una constante adimensional. Podemos estudiar el comportamiento de estos mesones ante transformaciones de paridad utilizando $\left[Y^{\ell}(\Omega)\right]_{\mathrm{P}}=(-1)^{\ell} Y^{\ell}(\Omega)$ y $\left[\zeta_{\mu}\right]_{\mathrm{P}}=\left(\zeta^{0},-\vec{\zeta}\right)$. Vemos que tenemos mesones vectoriales para valores pares de $\ell$ y mesones pseudovectoriales (axiales) para valores impares de $\ell$. Utilizaremos el término "mesón vectorial" para referirnos a ambos casos indistintamente, a fin de simplificar la nomenclatura.

De la expansión de $B_{\mu}$ en armónicos esféricos de $S^{3}$, podemos ver que los campos de gauge de la D7-brana corresponden a campos cargados en el $A d S_{5}$. Esto es fácil de ver si consideramos la expansión completa de Kaluza-Klein

$$
B_{\mu}=\sum_{\ell} a_{\ell} B_{\mu}^{\ell}
$$

y luego consideramos transformaciones de la forma $\left(x^{m}, x^{i}\right) \rightarrow\left(x^{m}, x^{i}+v^{i} \varepsilon\left(x^{m}\right)\right)$, donde $v^{i}$ es un vector de Killing de $S^{3}$. Ante esta transformación, encontramos que nuestro campo de gauge definido sobre el espacio $A d S_{5} \times S^{3}$ transforma como

$$
\delta B_{\mu}=-\epsilon\left(x^{m}\right) v^{i} \partial_{i} B_{\mu}-\partial_{\mu} \epsilon\left(x^{m}\right),
$$

y usando la ecuación de autovalores $v^{j} \partial_{j} Y^{\ell}\left(S^{3}\right)=i \mathcal{Q}_{\ell} Y^{\ell}\left(S^{3}\right)$,

$$
\begin{aligned}
\delta B_{\mu}^{0} & =-\partial_{\mu} \epsilon\left(x^{m}\right) & & \ell=0, \\
\delta B_{\mu}^{\ell} & =-\epsilon\left(x^{m}\right) i \mathcal{Q}_{\ell} B_{\mu} & & \ell>0 .
\end{aligned}
$$

Es decir, encontramos un campo de gauge $B_{\mu}^{0}$ en el $A d S_{5}$, dado que transforma como tal ante las tranformaciones $U(1)$ parametrizadas por $\epsilon(x)$. Por otro lado, todos los demás campos $B_{\mu}^{\ell}$, con $\ell>0$ tienen una carga $\mathcal{Q}_{\ell}>0$, pues transforman con una fase ante estas mismas transformaciones. Además, todos estos son campos vectoriales masivos desde el punto de vista del $A d S_{5}$, siendo sus masas adimensionales $m_{\ell}^{2}=\ell(\ell+2){ }_{2}^{22} \mathrm{Su}$ EOM es $6.3 .8{ }^{23}$. Las EOM obtenidas para estos mesones vectoriales en la región de interacción también pueden derivarse del lagrangiano cuadrático

$$
\mathcal{L}=-2 \mu_{7}\left(\pi \alpha^{\prime}\right)^{2} \sqrt{|\operatorname{det} g|} F^{a b} F_{a b}^{*}
$$

Veamos ahora la interacción en el interior del $A d S$. Considerando la fluctuación de la métrica 6.1.11) $\delta g_{m j}=A_{m}(y, r) v_{j}(\Omega)$ y la ecuación de autovalores $v^{j} \partial_{j} Y(\Omega)=i \mathcal{Q} Y(\Omega)$ obtenemos el lagrangiano de interacción

$$
\mathcal{L}_{i n t}=2 i \mathcal{Q} \mu_{7}\left(\pi \alpha^{\prime}\right)^{2} \sqrt{|\operatorname{det} g|} A_{m}\left[B_{X n}^{*} F^{n m}-B_{n}\left(F_{X}^{n m}\right)^{*}\right] .
$$

Este también podría ser obtenido a partir del acoplamiento del campo de gauge $A_{m}$ con la corriente de Noether conservada $j^{m}$ correspondiente a la simetría interna de 6.3.16). Esto corresponde a transformaciones del grupo $U(1)$ y por ende $\mathcal{L}_{i n t}=\mathcal{Q} \sqrt{|\operatorname{det} g|} A_{m} j^{m}$ donde

$$
j^{m}=2 i \mu_{7}\left(\pi \alpha^{\prime}\right)^{2}\left[B_{X n}^{*} F^{n m}-B_{n}\left(F_{X}^{n m}\right)^{*}\right] .
$$

\footnotetext{
${ }^{22}$ No explicitamos el factor dimensional $R^{-2}$. Las masas son estrictamente $\tilde{m}_{\ell}^{2}=\frac{m_{\ell}^{2}}{R^{2}}=\frac{\ell(\ell+2)}{R^{2}}$.

${ }^{23}$ Notaremos en adelante $B_{\mu}^{\ell} \equiv B_{\mu}, \ell>0$, por simplicidad.
} 
Necesitamos ahora calcular este acoplamiento $A_{m} j^{m}$. Utilizando un método similar al de la Sección anterior para mesones escalares, calculamos

$$
\begin{aligned}
S_{\text {int }} & =\int_{r_{0}}^{\infty} d^{8} x \mathcal{Q} \sqrt{|\operatorname{det} g|} A_{m} j^{m} \\
& =\int_{r_{0}}^{\infty} d^{8} x 2 \mu_{7}\left(\pi \alpha^{\prime}\right)^{2} \mathcal{Q} \sqrt{|\operatorname{det} g|} R^{4} \rho^{-4} A_{\mu} N^{\mu} \tilde{B} \tilde{B}_{X}^{*}-\left.i \frac{q^{\mu}}{q^{2} R^{3}} \int d^{4} y d \Omega\left(A_{\mu} \rho^{3} j^{\rho}\right)\right|_{\Lambda R^{2}} ^{\infty},
\end{aligned}
$$

donde definimos $B_{\mu}=\zeta_{\mu} \tilde{B}$ y

$$
N^{\mu}=2\left(\zeta \cdot \zeta_{X}\right)\left(P^{\mu}+\frac{q^{\mu}}{2 x}\right)+\left(\zeta_{X} \cdot q\right) \zeta^{\mu}-(\zeta \cdot q) \zeta_{X}^{\mu}
$$

Nuevamente tomamos $z \equiv R^{2} / \rho$ y $1 / \Lambda \rightarrow \infty$ para poder calcular la integral analíticamente. Nuevamente obtenemos que el segundo término de (6.3.19) se anula. Comparando esto con el Ansatz (6.1.35),

$$
\left\langle P+q, X\left|J^{\mu}(0)\right| P, \mathcal{Q}\right\rangle=\kappa_{2} I(x, \gamma, q) N^{\mu},
$$

donde $\kappa_{2}=2 \mu_{7} \mathcal{Q}\left(\pi \alpha^{\prime}\right)^{2} c_{i} c_{X}^{*} q^{1 / 2} \Lambda^{\gamma-7 / 2} x^{1 / 4}(1-x)^{-1 / 4}$, usando 6.1 .9 resulta

$$
I(x, \gamma, q)=2^{\gamma+1} \Gamma(\gamma+2) q^{-\gamma-3} x^{\gamma / 2+2}(1-x)^{\gamma / 2} .
$$

Para obtener $\operatorname{Im} T^{\mu \nu}$ a partir de (6.1.5) tenemos que multiplicar 6.3 .21$)$ por su complejo conjugado y sumar sobre las excitaciones radiales y las polarizaciones de los estados hadrónicos finales $\zeta_{X}^{\mu}$. La densidad de estados se estima de igual forma que en la Sección anterior para mesones escalares. El resultado para este tensor es

$$
\begin{aligned}
\operatorname{Im} T^{\mu \nu}= & \frac{\pi \kappa_{2} \kappa_{2}^{*} I(x, \gamma, q)^{2}}{\Lambda s^{1 / 2}} \sum_{\lambda} N^{\mu} N^{* \nu} \\
= & \frac{\pi \kappa_{2} \kappa_{2}^{*} I(x, \gamma, q)^{2}}{\Lambda s^{1 / 2}} \sum_{\lambda}\left\{\left[2\left(\zeta \cdot \zeta_{X}\right)\left(P^{\mu}+\frac{q^{\mu}}{2 x}\right)+\left(\zeta_{X} \cdot q\right) \zeta^{\mu}-(\zeta \cdot q) \zeta_{X}^{\mu}\right]\right. \\
& \left.\times\left[2\left(\zeta^{*} \cdot \zeta_{X}^{*}\right)\left(P^{\nu}+\frac{q^{\nu}}{2 x}\right)+\left(\zeta_{X}^{*} \cdot q\right) \zeta^{* \nu}-\left(\zeta^{*} \cdot q\right) \zeta_{X}^{* \nu}\right]\right\}
\end{aligned}
$$

Para la solución (6.3.4) de mesones vectoriales tenemos $P \cdot \zeta=0$, por lo que hay tres polarizaciones permitidas: $\lambda=1,2,3$. Con la normalización $\zeta^{\mu}\left(P_{X}, \lambda\right) \cdot \zeta_{\mu}^{*}\left(P_{X}, \lambda^{\prime}\right)=-M_{X}^{2} \delta_{\lambda, \lambda^{\prime}}$ para las polarizaciones tenemos 69]

$$
\begin{aligned}
\sum_{\lambda} \zeta_{X \mu}\left(P_{X}, \lambda\right) \zeta_{X \nu}^{*}\left(P_{X}, \lambda\right) & =M_{X}^{2} \eta_{\mu \nu}+P_{X \mu} P_{X \nu} \\
& =M_{X}^{2} \eta_{\mu \nu}+\left(P_{\mu}+q_{\mu}\right)\left(P_{\nu}+q_{\nu}\right)
\end{aligned}
$$

Hay que sumar ahora sobre las polarizaciones del estado hadrónico final $X$. Escribimos el tensor $\operatorname{Im} T_{\mu \nu}$ como

$$
\sum_{\lambda} \operatorname{Im} T_{\mu \nu} \equiv \frac{2 \pi \kappa_{2} \kappa_{2}^{*} I(x, \gamma, q)^{2}}{\Lambda s^{1 / 2}} H_{\mu \nu}=\frac{2 \pi \kappa_{2} \kappa_{2}^{*} I(x, \gamma, q)^{2}}{\Lambda s^{1 / 2}}\left(H_{\mu \nu}^{S}+H_{\mu \nu}^{A}\right)
$$


donde lo hemos separado en su parte simétrica y antisimétrica, despreciando los términos proporcionales a $q_{\mu}$ y $q_{\nu}{ }^{24}$. Obtenemos

$$
\begin{aligned}
H_{\mu \nu}^{S}= & -\eta_{\mu \nu}(\zeta \cdot q)\left(\zeta^{*} \cdot q\right)(P+q)^{2}+P_{\mu} P_{\nu}\left[-4 P^{2}(P+q)^{2}+(q . \zeta)\left(q \cdot \zeta^{*}\right)\right] \\
& +\left(\zeta_{\mu} \zeta_{\nu}^{*}+\zeta_{\nu} \zeta_{\mu}^{*}\right) \frac{1}{2}\left[(P \cdot q)^{2}-P^{2} q^{2}\right] \\
& +\left(P_{\mu} \zeta_{\nu}^{*}+P_{\nu} \zeta_{\mu}^{*}\right)(\zeta \cdot q) \frac{1}{2}\left[P \cdot q+q^{2}\right]+\left(P_{\mu} \zeta_{\nu}+P_{\nu} \zeta_{\mu}\right)\left(\zeta^{*} \cdot q\right) \frac{1}{2}\left[P \cdot q+q^{2}\right](6 .
\end{aligned}
$$

y

$$
\begin{aligned}
H_{\mu \nu}^{A}= & \frac{1}{2}\left(\zeta_{\mu} \zeta_{\nu}^{*}-\zeta_{\nu} \zeta_{\mu}^{*}\right)\left[(P \cdot q)^{2}-P^{2} q^{2}\right] \\
& +\left(P_{\nu} \zeta_{\mu}^{*}-P_{\mu} \zeta_{\nu}^{*}\right)(\zeta . q) \frac{1}{2}\left[4 P^{2}+7 P . q+3 q^{2}\right] \\
& +\left(P_{\mu} \zeta_{\nu}-P_{\nu} \zeta_{\mu}\right)\left(\zeta^{*} \cdot q\right) \frac{1}{2}\left[4 P^{2}+7 P . q+3 q^{2}\right] .
\end{aligned}
$$

Debemos reescribir ahora el tensor hadrónico $W_{\mu \nu}$ en 4.3.5 para compararlo con $H_{\mu \nu}$.

Utilizaremos la definición

$$
s^{\sigma}=-\frac{i}{M^{2}} \epsilon^{\sigma \alpha \beta \rho} \zeta_{\alpha}^{*} \zeta_{\beta} P_{\rho}
$$

y las identidades [70]:

$$
\begin{aligned}
-\epsilon_{\mu \nu \lambda \rho} \epsilon^{\alpha \beta \gamma \rho} & =\delta_{\mu \nu \lambda}^{\alpha \beta \gamma}, \\
-\frac{1}{2} \epsilon_{\mu \nu \lambda \rho} \epsilon^{\alpha \beta \lambda \rho} & =\frac{1}{2} \delta_{\mu \nu \lambda}^{\alpha \beta \lambda}=\delta_{\mu \nu}^{\alpha \beta},
\end{aligned}
$$

donde

$$
\begin{aligned}
\delta_{\mu \nu \lambda}^{\alpha \beta \gamma} & = \begin{cases}1 & \text { si } \alpha \beta \gamma \text { es una permutación par de } \mu \nu \lambda, \\
-1 & \text { si } \alpha \beta \gamma \text { es una permutación impar de } \mu \nu \lambda, \\
0 & \text { en otro caso . }\end{cases} \\
\delta_{\mu \nu}^{\alpha \beta} & = \begin{cases}1 & \text { si } \alpha \beta \text { es una permutación par de } \mu \nu, \\
-1 & \text { si } \alpha \beta \text { es una permutación impar de } \mu \nu, \\
0 & \text { en otro caso } .\end{cases}
\end{aligned}
$$

Obtenemos entonces la descomposición

$$
W_{\mu \nu}(P, q)_{h^{\prime} h}^{\text {vector }}=W_{\mu \nu}^{S}(P, q)_{h^{\prime} h}+W_{\mu \nu}^{A}(P, q)_{h^{\prime} h} .
$$

\footnotetext{
${ }^{24}$ Pues sabemos, del Capítulo 4, que estos no contribuirán a la sección eficaz de dispersión
} 
En el límite $|t| \ll 1(\tilde{\kappa} \simeq 1)$ cada contribución se escribe como

$$
\begin{aligned}
W_{\mu \nu}^{S}= & \left\{F_{1}+\left[\frac{\left(q \cdot \zeta^{*}\right)(q \cdot \zeta)}{(P \cdot q)^{2}}-\frac{1}{3}\right] b_{1}+\frac{P^{2}}{9(P \cdot q)}\left(-b_{2}+3 b_{3}\right)\right\} \eta_{\mu \nu} \\
& -\left\{3 F_{2}+\frac{(q \cdot \zeta)\left(q \cdot \zeta^{*}\right)}{(P \cdot q)^{2}}\left(b_{2}+3 b_{3}+3 b_{4}\right)-b_{2}\right\} \frac{P_{\mu} P_{\nu}}{3(P \cdot q)} \\
& +\left(-b_{2}+3 b_{3}\right) \frac{\left(\zeta_{\mu} \zeta_{\nu}^{*}+\zeta_{\mu}^{*} \zeta_{\nu}\right)}{6(P \cdot q)}+\frac{\left(-b_{2}+3 b_{4}\right)}{12(P \cdot q)^{2}}\left[\left(q \cdot \zeta^{*}\right)\left(P_{\mu} \zeta_{\nu}+\zeta_{\mu} P_{\nu}\right)+c . c .\right], \\
W_{\mu \nu}^{A}= & {\left[g_{1}+\frac{q^{2} P^{2}}{(P \cdot q)^{2}} g_{2}\right] \frac{\zeta^{* \mu} \zeta^{\nu}-\zeta^{* \nu} \zeta^{\mu}}{P^{2}}+\left[\left(q \cdot \zeta^{*}\right)\left(\zeta^{\mu} P^{\nu}-\zeta^{\nu} P^{\mu}\right)+c . c .\right] \frac{g_{1}}{P^{2}(P \cdot q)}, }
\end{aligned}
$$

donde c.c. denota el complejo conjugado. Utilizando (4.2.14 y comparando 6.3.26) y 6.3.27) con 6.3.31 y 6.3.31 respectivamente, obtenemos un sistema de seis ecuaciones con ocho incógnitas (las funciones de estructura). Sin embargo, dado que estas funciones no dependen de las polarizaciones, tendremos dos ecuaciones adicionales y por lo tanto las funciones de estructura quedarán unívocamente determinadas. Estas son funciones adimensionales de las variables adimensionales $t=\frac{P^{2}}{q^{2}}<0$ y $x=-\frac{q^{2}}{2 P \cdot q}$, como se definieron en el Capítulo 4 , así como en términos de los parámetros del modelo $\Lambda,\left|c_{i}\right|,\left|c_{X}\right|$ y $\mathcal{Q}$. Los resultados obtenidos para las funciones de estructura son 25

$$
\begin{aligned}
F_{1} & =A(x) \frac{1}{12 x^{3}}\left(1-x-4 x^{2} t+4 x^{3} t\right) \\
F_{2} & =A(x) \frac{1}{6 x^{3}}\left(1-x+12 x t-14 x^{2} t-12 x^{2} t^{2}\right) \\
b_{1} & =A(x) \frac{1}{4 x^{3}}(1-x-x t) \\
b_{2} & =A(x) \frac{1}{2 x^{3}}\left(1-x-x^{2} t\right) \\
b_{3} & =A(x) \frac{1}{24 x^{3}}\left(1-4 x+8 x^{2} t\right) \\
b_{4} & =A(x) \frac{1}{12 x^{3}}\left(-1+4 x-2 x^{2} t\right) \\
g_{1} & =A(x) \frac{t}{8 x^{2}}(-7+6 x+8 x t) \\
g_{2} & =A(x) \frac{1}{16 x^{4}}\left(3-3 x-4 x t+2 x^{2} t\right) .
\end{aligned}
$$

con

$$
A(x)=A_{0} \mathcal{Q}^{2}\left(\frac{\mu_{7}^{2}\left(\alpha^{\prime}\right)^{4}}{\Lambda^{8}}\right)\left(\frac{\Lambda^{2}}{q^{2}}\right)^{\gamma} x^{\gamma+5}(1-x)^{\gamma-1}
$$

y la constante adimensional

$$
A_{0}=\left|c_{i}\right|^{2}\left|c_{X}\right|^{2} 2^{4+2 \gamma}[\Gamma(2+\gamma)]^{2} \pi^{5} .
$$

A partir de estos resultados podemos extraer varias relaciones entre las funciones de estructura del tipo Callan-Gross, válidas en el límite $t \rightarrow 0$. Estas se discutirán en detalle en la Sección 6.7. Es importante notar que en estos resultados la constante $A_{0}$ depende de $\gamma$, que a su vez es función del índice $\ell$, por lo que será distinta para cada mesón de la torre. También $\mathcal{Q}=\mathcal{Q}_{\ell}$ depende de este parámetro.

\footnotetext{
${ }^{25}$ En nuestro trabajo [1] hay un error en $g_{1}$, corregido ya en nuestro trabajo [2], y un error en la parte no dominante de $F_{1}$, que corregimos en [4].
} 


\begin{tabular}{|c|c|c|c|}
\hline Modelo / Parámetro & $p$ & $\alpha$ & $\beta$ \\
\hline$D 3 D 7$ & 7 & 2 & -2 \\
\hline$D 4 D 8 \overline{D 8}$ & 8 & $3 / 2$ & $-3 / 2$ \\
\hline$D 4 D 6 \overline{D 6}$ & 6 & $3 / 2$ & $-3 / 2$ \\
\hline
\end{tabular}

Tabla 6.1: Valores de los parámetros para los distintos modelos.

\subsection{Extensión a modelos más generales}

En este Capítulo estudiaremos un enfoque general para obtener las funciones de estructura para mesones escalares y vectoriales con una sola $\mathrm{D} p$-brana de sabor $N_{f}=1 \mathrm{y}$ para mesones vectoriales con más de una $\mathrm{D} p$-brana de sabor $N_{f}>1$. Estudiaremos en las siguientes Secciones el régimen $x \lesssim 1$ (región $\mathbf{A}$ ), que corresponde a resultados publicados en nuestro trabajo [2]. En el próximo Capítulo se estudiará el caso general para las regiones $\mathbf{B}$ y $\mathbf{C}(x \ll 1)$, correspondientes a resultados de nuestro trabajo [3] (Sección 7.4 de esta Tesis).

Veremos que en el caso general aquí estudiado, que incluye el modelo de D3D7-branas, así como también los modelos de $\mathrm{D} 4 \mathrm{D} 6 \overline{\mathrm{D} 6}$ y $\mathrm{D} 4 \mathrm{D} 8 \overline{\mathrm{D} 8}$, siempre encontraremos las funciones de estructura factorizadas en una parte dependiente del modelo y otra parte independiente de este. En particular, esto permitirá escribir relaciones tipo Callan-Gross similares a las halladas en las Secciones anteriores independientemente del modelo holográfico considerado. También podremos calcular, así como en las Secciones anteriores, las funciones de estructura explícitamente para todos estos modelos.

Partimos de una métrica general de background en diez dimensiones de la forma

$$
d s^{2}=\left(\frac{\rho}{R}\right)^{\alpha} \eta_{\mu \nu} d y^{\mu} d y^{\nu}+\left(\frac{\rho}{R}\right)^{\beta} d \vec{Z} \cdot d \vec{Z}
$$

donde $y^{\mu}=\left(y^{0}, \ldots, y^{3}\right), \vec{Z}=\left(Z^{1}, \ldots, Z^{6}\right)$ y suponemos las condiciones $\alpha>1$ y $\beta<-126$ Agregamos ahora una $\mathrm{D} p$-brana de prueba que da lugar a una métrica inducida de la forma

$$
d s_{D p}^{2}=\left(\frac{\rho}{R}\right)^{\alpha} \eta_{\mu \nu} d y^{\mu} d y^{\nu}+\left(\frac{\rho}{R}\right)^{\beta}\left[d \rho^{2}+\rho^{2} d \Omega_{p-4}^{2}\right]
$$

donde $\rho$ es la dirección radial del volumen de mundo de la $\mathrm{D} p$-brana. El radio $R$ es la escala de longitud del sistema mientras que $\Omega_{p-4}$ indica las coordenadas en la esfera $S^{p-4}$.

Esta métrica general incluye las formas asintóticas de los modelos de D3D7-, D4D8 $\overline{\mathrm{D} 8}$ - y D4D6 $\overline{\mathrm{D} 6}$-branas. Los valores de los parámetros $\alpha, \beta$ у $p$ para cada modelo se ven en la Tabla 6.1. En particular, para el modelo D3D7 la geometría es asintóticamente $A d S_{5} \times S^{3}$ y $R$ es el radio de la $S^{3}$ y del $A d S_{5}$. Para el caso del modelo con D4D8 $\overline{\mathrm{D} 8}$ y de $\mathrm{D} 4 \mathrm{D} 6 \overline{\mathrm{D} 6}$-branas tenemos una métrica que no es asintóticamente conforme. En los tres casos estamos considerando este modelo general como la métrica asintótica, i.e. la correspondiente para $\rho \gg \rho_{0}$ en el caso de D3D7 y $U \gg U_{0}$ en la notación de $\mathrm{D} 4 \mathrm{D} 6 \overline{\mathrm{D} 6}$ y $\mathrm{D} 4 \mathrm{D} 8 \overline{\mathrm{D} 8}$. Utilizaremos $\rho$ para la variable radial en todos los casos, homogeneizando la notación.

Tanto los mesones escalares como los vectoriales corresponden a excitaciones de cuerdas abiertas que terminan en las $\mathrm{D} p$-branas de prueba. La dinámica de estas fluctuaciones está des-

\footnotetext{
${ }^{26}$ Restringiéndonos a los modelos D3D7, D4D8 $\overline{\mathrm{D} 8}$ y D4D6 $\overline{\mathrm{D} 6}$, se cumple siempre que $\alpha=-\beta$. Mantenemos ambas variables como independientes para expresar nuestros resultados en términos más generales.
} 
cripta por la acción

$$
S_{D p}=-\mu_{p} \int d^{p+1} \xi \sqrt{-\operatorname{det}\left(\hat{P}[g]_{a b}+2 \pi \alpha^{\prime} F_{a b}\right)}+\frac{\left(2 \pi \alpha^{\prime}\right)^{2}}{2} \mu_{p} \int \hat{P}\left[C^{(p-3)}\right] \wedge F \wedge F,
$$

donde $g_{a b}$ es la métrica 6.4 .2$), \mu_{p}=\left[(2 \pi)^{p} g_{s} \alpha^{\prime \frac{p+1}{2}}\right]^{-1}$ es la tensión de la $\mathrm{D} p$-brana de prueba y $\hat{P}$ es el pull-back de los campos del background sobre el volumen de mundo de la $\mathrm{D} p$-brana.

Los métodos desarrollados en esta Tesis corresponden a nuestros trabajos [1], 2, , 3] y [4] y pueden extenderse a modelos tales como las extensiones del modelo de Klebanov-Strassler [57] con $\mathrm{D} p$-branas de sabor [59, 60, 61], a las extensiones del modelo de Maldacena-Núñez [58] con Dp-branas de sabor [62] y a otros modelos. En dichos casos los cálculos serán más complicados debido a las geometrías involucradas y habrá dependencia en los parámetros de cada modelo. Sin embargo, se espera que las nuevas relaciones de Callan-Gross generalizadas obtenidas en [1], [2] y [3] y que se describen en esta Tesis persistan. Esto se debe a que en todos los modelos mencionados las branas de sabor se introducen en la aproximación de prueba mediante la acción de Dirac-Born-Infeld correspondiente en cada caso.

\subsubsection{DIS desde mesones escalares para modelos más generales}

Las EOM para mesones escalares se obtienen a partir de fluctuaciones en la $\mathrm{D} p$-brana ortogonales a las direcciones del volumen de mundo de la misma. Tomemos una coordenada $Z^{i}$ en (6.4.1) con algún $i=p+1, \ldots, 9$, de manera que sea perpendicular al volumen de mundo de la $\mathrm{D} p$-brana, y realicémosle una pequeña perturbación de la forma

$$
Z^{i}=Z_{0}^{i}+2 \pi \alpha^{\prime} \Phi
$$

donde $\Phi$ es una fluctuación escalar cuyo lagrangiano se deriva fácilmente de (6.4.3). Basta poner $F_{a b}=0$ y expandir la fluctuación a segundo orden para obtener

$$
S_{0}^{\text {scalar }}=-\mu_{p} \int d^{p+1} \xi \sqrt{-\operatorname{det} g}\left[1+\frac{\left(2 \pi \alpha^{\prime}\right)^{2}}{2}\left(\frac{\rho}{R}\right)^{\beta} g^{a b} \partial_{a} \Phi \partial_{b} \Phi\right],
$$

que corresponde a un lagrangiano para la perturbación

$$
\mathcal{L}_{0}^{\text {scalar }}=-\mu_{p} \sqrt{-\operatorname{det} g}\left[1+\frac{\left(2 \pi \alpha^{\prime}\right)^{2}}{2}\left(\frac{\rho}{R}\right)^{\beta} g^{a b} \partial_{a} \Phi \partial_{b} \Phi\right],
$$

donde todos los índices se refieren a direcciones sobre el volumen de mundo de la $\mathrm{D} p$-brana, notación consistente con la de la Sección 6.1. Esta brana de prueba contiene el espacio de Minkowski tetradimensional y se enrolla sobre la $S^{p-4}$. Insertando la métrica 6.4 .2 en el lagrangiano cuadrático, obtenemos las EOM para las fluctuaciones escalares en la D $p$-brana en la aproximación de prueba

$$
\partial_{a}\left[\left(\frac{\rho}{R}\right)^{\theta-\beta} \sqrt{\widetilde{g}} g^{a b} \partial_{b} \Phi\right]=0
$$

donde hemos definido

$$
\theta=2 \alpha+\left(\frac{p}{2}-\frac{3}{2}\right) \beta+(p-4) .
$$

Notemos que $\widetilde{g}_{i j}$ es la métrica sobre $S^{p-4}$, que junto con $\rho$ barre las coordenadas $\left(Z^{1}, \cdots, Z^{p-3}\right)$. Esta EOM puede ser escrita explícitamente como

$$
\square \Phi+\left(\frac{\rho}{R}\right)^{\alpha-\beta-2} R^{-2} \nabla_{i} \nabla^{i} \Phi+\theta R^{-1}\left(\frac{\rho}{R}\right)^{\alpha-\beta-1} \partial_{\rho} \Phi+\left(\frac{\rho}{R}\right)^{\alpha-\beta} \partial_{\rho}^{2} \Phi=0,
$$


donde $\nabla_{i}$ es la derivada covariante sobre la esfera $S^{p-4}$.

Proponemos la solución ${ }^{27}$

$$
\Phi^{\ell}=\phi^{\ell}(\rho) e^{i P \cdot y} Y^{\ell}\left(S^{p-4}\right),
$$

donde $Y^{\ell}\left(S^{p-4}\right)$ son los armónicos esféricos escalares de la $S^{p-4}$, que satisfacen la ecuación de autovalores

$$
\nabla^{i} \nabla_{i} Y^{\ell}\left(S^{p-4}\right)=-\ell(\ell+p-5) Y^{\ell}\left(S^{p-4}\right) .
$$

Reemplazando entonces la propuesta (6.4.10) en la EOM (6.4.9) resulta

$$
\begin{gathered}
\Phi_{i}^{\ell}=c_{i}\left(\frac{\rho}{R}\right)^{A-\gamma B} e^{i P \cdot y} Y^{\ell}\left(S^{p-4}\right) \\
\Phi_{X}^{\ell}=c_{X} s^{1 / 4} \Lambda^{-1 / 2}\left(\frac{\rho}{R}\right)^{A} J_{\gamma}\left[\frac{s^{1 / 2} R}{B}\left(\frac{\rho}{R}\right)^{-B}\right] e^{i P \cdot y} Y^{\ell}\left(S^{p-4}\right),
\end{gathered}
$$

donde, al igual que en las Secciones anteriores, hemos utilizado la solución completa para $\Phi$ en el segundo caso, correspondiente al estado intermedio $X$, y solamente el comportamiento dominante para la región $\rho \sim \rho_{i n t}$ para el estado hadrónico inicial y final $\Phi_{i}$. $J_{\gamma}$ es la función de Bessel de primera especie, y $s=-(P+q)^{2}=M_{X}^{2}$ es el cuadrado de la masa del estado intermedio, mientras que $c_{X}$ y $c_{i}$ son constantes adimensionales. El orden de esta función de Bessel viene dado por

$$
\gamma^{2}=\frac{A^{2}+\ell(\ell+p-5)}{B^{2}}
$$

donde definimos

$$
A=\frac{1-\theta}{2} \quad, \quad B=\frac{\alpha-\beta-2}{2} .
$$

Estos son mesones escalares y pseudoescalares para valores pares e impares de $\ell$ respectivamente. Puede verse fácilmente esto dado que la tranformación de paridad sobre los armónicos esféricos actúa como $\left[Y^{\ell}\left(S^{p-4}\right)\right]_{\mathrm{P}}=(-1)^{\ell} Y^{\ell}\left(S^{p-4}\right)$.

Aplicando el método descripto en la Sección 6.1 acoplamos estos mesones escalares holográficos a los campos de gauge en el interior del espacio. Esto lo hacemos considerando la fluctuación 6.1.11) en la métrica, y utilizando luego la ecuación de autovalores $v^{j} \partial_{j} Y^{\ell}(\Omega)=i \mathcal{Q}_{\ell} Y^{\ell}(\Omega)$, con lo que obtenemos el lagrangiano de interacción 28

$$
\mathcal{L}_{\text {interaction }}^{\text {scalar }}=i \mathcal{Q} \mu_{p}\left(\pi \alpha^{\prime}\right)^{2} \sqrt{-\operatorname{det} g}\left(\frac{\rho}{R}\right)^{\beta} A^{m}\left(\Phi \partial_{m} \Phi_{X}^{*}-\Phi_{X}^{*} \partial_{m} \Phi\right) .
$$

Tenemos entonces mesones que son autoestados de carga, con autovalor $\mathcal{Q}$ bajo el grupo de simetría $U(1)$, inducida por transformaciones en $S^{p-4}$ en la dirección del vector de Killing $v^{j}$.

También podemos deducir $\mathcal{L}_{\text {interaction }}^{\text {scalar }}$ como lo hicimos en la Sección 6.1, a partir del acoplamiento de un campo de gauge $A_{m}$ con la corriente de Noether correspondiente a las transformaciones globales que dejan invariante el lagrangiano (6.4.6). Estas son transformaciones de

\footnotetext{
${ }^{27}$ Esta solución será exacta para la métrica 6.4.2. Como esta es solo asintótica para los modelos considerados. i.e. D3D7, D4D8 $\overline{\mathrm{D} 8}$, y $\mathrm{D} 4 \mathrm{D} 6 \overline{\mathrm{D} 6}$, debemos asumir la condición $\rho_{\text {int }} \sim q R^{2} \gg \rho_{0} \equiv \Lambda R^{2}$ para el radio de interacción $\rho_{\text {int }}$, donde $\Lambda$ es el cutoff IR para la teoría de gauge tetradimensional.

${ }^{28}$ Aquí redefinimos $\Phi \equiv \Phi^{\ell}$ y $\mathcal{Q} \equiv \mathcal{Q}_{\ell}$, eliminando el subíndice $\ell$ de la notación, para no volverla engorrosa.
} 
$U(1) \subseteq S O(p-3)$, siendo este último el grupo de isometrías de $S^{p-4}{ }_{29}$ Esta corriente de Noether es

$$
j_{m}^{\text {scalar }}=i \mu_{p}\left(\pi \alpha^{\prime}\right)^{2}\left(\frac{\rho}{R}\right)^{\beta}\left(\Phi \partial_{m} \Phi_{X}^{*}-\Phi_{X}^{*} \partial_{m} \Phi\right),
$$

y podemos definir alternativamente $\mathcal{L}_{\text {interaction }}^{\text {scalar }}=\mathcal{Q} \sqrt{-\operatorname{det} g} A^{m} j_{m}^{\text {scalar }}$, recuperando el resultado de $\mathcal{L}_{\text {interaction. }}^{\text {scalar }}$ Notemos que los campos escalares de 6.4.10 poseen carga con respecto al grupo $U(1) \subseteq S O(p-3)$, con $\mathcal{Q}_{\ell} \neq 0$ para $\ell>0$.

Queremos aplicar ahora la prescripción holográfica dual (6.1.35) que relaciona la acción de interacción con el elemento de matriz con el que construiremos el tensor hadrónico

$$
S_{\text {interaction }}=(2 \pi)^{4} \delta^{4}\left(P_{X}-P-q\right) \tilde{n}_{\mu}\left\langle P+q, X\left|J^{\mu}(0)\right| P, \mathcal{Q}\right\rangle,
$$

donde $\tilde{n}_{\mu}$ es un vector de polarización unitario.

Para esto necesitamos en primer lugar encontrar la solución para el campo de gauge. Esta sale de la ecuación de Maxwell $D_{m} F^{m n}=0$ en las cinco dimensiones del $A d S_{5}$. Proponemos la forma

$$
\begin{aligned}
& A_{\mu}=\widetilde{n}_{\mu} e^{i q \cdot y} \hat{g}(\rho), \\
& A_{\rho}=e^{i q \cdot y} \hat{h}(\rho),
\end{aligned}
$$

que lleva implícita la elección de un gauge tipo Lorenz. Su solución es

$$
\begin{aligned}
& A_{\mu}=\widetilde{n}_{\mu} e^{i q \cdot y} \frac{1}{\Gamma(n+1)}\left(\frac{q R}{2 B}\right)^{n+1}\left(\frac{\rho}{R}\right)^{-(n+1) B} K_{n+1}\left[\frac{q R}{B}\left(\frac{\rho}{R}\right)^{-B}\right] \\
& A_{\rho}=-e^{i q \cdot y} \frac{i(q \cdot \widetilde{n})}{\Gamma(n+1)}\left(\frac{q R}{2 B}\right)^{n+1}\left(\frac{\rho}{R}\right)^{D} K_{n}\left[\frac{q R}{B}\left(\frac{\rho}{R}\right)^{-B}\right]=-\frac{i}{q^{2}} \eta^{\mu \nu} q_{\mu} \partial_{\rho} A_{\nu}
\end{aligned}
$$

con

$$
D=\frac{-4 \alpha+3 \beta+2}{4} \quad, \quad n=\frac{2+\beta}{4 B},
$$

y donde $B$ fue definido en 6.4 .15 . La ecuación para la conservación de la corriente $D_{a} j^{a}$ resulta $a^{30}$

$$
\partial \cdot j^{\text {scalar }}=\left(\frac{\rho}{R}\right)^{-\alpha-\frac{(p+3)}{2} \beta-(p-4)} \partial_{\rho}\left[\left(\frac{\rho}{R}\right)^{2 \alpha+\frac{(p-5)}{2} \beta+(p-4)} j_{\rho}^{\text {scalar }}\right],
$$

con lo que el acoplamiento es

$$
A_{m} j_{\text {scalar }}^{m}=\left(\frac{\rho}{R}\right)^{-\alpha} A_{\mu}\left[j_{\text {scalar }}^{\mu}-i \frac{q^{\mu}}{q^{2}}\left(\partial \cdot j_{\text {scalar }}\right)\right]-i \frac{q^{\nu}}{q^{2}}\left(\frac{\rho}{R}\right)^{3-\theta} \partial_{\rho}\left[\left(\frac{\rho}{R}\right)^{\theta-1} j_{\rho}^{\text {scalar }} A_{\nu}\right] .
$$

\footnotetext{
${ }^{29}$ En particular, para los dos modelos aquí estudiados, las isometrías de $S^{2}$ forman el grupo $S O(3)$, isomorfo a $S U(2) \supset U(1)$, mientras que las isometrías de $S^{4}$ forman $S O(5) \sim S O(2) \times S O(3)$, y $S O(3)$ se descompone como en el caso anterior [68. Puede demostrarse que vale esta inclusión para cualquier $4 \leq p \leq 9$.

${ }^{30}$ Notar que el subíndice $\rho$ se refiere simplemente a la variable $\rho$, por lo que su repetición no implica suma alguna.
} 
La acción de interacción resulta

$$
\begin{aligned}
S_{\text {interaction }}^{\text {scalar }}= & \mathcal{Q} \int_{\rho_{0}}^{\infty} d^{p+1} x \sqrt{-\operatorname{det} g} A_{m} j_{\text {scalar }}^{m} \\
= & \mathcal{Q} \int_{\rho_{0}}^{\infty} d^{p+1} x \sqrt{-\operatorname{det} g}\left(\frac{\rho}{R}\right)^{-\alpha} A_{\mu}\left[j_{\text {scalar }}^{\mu}-i \frac{q^{\mu}}{q^{2}}\left(\partial \cdot j_{\text {scalar }}\right)\right]+ \\
& \frac{q^{\nu}}{q^{2}} \mathcal{Q} \int_{\rho_{0}}^{\infty} d^{p+1} x \sqrt{-\operatorname{det} g}\left(\frac{\rho}{R}\right)^{-\theta} \partial_{\rho}\left[\left(\frac{\rho}{R}\right)^{\theta-1} j_{\rho}^{\text {scalar }} A_{\nu}\right] \\
\equiv & I_{1}^{\text {scalar }}+I_{2}^{\text {scalar }} .
\end{aligned}
$$

En el límite $\Lambda \ll q$ resulta $I_{2}^{\text {scalar }} \rightarrow 0$. Por otro lado, evaluendo $I_{1}^{\text {scalar }}$ y usando la prescripción (6.4.18) hallamos

$$
\begin{aligned}
\left\langle P+q, X\left|J^{\mu}(0)\right| P, \mathcal{Q}\right\rangle= & 2^{\gamma+2} B^{\gamma+1} \pi^{2} \frac{\Gamma(\gamma+n+2)}{\Gamma(n+1)} c_{i} c_{X}^{*} \mu_{p} \mathcal{Q} \alpha^{\prime 2}\left(s^{1 / 4} \Lambda^{-1 / 2}\right) \times \\
& \frac{q^{2 n+2} s^{\frac{\gamma}{2}} R^{p-\gamma-5}}{\left(q^{2}+s\right)^{2+\gamma+n}}\left(P^{\mu}+\frac{q^{\mu}}{2 x}\right) .
\end{aligned}
$$

Para $|t| \ll 1$ podemos aproximar $s \simeq q^{2}(1 / x-1)$, con lo que la expresión anterior queda

$$
\begin{aligned}
\left\langle P+q, X\left|J^{\mu}(0)\right| P, \mathcal{Q}\right\rangle= & 2^{\gamma+2} B^{\gamma+1} \pi^{2} \frac{\Gamma(\gamma+n+2)}{\Gamma(n+1)} c_{i} c_{X}^{*} \mu_{p} \mathcal{Q} \alpha^{2} R^{p-3} \times \\
& \left(\frac{\Lambda}{q}\right)^{\gamma+\frac{3}{2}} x^{\frac{\gamma}{2}+\frac{7}{4}+n}(1-x)^{\frac{\gamma}{2}+\frac{1}{4}}\left(P^{\mu}+\frac{q^{\mu}}{2 x}\right) .
\end{aligned}
$$

Con el mismo argumento que el utilizado en las Secciones anteriores calculamos $\operatorname{Im} T^{\mu \nu}$ multiplicando (6.4.26) por su complejo conjugado y sumando sobre sus excitaciones radiales. Estimamos la densidad de estados de la forma usual en este formalismo, imponiendo un cutoff IR en $\rho_{0} \equiv \Lambda R^{2}$. La distancia entre los ceros de la función de Bessel (6.4.13) para valores grandes del argumento es $M_{n^{\prime}}=n^{\prime} \pi \Lambda$, que en el límite de $N$ grande y para $q \gg \Lambda$ resulta

$$
\sum_{n^{\prime}} \delta\left(M_{n^{\prime}}^{2}-s\right) \sim\left(\frac{\partial M_{n^{\prime}}^{2}}{\partial n^{\prime}}\right)^{-1} \sim\left(2 \pi s^{1 / 2} \Lambda\right)^{-1} .
$$

Obtenemos pues

$$
\begin{aligned}
\operatorname{Im} T^{\mu \nu}= & 2^{2 \gamma+4} B^{2 \gamma+2} \pi^{5} \frac{\Gamma(\gamma+n+2)^{2}}{\Gamma(n+1)^{2}}\left|c_{i}\right|^{2}\left|c_{X}\right|^{2} \mu_{p}^{2} \mathcal{Q}^{2} \alpha^{4} R^{2 p-4} \times \\
& \left(\frac{\Lambda^{2}}{q^{2}}\right)^{\gamma+2} x^{\gamma+4+2 n}(1-x)^{\gamma}\left(P^{\mu}+\frac{q^{\mu}}{2 x}\right)\left(P^{\nu}+\frac{q^{\nu}}{2 x}\right) .
\end{aligned}
$$

Puede chequearse que nuestro $W^{\mu \nu}$ así calculado satisface los requisitos de simetría impuestos en el Capítulo 4 . De este calculamos las funciones de estructura para mesones escalares de 4.2.14),

$$
F_{1}=0, \quad F_{2}=A_{0}^{\text {scalar }} \mu_{p}^{2} \mathcal{Q}^{2} \alpha^{\prime 4} R^{2 p-6}\left(\frac{\Lambda^{2}}{q^{2}}\right)^{\gamma+1} x^{\gamma+3+2 n}(1-x)^{\gamma}
$$

donde definimos la constante adimensional $A_{0}^{\text {scalar }}=2^{2 \gamma+4} B^{2 \gamma+2} \pi^{5} \frac{\Gamma(\gamma+n+2)^{2}}{\Gamma(n+1)^{2}}\left|c_{i}\right|^{2}\left|c_{X}\right|^{2}$. Es fácil chequear que de esta solución general se recupera el resultado (6.2.26) para el modelo de D3D7branas 31

\footnotetext{
${ }^{31}$ Existe una diferencia en un factor adimensional $(\Lambda R)^{8}$, debida exclusivamente a las normalizaciones elegidas en cada caso para $c_{i}$ y $c_{X}$.
} 


\subsubsection{DIS desde mesones vectoriales para modelos más genera- les}

Calculamos aquí el tensor hadrónico para mesones vectoriales en el caso de una métrica general 6.4.2 para el caso de una sola $\mathrm{D} p$-brana de sabor $N_{f}=1$. En la próxima Sección calcularemos el caso con varias branas de sabor $N_{f}>1$ dando lugar a mesones vectoriales que transformen con respecto al grupo no abeliano $U\left(N_{f}\right)$ de simetría global. El cálculo es similar al estudiado en la Sección 6.3 .

Los mesones vectoriales provienen de fluctuaciones en los campos vectoriales de la acción de DBI sobre la $\mathrm{D} p$-brana, que ocurren en la dirección paralela al volumen de mundo de esta [12, 71, 72. Partimos de la acción (6.4.3) y calculamos las EOM para las fluctuaciones vectoriales solamente, manteniendo las escalares fijas $Z^{i}=$ cte, i.e. $\Phi=0$, y luego expandimos este lagrangiano a segundo orden en la fluctuación vectorial. Este resulta en las EOM

$$
\partial_{a}\left(\sqrt{-\operatorname{det} g} F^{a b}\right)=0
$$

donde $F^{a b}=\partial^{a} B^{b}-\partial^{b} B^{a}$, y los índices $a, b=0, \ldots, p$ recorren las direcciones del volumen de mundo de la D p-brana, consistente con la notación utilizada en toda la Tesis. Solamente consideramos el término de DBI en (6.4.3), pues el de Wess-Zumino no contribuye para las soluciones en las que estamos interesados. Expandiendo estas EOM obtenemos

$$
\square B^{\mu}-\partial^{\mu}(\partial \cdot B)+\theta R^{-1}\left(\frac{\rho}{R}\right)^{\alpha-\beta-1} \partial_{\rho} B^{\mu}+\left(\frac{\rho}{R}\right)^{\alpha-\beta} \partial_{\rho}^{2} B^{\mu}+\nabla_{i} \nabla^{i} B^{\mu}=0 .
$$

Proponemos el mismo Ansatz que utilizamos en la Sección 6.3 y que fue introducido en [12] para $B_{\mu}$, a saber

$$
B_{\mu}^{\ell}=\zeta_{\mu} \phi^{\ell}(\rho) e^{i P \cdot y} Y^{\ell}\left(S^{p-4}\right), \quad P \cdot \zeta=0, \quad B_{\rho}=0, \quad B_{i}=0,
$$

donde hemos expandido en $Y^{\ell}\left(S^{p-4}\right)$, armónicos esféricos sobre $S^{p-4}$, que satisfacen 6.4.11). Resta determinar la dependencia radial $\phi^{\ell}(\rho)$ y $\zeta_{\mu}$ es el vector polarización que cumple la condición de transversalidad $\zeta \cdot P=0$ proveniente de $\partial^{\mu} B_{\mu}=0$.

Insertando esta propuesta 6.4.32 en la ecuación 6.4.31) resulta

$$
\begin{gathered}
B_{\mu i}^{\ell}=\zeta_{\mu} \Lambda^{-1} c_{i}\left(\frac{\rho}{R}\right)^{A-\gamma B} e^{i P \cdot y} Y^{\ell}\left(S^{p-4}\right) \\
B_{\mu X}^{\ell}=\zeta_{\mu X} \Lambda^{-1} c_{X}\left(s^{-1 / 4} \Lambda^{1 / 2}\right)\left(\frac{\rho}{R}\right)^{A} J_{\gamma}\left[\frac{s^{1 / 2} R}{B}\left(\frac{\rho}{R}\right)^{-B}\right] e^{i P \cdot y} Y^{\ell}\left(S^{p-4}\right) .
\end{gathered}
$$

$\mathrm{Al}$ igual que en los casos anteriores, utilizamos la solución completa para $B_{\mu}^{\ell}$ en el caso del estado intermedio $X$ y la solución dominante asintóticamente en la región $\rho \sim \rho_{\text {int }}$ para el estado inicial y final $B_{\mu i}$. Nuevamente $J_{\gamma}$ es la función de Bessel de primera especie de orden $\gamma^{2}=\frac{A^{2}+\ell(\ell+p-5)}{B^{2}}$ y $s=-(P+q)^{2}=M_{X}^{2}$ es el cuadrado de la masa del estado intermedio, mientras que $c_{X}$ y $c_{i}$ son constantes adimensionales. Hemos utilizado las definiciones de $\theta, A$ y $B$ de las ecuaciones 6.4.8 y 6.4.15). Estas soluciones corresponden a mesones vectoriales para valores pares de $\ell$ y mesones axiales para valores impares de $\ell 32$

\footnotetext{
${ }^{32}$ Esto proviene, al igual que en el caso anterior, de la relación $\left[Y^{\ell}(\Omega)\right]_{\mathrm{P}}=(-1)^{\ell} Y^{\ell}(\Omega)$ y $\left[\zeta_{\mu}\right]_{\mathrm{P}}=$ $\left(\zeta^{0},-\vec{\zeta}\right)$.
} 
De la expansión de $B_{\mu}$ en armónicos esféricos de $S^{p-4}$ podemos ver que los campos de gauge sobre la $\mathrm{D} p$-brana corresponden a campos cargados en el $A d S_{5}$. Siguiendo un procedimiento análogo al de la Sección 6.3 vemos que los modos con $\ell=0$ corresponden a un campo de gauge abeliano $B_{\mu}^{0}$ en el espacio de cinco dimensiones. El resto de los campos vectoriales $B_{\mu}^{\ell}$ con $\ell>0$ son cargados y masivos en cuatro dimensiones, siendo $\mathcal{Q}_{\ell}$ sus cargas bajo $U(1) \subseteq S O(p-3)$ de la $S^{p-4}$ y $m_{\ell}^{2}=\ell(\ell+p-5) / R^{2}$ sus masas. Las EOM (6.4.31) para los mesones vectoriales en la región de interacción pueden derivarse también del lagrangiano cuadrátic ${ }^{33}$

$$
\mathcal{L}_{0}^{S F}=-\mu_{p}\left(\pi \alpha^{\prime}\right)^{2} \sqrt{-\operatorname{det} g} F^{a b} F_{a b}^{*} .
$$

Reproducimos la interacción en el interior del espacio de cinco dimensiones de la misma manera que en la Sección anterior, perturbando la métrica con la fluctuación (6.1.11). Volvemos a usar la ecuación de autovalores $v^{j} \partial_{j} Y^{\ell}(\Omega)=i \mathcal{Q}_{\ell} Y^{\ell}(\Omega)$ y obtenemos el lagrangiano de interacción

$$
\mathcal{L}_{\text {interaction }}^{S F}=i \mathcal{Q} \mu_{p}\left(\pi \alpha^{\prime}\right)^{2} \sqrt{-\operatorname{det} g} A_{m}\left[B_{X n}^{*} F^{n m}-B_{n}\left(F_{X}^{n m}\right)^{*}\right],
$$

donde $A_{m}$ es solución de Eq. 6.4.20). Al igual que en el caso anterior, podemos deducir este mismo lagrangiano a partir del acoplamiento del campo de gauge $A_{m}$ con la corriente de Noether de la acción libre 6.4.35, escribiendo $\mathcal{L}_{\text {interaction }}^{S F}=\mathcal{Q} \sqrt{-\operatorname{det} g} A_{m} j_{S F}^{m}$ donde

$$
j_{S F}^{m}=i \mu_{p}\left(\pi \alpha^{\prime}\right)^{2}\left[B_{X n}^{*} F^{n m}-B_{n}\left(F_{X}^{n m}\right)^{*}\right],
$$

es una corriente conservada. Resulta entonces una acción de interacción

$$
\begin{aligned}
S_{\text {interaction }}^{S F}= & \mathcal{Q} \int_{\rho_{0}}^{\infty} d^{p+1} x \sqrt{-\operatorname{det} g} A_{m} j_{S F}^{m} \\
= & \mathcal{Q} \int_{\rho_{0}}^{\infty} d^{p+1} x \sqrt{-\operatorname{det} g}\left(\frac{\rho}{R}\right)^{-\alpha} A_{\mu}\left[j_{S F}^{\mu}-i \frac{q^{\mu}}{q^{2}}\left(\partial \cdot j_{S F}\right)\right]+ \\
& \frac{q^{\nu}}{q^{2}} \mathcal{Q} \int_{\rho_{0}}^{\infty} d^{p+1} x \sqrt{-\operatorname{det} g}\left(\frac{\rho}{R}\right)^{-\theta} \partial_{\rho}\left[\left(\frac{\rho}{R}\right)^{\theta-1} j_{\rho}^{S F} A_{\nu}\right] \\
\equiv & I_{1}^{S F}+I_{2}^{S F} .
\end{aligned}
$$

Vemos que $I_{2}^{S F} \rightarrow 0$ para $\Lambda \ll q$. Evaluando $I_{1}^{S F}$ y utilizando la prescripción holográfica 6.4.18 encontramos

$$
\begin{aligned}
\left\langle P+q, X\left|J^{\mu}(0)\right| P, \mathcal{Q}\right\rangle= & 2^{\gamma} B^{\gamma+1} \pi^{2} \frac{\Gamma(\gamma+n+2)}{\Gamma(n+1)} c_{i} c_{X}^{*} \mu_{p} \mathcal{Q} \alpha^{\prime 2} R^{p-\gamma-5} \times \\
& \left(s^{-1 / 4} \Lambda^{1 / 2}\right) \frac{q^{2 \gamma+2} s^{\frac{\gamma}{2}}}{\left(q^{2}+s\right)^{2+\gamma+n}} N^{\mu}
\end{aligned}
$$

con

$$
N^{\mu}=2\left(\zeta \cdot \zeta_{X}\right)\left(P^{\mu}+\frac{q^{\mu}}{2 x}\right)+\left(\zeta_{X} \cdot q\right) \zeta^{\mu}-(\zeta \cdot q) \zeta_{X}^{\mu}
$$

Vemos fácilmente que $N^{\mu}$ contiene toda la información sobre la estructura tensorial del elemento de matriz 6.4.25 y por lo tanto las funciones de estructura dependerán solamente de este y serán independientes del modelo a menos de un factor global.

\footnotetext{
${ }^{33}$ Definimos a partir de aquí $B_{\mu}^{\ell} \equiv B_{\mu}$ para $\ell>0$, por simplicidad. El supraíndice $S F$ señala que estamos en presencia de mesones vectoriales con un solo sabor (Single Flavor, $N_{f}=1$ ), y se utiliza para diferenciarlo del caso con más de un sabor (supraíndice $M F$, por Multiple Flavor, $N_{f}>1$ ) que estudiaremos en la próxima Sección.
} 
Para $|t| \ll 1$ aproximamos $s \simeq q^{2}\left(\frac{1}{x}-1\right)$ obteniendo

$$
\begin{aligned}
\left\langle P+q, X\left|J^{\mu}(0)\right| P, \mathcal{Q}\right\rangle= & 2^{\gamma} B^{\gamma+1} \pi^{2} \frac{\Gamma(\gamma+n+2)}{\Gamma(n+1)} c_{i} c_{X}^{*} \mu_{p} \mathcal{Q} \alpha^{2} R^{p-3} \times \\
& \left(\frac{\Lambda}{q}\right)^{\gamma+\frac{5}{2}} x^{\frac{\gamma}{2}+\frac{9}{4}+n}(1-x)^{\frac{\gamma}{2}-\frac{1}{4}} N^{\mu} \\
\equiv & f_{\Lambda}^{(\gamma)}(x, q) N^{\mu} .
\end{aligned}
$$

Multiplicamos 6.4.42 por su complejo conjugado y sumamos sobre las excitaciones radiales y polarizaciones de los estado hadrónicos finales $\zeta_{X}^{\mu}$ para calcular $\operatorname{Im} T^{\mu \nu}$ a partir de 6.1.5). Estimamos la densidad de estados de la misma manera que para el caso de mesones escalares, obteniendo ahora

$$
\operatorname{Im} T^{\mu \nu}=\frac{\pi f f^{*}}{\Lambda s^{1 / 2}} \sum_{\lambda} N^{\mu} N^{* \nu}
$$

Normalizando las polarizaciones de los mesones vectoriales de manera que $\zeta^{\mu}\left(P_{X}, \lambda\right) \cdot \zeta_{\mu}^{*}\left(P_{X}, \lambda^{\prime}\right)=$ $-M_{X}^{2} \delta_{\lambda, \lambda^{\prime}}$ y despreciando los términos proporcionales a $q_{\mu}$ y $q_{\nu}$ como en el caso anterior, obtenemos

$$
\operatorname{Im} T_{\mu \nu} \equiv 2 \frac{\pi x^{\frac{1}{2}} f f^{*}}{\Lambda q(1-x)^{\frac{1}{2}}} H_{\mu \nu}=2 \frac{\pi x^{\frac{1}{2}} f f^{*}}{\Lambda q(1-x)^{\frac{1}{2}}}\left(H_{\mu \nu}^{S}+H_{\mu \nu}^{A}\right),
$$

donde $H_{\mu \nu}^{S}$ y $H_{\mu \nu}^{A}$ son las partes simétrica y antisimétrica de $H_{\mu \nu}$ respectivamente, definidas en 6.3 .26 y 6.3 .27 .

El cálculo del tensor $W_{\mu \nu}$ a partir de $\operatorname{Im} T_{\mu \nu}$ es trivial. Comparando este $W_{\mu \nu}$ así obtenido con la forma general (4.3.5) podemos extraer las ocho funciones de estructura luego de hacer unos simples cálculos algebraicos ${ }^{34}$

$$
\begin{aligned}
F_{1} & =A^{S F}(x) \frac{1}{12 x^{3}}\left(1-x-4 x^{2} t+4 x^{3} t\right) \\
F_{2} & =A^{S F}(x) \frac{1}{6 x^{3}}\left(1-x+12 x t-14 x^{2} t-12 x^{2} t^{2}\right) \\
b_{1} & =A^{S F}(x) \frac{1}{4 x^{3}}(1-x-x t) \\
b_{2} & =A^{S F}(x) \frac{1}{2 x^{3}}\left(1-x-x^{2} t\right) \\
b_{3} & =A^{S F}(x) \frac{1}{24 x^{3}}\left(1-4 x+8 x^{2} t\right) \\
b_{4} & =A^{S F}(x) \frac{1}{12 x^{3}}\left(-1+4 x-2 x^{2} t\right) \\
g_{1} & =A^{S F}(x) \frac{t}{8 x^{2}}(-7+6 x+8 x t), \\
g_{2} & =A^{S F}(x) \frac{1}{16 x^{4}}\left(3-3 x-4 x t+2 x^{2} t\right),
\end{aligned}
$$

con

$$
A^{S F}(x)=A_{0}^{S F} \mu_{p}^{2} \mathcal{Q}^{2} \alpha^{4} R^{2 p-6}\left(\frac{\Lambda^{2}}{q^{2}}\right)^{\gamma} x^{\gamma+2 n+5}(1-x)^{\gamma-1},
$$

donde $A_{0}^{S F}=(2 B)^{2 \gamma+2} \pi^{5} \frac{\Gamma(\gamma+n+2)^{2}}{\Gamma(n+1)^{2}}\left|c_{i}\right|^{2}\left|c_{X}\right|^{2}$ es una constante de normalización adimensional. Recordemos que $\gamma$ se define en (6.4.14) y $n$ en (6.4.21). Las constantes $\alpha$ y $\beta$ vienen de la

\footnotetext{
${ }^{34}$ Recordemos que siempre estamos en el régimen $|t|<<1$.
} 
definición de la métrica general (6.4.1), mientras que $A$ y $B$ están definidas en (6.4.15) y $\theta$ en 6.4.8.

Vemos que, al igual que con los mesones escalares, recuperamos los resultados para el caso D3D7 y pueden calcularse sencillamente los resultados para los sistemas D4D8 $\overline{\mathrm{D} 8}$ y D4D6 $\overline{\mathrm{D} 6}$.

En la última Sección del presente Capítulo se presentarán las relaciones entre las funciones de estructura que obtuvimos para los mesones vectoriales y pseudovectoriales polarizados considerados aquí.

\subsection{DIS desde mesones vectoriales con $N_{f}>1$}

Estudiaremos en esta Sección un caso algo más general que el estudiado en la anterior, permitiendo un valor para el número de $\mathrm{D} p$-branas mayor a uno, siempre en la aproximación de prueba $1<N_{f} \ll N$. Esto dará lugar a mesones vectoriales polarizados con $N_{f}>1$ sabores. Veremos que nuevamente las funciones de estructura para estos se descomponen en un factor dependiente y uno independiente del modelo respectivamente, al igual que sucedía en el caso con $N_{f}=1$. Calculamos analíticamente cada uno de los factores para un modelo general dado por la métrica inducida $(6.4 .2)$ sobre la $\mathrm{D} p$-brana. Todos los cálculos en esta Sección suceden en la aproximación a nivel árbol. En la siguiente Sección discutiremos las correcciones radiativas, que resultan en términos no dominantes en la expansión $\frac{1}{N}$. Los resultados de esta Sección se encuentran publicados en nuestro trabajo [2].

Consideramos el mismo background (6.4.1) que utilizamos en todo el Capítulo, con métrica inducida 6.4.2)

$$
d s^{2}=\left(\frac{\rho}{R}\right)^{\alpha} \eta_{\mu \nu} d x^{\mu} d x^{\nu}+\left(\frac{\rho}{R}\right)^{\beta}\left[d \rho^{2}+\rho^{2} d \Omega_{p-4}^{2}\right] .
$$

La diferencia radica en que ahora esta es la métrica inducida sobre las $N_{f} \mathrm{D} p$-branas, que siguen estando en la aproximación de prueba dado que $1<N_{f} \ll N$. Esta es la métrica en la región asintótica $\rho_{\text {int }} \gg \rho_{0}=\Lambda R^{2}$ de los modelos tratados.

Partimos de la acción de Dirac-Born-Infeld no abeliana expandida a primer orden [73]

$$
S_{0}^{M F}=-\mu_{p} \int d^{p+1} \xi \sqrt{-\operatorname{det} g}\left(\pi \alpha^{\prime}\right)^{2} \operatorname{Tr}\left(F^{2}\right),
$$

donde $F_{a b}=\partial_{a} B_{b}-\partial_{b} B_{a}+i\left[B_{a}, B_{b}\right]$ y $\mu_{p}=\left[(2 \pi)^{p} g_{s} \alpha^{\prime \frac{p+1}{2}}\right]^{-1}$. Esta es la generalización de 6.4.35 para el caso $N_{f}>1$.

Para calcular el tensor hadrónico utilizando la prescripción holográfica dual consideramos que el mesón se acopla a un campo de gauge (6.4.20) en el interior del modelo de supergravedad. Expandimos la acción (6.5.1) en términos de $B_{\mu}$ obteniendo ${ }^{35}$

$$
\mathcal{L}_{0}^{M F}=-\mu_{p}\left(\pi \alpha^{\prime}\right)^{2} \sqrt{-\operatorname{det} g} \operatorname{Tr}\left\{\widehat{F}_{a b}^{*} \widehat{F}^{a b}+\left(i \widehat{F}_{a b}^{*}\left[B^{a}, B^{b}\right]+\text { c.c. }\right)-\left[B_{a}^{*}, B_{b}^{*}\right]\left[B^{a}, B^{b}\right]\right\},(6.5
$$

donde hemos definido $\widehat{F}_{a b}=\partial_{a} B_{b}-\partial_{b} B_{a}=F_{a b}-i\left[B_{a}, B_{b}\right]$. Como veremos en la próxima Sección, los últimos dos términos son no dominantes con respecto al primero en la expansión $\frac{1}{N}$. Entonces, a primer orden solamente conservamos el primer término, lo que nos dará un lagrangiano de interacción igual al obtenido para el caso de $N_{f}=1$.

\footnotetext{
${ }^{35}$ Escribimos $\operatorname{Tr}\left(F_{a b}^{*} F^{a b}\right)$ en lugar de $\operatorname{Tr}\left(F_{a b} F^{a b}\right)$ pues sus EOM son las mismas.
} 
Las EOM pueden expandirse resultando

$$
\square B^{\mu}-\partial^{\mu}(\partial \cdot B)+\theta R^{-1}\left(\frac{\rho}{R}\right)^{\alpha-\beta-1} \partial_{\rho} B^{\mu}+\left(\frac{\rho}{R}\right)^{\alpha-\beta} \partial_{\rho}^{2} B^{\mu}+\nabla_{i} \nabla^{i} B^{\mu}=0,
$$

que son las mismas que 6.4.31). La propuesta para las soluciones es ahora

$$
\begin{aligned}
B_{\mu}^{\ell} & =\sum_{\mathcal{A}=1}^{N_{f}} B_{\mu}^{(\mathcal{A}) \ell} \tau_{\mathcal{A}}, \\
B_{\mu}^{(\mathcal{A}) \ell} & =\zeta_{\mu} c^{(\mathcal{A})} \phi^{\ell}(\rho) e^{i P \cdot y} Y^{\ell}\left(S^{(p-4)}\right), \quad P \cdot \zeta=0, \quad B_{\rho}^{(\mathcal{A}) \ell}=0, \quad B_{i}^{(\mathcal{A}) \ell}=0,
\end{aligned}
$$

donde $\tau_{\mathcal{A}}$ son los generadores del grupo de sabor $S U\left(N_{f}\right)$, que satisface el álgebra de Lie

$$
\left[\tau_{\mathcal{A}}, \tau_{\mathcal{B}}\right]=i f_{\mathcal{A B C}} \tau_{\mathcal{C}}
$$

También expandimos $B_{\mu}^{(\mathcal{A})}$ en armónicos esféricos $Y^{\ell}\left(S^{(p-4)}\right)$ que satisfacen Eq. 6.4.11). La dependencia radial $\phi(\rho)$ queda aún a determinar, $\zeta_{\mu}$ es el vector polarización que cumple $\zeta \cdot P=0$, proveniente de $\partial^{\mu} B_{\mu}=0$. Usando esta propuesta 6.5.4 en la ecuación (6.5.3 obtenemos una solución para cada componente $B_{\mu}^{(\mathcal{A})}$ que coincide con los mesones vectoriales con $N_{f}=1$ de la Sección anterior, a saber

$$
\begin{gathered}
B_{\mu i}^{(\mathcal{A}) \ell}=\zeta_{\mu} \Lambda^{-1} c_{i}^{(\mathcal{A})}\left(\frac{\rho}{R}\right)^{A-\gamma B} e^{i P \cdot y} Y^{\ell}\left(S^{(p-4)}\right) \\
B_{\mu X}^{(\mathcal{A}) \ell}=\zeta_{\mu X} \Lambda^{-1} c_{X}^{(\mathcal{A})}\left(s^{-1 / 4} \Lambda^{1 / 2}\right)\left(\frac{\rho}{R}\right)^{A} J_{\gamma}\left[\frac{s^{1 / 2} R}{B}\left(\frac{\rho}{R}\right)^{-B}\right] e^{i P \cdot y} Y^{\ell}\left(S^{(p-4)}\right) .
\end{gathered}
$$

Como siempre, utilizamos la solución completa para $B_{\mu}^{(\mathcal{A}) \ell}$ en el caso del estado intermedio $X$ y el comportamiento dominante en la región $\rho \sim \rho_{\text {int }}$ para el estado inicial y final. Como antes, $J_{\gamma}$ es la función de Bessel de primera especie de orden $\gamma^{2}=\frac{A^{2}+\ell(\ell+p-5)}{B^{2}}$ y $s=-(P+q)^{2}=M_{X}^{2}$ es el cuadrado de la masa del estado intermedio, siendo $c_{X}^{(\mathcal{A})}$ y $c_{i}^{(\mathcal{A})}$ constantes adimensionales. Utilizamos las definiciones para $\theta, A$ y $B$ de 6.4.8 y 6.4.15. De la expansión de $B_{\mu}^{(\mathcal{A})}$ en armónicos esféricos de $S^{(p-4)}$ vemos que los campos de gauge en las Dp-branas corresponden a campos cargados masivos para $\ell>1$ y tenemos un campo de gauge no masivo $B_{\mu}^{0}$ en el espacio de cinco dimensiones $0,1,2,3, \rho$.

Considerando la fluctuación de la métrica 6.1.11 y $v^{j} \partial_{j} Y^{\ell}(\Omega)=i \mathcal{Q}_{\ell} Y^{\ell}(\Omega)$ obtenemos el lagrangiano de interacción ${ }^{36}$

$$
\begin{aligned}
\mathcal{L}_{\text {interaction }}^{M F}= & i \mathcal{Q} \mu_{p}\left(\pi \alpha^{\prime}\right)^{2} \sqrt{-\operatorname{det} g}\left\{A_{m} \operatorname{Tr}\left(B_{X n}^{*} \widehat{F}^{n m}-B_{n}\left(\widehat{F}_{X}^{n m}\right)^{*}\right)+\right. \\
& \left.i A_{m} \operatorname{Tr}\left(B_{n}\left[B_{X}^{m *}, B_{X}^{n *}\right]\right)+i A_{m} \operatorname{Tr}\left(B_{X n}^{*}\left[B^{m}, B^{n}\right]\right)\right\} \\
\equiv & \mathcal{L}_{\text {interaction }_{1}}^{M F}+\mathcal{L}_{\text {interaction }_{2}}^{M F}+\mathcal{L}_{\text {interaction }_{3}}^{M F} .
\end{aligned}
$$

Vemos aquí que el término $\mathcal{L}_{\text {interaction }}^{M F}$ no contribuye al proceso de interés, pues involucra dos estados iniciales para el hadrón $B_{\mu}$. Por otro lado, veremos en la próxima Sección que el término $\mathcal{L}_{\text {interaction }_{2}}^{M F}$ contribuye solamente a los diagramas no dominantes en la expansión $\frac{1}{N}$. Por lo tanto,

\footnotetext{
${ }^{36}$ En adelante definimos $B_{\mu} \equiv B_{\mu}^{\ell}$, omitiendo el índice $\ell$. El supraíndice $M F$ indica que son mesones con más de un sabor (Multi Flavored), es decir con $N_{f}>1$.
} 
solo nos quedamos con el orden dominante para el proceso de la Figura 6.1, que involucra el primer término $\mathcal{L}_{\text {interaction }_{1}}^{M F}$. Este es el mismo diagrama presente en el caso de mesones vectoriales con $N_{f}=1$ estudiado en la Sección anterior y vuelve a dibujarse en la Figura 6.3. Podemos verlo nuevamente como el acoplamiento del campo de gauge $A_{m}$ a una cierta corriente $j_{M F}^{m}$. Por lo tanto, $\mathcal{L}_{\text {interaction }_{1}}^{M F}=\mathcal{Q} \sqrt{-\operatorname{det} g} A_{m} j_{M F}^{m}$ donde

$$
j_{M F}^{m}=i \mu_{p}\left(\pi \alpha^{\prime}\right)^{2} \operatorname{Tr}\left(B_{X n}^{*} F^{n m}-B_{n}\left(F_{X}^{n m}\right)^{*}\right) .
$$

La acción de interacción es

$$
\begin{aligned}
S_{\text {interaction }}^{M F}= & \mathcal{Q} \int_{\rho_{0}}^{\infty} d^{p+1} x \sqrt{-\operatorname{det} g} A_{m} j_{M F}^{m} \\
= & \mathcal{Q} \int_{\rho_{0}}^{\infty} d^{p+1} x \sqrt{-\operatorname{det} g}\left(\frac{\rho}{R}\right)^{-\alpha} A_{\mu}\left[j_{M F}^{\mu}-i \frac{q^{\mu}}{q^{2}}\left(\partial \cdot j_{M F}\right)\right]+ \\
& \frac{q^{\nu}}{q^{2}} \mathcal{Q} \int_{\rho_{0}}^{\infty} d^{p+1} x \sqrt{-\operatorname{det} g}\left(\frac{\rho}{R}\right)^{-\theta} \partial_{\rho}\left[\left(\frac{\rho}{R}\right)^{\theta-1} j_{\rho}^{M F} A_{\nu}\right] \\
\equiv & I_{1}^{M F}+I_{2}^{M F} .
\end{aligned}
$$

Como vimos anteriormente, $I_{2}^{M F}=I_{2}^{S F} \rightarrow 0$ en nuestro límite de interés $\Lambda \ll q$. Evaluando $I_{1}^{M F}$ resulta

$$
\begin{aligned}
\left\langle P+q, X\left|J^{\mu}(0)\right| P, \mathcal{Q}\right\rangle= & C_{f} \delta_{\mathcal{A B}} I_{1}^{S F} \\
= & C_{f} \delta_{\mathcal{A B}}(2 B)^{\gamma+2} \pi^{2} \frac{\Gamma(\gamma+n+2)}{\Gamma(n+1)} c_{i} c_{X}^{*} \mu_{p} \mathcal{Q} \alpha^{2} R^{p-\gamma-5} \\
& \left(s^{-1 / 4} \Lambda^{1 / 2}\right) \frac{q^{2 \gamma+2} s^{\frac{\gamma}{2}-n}}{\left(q^{2}+s\right)^{2+\gamma+n}} N^{\mu} \\
= & C_{f} \delta_{\mathcal{A B}} f_{\Lambda}^{(\gamma)}(x, q) N^{\mu}
\end{aligned}
$$

donde definimos $f_{\Lambda}^{(\gamma)}(x, q)$ como en la Sección anterior y utilizamos

$$
\begin{array}{rlrl}
B_{\mu i} & =B_{\mu i}^{(\mathcal{A})} \tau_{\mathcal{A}}, & & \text { (no hay suma) } \\
B_{\mu X}=B_{\mu X}^{(\mathcal{B})} \tau_{\mathcal{B}}, & & \text { (no hay suma) }
\end{array}
$$

y

$$
N^{\mu}=2\left(\zeta \cdot \zeta_{X}\right)\left(P^{\mu}+\frac{q^{\mu}}{2 x}\right)+\left(\zeta_{X} \cdot q\right) \zeta^{\mu}-(\zeta \cdot q) \zeta_{X}^{\mu}
$$

También usamos

$$
\left[\tau_{\mathcal{A}}, \tau_{\mathcal{B}}\right]=i f_{\mathcal{A B C}} \tau_{\mathcal{C}} \quad, \quad \operatorname{Tr}\left(\tau_{\mathcal{A}} \tau_{\mathcal{B}}\right)=C_{f} \delta_{\mathcal{A B}}
$$

donde $C_{f}$ es el Casimir de $S U\left(N_{f}\right)$ y vale $C_{f}=\frac{1}{2}$ para la representación fundamental.

Para $|t| \ll 1$ aproximamos $s \simeq q^{2}\left(\frac{1}{x}-1\right)$ y entonces

$$
\begin{aligned}
\left\langle P+q, X\left|J^{\mu}(0)\right| P, \mathcal{Q}\right\rangle= & C_{f} \delta_{A B} \mathcal{Q} \mu_{p}(2)^{\gamma} B^{\gamma+1} \frac{\Gamma(\gamma+n+2)}{\Gamma(n+1)}\left(\pi \alpha^{\prime}\right)^{2} c_{i} c_{X}^{*} N^{\mu} R^{p-3} \\
& \times\left(\frac{\Lambda}{q}\right)^{\gamma+\frac{5}{2}} x^{\frac{\gamma}{2}+n+\frac{9}{4}}(1-x)^{\frac{\gamma}{2}-\frac{1}{4}}
\end{aligned}
$$


Para obtener Im $T^{\mu \nu}$ multiplicamos 6.5 .15 por su complejo conjugado y sumamos sobre las excitaciones radiales y polarizaciones del estado intermedio $\zeta_{X}^{\mu}$. La densidad de estados se estima de la manera usual y eliminamos los términos proporcionales a $q_{\mu}$ y $q_{\nu}$ obteniendo

$$
\operatorname{Im} T^{\mu \nu}=C_{f}^{2} \delta_{\mathcal{A} \mathcal{B}_{\mathcal{X}}} \frac{\pi f f^{*}}{\Lambda s^{1 / 2}} \sum_{\lambda} N^{\mu} N^{* \nu}=C_{f}^{2} \delta_{\mathcal{A} \mathcal{B}_{\mathcal{X}}} \frac{\pi x^{\frac{1}{2}} f f^{*}}{\Lambda q(1-x)^{\frac{1}{2}}}\left(H_{\mu \nu}^{S}+H_{\mu \nu}^{A}\right),
$$

donde definimos $\left\langle P+q, X\left|J^{\mu}(0)\right| P, \mathcal{Q}\right\rangle=C_{f} \delta_{\mathcal{A} \mathcal{B}_{X}} f_{\Lambda}^{(\gamma)}(x, q) N^{\mu}$, donde $H_{\mu \nu}^{S}$ y $H_{\mu \nu}^{A}$ son exactamente los mismos de 6.3.26 y 6.3.27).

Reescribiendo el tensor hadrónico $W_{\mu \nu}$ desde 4.3.5), obtenemos

$$
\begin{aligned}
F_{1} & =A^{M F}(x) \frac{1}{12 x^{3}}\left(1-x-4 x^{2} t+4 x^{3} t\right), \\
F_{2} & =A^{M F}(x) \frac{1}{6 x^{3}}\left(1-x+12 x t-14 x^{2} t-12 x^{2} t^{2}\right), \\
b_{1} & =A^{M F}(x) \frac{1}{4 x^{3}}(1-x-x t), \\
b_{2} & =A^{M F}(x) \frac{1}{2 x^{3}}\left(1-x-x^{2} t\right), \\
b_{3} & =A^{M F}(x) \frac{1}{24 x^{3}}\left(1-4 x+8 x^{2} t\right), \\
b_{4} & =A^{M F}(x) \frac{1}{12 x^{3}}\left(-1+4 x-2 x^{2} t\right), \\
g_{1} & =A^{M F}(x) \frac{t}{8 x^{2}}(-7+6 x+8 x t), \\
g_{2} & =A^{M F}(x) \frac{1}{16 x^{4}}\left(3-3 x-4 x t+2 x^{2} t\right),
\end{aligned}
$$

donde

$$
A^{M F}(x)=A_{0}^{M F} \mu_{p}^{2} \mathcal{Q}^{2} \alpha^{4} R^{2 p-6}\left(\frac{\Lambda^{2}}{q^{2}}\right)^{\gamma} x^{\gamma+2 n+5}(1-x)^{\gamma-1},
$$

y $A_{0}^{M F}=2 C_{f}^{2} \delta_{\mathcal{A} \mathcal{B}_{X}}(2 B)^{2 \gamma+2} \pi^{5} \frac{\Gamma(\gamma+n+2)^{2}}{\Gamma(n+1)^{2}}\left|c_{i}^{(\mathcal{A})}\right|^{2}\left|c_{X}^{\left(\mathcal{B}_{X}\right)}\right|^{2}$ es una constante adimensional. El subíndice $\mathcal{A}$ indica el sabor del mesón incidente y $\mathcal{B}_{X}$ el del estado intermedio. Todas estas ecuaciones se obtuvieron en el límite $|t| \ll 1[2]$.

Notemos que si tomamos el límite del caso abeliano (con un solo sabor) obtenemos el mismo conjunto de funciones de estructura calculados en la Sección anterior ${ }^{37}$. Con esto, podemos resumir los resultados aquí obtenidos como

$$
F_{i}^{(a) M F}(x, t)=C_{f}^{2} \delta_{\mathcal{A} \mathcal{B}_{X}} F_{i}^{(a) S F}(x, t),
$$

donde (a) indica un dado modelo holográfico e $i$ cada una de las funciones de estructura $i=$ $1, \cdots, 8$.

Por otro lado, para cada par de modelos holográficos duales $(a)$ y $(b)$ encontramos la relación $F_{i}^{(a)}(x, t)=A_{(a, b)}(x) F_{i}^{(b)}(x, t)$, que implica la relación entre los tensores hadrónicos

$$
W_{(a)}^{\mu \nu}=A_{(a, b)}(x) W_{(b)}^{\mu \nu} .
$$

Además, encontramos relaciones entre las diferentes funciones de estructura válidas para todos los modelos. Esto lo discutiremos en la Sección 6.7.

\footnotetext{
${ }^{37}$ Podemos redefinir las constantes de normalización como $c_{i}^{(A)}=c_{i} / \sqrt{C_{f}}$ y $c_{X}^{\left(\mathcal{B}_{X}\right)}=c_{X} / \sqrt{C_{f}}$ para el caso abeliano.
} 


\subsection{Contribuciones subdominantes en las expansio- nes $\frac{1}{N} \mathbf{y} \frac{N_{f}}{N}$}

En esta Sección investigamos las contribuciones no dominantes en las expansiones $1 / N$ y $N_{f} / N$ de las funciones de correlación de dos puntos de corrientes de simetría globales. Explicamos por qué en el límite planar solo tenemos que preocuparnos por el diagrama de Witten a nivel árbol de la Figura 6.1, que es el dual holográfico al diagrama de Feynman para la dispersión Compton de un leptón cargado por un hadrón. Consideramos los lagrangianos completos de los casos estudiados en las Secciones 6.4.1, 6.4.2 y 6.5, correspondientes a mesones escalares y vectoriales con $N_{f}=1$ y mesones vectoriales con $N_{f}>1$ respectivamente. Estudiamos las contribuciones no dominantes que surgen de los diagramas a un loop.

\section{Reducción a cinco dimensiones de SUGRA del tipo IIB}

Revisamos rápidamente la reducción a cinco dimensiones de la supergravedad ( $S U G R A$ ) tipo IIB en una $S^{5}$ siguiendo los pasos de [74 $]^{38}$. Esto es para dar un ejemplo de conteo de potencias de $N$ para diagramas de Feynman en SUGRA.

Comenzamos con la acción de SUGRA IIB en $D=10$ dimensiones escrita en el marco de Einstein (Einstein frame). Esta contiene el gravitón, el dilatón $\phi$, el axión de Ramond-Ramond $\mathcal{C}$ y la 5 -forma $F_{5}$

$$
S_{I I B}^{S U G R A}=-\frac{1}{2 \kappa_{10}^{2}} \int d^{10} x \sqrt{|\operatorname{det} g|}\left[\mathcal{R}_{10}-\frac{1}{2}(\partial \phi)^{2}-\frac{1}{2} e^{2 \phi}(\partial \mathcal{C})^{2}-\frac{1}{4 \cdot 5 !}\left(F_{5}\right)^{2}\right],
$$

donde $2 \kappa_{10}(2 \pi)^{7} g_{s}^{2} \alpha^{4}$. Consideramos un espacio $A d S_{5} \times S^{5}$ de radio $R^{4}=4 \pi g_{s} N \alpha^{2}$.

La acción reducida a cinco dimensiones nos da, en términos del dilatón 5-dimensional $\phi_{5}(x)$,

$$
S_{5 d}^{S U G R A}=-\frac{1}{2 \kappa_{5}^{2}} \int d^{5} x \sqrt{\left|\operatorname{det} g_{5}\right|}\left[\mathcal{R}_{5}-\frac{1}{2}\left(\partial \phi_{5}\right)^{2}+\cdots\right],
$$

donde los puntos suspensivos indican términos que no son relevantes a esta discusión. Definimos la constante de Newton en cinco dimensiones $\kappa_{5}$

$$
\frac{1}{2 \kappa_{5}^{2}}=\frac{N^{2}}{8 \pi^{2}}
$$

Vemos aquí la forma en que aparece el factor $N^{2}$ en la acción 5-dimensional. Al considerar un caso con $N_{f}$ branas de sabor, que será el sistema de D3D7-branas para fijar ideas, el conteo de potencias en la acción de SUGRA sumada a la acción de DBI es de la forma

$$
S=N^{2}\left[\tilde{S}_{I I B}^{S U G R A}+\frac{N_{f}}{N} \tilde{S}_{D B I}\right]
$$

donde con $\tilde{S}$ notamos las acciones cuyos términos no dependen de $N$. Entonces, para obtener campos canónicamente normalizados tenemos que redefinir el dilatón 5-dimensional como $\tilde{\phi}_{5} \equiv N \phi_{5}$, y hacer una redefinición análoga para el gravitón. Con los campos normalizados de esta forma en la acción $S$ se obtiene el conteo correcto en potencias de $N$ en cada vértice de interacción. Entonces, podemos construir los diagramas de Witten para procesos duales holográficos mostrando el conteo de potencias en $N$ correspondiente en cada caso.

\footnotetext{
${ }^{38}$ También pueden consultarse las referencias $[75,76,77$,
} 


\section{Correcciones $\frac{1}{N}$ para mesones escalares}

La parte relevante del lagrangiano para mesones escalares 6.4 .6 se puede escribir 39

$$
\mathcal{L}_{0}^{\text {scalar }}=-\mu_{p}\left(2 \pi \alpha^{\prime}\right)^{2} \sqrt{-\operatorname{det} g}\left(\frac{\rho}{R}\right)^{\beta} \frac{1}{2} g^{a b} \partial_{a} \Phi \partial_{b} \Phi^{*} .
$$

Factorizando el campo escalar ${ }^{40}$

$$
\Phi\left(\rho, y^{\mu}, \Omega\right)=\sum_{\ell} \varphi^{\ell}\left(\rho, y^{\mu}\right) Y^{\ell}(\Omega)
$$

y definiendo

$$
\sqrt{-\operatorname{det} g^{5}}=\left(\frac{\rho}{R}\right)^{\alpha+\frac{\beta}{2}}
$$

podemos escribir la acción libre en cinco dimensiones para cada $\varphi^{\ell}$ como

$$
S_{0}^{\text {scalar }}=-\mu_{p}\left(\pi \alpha^{\prime}\right)^{2} R^{p-4} \int d^{5} x \sqrt{-\operatorname{det} g^{5}}\left(\frac{\rho}{R}\right)^{(\alpha+\beta)+(p-4)\left(1+\frac{\beta}{2}\right)}\left[\partial^{m} \varphi^{\ell} \partial_{m} \varphi^{\ell *}+M_{\ell}^{2} \varphi^{\ell} \varphi^{\ell *}\right],
$$

donde usamos

$$
\int d^{p-4} \Omega \sqrt{\operatorname{det} \widetilde{g}} Y^{\ell} Y^{\ell^{\prime} *}=\delta_{\ell \ell^{\prime}}
$$

y definimos la normalización

$$
\int d^{p-4} \Omega \sqrt{\operatorname{det} \tilde{g}} \eta^{i j} \partial_{i} Y^{\ell} \partial_{j} Y^{\ell^{\prime} *} \equiv \delta_{\ell \ell^{\prime}} M_{\ell}^{2}
$$

Notar que 6.6.8 es la acción para un campo escalar complejo ${ }^{41}$ El lagrangiano de interacción era 6.2 .14

$$
\mathcal{L}_{\text {interaction }}^{\text {scalar }}=i \mathcal{Q} \mu_{p}\left(\pi \alpha^{\prime}\right)^{2} \sqrt{-\operatorname{det} g}\left(\frac{\rho}{R}\right)^{\beta} A^{m}\left(\Phi \partial_{m} \Phi^{*}-\Phi^{*} \partial_{m} \Phi\right),
$$

que bajo la reducción dimensional se escrib€ 42

$$
S_{\text {interaction }}^{\text {scalar }}=i \mathcal{Q} \mu_{p}\left(\pi \alpha^{\prime}\right)^{2} R^{p-4} \int d^{5} x \sqrt{-\operatorname{det} g^{5}}\left(\frac{\rho}{R}\right)^{(\alpha+\beta)+(p-4)\left(1+\frac{\beta}{2}\right)} A^{m}\left[\varphi \partial_{m} \varphi^{*}-\varphi^{*} \partial_{m} \varphi\right] .
$$

$\mathrm{Al}$ orden al que estamos interesados son relevantes las perturbaciones de la métrica de la forma

$$
g_{m n} \rightarrow g_{m n}+h_{m n},
$$

\footnotetext{
${ }^{39}$ Excluimos el primer término en $(6.4 .6$ pues no contribuye a la dinámica de los mesones.

${ }^{40}$ Notar que esta factorización no es la misma que en 6.4.10, pues aquí incluimos la dependencia radial en el campo $\varphi^{\ell}$.

${ }^{41}$ Estamos utilizando siempre la signatura $(-,+,+,+,+)$, lo que nos da el signo correcto para la masa en la acción. La acción completa para el escalar libre es $S_{0}^{\text {scalar }}=\sum_{\ell} S_{0}^{\text {scalar } \ell}$, y estamos escribiendo solamente una $S_{0}^{\text {scalar } \ell}$ en $(6.6 .8)$.

${ }^{42}$ En la última ecuación no escribimos el subíndice $X$ en los lagrangianos de interacción, convención que mantendremos en lo que resta de esta Sección. También dejamos de escribir el índice $\ell$.
} 
que corresponden a gravitones. Estos son gravitones genéricos y no necesariamente tienen la forma descripta en 6.1.11). Esta fluctuación induce los términos de interacción ${ }^{43}$

$$
h^{m n} \partial_{m} \Phi \partial_{n} \Phi^{*} \quad ; \quad h^{m n} A_{m} \Phi \partial_{n} \Phi^{*},
$$

que, bajo la reducción a cinco dimensiones en (6.6.8), resulta

$$
h^{m n} \partial_{m} \varphi \partial_{n} \varphi^{*} \quad, \quad h^{m n} A_{m} \varphi \partial_{n} \varphi^{*} .
$$

Uniendo todos los factores resulta la acción 5-dimensional completa

$$
\begin{aligned}
S_{\text {total }}^{\text {scalar }}= & S_{0}^{\text {scalar }}+S_{\text {interaction }}^{\text {scalar }} \\
= & -\mu_{p}\left(\pi \alpha^{\prime}\right)^{2} R^{p-4} \int d^{5} x \sqrt{-\operatorname{det} g^{5}}\left(\frac{\rho}{R}\right)^{(\alpha+\beta)+(p-4)\left(1+\frac{\beta}{2}\right)}\left[\partial^{m} \varphi \partial_{m} \varphi^{*}+M_{\ell}^{2} \varphi \varphi^{*}+\right. \\
& \left.h^{m n} \partial_{m} \varphi \partial_{n} \varphi^{*}-i \mathcal{Q} A^{m}\left(\varphi \partial_{m} \varphi^{*}-\varphi^{*} \partial_{m} \varphi\right)-i \mathcal{Q} h^{m n} A_{m}\left(\varphi \partial_{n} \varphi^{*}-\varphi^{*} \partial_{n} \varphi\right)\right],
\end{aligned}
$$

que incluye el término cinético para los mesones escalares, así como los términos de interacción con el gravitón y el campo de gauge $A_{m}$. Los últimos dos términos pueden verse como parte de una derivada covariante en el término cinético del mesón escalar. Podemos redefinir ahora los campos para que queden canónicamente normalizados

$$
\begin{aligned}
\widetilde{\varphi} & \equiv \sqrt{N} \varphi, \\
\widetilde{A}^{m} & \equiv N A^{m}, \\
\widetilde{h}^{m n} & \equiv N h^{m n} .
\end{aligned}
$$

Teniendo en cuenta que

$$
\mu_{p}\left(\pi \alpha^{\prime}\right)^{2}=\frac{\left(\pi \alpha^{\prime}\right)^{2}}{(2 \pi)^{p} g_{s} \alpha^{\prime \frac{p+1}{2}}}=\frac{1}{2^{p} \pi^{p-2}} \frac{N}{\lambda \alpha^{\prime \frac{p-3}{2}}}
$$

podemos escribir $S_{\text {total }}^{\text {scalar }}$ explícitamente en términos de potencias de $N$ como

$$
\begin{aligned}
S_{\text {total }}^{\text {scalar }}= & \frac{R^{p-4}}{2^{p} \pi^{p-2} \lambda \alpha^{\prime \frac{p-3}{2}}} \int d^{5} x \sqrt{-\operatorname{det} g^{5}}\left(\frac{\rho}{R}\right)^{(\alpha+\beta)+(p-4)\left(1+\frac{\beta}{2}\right)} \\
& {\left[\partial^{m} \widetilde{\varphi} \partial_{m} \widetilde{\varphi}^{*}+M_{\ell}^{2} \widetilde{\varphi} \widetilde{\varphi}^{*}+N^{-1} \widetilde{h}^{m n} \partial_{m} \widetilde{\varphi} \partial_{n} \widetilde{\varphi}^{*}-\right.} \\
& \left.N^{-1} i \mathcal{Q} \widetilde{A}^{m}\left(\widetilde{\varphi} \partial_{m} \widetilde{\varphi}^{*}-\widetilde{\varphi}^{*} \partial_{m} \widetilde{\varphi}\right)-N^{-2} i \mathcal{Q} \widetilde{h}^{m n} \widetilde{A_{m}}\left(\widetilde{\varphi} \partial_{n} \widetilde{\varphi}^{*}-\widetilde{\varphi}^{*} \partial_{n} \widetilde{\varphi}\right)\right]
\end{aligned}
$$

Con esto podemos construir los diagramas relevantes a nuestro proceso como se ve en las Figuras 6.3 y 6.4 . Vemos que solo el diagrama a orden árbol de la Figura 6.3 contribuye al orden dominante en $N$, a saber $N^{-2}$, mientras que los diagramas de la Fiugra 6.4 son no dominantes, de orden $N^{-4}$.

\footnotetext{
${ }^{43} \mathrm{El}$ término cinético del gravitón, así como el del campo de gauge $A^{m}$ vienen de la acción de SUGRA 10-dimensional discutida en la Subsección anterior. Aquí solo consideramos la $S_{D B I}$ discutida en las Secciones 6.4.1, 6.4.2 y 6.5, definida sobre el volumen de mundo de las $\mathrm{D} p$-branas.
} 


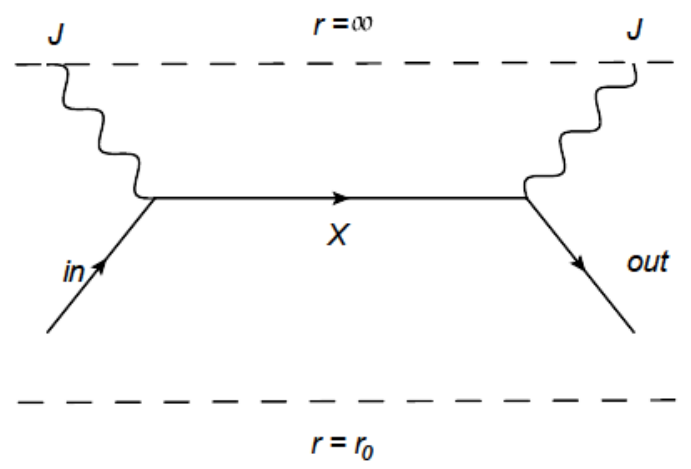

Figura 6.3: Diagrama de Witten 5-dimensional a nivel árbol, que representa la versión holográfica dual de la dispersión Compton de un leptón cargado por un hadrón en cuatro dimensiones. Este diagrama está compuesto por campos en cinco dimensiones obtenidos por la reducción dimensional de SUGRA 10-dimensional en una variedad de Einstein compacta. El campo dual al hadrón está indicado por una línea contínua; este hadrón puede ser un escalar o un vector con $N_{f}=1$ o $N_{f}>1$ según el caso que se desee estudiar. Las líneas onduladas indican el campo dual correspondiende a un fotón virtual intercambiado entre el leptón (no dibujado en este diagrama) y el hadrón. Este campo dual al fotón virtual es una fluctuación $A_{\mu}$ inducida por la inserción del operador de una corriente de simetría global $J_{\mu}$ en la teoría del borde. Este diagrama de Witten da la contribución dominante en la expansión $1 / N$. Notar que $r \rightarrow \infty$ corresponde al borde, donde está definida la teoría de gauge 4-dimensional y donde se insertan los operadores de corriente $J$.

\section{Correcciones $\frac{1}{N}$ para mesones vectoriales con $N_{f}=1$}

Consideramos la parte relevante del lagrangiano libre para mesones vectoriales con $N_{f}=1$ dado por 6.3.16

$$
\mathcal{L}_{0}^{S F}=-\mu_{p}\left(\pi \alpha^{\prime}\right)^{2} \sqrt{-\operatorname{det} g} F^{a b} F_{a b}^{*},
$$

y definimos

$$
\begin{aligned}
B^{a}\left(\rho, y^{\mu}, \Omega\right) & =\sum_{\ell} b^{a \ell}\left(\rho, y^{\mu}\right) Y^{\ell}(\Omega), \\
f_{m n}^{\ell} & =\partial_{m} b_{n}^{\ell}-\partial_{n} b_{m}^{\ell} .
\end{aligned}
$$

Podemos entonces escribir la acción libre reducida a cinco dimensiones para cada $b_{n}^{\ell}$ com 44

$$
S_{0}^{S F}=-\mu_{p}\left(2 \pi \alpha^{\prime}\right)^{2} R^{p-4} \int d^{5} x \sqrt{-\operatorname{det} g^{5}}\left(\frac{\rho}{R}\right)^{(\alpha+\beta)+(p-4)\left(1+\frac{\beta}{2}\right)}\left[\frac{1}{4} f^{m n} f_{m n}^{*}+\frac{1}{2} M_{\ell}^{2} b^{m} b_{m}^{*}\right],
$$

que es un lagrangiano del cual se derivan EOM del tipo Proca, correspondiente a un campo vectorial masivo.

Luego de la reducción a cinco dimensiones, el lagrangiano de interacción (6.3.17)

$$
\mathcal{L}_{\text {interaction }}^{S F}=i \mathcal{Q} \mu_{p}\left(\pi \alpha^{\prime}\right)^{2} \sqrt{-\operatorname{det} g} A_{m}\left[B_{n}^{*} F^{n m}-B_{n}\left(F^{n m}\right)^{*}\right],
$$

\footnotetext{
${ }^{44} \mathrm{~A}$ partir de ahora y hasta el final de esta Subsección omitimos el índice $\ell$. La ecuación que sigue es la parte de la acción correspondiente a cada uno de los $b_{n}^{\ell}$.
} 

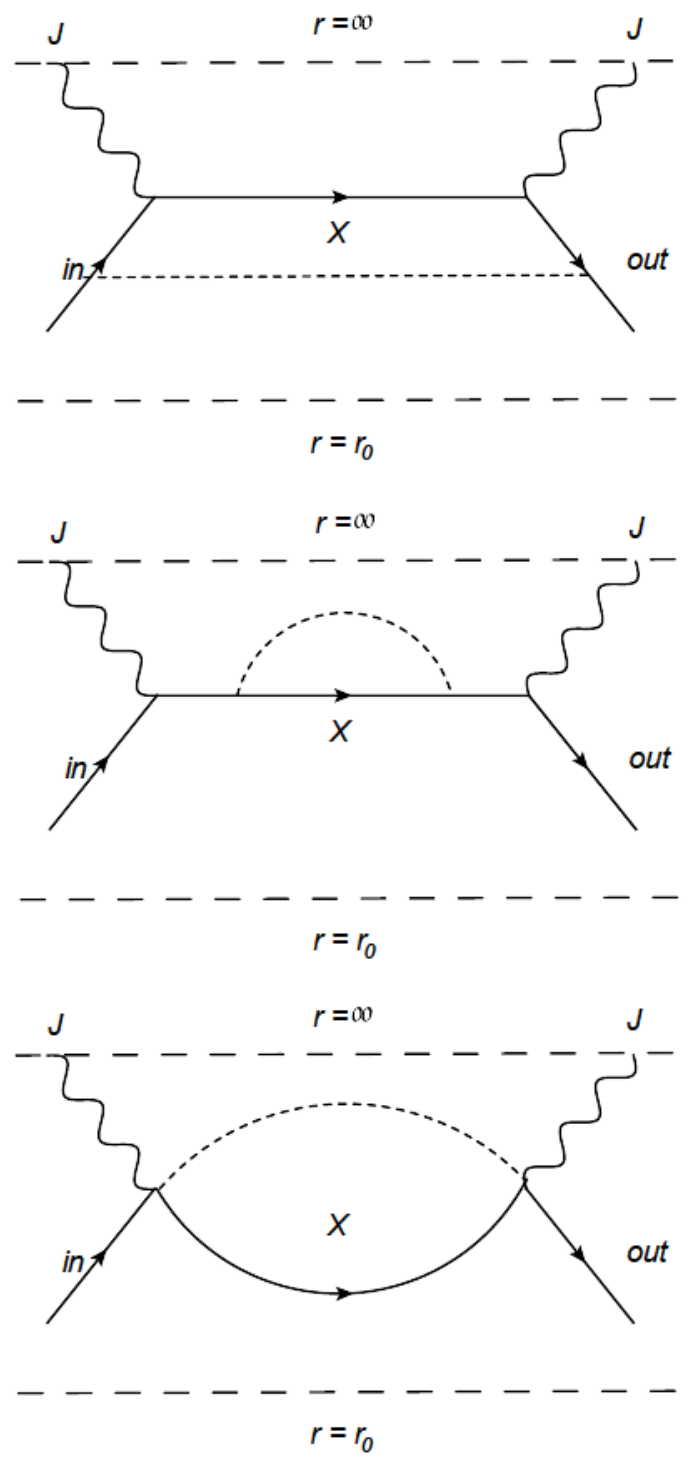

Figura 6.4: Ejemplo de algunos de los diagramas de Witten a un loop. Las líneas de trazos representan gravitones $h_{m n}$. Estos diagramas son correcciones no dominantes a los diagramas de Feynman de la dispersión Compton que contribuyen al DIS en cuatro dimensiones. Estos diagramas están compuestos por campos de cinco dimensiones obtenidos de la reducción dimensional sobre una variedad de Einstein compacta. La contribución de estos diagramas es de orden $N^{-2}$ relativa al orden dominante de la expansión $1 / N$ que se muestra en la Figura 6.3 . 
puede escribirse

$$
S_{\text {interaction }}^{S F}=i \mathcal{Q} \mu_{p}\left(\pi \alpha^{\prime}\right)^{2} R^{p-4} \int d^{5} x \sqrt{-\operatorname{det} g^{5}}\left(\frac{\rho}{R}\right)^{(\alpha+\beta)+(p-4)\left(1+\frac{\beta}{2}\right)} A^{m}\left[b_{n}^{*} f^{m n}-b_{n} f^{m n *}\right] .
$$

Si consideramos una fluctuación arbitraria en la métrica, aparecen los términos de interacción

$$
h^{m q} F_{q}^{n} F_{m n}^{*} \quad, \quad h^{m q} A_{m}\left(B_{n}^{*} F_{q}^{n}-B_{n}^{*} F_{q}^{n *}\right),
$$

que, tras la reducción dimensional, se convierten en

$$
h^{m q} f_{q}^{n} f_{m n}^{*} \quad ; \quad h^{m q} A_{m}\left(b_{n}^{*} f_{q}^{n}-b_{n}^{*} f_{q}^{n *}\right),
$$

Colectando todos los términos llegamos a la acción completa en cinco dimensiones

$$
\begin{aligned}
S_{\text {total }}^{S F}= & S_{0}^{S F}+S_{\text {interaction }}^{S F} \\
= & -4 \mu_{p}\left(\pi \alpha^{\prime}\right)^{2} R^{p-4} \int d^{5} x \sqrt{-\operatorname{det} g^{5}}\left(\frac{\rho}{R}\right)^{(\alpha+\beta)+(p-4)\left(1+\frac{\beta}{2}\right)}\left[\frac{1}{4} f^{m n} f_{m n}^{*}+\frac{1}{2} M_{\ell}^{2} b^{m} b_{m}^{*}+\right. \\
& \left.\frac{1}{4} h^{m q} f_{q}^{n} f_{m n}^{*}-\frac{i}{4} \mathcal{Q} A^{m}\left(b_{n}^{*} f^{m n}-b_{n} f^{m n *}\right)-\frac{i}{4} \mathcal{Q} h^{m q} A_{m}\left(b_{n}^{*} f_{q}^{n}-b_{n}^{*} f_{q}^{n *}\right)\right] .
\end{aligned}
$$

Podemos redefinir los campos para que los términos cinéticos queden canónicamente normalizados en términos de potencias de $N$,

$$
\begin{aligned}
\widetilde{b}^{m} & \equiv \sqrt{N} b^{m}, \quad\left(\widetilde{f}^{m n} \equiv \sqrt{N} f^{m n}\right) \\
\widetilde{A}^{m} & \equiv N A^{m}, \\
\widetilde{h}^{m n} & \equiv N h^{m n} .
\end{aligned}
$$

Utilizando el factor 6.6.17 podemos escribir la acción $S_{\text {total }}^{S F}$ exclusivamente en términos de $N$ como

$$
\begin{aligned}
S_{\text {total }}^{S F}= & \frac{R^{p-4}}{2^{p-2} \pi^{p-2} \lambda \alpha^{\prime \frac{p-3}{2}}} \int d^{5} x \sqrt{-\operatorname{det} g^{5}}\left(\frac{\rho}{R}\right)^{(\alpha+\beta)+(p-4)\left(1+\frac{\beta}{2}\right)} \\
& {\left[\frac{1}{4} \widetilde{f}^{m n} \widetilde{f}_{m n}^{*}+\frac{1}{2} M_{\ell}^{2} \widetilde{b}^{m} \widetilde{b}_{m}^{*}+N^{-1} \frac{1}{4} \widetilde{h}^{m q} \widetilde{f}_{q}^{n} \widetilde{f}_{m n}^{*}-\right.} \\
& \left.N^{-1} \frac{i}{4} \mathcal{Q} \widetilde{A}^{m}\left(\widetilde{b}_{n}^{*} \widetilde{f}^{m n}-\widetilde{b}_{n} f^{m n *}\right)-N^{-2} \frac{i}{4} \widetilde{\mathcal{Q}}^{m q} \widetilde{A}_{m}\left(\widetilde{b}_{n}^{*} \widetilde{f}_{q}^{n}-\widetilde{b}_{n}^{*} \widetilde{f}_{q}^{n *}\right)\right]
\end{aligned}
$$

Los diagramas relevantes para este proceso son muy similares a los encontrados en le caso escalar y graficados en las Figuras 6.3 y 6.4, simplemente teniendo en cuenta que las líneas contínuas representan ahora el mesón $b^{m}$ en lugar del mesón escalar $\varphi$ del caso anterior. Observamos nuevamente que solamente el diagrama tipo árbol de la Figura 6.3 contribuye al orden dominante $N^{-2}$, mientras que los de la figura 6.4 son de orden $N^{-4}$ y están suprimidos.

\section{Correcciones $\frac{1}{N}$ para mesones vectoriales con $N_{f}>1$}

El término relevante del lagrangiano para los mesones vectoriales con $N_{f}>1$, de 6.5 .2 , es

$$
\mathcal{L}_{0}^{M F}=-\mu_{p}\left(\pi \alpha^{\prime}\right)^{2} \sqrt{-\operatorname{det} g} \operatorname{Tr}\left\{\widehat{F}_{a b} \widehat{F}^{a b *}+\left(i \widehat{F}_{a b}^{*}\left[B^{a}, B^{b}\right]+c . c .\right)-\left[B_{a}^{*}, B_{b}^{*}\right]\left[B^{a}, B^{b}\right]\right\}
$$


con $\widehat{F}_{a b}=\partial_{a} B_{b}-\partial_{b} B_{a}=F_{a b}-i\left[B_{a}, B_{b}\right]$. Definimos

$$
\begin{gathered}
B^{a}\left(\rho, y^{\mu}, \Omega\right)=\sum_{\ell} b^{a \ell}\left(\rho, y^{\mu}\right) Y^{\ell}(\Omega), \\
\hat{f}_{m n}^{\ell}=\partial_{m} b_{n}^{\ell}-\partial_{n} b_{m}^{\ell}=f_{a b}^{\ell}-i\left[b_{a}^{\ell}, b_{b}^{\ell}\right] .
\end{gathered}
$$

Con esto, escribimos la acción libre reducida a cinco dimensiones com45

$$
\begin{aligned}
S_{0}^{M F}= & -2 \mu_{p}\left(\pi \alpha^{\prime}\right)^{2} R^{p-4} \int d^{5} x \sqrt{-\operatorname{det} g^{5}}\left(\frac{\rho}{R}\right)^{(\alpha+\beta)+(p-4)\left(1+\frac{\beta}{2}\right)} \operatorname{Tr}\left\{\frac{1}{2} \widehat{f}^{m n \ell} \widehat{f}_{m n}^{\ell *}+M_{\ell}^{2} b^{m \ell} b_{m}^{\ell *}+\right. \\
& \left.\sum_{\ell^{\prime} \ell^{\prime \prime}}\left[\frac{i}{2} a_{\ell \ell^{\prime} \ell^{\prime \prime}}\left[b_{m}^{\ell^{\prime}}, b_{n}^{\ell^{\prime \prime}}\right] \widehat{f}^{m n \ell *}+c . c .\right]-\sum_{\ell^{\prime} \ell^{\prime \prime} \ell^{\prime \prime \prime}} \frac{c_{\ell \ell^{\prime} \ell^{\prime \prime} \ell^{\prime \prime \prime}}}{2}\left[b_{m}^{\ell}, b_{n}^{\ell^{\prime}}\right]\left[b^{m \ell^{\prime \prime} *}, b^{n \ell^{\prime \prime \prime} *}\right]\right\}, \quad \text { (6.6.28) }
\end{aligned}
$$

donde hemos usado 6.6.9) y 6.6.10), así como definido

$$
\begin{aligned}
a_{\ell \ell^{\prime} \ell^{\prime \prime}} & \equiv \int d^{p-4} \Omega \sqrt{\widetilde{g}} Y^{\ell *} Y^{\ell^{\prime}} Y^{\ell^{\prime \prime}}, \\
c_{\ell \ell^{\prime} \ell^{\prime \prime} \ell^{\prime \prime \prime}} & \equiv \int d^{p-4} \Omega \sqrt{\widetilde{g}} Y^{\ell} Y^{\ell^{\prime}} Y^{\ell^{\prime \prime} *} Y^{\ell^{\prime \prime \prime} *} .
\end{aligned}
$$

Luego de la reducción a cinco dimensiones, el lagrangiano 6.5.8

$$
\begin{aligned}
\mathcal{L}_{\text {interaction }}^{M F}= & i \mathcal{Q} \mu_{p}\left(\pi \alpha^{\prime}\right)^{2} \sqrt{-\operatorname{det} g}\left\{A_{m} \operatorname{Tr}\left(B_{n}^{*} \widehat{F}^{n m}-B_{n}\left(\widehat{F}^{n m}\right)^{*}\right)+\right. \\
& \left.i A_{m} \operatorname{Tr}\left(B_{n}^{*}\left[B^{m}, B^{n}\right]\right)+i A_{m} \operatorname{Tr}\left(B_{n}\left[B^{m *}, B^{n *}\right]\right)\right\} \\
\equiv & \mathcal{L}_{\text {interation }_{1}}^{M F}+\mathcal{L}_{\text {interation }_{2}}^{M F}+\mathcal{L}_{\text {interation }_{3} .}^{M F}
\end{aligned}
$$

puede escribirse

$$
\begin{aligned}
S_{\text {interaction }}^{M F}= & \mathcal{Q} \mu_{p}\left(\pi \alpha^{\prime}\right)^{2} \int d^{5} x \sqrt{-\operatorname{det} g^{5}}\left(\frac{\rho}{R}\right)^{(\alpha+\beta)+(p-4)\left(1+\frac{\beta}{2}\right)} \\
& \left\{i A^{m} \operatorname{Tr}\left(b_{n}^{\ell *} \widehat{f}^{m n \ell}-b_{n}^{\ell} \widehat{f}^{m n \ell *}\right)-\sum_{\ell^{\prime} \ell^{\prime \prime}}\left(a_{\ell \ell^{\prime} \ell^{\prime \prime}} A_{m} \operatorname{Tr}\left(b_{n}^{\ell *}\left[b^{m \ell^{\prime}}, b^{n \ell^{\prime \prime}}\right]\right)+\text { c.c. }\right)\right\} .
\end{aligned}
$$

Las perturbaciones de gravitones introducen los términos de interacción

$$
h^{m q} \operatorname{Tr}\left(\widehat{F}_{q}^{n} \widehat{F}_{m n}^{*}\right) \quad ; \quad h^{m q} \operatorname{Tr}\left[A_{m}\left(B_{n}^{*} \widehat{F}_{q}^{n}-B_{n}^{*} \widehat{F}_{q}^{n *}\right)\right],
$$

que, luego de la reducción dimensional, quedan

$$
h^{m q} \operatorname{Tr}\left(\widehat{f}_{q}^{n} \widehat{f}_{m n}^{*}\right) \quad ; \quad h^{m q} \operatorname{Tr}\left[A_{m}\left(b_{n}^{*} \widehat{f}_{q}^{n}-b_{n}^{*} \widehat{f}_{q}^{n *}\right)\right] .
$$

\footnotetext{
${ }^{45}$ Esta es la parte de la acción correspondiente a un solo $b^{\ell}$.
} 
Uniendo todos los factores, queda

$$
\begin{aligned}
S_{\text {total }}^{M F}= & S_{0}^{M F}+S_{\text {interaction }}^{M F} \\
= & -2 \mu_{p}\left(\pi \alpha^{\prime}\right)^{2} R^{p-4} \int d^{5} x \sqrt{-\operatorname{det} g^{5}}\left(\frac{\rho}{R}\right)^{(\alpha+\beta)+(p-4)\left(1+\frac{\beta}{2}\right)} \\
& \operatorname{Tr}\left\{\frac{1}{2} \widehat{f}^{m n \ell} \widehat{f}_{m n}^{\ell *}+M_{\ell}^{2} b^{m \ell} b_{m}^{\ell *}+\sum_{\ell^{\prime} \ell^{\prime \prime}}\left(\frac{i}{2} a_{\ell \ell^{\prime} \ell^{\prime \prime}}\left[b_{m}^{\ell^{\prime}}, b_{n}^{\ell^{\prime \prime}}\right] \widehat{f}^{m n \ell *}+c . c .\right)\right. \\
& \left.-\sum_{\ell^{\prime} \ell^{\prime \prime} \ell^{\prime \prime \prime}} \frac{c_{\ell \ell^{\prime} \ell^{\prime \prime} \ell^{\prime \prime \prime}}}{2}\left[b_{m}^{\ell}, b_{n}^{\ell^{\prime}}\right]\left[b^{m \ell^{\prime \prime *} *}, b^{n \ell^{\prime \prime \prime} *}\right]\right\}+\frac{1}{2} h^{m q} \operatorname{Tr}\left(\widehat{f}_{q}^{n \ell} \widehat{f}_{m n}^{\ell *}\right) \\
& -\frac{i}{2} \mathcal{Q} A^{m} \operatorname{Tr}\left(b_{n \ell}^{*} \widehat{f}^{m n \ell}-b_{n}^{\ell} \widehat{f}^{m n \ell *}\right)-\frac{i}{2} \mathcal{Q} h^{m q} A_{m} \operatorname{Tr}\left(b_{n}^{\ell *} \widehat{f}_{q}^{n \ell}-b_{n}^{\ell *} \widehat{f}_{q}^{n \ell *}\right)+ \\
& {\left.\left[\frac{i}{2} \sum_{\ell^{\prime} \ell^{\prime \prime}} a_{\ell \ell^{\prime} \ell^{\prime \prime}} A_{m} \operatorname{Tr}\left(b_{n \ell}^{*}\left[b^{m \ell^{\prime}}, b^{n \ell^{\prime \prime}}\right]\right)+c . c .\right]\right\} . }
\end{aligned}
$$

$\mathrm{Al}$ igual que en los casos anteriores, redefinimos los campos para que queden canónicamente normalizados

$$
\begin{aligned}
\widetilde{b}^{m} & \equiv \sqrt{N} b^{m}, \quad\left(\widetilde{\widetilde{f}}^{m n} \equiv \sqrt{N} \widehat{f}^{m n}\right) \\
\widetilde{A}^{m} & \equiv N A^{m}, \\
\widetilde{h}^{m n} & \equiv N h^{m n} .
\end{aligned}
$$

Usando el desarrollo de 6.6.17), escribimos la $S_{\text {total }}^{M F}$ en términos de potencias de $N$ como

$$
\begin{aligned}
& S_{\text {total }}^{M F}=\frac{R^{p-4}}{2^{p-1} \pi^{p-2} \lambda \alpha^{\prime \frac{p-3}{2}}} \int d^{5} x \sqrt{-\operatorname{det} g^{5}}\left(\frac{\rho}{R}\right)^{(\alpha+\beta)+(p-4)\left(1+\frac{\beta}{2}\right)} \\
& \operatorname{Tr}\left\{\frac{1}{2} \widetilde{\widetilde{f}}^{-m n \ell \widetilde{\widetilde{f}}_{m n}^{\ell *}}+M_{\ell}^{2} \widetilde{b}^{m \ell \ell} \widetilde{b}_{m}^{\ell *}+N^{-\frac{1}{2}} \sum_{\ell^{\prime} \ell^{\prime \prime}}\left(\frac{i}{2} a_{\ell \ell^{\prime} \ell^{\prime \prime}}\left[\widetilde{b}_{m}^{\ell^{\prime}}, \widetilde{b}_{n}^{\ell^{\prime \prime}}\right] \widetilde{\widetilde{f}}^{m n \ell *}+c . c .\right)\right. \\
& \left.\left.-N^{-1} \sum_{\ell^{\prime} \ell^{\prime \prime} \ell^{\prime \prime \prime}} \frac{c_{\ell \ell^{\prime} \ell^{\prime \prime} \ell^{\prime \prime \prime}}}{2}\left[\widetilde{b}_{m}^{\ell}, \widetilde{b}_{n}^{\ell^{\prime}}\right] \widetilde{b}^{m \ell^{\prime \prime} *}, \widetilde{b}^{n \ell^{\prime \prime \prime} *}\right]\right\}+N^{-1} \frac{1}{2} \widetilde{h}^{m q} \operatorname{Tr}\left(\widetilde{\widetilde{f}}_{q} \frac{\sim \widetilde{f_{m}}}{m n}\right) \\
& -N^{-1} \frac{i}{2} \mathcal{Q} A^{m} \operatorname{Tr}\left(\widetilde{b}_{n \ell}^{*} \widetilde{\widetilde{f}}^{m n \ell}-\widetilde{b}_{n}^{\ell} \widetilde{\widetilde{f}}^{m n \ell *}\right)-N^{-2} \frac{i}{2} \mathcal{Q} \widetilde{h}^{m q} A_{m} \operatorname{Tr}\left(\widetilde{b}_{n}^{\ell *} \widetilde{\widetilde{f}}_{q}^{n \ell}-\widetilde{b}_{n}^{\ell *} \widetilde{\widetilde{f}}_{q}^{n \ell *}\right)+ \\
& \left.\left.N^{-\frac{3}{2}}\left[\frac{i}{2} \sum_{\ell^{\prime} \ell^{\prime \prime}} a_{\ell \ell^{\prime} \ell^{\prime \prime}} A_{m} \operatorname{Tr}\left(\widetilde{b}_{n \ell}^{*} \widetilde{b}^{m \ell^{\prime}}, \widetilde{b}^{n \ell^{\prime \prime}}\right]\right)+\text { c.c. }\right]\right\} \text {. }
\end{aligned}
$$

Dado que en este caso tenemos más vértices que en el anterior, podemos construir más diagramas relevantes para nuestro proceso de interés. Estos son, además de los de las Figuras 6.3 y 6.4 los que aparecen en la Figura 6.5. Estos nuevos diagramas son no dominantes en $N$ pero, dado que tienen un loop mesónico, debemos sumar sobre todos los sabores posibles, obteniendo un factor adicional $N_{f}$. Por otro lado, si bien estos tres diagramas son no dominantes respecto del de la Figura 6.3, es de un orden más importante que los de la Figura 6.4, ya que son de orden $\frac{N_{f}}{N}$ respecto al dominante. Recordar que los diagramas de Witten de la Figura 6.4 son de orden $\frac{1}{N^{2}}$ con respecto a los de la Figura 6.3 .

\section{Comentarios sobre las contribuciones de orden mayor en el cálcu- lo de SUGRA}

En esta Sección hemos discutido términos de órdenes no dominantes en la expansión $\frac{1}{N}$ para mesones escalares y vectoriales. Obtuvimos estos términos luego de reescalar los campos mesóni- 

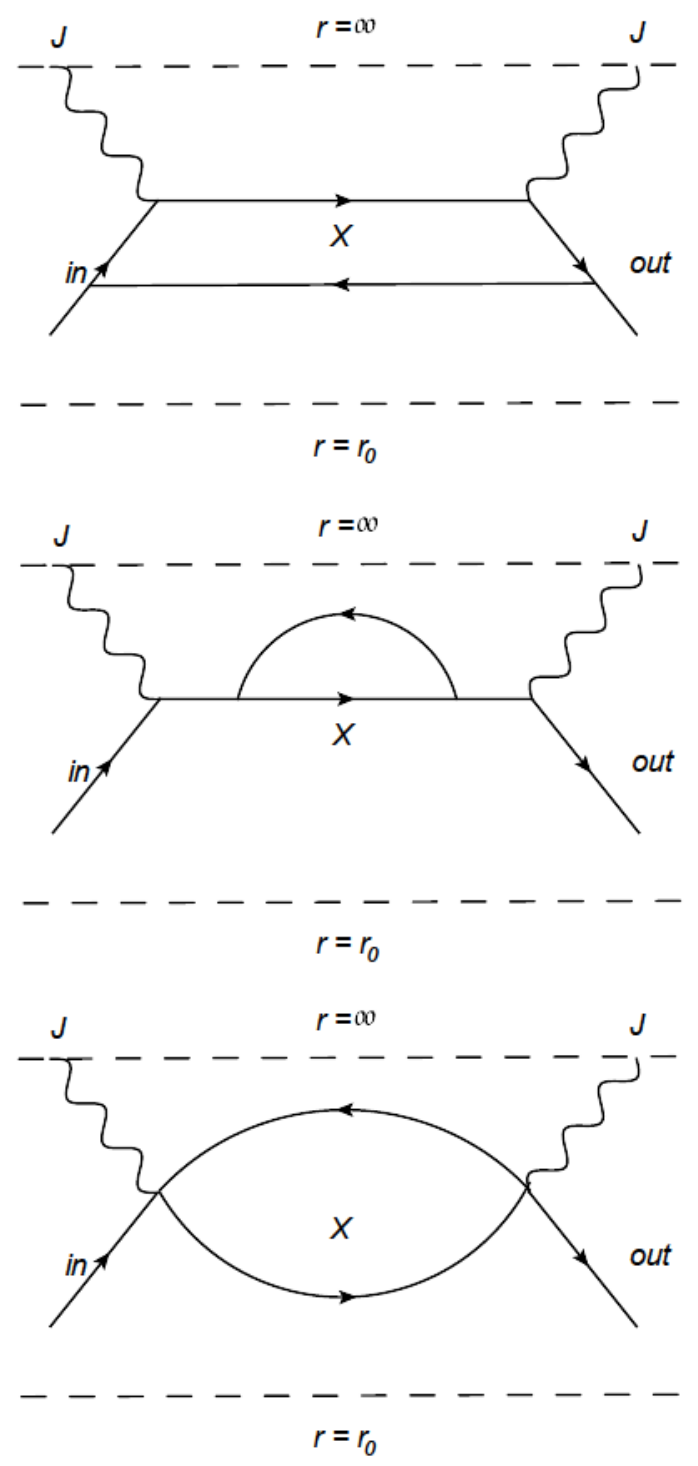

Figura 6.5: Ejemplo de algunos de los diagramas de Witten a un loop. Las líneas contínuas representan el mesón vectorial con $N_{f}>1$. Estos diagramas son correcciones no dominantes a los diagramas de Feynman de la dispersión Compton que contribuyen al DIS en cuatro dimensiones. Estos diagramas están compuestos por campos de cinco dimensiones obtenidos de la reducción dimensional sobre una variedad de Einstein compacta. Estos diagramas contribuyen a orden $\frac{N_{f}}{N}$ respecto del diagrama dominante de la Figura 6.3 . 
cos, resultando esto en una obtención de más vértices que los encontrados en el lagrangiano de primer orden. Con esto construimos más diagramas que son relevantes a la descripción dual holográfica de la dispersión Compton. Los diagramas nuevos se muestran en las Figuras 6.4 6.5 y 6.6 .

En la Figura 6.4 mostramos tres tipos de diagramas de Witten en cinco dimensiones a un loop que incluyen gravitones $h_{m n}$ denotados por la línea de trazos. Estos están compuestos por campos provenientes de la reducción de la acción de supergravedad tipo IIB o IIA según el modelo considerado (D3D7 y D4D6 $\overline{\mathrm{D} 6}$ / D4D8 $\overline{\mathrm{D} 8}$ respectivamente) en una variedad de Einstein de cinco dimensiones. Estos diagramas contribuyen a orden $\frac{1}{N^{2}}$ relativo al diagrama dominante de la Figura 6.3. Notemos que la figura esta no es exhaustiva en cuanto a los diagramas que pueden contribuir a este orden.

En la Figura 6.5 mostramos diagramas de Witten no dominantes que incluyen loops de mesones, denotados por la línea contínua, y solo valen para el caso $N_{f}>1$, donde la autointeracción de los mesones vectoriales está permitida. Debido al loop mesónico, debemos sumar sobre todos los sabores, obteniendo un factor $N_{f}$, con lo que la contribución total de estos diagramas, siempre en referencia al de la Figura 6.3 , será $\frac{N_{f}}{N}$

También podemos considerar contribuciones con múltiples loops como en la Figura 6.6. El diagrama superior de esta figura está compuesto por dos loops mesónicos y contribuirá a orden $\left(\frac{N_{f}}{N}\right)^{2}$, mientras que los otros dos diagramas de esta figura, compuestos por $n$ loops de gravitones, contribuirán a orden $\left(\frac{1}{N^{2}}\right)^{n}$

Resumiendo, las contribuciones estudiadas son

- Figura 6.3 Diagrama dominante.

- Figura 6.4. Contribuciones no dominantes de orden $\frac{1}{N^{2}}$ con respecto a la Figura 6.3 .

- Figura 6.5. Contribuciones no dominantes de orden $\frac{N_{f}}{N}$ con respecto a la Figura 6.3 .

- Figura 6.6. Contribuciones no dominantes de orden $\left(\frac{N_{f}}{N}\right)^{2}$ (diagrama superior) y $\left(\frac{1}{N^{2}}\right)^{n}$ (diagramas siguientes) con respecto a la Figura 6.3.

No hemos obtenido explícitamente estas contribuciones subdominantes correspondientes a las Figuras 6.4, 6.5 y 6.6, pues el completamiento UV de estos diagramas deberá ser llevado a cabo en términos de la teoría de cuerdas y no con el formalismo de SUGRA aquí utilizado. Sin embargo, dichas contribuciones podrían calcularse de manera efectiva en el contexto de supergravedad imponiendo un cutoff UV.

\section{Comentarios sobre la expansión OPE en la teoría de campos}

En esta Subsección relacionamos las expansiones en $\frac{1}{N}$ y $\frac{N_{f}}{N}$ discutidas en la última Subsección a partir de expansiones en supergravedad con las expansiones correspondientes del OPE de dos corrientes en las teorías de gauge de cuatro dimensiones. Partimos del tensor $T_{\mu \nu}$, cuyo valor de expectación entra en la definición del tensor hadrónico $W_{\mu \nu}$. Este está dado por el producto de dos corrientes

$$
\hat{T}_{\mu \nu} \equiv i \int d^{4} x e^{i q \cdot x} \hat{T}\left(\hat{J}_{\mu}(z) \hat{J}_{\nu}(0)\right) .
$$

\footnotetext{
${ }^{46}$ Notar que se incluye el símbolo de operador explícitamente. Es deicr, denotamos por ejemplo el operador $T_{\mu \nu}$ como $\hat{T}_{\mu \nu}$.
} 

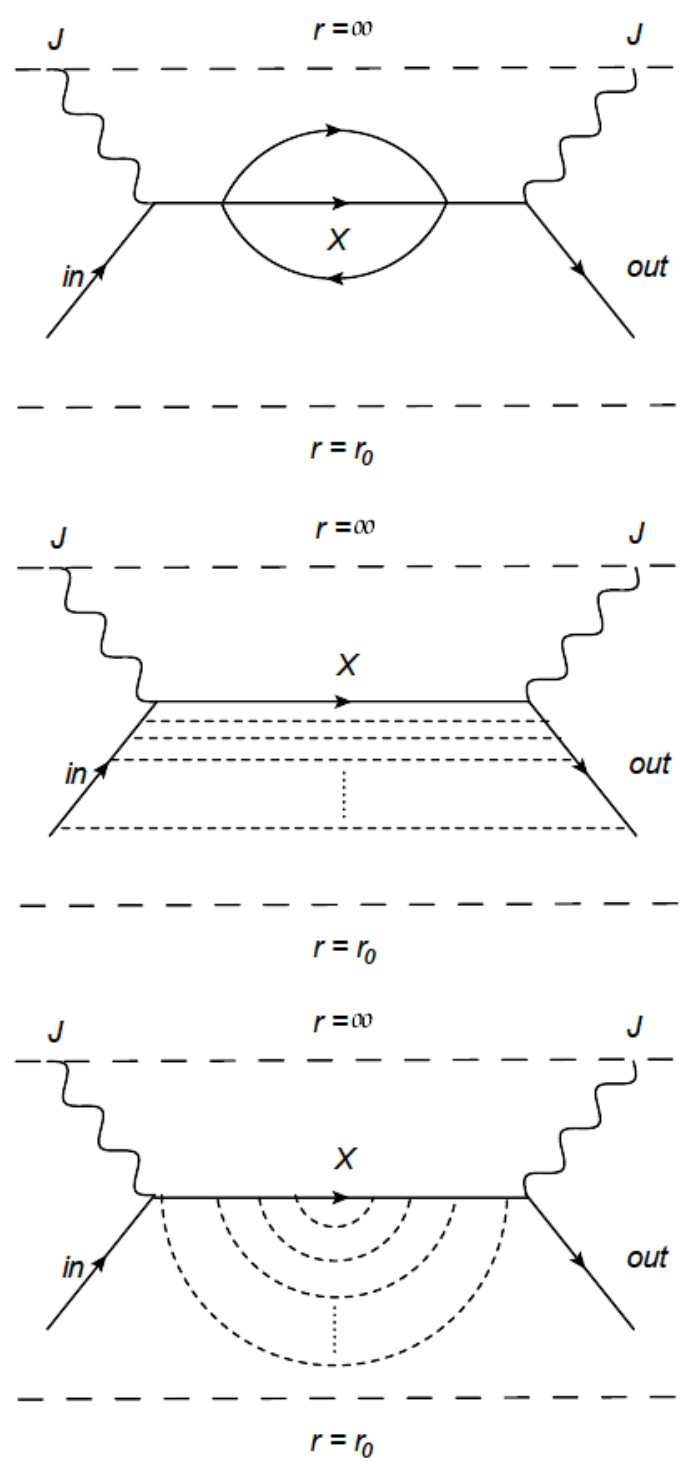

Figura 6.6: Ejemplo de algunos de los diagramas de Witten a más de un loop. Las líneas contínuas representan el mesón vectorial, que en el primer diagrama se refiere exclusivamente al caso $N_{f}>1$. Las líneas de trazos representan los gravitones $h_{m n}$. 
Para el proceso de DIS, los operadores dominantes en el OPE de dos corrientes son de twist 2 para la teoría de gauge débilmente acoplada. A orden cero en QCD podemos escribir ${ }^{47}$

$$
\begin{aligned}
\hat{T}_{\mu \nu} & =\sum_{n=2,4, \cdots}^{\infty} C_{n}^{(1)}\left(-g_{\mu \nu}+\frac{q_{\mu} q_{\nu}}{q^{2}}\right) \frac{2^{n} q_{\mu_{1}} \cdots q_{\mu_{n}}}{\left(-q^{2}\right)^{n}} \hat{O}_{V}^{\mu_{1} \cdots \mu_{n}} \\
& +\sum_{n=2,4, \cdots}^{\infty} C_{n}^{(2)}\left(g_{\mu \mu_{1}}-\frac{q_{\mu} q_{\mu_{1}}}{q^{2}}\right)\left(g_{\nu \mu_{2}}-\frac{q_{\nu} q_{\mu_{2}}}{q^{2}}\right) \frac{2^{n} q_{\mu_{3}} \cdots q_{\mu_{n}}}{\left(-q^{2}\right)^{n-1}} \hat{O}_{V}^{\mu_{1} \cdots \mu_{n}} \\
& +\sum_{n=1,3, \cdots}^{\infty} C_{n}^{(3)} i \epsilon_{\mu \nu \lambda \mu_{1}} q^{\lambda} \frac{2^{n} q_{\mu_{2}} \cdots q_{\mu_{n}}}{\left(-q^{2}\right)^{n}} \hat{O}_{A}^{\mu_{1} \cdots \mu_{n}}
\end{aligned}
$$

donde los coeficientes de Witten son $C_{n}^{(1)}=C_{n}^{(2)}=C_{n}^{(3)}=1+\mathcal{O}\left(\alpha_{s}\right)$, con $\alpha_{s}=\frac{g_{Y M}^{2}}{4 \pi}$ el acoplamiento de QCD en unidades naturales. Los operadores se definen como

$$
\begin{aligned}
& \hat{O}_{V}^{\mu_{1} \cdots \mu_{n}}=\frac{1}{2}\left(\frac{i}{2}\right)^{n-1} \hat{S}\left(\bar{\psi} \gamma^{\mu} \hat{D}^{\mu_{1}} \cdots \hat{D}^{\mu_{n}} \hat{\mathcal{Q}}_{q c m}^{2} \psi\right), \\
& \hat{O}_{A}^{\mu_{1} \cdots \mu_{n}}=\frac{1}{2}\left(\frac{i}{2}\right)^{n-1} \hat{S}\left(\bar{\psi} \gamma^{\mu} \hat{D}^{\mu_{1}} \cdots \hat{D}^{\mu_{n}} \gamma_{5} \hat{\mathcal{Q}}_{q c m}^{2} \psi\right),
\end{aligned}
$$

donde el operador derivada $\hat{D}^{\mu}$ actúa a derecha e izquierda, mientras que $\hat{\mathcal{Q}}_{q c m}$ es la matriz de cargas de los quarks $\psi$ y $\hat{S}$ indica una simetrización y remueve las trazas sobre $\mu_{1} \cdots \mu_{n}$.

Para calcular las funciones de estructura en este régimen hace falta calcular el elemento de matriz de $T_{\mu \nu}$ entre dos estados hadrónicos en el presente caso de espín 1, lo que lleva a

$$
\begin{aligned}
& <P, E\left|\hat{O}_{V}^{\mu_{1} \cdots \mu_{n}}\right| P, E>=\hat{S}\left[a_{n} P^{\mu_{1}} \cdots P^{\mu_{n}}+d_{n}\left(E^{* \mu_{1}} E^{\mu_{2}}-\frac{1}{3} P^{\mu_{1}} P^{\mu_{2}}\right) P^{\mu_{3}} \cdots P^{\mu_{n}}\right] \\
& <P, E\left|\hat{O}_{A}^{\mu_{1} \cdots \mu_{n}}\right| P, E>=\hat{S}\left[r_{n} \epsilon^{\lambda \sigma \tau \mu_{1}} E_{\lambda}^{*} E_{\sigma} P_{r} P^{\mu_{2}} \cdots P^{\mu_{n}}\right]
\end{aligned}
$$

definiendo los coeficientes $a_{n}, d_{n}$ y $r_{n}$.

El diagrama dominante en el modelo de partones es el que se muestra en la Figura 6.7 donde el fotón virtual impacta al partón. Este es un cálculo perturbativo a nivel árbol en la QFT para acoplamiento débil.

Los operadores que aparezcan en el OPE de dos corrientes para acoplamiento débil tienen twist par: $\tau=2,4, \cdots$, y por lo tanto los operadores de trazas simples y twist 2 dominan el OPE. Notemos que para acoplamiento finito estos operadores tienen una dimensión anómala $\gamma_{n}$, donde el subíndice se refiere a los números cuánticos del operador correspondiente. $\mathrm{Al}$ orden dominante en teoría de perturbaciones, $\gamma_{n} \sim \alpha_{s}\left(q^{2}\right) N$ y en este régimen el modelo de partones para espín $\frac{1}{2}$ da la relación de Callan-Gross $F_{2}=2 x F_{1}$, donde la variable de Bjorken $x$ es la fracción del momento total $P^{\mu}$ del hadrón llevado por un dado partón. Decimos que este partón evoluciona, refiriéndonos a que se divide en partones secundarios, lo que hace reducir el momento llevado por cada partón individual.

Por otro lado, para acoplamiento fuerte el comportamiento cambia dramáticamente pues estos operadores tienen dimensión anómala grande y por ende no dominan más la expansión OPE, sino que los que dominarán son operadores de doble traza que no reciben dimensión

\footnotetext{
${ }^{47}$ Esta expresión sigue la notación y métrica de [55], que tiene un signo menos global respecto de lo utilizado en el presente trabajo.
} 


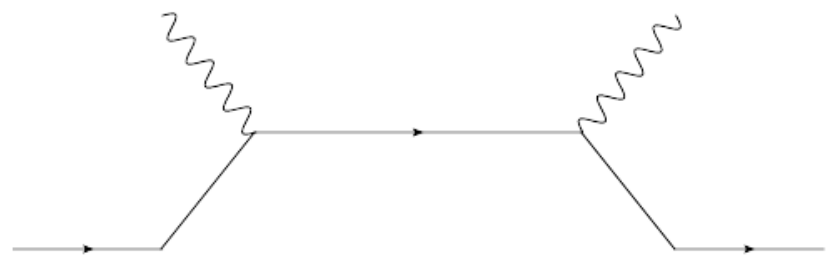

Figura 6.7: Diagrama de quark-antiquark (líneas continuas) representando la dispersión Compton por un mesón en el modelo de partones. Un partón (en este caso un quark) es impactado por el fotón virtual (línea ondulada).

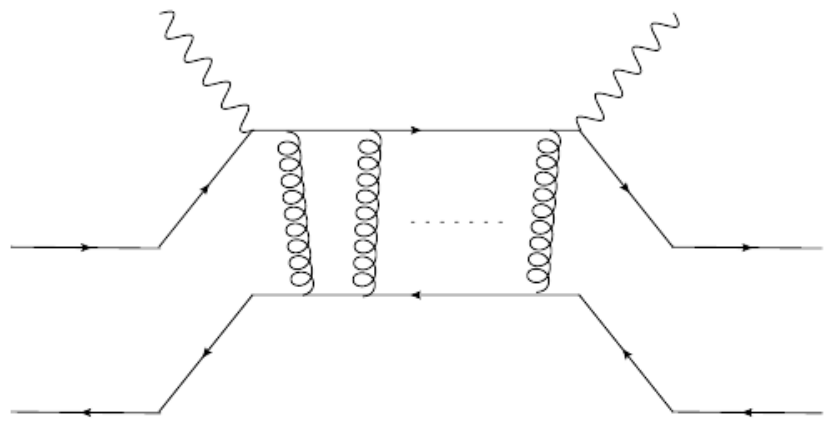

Figura 6.8: Diagrama de quark-antiquark de la dispersión Compton a acoplamiento fuerte. El mesón, esquemáticamente formado por un par quark-antiquark (líneas continuas) es impactado por el fotón virtual (línea ondulada). Este es el diagrama que en el modelo de quarks correspondiente al cálculo dominante del modelo dual de supergravedad de la Figura 6.1. Este es un diagrama planar que representa el intercambio de múltiples gluones entre un par quark-antiquark.

anómala para ningún valor del acoplamiento de 't Hooft $\lambda$. Estos son operadores protegidos. La discusión es análoga al caso de dispersión por glueballs ([5] y Sección 6.1), solo que ahora no solo hay contribuciones de campos en la representación adjunta sino también en la fundamental del grupo de gauge. Para estos, debemos reemplazar el factor $N$ por $\sqrt{N}$ cuando corresponda. Además, tendremos un factor $N_{f}$ proveniente de la suma sobre loops de sabor. La primera diferencia con respecto al caso de acoplamiento débil es que el leptón no puede impactar partones individuales, sino que ahora actúa sobre el hadrón entero. Esto se representa en la Figura 6.8, que representa un intercambio de múltiples gluones en un diagrama planar que puede calcularse en términos del diagrama de Witten de la Figura 6.1 y son los calculados en este Capítulo.

En principio podríamos ir más allá del límite planar, incluyendo diagramas no planares para el intercambio de gluones como se muestra en la Figura 6.9. Este es el diagrama en el modelo de quarks correspondiente a las contribuciones subdominantes del modelo dual de supergravedad dada por el cálculo a un loop de los diagramas mostrados en la Figura 6.4. También pueden considerarse múltiples loops de sabor, como se muestra en la Figura 6.10, que se asocia a los diagramas de la Figura 6.4 . 


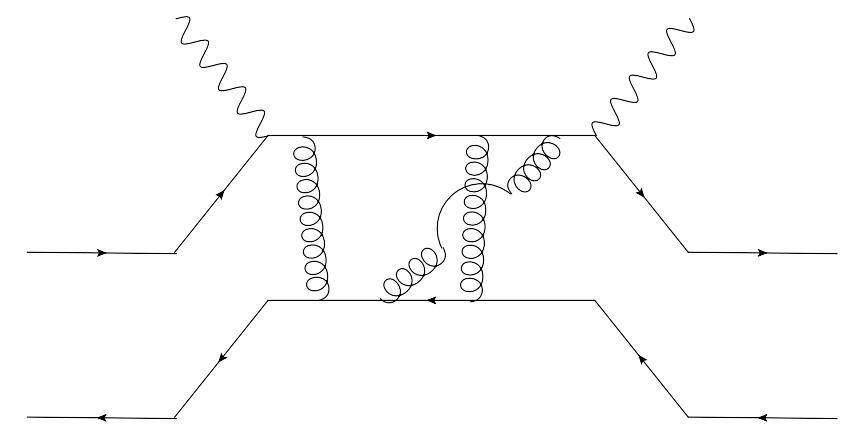

Figura 6.9: Diagrama de quark-antiquark de la dispersión Compton a acoplamiento fuerte. El mesón, esquemáticamente formado por un par quark-antiquark (líneas continuas) es impactado por el fotón virtual (línea ondulada). Este es un diagrama no planar que representa el intercambio de múltiples gluones entre un par quark-antiquark. Las contribuciones de orden $\frac{1}{N^{2}}$ provienen del intercambio no planar de gluones.

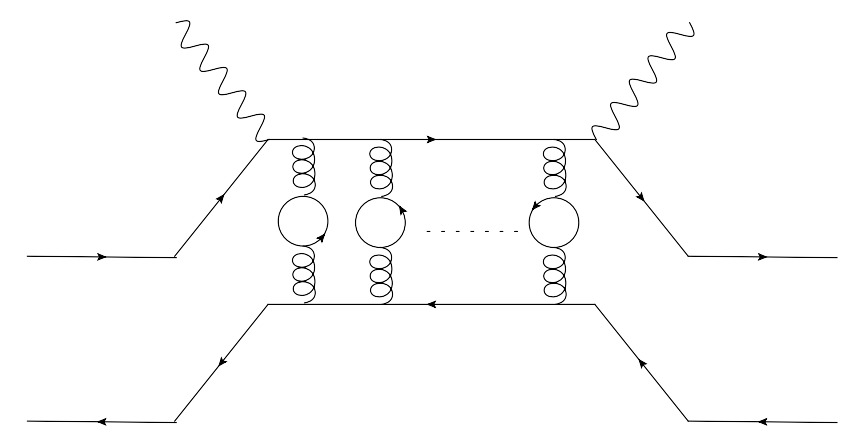

Figura 6.10: Dispersión Compton a acoplamiento fuerte. El mesón (línea continua) es impactado por el fotón virtual (línea ondulada). Este es un diagrama planar que representa el intercambio de múltiples gluones entre un par quark-antiquark, con $n$ loops de sabor. 


\subsection{Discusión de los resultados}

Hemos investigado el DIS por mesones escalares y mesones vectoriales polarizados, obtenidos a partir de diversos modelos holográficos duales con sabores en la representación fundamental del grupo de gauge, que incluyen los modelos de D3D7, D4D8 $\overline{\mathrm{D} 8}$ y $\mathrm{D} 4 \mathrm{D} 6 \overline{\mathrm{D} 6}$-branas. Obtuvimos las funciones de estructura para todo el espectro de mesones escalares y vectoriales, en todos los modelos holográficos estudiados, obteniendo expresiones analíticas para estas funciones en todos los casos. En particular, para los mesones vectoriales obtuvimos la descomposición completa del tensor hadrónico para blancos polarizados de espín 1. Estas son las funciones de estructura $F_{1}, F_{2}, b_{1}, b_{2}, b_{3}, b_{4}, g_{1}$ y $g_{2}$, que fueron calculadas como funciones de $x$ y $t$ para el límite de $|t| \ll 1$. Este problema no había sido considerado en la literatura previa para mesones vectoriales utilizando la correspondencia $A d S / C F T$, por lo que constituye un enfoque totalmente novedoso [1, 2], complementario al desarrollado a partir de expansión en producto de operadores (OPE) en la teoría cuántica de campos estudiado por Hoodbhoy, Jaffe y Manohar [55].

Para los mesones escalares encontramos que $F_{1}=0$, como era de esperarse. Esto se debe a que esta función es proporcional al valor de expectación del operador de Casimir del grupo de Lorentz del hadrón dispersado. Por otro lado $F_{2}$, que da la información independiente del espín, no se anula. La dependencia con el momento intercambiado de esta función es $F_{2} \sim q^{-2\left(\tau_{p}-1\right)}$, lo que corresponde al twist $\tau_{p}=\gamma+2$ en nuestra notación.

Para los mesones vectoriales, puede verse que la dependencia de las funciones de estructura en el parámetro $x$ de Bjorken es similar en todos los modelos. Esto se debe a que estas funciones comparten un factor independiente del modelo, proveniente de la forma de la acción de DBI. Además, poseen otro factor independiente del modelo, que las diferencia ${ }^{48}$

Continuando con el caso de los mesones vectoriales, encontramos la relación de Callan-Gross modificada, ya obtenida para dilatinos en [5] y dada por $2 F_{1}(x)=F_{2}(x)$. Esta es válida para todo valor de $x$ en este rango, siempre en el límite $t \rightarrow 0$. Notemos que las relaciones $2 x F_{1}(x)=F_{2}(x)$ y $2 b_{1}(x)=b_{2}(x)$ corresponden a QCD $(S U(3))$ en el límite perturbativo. En la presente Tesis estudiamos teorías de YM en el límite planar y de acoplamiento fuerte, y en particular en este Capítulo en el régimen $\frac{1}{\sqrt{\lambda}} \ll x \lesssim 1$ (región $\mathbf{A}$ definida en la Introducción). Esto constituye un régimen paramétrico cinemático y dinámico distinto, el cual es inaccesible con pQFT. En el próximo Capítulo estudiaremos los regímenes $e^{-\sqrt{\lambda}} \ll x \ll \frac{1}{\sqrt{\lambda}}$ (región $\mathbf{B}$ ) y $x \sim e^{-\sqrt{\lambda}}$ (región $\mathbf{C}$ ), para los que veremos que las relaciones del tipo Callan-Gross obtenidas adquirirán el factor $x$ usual y explicaremos por qué esto ocurre.

Como está explicado en la citada referencia, la diferencia con la relación de Callan-Gross usual es la falta de un factor $x$ en esta relación, pues esta se escribe $2 x F_{1}(x)=F_{2}(x)$. En el caso de un partón dispersado, que lleva una fracción $x$ del momento total, el OPE da esta relación usual. En el presente caso, en que pensamos el fotón dispersándose por el hadrón completo, la fracción de momento del hadrón dispersado es 1 , lo que se evidencia en la falta de este en la relación de Callan-Gross. También encontramos la relación $2 b_{1}(x)=b_{2}(x)$, a diferencia de la usual $2 x b_{1}(x)=b_{2}(x)$, obtenida también del estudio de los OPE en teoría de campos. Esta última relación proviene del hecho de que $b_{1}$ y $b_{2}$ son obtenidas de la misma torre de operadores que $F_{1}$ y $F_{2}$. También tengamos en cuenta que en el modelo de partones $F_{1}$ representa la probabilidad de encontrar un quark en el hadrón con una fracción de momento $x$, y resulta positiva en nuestros cálculos.

\footnotetext{
${ }^{48}$ Notar que la independencia del modelo de este factor es en cuanto a su estructura formal, dado que este depende del parámetro discreto $\gamma(\ell)$.
} 
También encontramos las nuevas relaciones

$$
\begin{aligned}
b_{2} & =2 b_{1}, \\
b_{1} & =3 F_{1}, \\
g_{2} & =\frac{9}{4 x} F_{1} \\
b_{4} & =-2 b_{3} .
\end{aligned}
$$

que entendemos como predicciones para todos los modelos holográficos estudiados en el límite $t \rightarrow 0$.

La factorización encontrada, en un factor dependiente del modelo y uno independiente, viene de la estructura de la acción de DBI sobre la D $p$-brana de prueba, tanto para el caso abeliano (de un solo sabor, $N_{f}=1$ ) como para su generalización no abeliana (de más de un sabor, $N_{f}>1$ ) [73. Podemos escribir en general el tensor hadrónico $W_{(a)}^{\mu \nu}$ para un dado par de modelos en el límite planar como

$$
W_{(a)}^{\mu \nu}=A_{(a, b)}(x) W_{(b)}^{\mu \nu},
$$

donde $(a)$ y $(b)$ son los modelos y $A_{(a, b)}(x)$ es un factor de conversión que depende de estos. Con esto, podemos escribir las funciones de estructura en la forma $F_{i}^{(a)}(x, t)=A_{(a, b)}(x) F_{i}^{(b)}(x, t)$.

En el límite de $N$ grande encontramos una estructura universal para las funciones de correlación de dos puntos en el acoplamiento fuerte. Para cada modelo holográfico dual encontramos que las funciones de correlación de dos puntos para corrientes de simetría global no abeliana $\left(N_{f}>1\right)$ pueden escribirse como el producto de una constante dependiente del modelo por deltas de Kronecker que indican la conservación de la especie de mesón multiplicando el respectivo resultado abeliano $\left(N_{f}=1\right)$ para el mismo modelo holográfico, ecuación 6.5.19

$$
F_{i}^{(a) M F}(x, t)=C_{f}^{2} \delta_{\mathcal{A B}} F_{i}^{(a) S F}(x, t),
$$

donde $C_{f}$ es el Casimir de $S U\left(N_{f}\right)$ y vale $C_{f}=\frac{1}{2}$ para la representación fundamental. Esta relación nos permite simplificar de manera importante el estudio de los mesones con $N_{f}>1$, reduciéndolo al caso mucho más sencillo de $N_{f}=1$.

Además, hemos investigado las contribuciones de la forma $\frac{1}{N}$ y $\frac{N_{f}}{N}$ al primer orden de cálculo para el tensor hadrónico desde el punto de vista del modelo dual holográfico. En particular, nos concentramos en la estructura de los lagrangianos y los diagramas de Witten relevantes, derivando los primeros. Aunque no hemos calculado los diagramas de Witten explícitamente para las correcciones en $\frac{1}{N}$ y $\frac{N_{f}}{N}$, discutimos cómo surgen de supergravedad, y cómo su dependencia en estos parámetros se corresponde con las expansiones correspondientes en QFT, así como también cómo están suprimidos en potencias de $\frac{1}{N^{2}}$ y $\frac{N_{f}}{N}$ respectivamente en los modelos holográficos duales. 


\section{Capítulo 7}

\section{Estudio del caso $x \ll 1$ : régimen de teoría de cuerdas}

Los cálculos realizados en el Capítulo anterior son válidos siempre que se cumpla la aproximación de supergravedad. Como vemos en la ecuación (6.1.9), para $x \sim \lambda^{-1 / 2}$ la energía asociada a la colisión para un observador local en diez dimensiones se vuelve del orden de la escala de masa. Esto corresponde a la región A definida en la Introducción, $\frac{1}{\sqrt{\lambda}} \ll x<1$. Más allá de este punto, debemos tomar en cuenta el hecho de que la teoría en diez dimensiones es una teoría de supercuerdas. En este Capítulo estudiaremos las regiones B y C definidas en la Introducción, correspondientes a $e^{-\sqrt{\lambda}} \ll x \ll \frac{1}{\sqrt{\lambda}}$ y $x \lesssim e^{-\sqrt{\lambda}}$ respectivamente.

Es importante obtener las funciones de estructura en todo el rango físico del parámetro de Bjorken $x$, y en particular para valores pequeños de este (regiones B, C y D). Del estudio de los momentos para las funciones de estructura $F_{1}$ y $F_{2}[78$ se ve que estas deberían tener un fuerte pico alrededor de $x=0$. Hay un segundo argumento, más físico, para apoyar este comportamiento [79]. En una teoría de gauge débilmente acoplada donde la interacción produjese fragmentación de partones, las funciones de estructura deben aumentar a medida que $x$ disminuye. Si aumentamos la constante de acoplamiento, la evolución de estas se vuelve más rápida. No es claro que esta interpretación sea válida sin más en el presente caso, pues se trata de acoplamiento fuerte y el modelo de partones no es válido. Sin embargo, pensamos que la tendencia debería correr en el mismo sentido. Esto ha sido confirmado por Polchinski y Strassler en [5] para las funciones de estructura de glueballs, como veremos en la Sección 7.1, y por nosotros en [3], como explicaremos en detalle en el presente Capítulo.

En este Capítulo estudiamos tres objetivos principales. El primero es comprobar cómo es el comportamiento de las funciones de estructura en las regiones B y C, correspondientes a $x$ pequeño y exponencialmente pequeño respectivamente. La idea es encontrar esta evolución rápida de las funciones de estructura hacia valores pequeños de $x$ para acoplamiento fuerte para el caso de mesones dinámicos holográficos. El segundo objetivo es mostrar que encontramos una relación de tipo Callan-Gross de la forma $F_{2} \sim 2 x F_{1}$, así como otras similares entre las demás funciones de estructura. Encontraremos un factor extra, independiente de $x$ pero dependiente del modelo, al igual que para el caso de glueballs estudiado en [5]. En tercer lugar mostramos cuán generales son estas nuevas relaciones halladas, es decir, discutimos su comportamiento independiente del modelo. Para calcular las funciones de estructura utilizamos amplitudes de dispersión de cuatro cuerdas cerradas (glueballs, Sección 7.1) y de dos cerradas y dos abiertas (mesones escalares y vectoriales, Secciones 7.2 y 7.3 respectivamente).

En la Sección 7.1 desarrollamos en todo detalle el cálculo para glueballs de [5]. En las Secciones 7.2 y 7.3 estudiamos el caso para el modelo de D3D7-branas para mesones escalares y 
vectoriales respectivamente. En la Sección 7.4 extendemos estos resultados, expresándolos en una forma compacta y general, que incluye los casos de los modelos con D4D6 $\overline{\mathrm{D} 6}$ - y D4D8 $\overline{\mathrm{D} 8}$ branas. En la Sección 7.5 discutiremos el caso de $x$ exponencialmente pequeño (región $\mathbf{C}$ ), y finalizaremos en la Sección 7.6 con una discusión de los resultados.

\subsection{DIS desde glueballs}

En esta Sección estudiaremos el DIS de glueballs para el régimen $x \ll 1$. (regiones B y C). Para esto seguiremos los pasos delineados en [5], aunque lo haremos en mucho mayor detalle que en la citada referencia. Esto nos servirá para presentar el método que utilizaremos en las Secciones posteriores, correspondientes a cálculos originales publicados en [3].

En el Capítulo anterior utilizamos el teorema óptico y sumamos explícitamente sobre los estados intermedios $X$. En el presente caso, donde los cálculos se desarrollan en el marco de la teoría de cuerdas, también utilizaremos el teorema óptico pero calcularemos directamente la amplitud de dispersión de cuatro cuerdas y extraeremos de allí la parte imaginaria. Es claro que no se conoce cómo calcular una amplitud de dispersión en teoría de cuerdas en espacio curvo. Sin embargo, los momentos invariantes son del orden de la escala de cuerdas, por lo que esperaremos que el proceso de dispersión resulte localizado en esta escala. Como esta es pequeña respecto del radio del $A d S$, (pues $\lambda=\frac{R^{4}}{4 \pi \alpha^{\prime 2}} \gg 1 \Rightarrow \alpha^{\prime} \ll R^{2}$ ), haremos el cálculo de la amplitud en espacio plano y luego agregaremos un factor en el elemento de matriz que dé cuenta de la curvatura del $A d S$. Esta localidad será válida para la región $\mathbf{B}$, con $e^{-\sqrt{\lambda}} \ll x \ll \frac{1}{\sqrt{\lambda}}$, pero perderá su validez para la región $\mathbf{C}, x \lesssim e^{-\sqrt{\lambda}}$, que luego consideraremos separadamente. Para poder hacer este pasaje a espacio curvo debemos construir una acción efectiva de supergravedad que reproduzca las amplitudes a nivel árbol de la teoría de supercuerdas. La forma de construir esta acción efectiva, así como la justificación del pasaje de espacio plano a espacio curvo las hemos estudiado en las Secciones 3.1 y 3.2 respectivamente.

El cuadrado de la masa invariante del sistema protón-hadrón en el proceso de DIS es $s \approx \frac{q^{2}}{x}$ para $x$ pequeño, lo que implica que estamos en el régimen de $s$ grande (ver ecuación (6.1.9)). El pomerón [80] es una trayectoria conjeturada de estados de glueball de espín creciente, análogo a la trayectoria del gravitón en teoría de cuerdas. Esta trayectoria da una contribución a las PDF de la forma $f\left(x, q^{2}\right) \sim x^{-\alpha_{0}}$, donde $\alpha_{0}$ es la ordenada al origen de la trayectoria del pomerón en el gráfico $M^{2}$ v.s. $J$ de Regge, $J=\alpha^{\prime} M^{2}+\alpha_{0}$. La variación con $q^{2}$ no ha sido estudiada con argumentos generales pero se cree que debería ser lenta en QCD [81]. Como el proceso con intercambio de pomerón también predice que la sección eficaz total proton-protón debería crecer como $s^{\alpha_{0}}$, la ordenada al origen puede extraerse de los datos $\left(\alpha_{0} \approx 1,1\right)$ o predecirse de QCD $\left(\alpha_{0} \approx 1,4\right)$. Para nuestro estudio alcanza con saber que $1<\alpha_{0}<2$, como fue observado por el experimento HERA.

En $\mathcal{N}=1^{*}$ SYM puede calcularse la ordenada al origen del pomerón para grandes valores de $\lambda$ de manera sencilla. La trayectoria del pomerón es la trayectoria del gravitón en el dual holográfico de $\mathcal{N}=1^{*}$ SYM. Un modo de espín dos, exactamente no masivo daría $\alpha_{0}=2$, mientras que al poner el cutoff en el $A d S_{5} \times W$ aparece una masa (mass gap) de orden $m \sim R^{-2}$, lo que baja la ordenada al origen resultando $\alpha_{0} \sim 2-O\left(\frac{\alpha^{\prime}}{R^{2}}\right) \sim(\lambda)^{-1 / 2}$. Con esto, las funciones de estructura tendrían un comportamiento

$$
x F_{1} \sim F_{2} \propto x^{-1+O\left((\lambda)^{-1 / 2}\right)}
$$

para $x$ pequeño. Veremos que este comportamiento será el obtenido en [5] . 
En toda esta Sección, así como en el resto del Capítulo, consideraremos solamente el comportamiento dominante en la expansión $\frac{1}{N}$, lo que equivale al límite planar de la teoría de gauge. Esto corresponde a la amplitud de dispersión de la teoría de cuerdas a nivel árbol, es decir, vemos la estructura de una sola cuerda, lo que equivale a la dispersión por un solo partón en el modelo holográfico. Como la amplitud de dispersión aumenta con la energía, la producción de múltiples cuerdas, correspondiente al intercambio de múltiples pomerones, domina para $s \rightarrow \infty$ $(x \rightarrow 0)$ para cualquier valor finito de $N$. No estudiaremos este caso, sino el de $N \rightarrow \infty$ estricto.

Para este régimen seguimos factorizando los gravitones como $h^{M N}=(1 / 2)\left(A^{M} v^{N}+A^{N} v^{M}\right)$ donde $A^{m}$ es un campo de gauge $U(1)$ propagándose en el interior del $A d S_{5}$. Para el modelo de Polchinski y Strassler, $v^{i}$ es un vector de Killing constante en la $S^{5}$. En la teoría de supercuerdas estos gravitones, al igual que los dilatones que serán duales a los glueballs en el modelo holográfico, corresponden a cuerdas cerradas. Debemos calcular entonces la amplitud de dispersión de cuatro cuerdas cerradas, $\mathcal{A}_{4}^{4 c, g l u e b a l l}$. Esta amplitud puede expresarse como el producto de un factor cinemático $\mathcal{K}^{4 c, \text { glueball }}$ y un prefactor $\mathcal{P}^{4 c, \text { glueball }}$ que tiene la estructura usual de funciones gamma, y aporta la dependencia en $\alpha^{\prime}$ :

$$
\mathcal{A}_{4}^{4 c, \text { glueball }}=\mathcal{P}^{4 c, \text { glueball }} \mathcal{K}^{4 c, \text { glueball }} .
$$

En la notación de [5], y considerando la relación entre amplitudes de dispersión desde la teoría de cuerdas y lagrangianos efectivos de campos discutido en la Sección 3.1, podemos escribir

$$
S_{\text {int }}^{\text {eff }}=\left.(G K)\right|_{\tilde{t}=0}
$$

donde redefinimos $G \equiv \mathcal{P}^{4 c, \text { glueball }}$, que contendrá la dependencia en $\alpha^{\prime}$, y $K \equiv \mathcal{K}^{4 c, \text { glueball }}$ es un factor cinemático que dará la dependencia en los campos del lagrangiano. $G$ viene dado por [46]

$$
G=-\frac{\alpha^{\prime 3} \tilde{s}^{2}}{64} \prod_{\xi=s, t, u} \frac{\Gamma\left(-\alpha^{\prime} \tilde{\xi} / 4\right)}{\Gamma\left(1+\alpha^{\prime} \tilde{\xi} / 4\right)} .
$$

Las variables $\tilde{s}, \tilde{t}$ y $\tilde{u}$ son las variables de Mandelstam en diez dimensiones planas. Este factor puede reescribirse de manera más sencilla como 1

$$
\left.\operatorname{Im}_{\operatorname{exc}} G\right|_{\tilde{t} \rightarrow 0}=\frac{\pi \alpha^{\prime}}{4} \sum_{m=1}^{\infty} \delta\left(m-\alpha^{\prime} \tilde{s} / 4\right)(m)^{\alpha^{\prime} \tilde{t} / 2}
$$

El último factor, que corresponde al comportamiento de Regge para ángulos pequeños, se volverá importante para las regiones $\mathbf{C}$ y $\mathbf{D}$, de $x$ exponencialmente pequeño.

La idea aquí será utilizar nuevamente la prescripción holográfica 6.1 .35$)$, que iguala la acción de interacción, en este caso proveniente de la teoría de cuerdas, con el elemento de matriz de la teoría de campos de donde extraeremos el tensor hadrónico. Para esto, necesitamos tener un lagrangiano de interacción efectivo desde la teoría de cuerdas, equivalente a $S_{\text {int }}$ en 6.1.27).

Nos interesa el tensor hadrónico, por lo que tenemos que calcular la parte imaginaria de la dispersión Compton. En particular, tenemos que evaluar $K$ en $\tilde{t}=0$, lo que puede hacerse de dos formas a partir de la amplitud de dispersión $\mathcal{A}_{4}^{4 c, \text { glueball }}$.

- (i) La primera es tomar la amplitud $\mathcal{A}_{4}^{4 c, \text { glueball }}$ y reemplazar dos cuerdas cerradas por gravitones $h^{m i}=A^{m} v^{i}$ y las dos restantes por dilatones $\Phi$. Con esto, al tomar el límite $\tilde{t} \rightarrow 0$ obtendremos un lagrangiano de interacción efectivo de cuatro puntos $\mathcal{L}_{h h \Phi \Phi}^{\text {eff }}$ con el que puede calcularse el tensor hadrónico.

\footnotetext{
${ }^{1}$ Los detalles de este cálculo pueden verse en el Apéndice A
} 


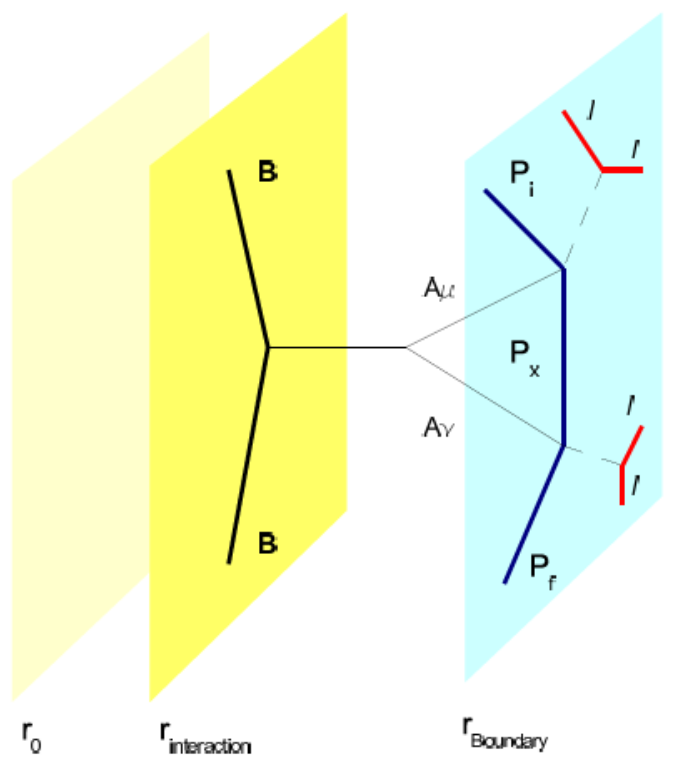

Figura 7.1: Figura esquemática de la descripción holográfica dual del proceso de DIS en la región B: $e^{-\sqrt{\lambda}} \ll x \ll \frac{1}{\sqrt{\lambda}}$. El gravitón que ingresa al interior del $A d S$ representa el intercambio de un pomerón simple en la teoría de gauge dual. Comparar con la Figura 6.1. Figura tomada de nuestro trabajo [3].

- (ii) La segunda opción es partir de la acción de bajas energías de la teoría de cuerdas (supergravedad). En el límite $\alpha^{\prime} \rightarrow 0$ de esta acción, podemos derivar los propagadores para el gravitón y el glueball, así como los vértices de interacción entre estos. Luego calculamos los diagramas correspondientes al proceso de interés: diagrama de contacto, canal $s$, canal $t$ y canal $u$. El coeficiente que acompaña al polo en la amplitud del canal $t$ será el lagrangiano efectivo $\mathcal{L}_{h h \Phi \Phi}^{\text {eff }}$, a menos de un prefactor que contiene la dependencia en $\alpha^{\prime}$. Cuando estudiemos el caso de DIS desde mesones, deberemos considerar no solo $S_{S U G R A}$ sino también $S_{D B I}$ y la acción de interacción entre cuerdas cerradas y abiertas.

Realizaremos el cálculo de ambas maneras para el presente caso, correspondiente a la dispersión de glueballs, comprobando que dan el mismo resultado. Lo mismo sucederá para el caso de mesones escalares, como veremos en la Sección 7.2. Para el caso de mesones vectoriales en la Sección 7.3 asumiremos esta igualdad y solo llevaremos a cabo el cálculo detallado siguiendo la opción (ii). Una representación esquemática de este proceso puede verse en la Figura 7.1.

En la Subsección 7.1.1 realizaremos el cálculo de la amplitud de la manera (i), desde la teoría de cuerdas perturbativa. En la Subsección 7.1.2 repetiremos el cálculo desde la teoría de supergravedad. Calcularemos el lagrangiano efectivo que genera esta misma amplitud en la Subsección 7.1 .3 observando que ambos métodos resultan en nuestro lagrangiano efectivo, 


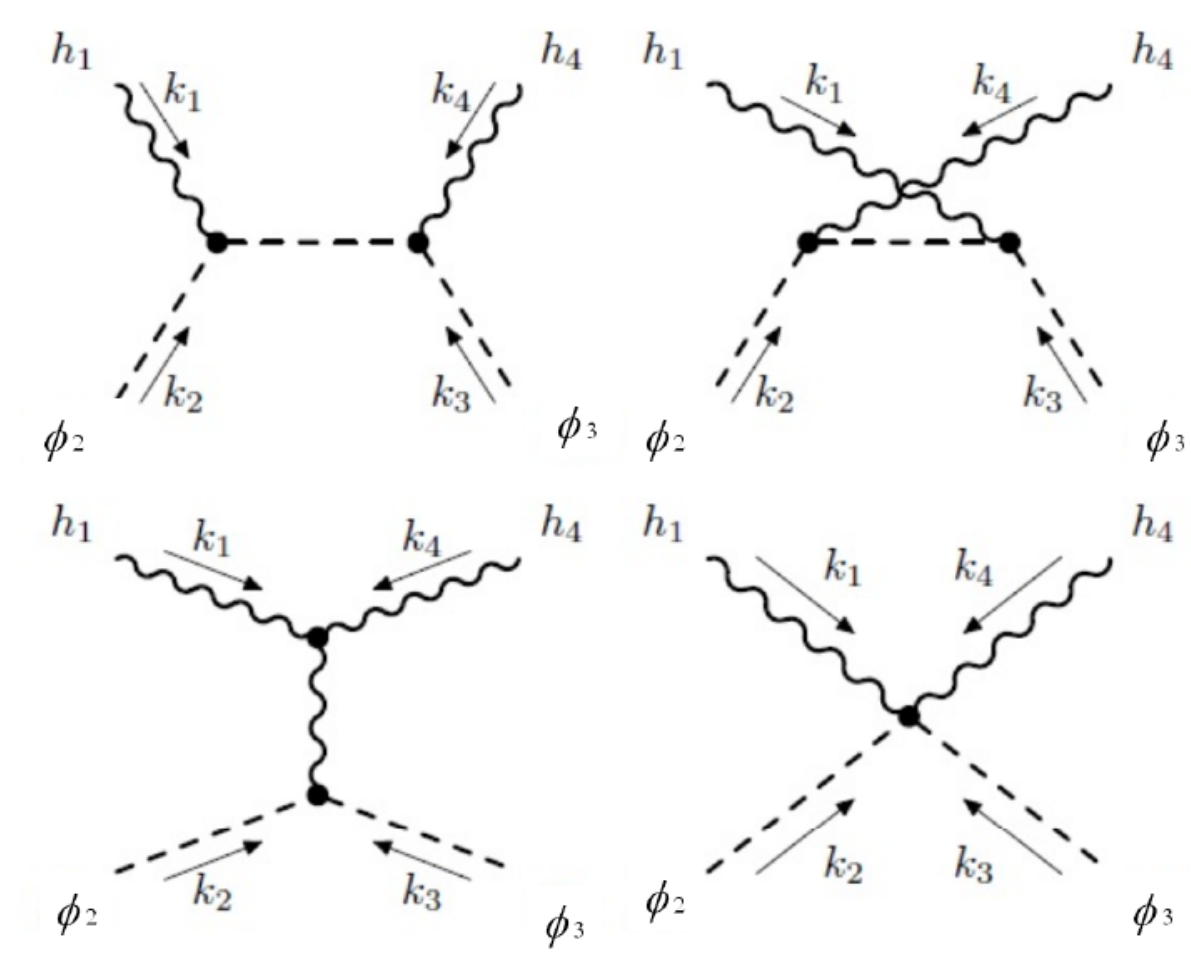

Figura 7.2: Los cuatro diagramas de Feynman correspondientes al cálculo holográfico de la amplitud de dispersión de cuatro puntos: canales $s, u, t$ e interacción de contacto. Las líneas onduladas representan a los gravitones $h_{1}, h_{4}$ así como los intermedios-duales holográficos de los fotones virtuales-, mientras que las líneas de trazos representan a los dilatones $\phi_{2}, \phi_{3} \mathrm{e}$ intermedios -duales holográficos de los glueballs-. Los momentos de los campos se representan con $k_{i}$. Figura tomada de nuestro trabajo [3].

y calcularemos el tensor hadrónico en la Subsección 7.1.4. Finalmente, en la Subsección 7.5 comentaremos el caso de $x$ exponencialmente pequeño (región $\mathbf{C}$ ).

\subsubsection{Amplitud de dispersión a partir de la teoría de cuerdas}

Estamos interesados en la dispersión de dos dilatones $\phi_{2}$ y $\phi_{3}$ y dos gravitones $h_{1}^{\mu \nu}$ y $h_{4}^{\mu \nu}$. Tomamos los cuatro momentos entrantes, como se muestra esquemáticamente en la Figura 7.2

Desde la teoría de cuerdas sabemos que la amplitud de un proceso $h \phi \rightarrow h \phi$ puede escribirse como el producto de dos partes correspondientes a cuerdas abiertas que se propagan hacia la izquierda y hacia la derecha, como vimos en el Capítulo 3 ,

$$
A_{4}^{\text {closed }}=-i \pi^{2} g_{s}^{2} \alpha^{\prime 3} \frac{\Gamma\left(-\frac{\alpha^{\prime} s}{4}\right) \Gamma\left(-\frac{\alpha^{\prime} u}{4}\right) \Gamma\left(-\frac{\alpha^{\prime} t}{4}\right)}{\Gamma\left(1+\frac{\alpha^{\prime} s}{4}\right) \Gamma\left(1+\frac{\alpha^{\prime} u}{4}\right) \Gamma\left(1+\frac{\alpha^{\prime} t}{4}\right)} \times K_{4}^{\text {closed }},
$$

donde $s=-\left(k_{1}+k_{2}\right)^{2}, t=-\left(k_{1}+k_{4}\right)^{2}$ y $u=-\left(k_{1}+k_{3}\right)^{2}$ son las variables de Mandelstam y el prefactor es proporcional a $(s t u)^{-1}$ a primer orden en $\alpha^{\prime}$. El factor cinemático $K_{4}^{\text {closed }}$ contiene a las polarizaciones y no depende de $\alpha^{\prime}$. Puede separarse en dos factores cinemáticos asociados 
con amplitudes de cuerdas abiertas como

$$
K_{4}^{\text {closed }}\left(k_{1}, k_{2}, k_{3}, k_{4}\right) \propto \tilde{K}_{4}^{\text {open }}\left(\frac{1}{2} k_{1}, \frac{1}{2} k_{2}, \frac{1}{2} k_{3}, \frac{1}{2} k_{4}\right) \times K_{4}^{\text {open }}\left(\frac{1}{2} k_{1}, \frac{1}{2} k_{2}, \frac{1}{2} k_{3}, \frac{1}{2} k_{4}\right) .
$$

Cada uno de los factores $K_{4}^{\text {open }}$ puede escribirse en su forma más general como

$$
\begin{aligned}
& K_{4}^{\text {open }}=-\frac{1}{4} \quad\left(s t \rho_{1} \cdot \rho_{3} \rho_{2} \cdot \rho_{4}+s u \rho_{2} \cdot \rho_{3} \rho_{1} \cdot \rho_{4}+t u \rho_{1} \cdot \rho_{2} \rho_{3} \cdot \rho_{4}\right)+ \\
& \frac{1}{2} \quad s\left(\rho_{1} \cdot k_{4} \rho_{3} \cdot k_{2} \rho_{2} \cdot \rho_{4}+\rho_{2} \cdot k_{3} \rho_{4} \cdot k_{1} \rho_{1} \cdot \rho_{3}\right. \\
& \left.+\rho_{1} \cdot k_{3} \rho_{4} \cdot k_{2} \rho_{2} \cdot \rho_{3}+\rho_{2} \cdot k_{4} \rho_{3} \cdot k_{1} \rho_{1} \cdot \rho_{4}\right) \\
& \frac{1}{2} \quad t\left(\rho_{2} \cdot k_{1} \rho_{4} \cdot k_{3} \rho_{3} \cdot \rho_{1}+\rho_{3} \cdot k_{4} \rho_{1} \cdot k_{2} \rho_{2} \cdot \rho_{4}\right. \\
& \left.+\rho_{2} \cdot k_{4} \rho_{1} \cdot k_{3} \rho_{3} \cdot \rho_{4}+\rho_{3} \cdot k_{1} \rho_{4} \cdot k_{2} \rho_{2} \cdot \rho_{1}\right) \\
& \frac{1}{2} \quad u\left(\rho_{1} \cdot k_{2} \rho_{4} \cdot k_{3} \rho_{3} \cdot \rho_{2}+\rho_{3} \cdot k_{4} \rho_{2} \cdot k_{1} \rho_{1} \cdot \rho_{4}\right. \\
& \left.+\rho_{1} \cdot k_{4} \rho_{2} \cdot k_{3} \rho_{3} \cdot \rho_{4}+\rho_{3} \cdot k_{2} \rho_{4} \cdot k_{1} \rho_{1} \cdot \rho_{2}\right)
\end{aligned}
$$

donde $\rho_{i} ; i=1, \ldots, 4$ es la polarización de cada cuerda abierta. Como estamos interesados en el caso $t=0$ debemos poner esta condición en (7.1.8). Dado que las cuerdas son no masivas, $t+s+u=0$, resulta $u=-s$ y con esto

$$
\begin{aligned}
\left.K_{4}^{\text {open }}\right|_{t=0}= & \frac{1}{4} \quad s^{2} \rho_{2} \cdot \rho_{3} \rho_{1} \cdot \rho_{4}+ \\
& \frac{1}{2} \quad s\left[\left(\rho_{1} \cdot k_{4} \rho_{3} \cdot k_{2} \rho_{2} \cdot \rho_{4}+\rho_{2} \cdot k_{3} \rho_{4} \cdot k_{1} \rho_{1} \cdot \rho_{3}\right.\right. \\
& \left.+\rho_{1} \cdot k_{3} \rho_{4} \cdot k_{2} \rho_{2} \cdot \rho_{3}+\rho_{2} \cdot k_{4} \rho_{3} \cdot k_{1} \rho_{1} \cdot \rho_{4}\right)+ \\
& -\left(\rho_{1} \cdot k_{2} \rho_{4} \cdot k_{3} \rho_{3} \cdot \rho_{2}+\rho_{3} \cdot k_{4} \rho_{2} \cdot k_{1} \rho_{1} \cdot \rho_{4}\right. \\
& \left.\left.+\rho_{1} \cdot k_{4} \rho_{2} \cdot k_{3} \rho_{3} \cdot \rho_{4}+\rho_{3} \cdot k_{2} \rho_{4} \cdot k_{1} \rho_{1} \cdot \rho_{2}\right)\right]
\end{aligned}
$$

Ahora tenemos que escribir $K_{\text {left }}^{\text {open }} \otimes K_{\text {right }}^{\text {open }}$ en términos de las polarizaciones $\Theta_{m n}$ de las cuerdas cerradas. Este cálculo resulta

$$
\begin{aligned}
& \left.\frac{1}{s^{2}} \tilde{K}_{4}^{\text {open }} \otimes K_{4}^{\text {open }}\right|_{t=0}=\frac{1}{4}\left[s \tilde{\rho}_{2} \cdot \tilde{\rho}_{3} \tilde{\rho}_{1} \cdot \tilde{\rho}_{4}+\left(\tilde{\rho}_{1} \cdot k_{4} \tilde{\rho}_{3} \cdot k_{2} \tilde{\rho}_{2} \cdot \tilde{\rho}_{4}+\tilde{\rho}_{2} \cdot k_{3} \tilde{\rho}_{4} \cdot k_{1} \tilde{\rho}_{1} \cdot \tilde{\rho}_{3}\right.\right. \\
& \left.+\tilde{\rho}_{1} \cdot k_{3} \tilde{\rho}_{4} \cdot k_{2} \tilde{\rho}_{2} \cdot \tilde{\rho}_{3}+\tilde{\rho}_{2} \cdot k_{4} \tilde{\rho}_{3} \cdot k_{1} \tilde{\rho}_{1} \cdot \tilde{\rho}_{4}\right)+ \\
& -\left(\tilde{\rho}_{1} \cdot k_{2} \tilde{\rho}_{4} \cdot k_{3} \tilde{\rho}_{3} \cdot \tilde{\rho}_{2}+\tilde{\rho}_{3} \cdot k_{4} \tilde{\rho}_{2} \cdot k_{1} \tilde{\rho}_{1} \cdot \tilde{\rho}_{4}\right. \\
& \left.\left.+\quad \tilde{\rho}_{1} \cdot k_{4} \tilde{\rho}_{2} \cdot k_{3} \tilde{\rho}_{3} \cdot \tilde{\rho}_{4}+\tilde{\rho}_{3} \cdot k_{2} \tilde{\rho}_{4} \cdot k_{1} \tilde{\rho}_{1} \cdot \tilde{\rho}_{2}\right)\right] \otimes \\
& {\left[s \rho_{2} \cdot \rho_{3} \rho_{1} \cdot \rho_{4}+\left(\rho_{1} \cdot k_{4} \rho_{3} \cdot k_{2} \rho_{2} \cdot \rho_{4}+\rho_{2} \cdot k_{3} \rho_{4} \cdot k_{1} \rho_{1} \cdot \rho_{3}\right.\right.} \\
& \left.+\rho_{1} \cdot k_{3} \rho_{4} \cdot k_{2} \rho_{2} \cdot \rho_{3}+\rho_{2} \cdot k_{4} \rho_{3} \cdot k_{1} \rho_{1} \cdot \rho_{4}\right)+ \\
& -\left(\rho_{1} \cdot k_{2} \rho_{4} \cdot k_{3} \rho_{3} \cdot \rho_{2}+\rho_{3} \cdot k_{4} \rho_{2} \cdot k_{1} \rho_{1} \cdot \rho_{4}\right. \\
& \left.\left.+\rho_{1} \cdot k_{4} \rho_{2} \cdot k_{3} \rho_{3} \cdot \rho_{4}+\rho_{3} \cdot k_{2} \rho_{4} \cdot k_{1} \rho_{1} \cdot \rho_{2}\right)\right]
\end{aligned}
$$

y extrayendo las polarizaciones de cuerdas $\Theta_{i}^{A A^{\prime}}=\tilde{\rho}_{i}^{A} \otimes \rho_{i}^{A^{\prime}}$ obtenemos

$$
\begin{aligned}
\left.\frac{1}{s^{2}} \tilde{K} \otimes K\right|_{t=0}= & \Theta_{1}^{A A^{\prime}} \Theta_{2}^{B B^{\prime}} \Theta_{3}^{C C^{\prime}} \Theta_{4}^{D D^{\prime}} \times \\
& \left\{s \eta_{A D} \eta_{B C}+\left[k_{A}^{4} k_{C}^{2} \eta_{B D}+k_{B}^{3} k_{D}^{1} \eta_{A C}+k_{A}^{3} k_{D}^{2} \eta_{B C}+k_{B}^{4} k_{C}^{1} \eta_{A D}\right]\right. \\
& \left.-\left[k_{A}^{2} k_{D^{3}}^{3} \eta_{B C}+k_{C}^{4} k_{B}^{1} \eta_{A D}+k_{A}^{4} k_{B}^{3} \eta_{C D}+k_{C}^{2} k_{D}^{1} \eta_{A B}\right]\right\} \times \\
& \left\{s \eta_{A^{\prime} D^{\prime}} \eta_{B^{\prime} C^{\prime}}+\left[k_{A^{\prime}}^{4} k_{C^{\prime}}^{2} \eta_{B^{\prime} D^{\prime}}+k_{B^{\prime}}^{3} k_{D^{\prime}}^{1} \eta_{A^{\prime} C^{\prime}}+k_{A^{\prime}}^{3} k_{D^{\prime}}^{2} \eta_{B^{\prime} C^{\prime}}+k_{B^{\prime}}^{4} k_{C^{\prime}}^{1} \eta_{A^{\prime} D^{\prime}}\right]\right. \\
& \left.-\left[k_{A^{\prime}}^{2} k_{D^{\prime}}^{3} \eta_{B^{\prime} C^{\prime}}+k_{C^{\prime}}^{4} k_{B^{\prime}}^{1} \eta_{A^{\prime} D^{\prime}}+k_{A^{\prime}}^{4} k_{B^{\prime}}^{3} \eta_{C^{\prime} D^{\prime}}+k_{C^{\prime}}^{2} k_{D^{\prime}}^{1} \eta_{A^{\prime} B^{\prime}}\right]\right\}
\end{aligned}
$$

que da un total de 81 términos. 
Las polarizaciones de los gravitones son

$$
\begin{aligned}
\Theta_{1}^{\mu \nu} & =h_{1}^{\mu \nu} \\
\Theta_{4}^{\mu \nu} & =h_{4}^{\mu \nu}
\end{aligned}
$$

donde deben cumplirse las condiciones de transversalidad $k_{1 \mu} h_{1}^{\mu \nu}=k_{4 \mu} h_{4}^{\mu \nu}=0$ y nulidad de la traza $\left(h_{1}\right)_{\mu}^{\mu}=\left(h_{4}\right)_{\mu}^{\mu}=0$. Para los dilatones, la transversalidad viene dada por la forma

$$
\begin{aligned}
\Theta_{2}^{\mu \nu} & =\sqrt{\frac{1}{8}} \phi_{2}\left(\eta^{\mu \nu}-k_{2}^{\mu}{\overline{k_{2}}}^{\nu}-k_{2}^{\nu}{\overline{k_{2}}}^{\mu}\right), \\
\Theta_{3}^{\mu \nu} & =\sqrt{\frac{1}{8}} \phi_{3}\left(\eta^{\mu \nu}-k_{3}^{\mu}{\overline{k_{3}}}^{\nu}-w^{\nu}{\overline{k_{3}}}^{\mu}\right) .
\end{aligned}
$$

donde los momentos auxiliares $\bar{k}_{2}$ y $\bar{k}_{3}$ son tales que $\bar{k}_{2} \cdot \bar{k}_{2}=\bar{k}_{3} \cdot \bar{k}_{3}=0$ y $k_{2} \cdot \bar{k}_{2}=k_{3} \cdot \bar{k}_{3}=1$ y se incluyen únicamente con el fin tener polarizaciones transversales, y si bien resultan importantes en los pasos intermedios del cálculo de esta o de cualquier amplitud, nunca llegarán a formar parte del resultado final. Es decir, se "desacoplan" de cualquier proceso físico.

Se simplifició en gran medida este cálculo con el programa Cadabra [82, 83]. Luego, se reordenó el resultado agrupando los términos en tres categorías distintas, según el tipo de factores que contienen en términos de las polarizaciones de los gravitones: los términos de la primera categoría contienen trazas $\left(h_{1} h_{4}\right) \equiv h_{1 \mu \nu} h_{4}^{\mu \nu}$, los de la segunda contienen un factor del tipo $\left(k_{3} h_{1} h_{4} k_{2}\right) \equiv\left(k_{3 \mu} h_{1}^{\mu \nu} h_{4 \nu \rho} k_{2}^{\rho}\right)$, y en los de la tercera $h_{1}$ y $h_{4}$ no tienen ningún índice contraído entre sí, por lo que se forman factores del tipo $\left(k_{2} h_{1} k_{4}\right)\left(k_{1} h_{4} k_{3}\right) \equiv\left(k_{2 \mu} h_{1}^{\mu \nu} k_{4 \nu}\right)\left(k_{1 \alpha} h_{4}^{\alpha \beta} k_{3 \beta}\right)$. Luego de utilizar las propiedades de transversalidad, conservación de momento $\left(\sum k_{i}=0\right) \mathrm{y}$ luego de escribir los productos de momentos en términos de las variables de Mandelstam

$$
\begin{aligned}
s & =-\left(k_{1}+k_{2}\right)^{2}=-2 k_{1} \cdot k_{2}=-\left(k_{3}+k_{4}\right)^{2}=-2 k_{3} \cdot k_{4}, \\
u & =-\left(k_{1}+k_{3}\right)^{2}=-2 k_{1} \cdot k_{3}=-\left(k_{2}+k_{4}\right)^{2}=-2 k_{2} \cdot k_{4}, \\
t & =-\left(k_{1}+k_{4}\right)^{2}=-2 k_{1} \cdot k_{4}=-\left(k_{2}+k_{3}\right)^{2}=-2 k_{2} \cdot k_{3},
\end{aligned}
$$

y reemplazar $t=-u-s$ obtenemos la expresión final para cada una de las tres categorías.

En los términos que acompañan a la traza $\left(h_{1} h_{4}\right)=h_{1}^{a b} h_{2} a b$ los momentos auxiliares se cancelan automáticamente, y la expresión que obtenemos luego de desacoplar $\bar{k}_{2}$ y $\bar{k}_{3}$ y volver a utilizar la conservación de los momentos es

$$
\left(h_{1} h_{4}\right) s^{2} u^{2}=\left(h_{1} h_{4}\right) s u \frac{1}{2}\left(t^{2}-s^{2}-u^{2}\right) .
$$

Para el segundo conjunto de términos es

$$
\begin{aligned}
& 2 s u\left\{t\left[\left(k_{4} h_{1} h_{4} k_{1}\right)+\left(k_{2} h_{1} h_{4} k_{3}\right)+\left(k_{3} h_{1} h_{4} k_{2}\right)\right)\right. \\
& \left.-u\left[\left(k_{4} h_{1} h_{4} k_{2}\right)+\left(k_{3} h_{1} h_{4} k_{1}\right)\right]-s\left[\left(k_{4} h_{1} h_{4} k_{3}\right)+\left(k_{2} h_{1} h_{4} k_{1}\right)\right]\right\} .
\end{aligned}
$$

Mientras que para la tercera categoría tenemos que la expresión final que multiplica a $\frac{1}{8} h_{1}^{c d} h_{4}^{a b}$ es

$$
4\left[s^{2}\left(k_{3} h_{1} k_{3}\right)\left(k_{2} h_{4} k_{2}\right)+u^{2}\left(k_{2} h_{1} k_{2}\right)\left(k_{3} h_{4} k_{3}\right)+2 s u\left(k_{2} h_{1} k_{3}\right)\left(k_{2} h_{4} k_{3}\right)\right],
$$

En conclusión, sumando las tres contribuciones 7.1.12, 7.1.13 y 7.1.14 podemos decir que el factor cinético de la amplitud del proceso $h \phi \rightarrow h \phi$ calculado en el marco de la teoría supersimétrica de cuerdas tipo IIB resulta ser 


$$
\begin{aligned}
A\left(h_{1}, \phi_{2}, \phi_{3}, h_{4}\right)= & \left(h_{1} h_{4}\right) s u \frac{1}{2}\left(t^{2}-s^{2}-u^{2}\right)+4\left[s^{2}\left(k_{3} h_{1} k_{3}\right)\left(k_{2} h_{4} k_{2}\right)+u^{2}\left(k_{2} h_{1} k_{2}\right)\left(k_{3} h_{4} k_{3}\right)\right. \\
& \left.+2 s u\left(k_{2} h_{1} k_{3}\right)\left(k_{2} h_{4} k_{3}\right)\right]+2 s u\left\{t\left[\left(k_{4} h_{1} h_{4} k_{1}\right)+\left(k_{2} h_{1} h_{4} k_{3}\right)+\left(k_{3} h_{1} h_{4} k_{2}\right)\right]\right. \\
& \left.-u\left[\left(k_{4} h_{1} h_{4} k_{2}\right)+\left(k_{3} h_{1} h_{4} k_{1}\right)\right]-s\left[\left(k_{4} h_{1} h_{4} k_{3}\right)+\left(k_{2} h_{1} h_{4} k_{1}\right)\right]\right\} .
\end{aligned}
$$

Para encontrar el lagrangiano efectivo que buscamos en el régimen en el que, como veremos, el intercambio de un gravitón es el proceso dominante, basta imponer $t=0$ y $u=-s$. Una identidad útil a la hora de rescribir el término asociado a la tercer categoría es la relación

$$
\begin{aligned}
& \left(k_{4} h_{1} k_{3}\right)\left(k_{2} h_{4} k_{1}\right)+\left(k_{4} h_{1} k_{2}\right)\left(k_{3} h_{4} k_{1}\right)-\left(k_{4} h_{1} k_{4}\right)\left(k_{2} h_{4} k_{3}\right)-\left(k_{2} h_{1} k_{3}\right)\left(k_{1} h_{4} k_{1}\right)= \\
& \left(k_{2} h_{1} k_{2}\right)\left(k_{3} h_{4} k_{3}\right)+\left(k_{3} h_{1} k_{3}\right)\left(k_{2} h_{4} k_{2}\right)-2\left(k_{2} h_{1} k_{3}\right)\left(k_{2} h_{4} k_{3}\right),
\end{aligned}
$$

proveniente de la conservación de momentos. El resultado es

$$
\begin{aligned}
& \left.A\left(h_{1}, \phi_{2}, \phi_{3}, h_{4}\right)\right|_{t=0}= \\
& s^{2}\left\{s^{2}\left(h_{1} h_{4}\right)+2 s\left[\left(k_{4} h_{1} h_{4} k_{3}\right)+\left(k_{2} h_{1} h_{4} k_{1}\right)-\left(k_{4} h_{1} h_{4} k_{2}\right)-\left(k_{3} h_{1} h_{4} k_{1}\right)\right]\right. \\
& \left.+4\left[\left(k_{4} h_{1} k_{3}\right)\left(k_{2} h_{4} k_{1}\right)+\left(k_{4} h_{1} k_{2}\right)\left(k_{3} h_{4} k_{1}\right)-\left(k_{4} h_{1} k_{4}\right)\left(k_{2} h_{4} k_{3}\right)-\left(k_{2} h_{1} k_{3}\right)\left(k_{1} h_{4} k_{1}\right)\right]\right\}
\end{aligned}
$$

\subsubsection{Amplitud de dispersión a partir de supergravedad}

Partimos del lagrangiano

$$
\mathcal{L}=\frac{1}{2} \sqrt{-g}\left(\frac{1}{\kappa^{2}} R-g^{\mu \nu} \partial_{\mu} \phi \partial_{\nu} \phi\right)
$$

Introducimos la perturbación

$$
g_{\mu \nu}=\eta_{\mu \nu}+2 \kappa h_{\mu \nu}
$$

y expandimos a segundo orden

$$
\begin{aligned}
g^{\mu \nu} & \simeq \eta^{\mu \nu}-2 \kappa h^{\mu \nu}+4 \kappa^{2} h^{\mu \alpha} h_{\alpha}^{\nu} \\
\sqrt{-g} & \simeq 1+\kappa h+\frac{\kappa^{2}}{2}\left(h^{2}-2 h_{\mu \nu} h^{\mu \nu}\right)
\end{aligned}
$$

Expandimos ahora los términos de interacción que involucran al dilatón en el lagrangiano. Estos son

$$
\begin{gathered}
\mathcal{L}_{3}=\kappa\left[-\frac{1}{2} \eta^{\mu \nu} \partial^{\alpha} \phi \partial_{\alpha} \phi+\partial^{\mu} \phi \partial^{\nu} \phi\right] h_{\mu \nu} \\
\mathcal{L}_{4}=\kappa^{2} h_{\mu \nu} h_{\alpha \beta}\left[\left(-\frac{1}{4} \eta^{\mu \nu} \eta^{\alpha \beta}+\frac{1}{2} \eta^{\mu \alpha} \eta^{\nu \beta}\right) \partial_{\rho} \phi \partial^{\rho} \phi-2 \eta^{\alpha \nu} \partial^{\mu} \phi \partial^{\beta} \phi+\eta^{\mu \nu} \partial^{\alpha} \phi \partial^{\beta} \phi\right] .
\end{gathered}
$$

De 7.1 .22 podemos extraer el vértice

$$
V_{\phi \phi h}^{\mu \nu}\left(k_{1}, k_{2}\right)=i \kappa\left[-\eta^{\mu \nu}\left(k_{1} \cdot k_{2}\right)+k_{1}^{\mu} k_{2}^{\nu}+k_{2}^{\mu} k_{1}^{\nu}\right]
$$

y de 7.1 .23 extraemos

$$
V_{\phi \phi h h}^{\mu \nu, \alpha \beta}\left(k_{2}, k_{3}\right)=i \kappa^{2}\left[\left(-\eta^{\mu \nu} \eta^{\alpha \beta}+2 \eta^{\mu \alpha} \eta^{\nu \beta}\right)\left(k_{2} \cdot k_{3}\right)-4 \eta^{\alpha \nu}\left(k_{2}^{\mu} k_{3}^{\beta}+k_{3}^{\mu} k_{2}^{\beta}\right)+2\left(\eta^{\mu \nu} k_{2}^{\alpha} k_{3}^{\beta}+\eta^{\alpha \beta} k_{2}^{\mu} k_{3}^{\nu}\right)\right] .
$$


El propagador del gravitón es [84]

$$
D_{\mu \nu, \rho \sigma}(k)=-\frac{i}{2} \frac{1}{k^{2}-i \epsilon}\left(\eta_{\mu \rho} \eta_{\nu \sigma}+\eta_{\mu \sigma} \eta_{\nu \rho}-\frac{2}{D-2} \eta_{\mu \nu} \eta_{\rho \sigma}\right)
$$

mientras que el propagador del dilatón es

$$
D_{\phi}(k)=+\frac{i}{k^{2}-i \epsilon} .
$$

Expandiendo el término de gravedad del lagrangiano podemos extraer el vértice de tres gravitones que, en el caso de dos gravitones externos y luego de contraerlo con el propagador de un gravitón se escribe $[84]^{2}$

$$
\begin{aligned}
& h_{1 \mu \nu} h_{4 \alpha \beta} V_{h h h}^{\mu \nu, \alpha \beta, \rho \sigma}\left(k_{1}, k_{4},-k_{1}-k_{4}\right) D_{\rho \sigma, \gamma \delta}\left(k_{1}+k_{4}\right)= \\
& -2 \kappa \operatorname{Sym}\left[\left(h_{1} \cdot h_{4}\right)^{\gamma \delta}-\frac{1}{t}\left(\operatorname{Tr}\left(h_{1} \cdot h_{4}\right)\left[k_{1}^{\gamma} k_{1}^{\delta}+k_{1}^{\gamma} k_{4}^{\delta}+k_{4}^{\gamma} k_{4}^{\delta}\right]+\left(k_{1} \cdot h_{4} \cdot k_{1}\right) h_{1}^{\gamma \delta}\right.\right. \\
& \left.\left.+\left(k_{4} \cdot h_{1} \cdot k_{4}\right) h_{4}^{\gamma \delta}-2\left(k_{1} \cdot h_{4} \cdot h_{1}\right)^{\gamma} k_{1}^{\delta}-2\left(k_{4} \cdot h_{1} \cdot h_{4}\right)^{\gamma} k_{4}^{\delta}-2\left(k_{1} \cdot h_{4}\right)^{\gamma}\left(k_{4} \cdot h_{1}\right)^{\delta}\right)\right]
\end{aligned}
$$

Tenemos entonces tres vértices: $\phi \phi h, \phi \phi h h$ y $h h h$. Los diagramas relevantes para nuestro proceso son los mostrados en la Figura 7.2. Para el diagrama correspondiente al término de contacto obtenemos

$$
\begin{aligned}
A_{c}\left(k_{2}, k_{3}\right) & =h_{1 \mu \nu} h_{4 \alpha \beta} V_{\phi \phi h h}^{\mu \nu, \alpha \beta}\left(k_{2}, k_{3}\right) \\
& =-i \kappa^{2}\left[2\left(k_{2} \cdot k_{3}\right) \operatorname{Tr}\left(h_{1} \cdot h_{4}\right)-4 k_{2} \cdot h_{1} \cdot h_{4} \cdot k_{3}-4 k_{3} \cdot h_{1} \cdot h_{4} \cdot k_{2}\right] \\
& =i \kappa^{2}\left[t\left(h_{1} h_{4}\right)+4\left[\left(k_{2} h_{1} h_{4} k_{3}\right)+\left(k_{3} h_{1} h_{4} k_{2}\right)\right]\right],
\end{aligned}
$$

donde hemos utilizado las definiciones de (7.1.11) y

$$
k_{i}^{2}=0 ; k_{1}+k_{2}+k_{3}+k_{4}=0 .
$$

Para el diagrama correspondiente al polo del gravitón en el canal $t$, obtenemos

$$
\begin{aligned}
A_{t}\left(k_{2}, k_{3}\right)= & h_{1 \mu \nu} h_{4 \alpha \beta} V_{h h h}^{\mu \nu, \alpha \beta, \rho \sigma}\left(k_{1}, k_{4},-k_{1}-k_{4}\right) D_{\rho \sigma, \gamma \delta}\left(k_{1}+k_{4}\right) V_{\phi \phi h}^{\gamma \delta}\left(k_{2}, k_{3}\right) \\
= & -i 2 \kappa^{2}\left[\left(h_{1} \cdot h_{4}\right)^{\gamma \delta}-\frac{1}{t}\left(\operatorname{Tr}\left(h_{1} \cdot h_{4}\right)\left[k_{1}^{\gamma} k_{1}^{\delta}+k_{1}^{\gamma} k_{4}^{\delta}+k_{4}^{\gamma} k_{4}^{\delta}\right]+\left(k_{1} \cdot h_{4} \cdot k_{1}\right) h_{1}^{\gamma \delta}\right.\right. \\
& \left.\left.+\left(k_{4} \cdot h_{1} \cdot k_{4}\right) h_{4}^{\gamma \delta}-2\left(k_{1} \cdot h_{4} \cdot h_{1}\right)^{\gamma} k_{1}^{\delta}-2\left(k_{4} \cdot h_{1} \cdot h_{4}\right)^{\gamma} k_{4}^{\delta}-2\left(k_{1} \cdot h_{4}\right)^{\gamma}\left(k_{4} \cdot h_{1}\right)^{\delta}\right)\right] \\
& {\left[-\eta^{\gamma \delta}\left(k_{2} \cdot k_{3}\right)+k_{2}^{\gamma} k_{3}^{\delta}+k_{3}^{\gamma} k_{2}^{\delta}\right] } \\
= & \frac{i \kappa^{2}}{t}\left\{\frac{\left(h_{1} h_{4}\right)}{2}\left[-3 t^{2}+4 s u+s^{2}+u^{2}\right]\right. \\
& +2\left[u\left(\left(k_{2} h_{1} h_{4} k_{1}\right)+\left(k_{4} h_{1} h_{4} k_{3}\right)\right)+s\left(\left(k_{3} h_{1} h_{4} k_{1}\right)+\left(k_{4} h_{1} h_{4} k_{2}\right)\right)\right. \\
& \left.-t\left(\left(k_{2} h_{1} h_{4} k_{3}\right)+\left(k_{3} h_{1} h_{4} k_{2}\right)+\left(k_{4} h_{1} h_{4} k_{1}\right)\right)\right] \\
& \left.+4\left[\left(k_{2} h_{1} k_{3}\right)\left(k_{1} h_{4} k_{1}\right)+\left(k_{4} h_{1} k_{4}\right)\left(k_{2} h_{4} k_{3}\right)-\left(k_{4} h_{1} k_{2}\right)\left(k_{3} h_{4} k_{1}\right)-\left(k_{4} h_{1} k_{3}\right)\left(k_{2} h_{4} k_{1}\right)\right]\right\} .
\end{aligned}
$$

\footnotetext{
${ }^{2}$ Hemos sustituido $\kappa \rightarrow 2 \kappa$ con respecto a la referencia, pues en esta la perturbación se escribe $g^{\mu \nu}=$ $\eta^{\mu \nu}+\kappa h^{\mu \nu}$ en lugar de $g^{\mu \nu}=\eta^{\mu \nu}+2 \kappa h^{\mu \nu}$ como aquí.
} 
Reescribimos el primer término como

$$
-3 t^{2}+4 s u+s^{2}+u^{2}=-3 t^{2}+2\left[(s+u)^{2}-s^{2}-u^{2}\right]+s^{2}+u^{2}=-t^{2}-s^{2}-u^{2},
$$

pues $s+t+u=0$. Debido a la conservación del momento total en el vértice podemos reescribir el segundo término

$$
\begin{aligned}
& u\left(\left(k_{2} h_{1} h_{4} k_{1}\right)+\left(k_{4} h_{1} h_{4} k_{3}\right)\right)+s\left(\left(k_{3} h_{1} h_{4} k_{1}\right)+\left(k_{4} h_{1} h_{4} k_{2}\right)\right) \\
& \left.-t\left(\left(k_{2} h_{1} h_{4} k_{3}\right)+\left(k_{3} h_{1} h_{4} k_{2}\right)+\left(k_{4} h_{1} h_{4} k_{1}\right)\right)\right]= \\
& -u\left(\left(k_{3} h_{1} h_{4} k_{1}\right)+\left(k_{4} h_{1} h_{4} k_{2}\right)\right)-s\left(\left(k_{2} h_{1} h_{4} k_{1}\right)+\left(k_{4} h_{1} h_{4} k_{3}\right)\right) \\
& -t\left(\left(k_{2} h_{1} h_{4} k_{3}\right)+\left(k_{3} h_{1} h_{4} k_{2}\right)-\left(k_{4} h_{1} h_{4} k_{1}\right)\right)
\end{aligned}
$$

donde nuevamente utilizamos $s+t+u=0$. Con esto, la expresión final para este diagrama es

$$
\begin{aligned}
A_{t}\left(k_{2}, k_{3}\right)= & -\frac{i \kappa^{2}}{t}\left\{\frac{\operatorname{Tr}\left(h_{1} \cdot h_{4}\right)}{2}\left[t^{2}+s^{2}+u^{2}\right]\right. \\
& +2\left[-u\left(\left(k_{3} h_{1} h_{4} k_{1}\right)+\left(k_{4} h_{1} h_{4} k_{2}\right)\right)-s\left(\left(k_{2} h_{1} h_{4} k_{1}\right)+\left(k_{4} h_{1} h_{4} k_{3}\right)\right)\right. \\
& \left.-t\left(\left(k_{2} h_{1} h_{4} k_{3}\right)+\left(k_{3} h_{1} h_{4} k_{2}\right)-\left(k_{4} h_{1} h_{4} k_{1}\right)\right)\right] \\
& +4\left(\left(k_{4} h_{1} k_{2}\right)\left(k_{3} h_{4} k_{1}\right)+\left(k_{4} h_{1} k_{3}\right)\left(k_{2} h_{4} k_{1}\right)-\left(k_{2} h_{1} k_{3}\right)\left(k_{1} h_{4} k_{1}\right)-\left(k_{4} h_{1} k_{4}\right)\left(k_{2} h_{4} k_{3}\right)\right\} .
\end{aligned}
$$

Para el diagrama correspondiente al polo del dilatón en el canal $s$, obtenemos

$$
\begin{aligned}
A_{s}\left(k_{2}, k_{3}\right) & =h_{1 \mu \nu} h_{4 \alpha \beta} V_{\phi \phi h}^{\mu \nu}\left(k_{2},-k_{1}-k_{2}\right) D_{\phi}\left(k_{1}+k_{2}\right) V_{\phi \phi h}^{\alpha \beta}\left(k_{1}+k_{2},-k_{3}\right) \\
& =-\frac{4 i \kappa^{2}}{s}\left(k_{2} h_{1} k_{2}\right)\left(k_{3} h_{4} k_{3}\right) .
\end{aligned}
$$

Análogamente, para el diagrama d), correspondiente al polo del dilatón en el canal $u$,

$$
\begin{aligned}
A_{u}\left(k_{2}, k_{3}\right) & =h_{1 \mu \nu} h_{4 \alpha \beta} V_{\phi \phi h}^{\mu \nu}\left(k_{3},-k_{1}-k_{3}\right) D_{\phi}\left(k_{1}+k_{3}\right) V_{\phi \phi h}^{\alpha \beta}\left(k_{1}+k_{3},-k_{2}\right) \\
& =-\frac{4 i \kappa^{2}}{u}\left(k_{3} h_{1} k_{3}\right)\left(k_{2} h_{4} k_{2}\right) .
\end{aligned}
$$

Sumando los cuatro diagramas, la amplitud de cuatro puntos a nivel árbol es

$$
\begin{aligned}
A_{4}= & A_{a}+A_{t}+A_{s}+A_{u} \\
= & \frac{i \kappa^{2}}{t}\left\{\frac{\left(h_{1} h_{4}\right)}{2}\left[t^{2}-s^{2}-u^{2}\right]\right. \\
& -2\left[u\left(\left(k_{3} h_{1} h_{4} k_{1}\right)+\left(k_{4} h_{1} h_{4} k_{2}\right)\right)+s\left(\left(k_{2} h_{1} h_{4} k_{1}\right)+\left(k_{4} h_{1} h_{4} k_{3}\right)\right)\right. \\
& \left.-t\left(\left(k_{2} h_{1} h_{4} k_{3}\right)+\left(k_{3} h_{1} h_{4} k_{2}\right)+\left(k_{4} h_{1} h_{4} k_{1}\right)\right)\right]+4\left(\left(k_{2} h_{1} k_{3}\right)\left(k_{1} h_{4} k_{1}\right)+\left(k_{4} h_{1} k_{4}\right)\left(k_{2} h_{4} k_{3}\right)\right. \\
& \left.\left.-\left(k_{4} h_{1} k_{2}\right)\left(k_{3} h_{4} k_{1}\right)-\left(k_{4} h_{1} k_{3}\right)\left(k_{2} h_{4} k_{1}\right)-\frac{t}{s}\left(k_{2} h_{1} k_{2}\right)\left(k_{3} h_{4} k_{3}\right)-\frac{t}{u}\left(k_{3} h_{1} k_{3}\right)\left(k_{2} h_{4} k_{2}\right)\right)\right\} .
\end{aligned}
$$

De la última expresión, y multiplicando por stu obtenemos

$$
\begin{aligned}
s t u A_{4}= & i \kappa^{2}\left\{s u \left[\frac{1}{2}\left(h_{1} h_{4}\right)\left[t^{2}-s^{2}-u^{2}\right]\right.\right. \\
& +2\left[t\left(\left(k_{2} h_{1} h_{4} k_{3}\right)+\left(k_{3} h_{1} h_{4} k_{2}\right)+\left(k_{4} h_{1} h_{4} k_{1}\right)\right)\right. \\
& \left.\left.-u\left(\left(k_{3} h_{1} h_{4} k_{1}\right)+\left(k_{4} h_{1} h_{4} k_{2}\right)\right)-s\left(\left(k_{2} h_{1} h_{4} k_{1}\right)+\left(k_{4} h_{1} h_{4} k_{3}\right)\right)\right]\right] \\
& \left.+4\left(2 s u\left(k_{2} h_{1} k_{3}\right)\left(k_{2} h_{4} k_{3}\right)+u^{2}\left(k_{2} h_{1} k_{2}\right)\left(k_{3} h_{4} k_{3}\right)+s^{2}\left(k_{3} h_{1} k_{3}\right)\left(k_{2} h_{4} k_{2}\right)\right)\right\} 7
\end{aligned}
$$

que coincide exactamente con la amplitud de cuatro cuerdas cerradas a nivel árbol. Hemos utilizado la identidad (7.1.16 proveniente de la conservación del momento total. 


\subsubsection{Lagrangiano efectivo}

Hasta ahora hemos calculado la amplitud de cuatro cuerdas cerradas -dos gravitones y dos dilatones- para el régimen $0<\alpha^{\prime} \tilde{t} \ll 1 \ll \alpha^{\prime} \tilde{s}$ de dos maneras distintas (desde la teoría de cuerdas y desde supergravedad). Ahora queremos escribir una teoría de campos efectiva $\mathcal{L}_{\text {eff }}$ que reproduzca estos mismos resultados, a fin de calcular el tensor hadrónico.

A partir de supergravedad podemos construir 6 términos que involucran dos gravitones y dos dilatones, pidiendo que cada uno de estos términos esté derivado una sola vez y sin trazas ni divergencias para los gravitones. Estos son

$$
\begin{aligned}
\mathcal{L}_{a} & =\partial_{M} \Phi \partial^{M} \Phi\left[\partial^{N} h^{R P} \partial_{N} h_{R P}\right] \\
\mathcal{L}_{b} & =\partial_{M} \Phi \partial^{M} \Phi\left[\partial^{N} h^{R P} \partial_{P} h_{R N}\right] \\
\mathcal{L}_{c} & =\partial_{M} \Phi \partial^{N} \Phi\left[\partial^{M} h^{R P} \partial_{N} h_{R P}\right] \\
\mathcal{L}_{d} & =\partial_{M} \Phi \partial^{N} \Phi\left[\partial^{M} h^{R P} \partial_{R} h_{P N}\right] \\
\mathcal{L}_{e} & =\partial_{M} \Phi \partial^{N} \Phi\left[\partial^{R} h^{M P} \partial_{R} h_{N P}\right] \\
\mathcal{L}_{f} & =\partial_{M} \Phi \partial^{N} \Phi\left[\partial^{R} h^{M P} \partial_{P} h_{R N}\right]
\end{aligned}
$$

Con esto, todos los lagrangianos que podemos construir de esta manera son de la forma

$$
\mathcal{L}=a \mathcal{L}_{a}+b \mathcal{L}_{b}+c \mathcal{L}_{c}+d \mathcal{L}_{d}+\mathcal{L}_{e}+f \mathcal{L}_{f}
$$

Imponemos ahora la forma para el gravitón 6.1.11

$$
h^{M N}=\frac{1}{2}\left(A^{M} v^{N}+v^{M} A^{N}\right)
$$

Con esto, calculamos los términos, resultando

$$
\begin{aligned}
\mathcal{L}_{a} & =\frac{1}{2} \partial_{M} \Phi \partial^{M} \Phi v_{a} v^{a} \partial^{m} A^{n} \partial_{m} A_{n} \\
\mathcal{L}_{b} & =\frac{1}{4} \partial_{M} \Phi \partial^{M} \Phi v_{a} v^{a} \partial^{m} A^{n} \partial_{n} A_{m} \\
\mathcal{L}_{c} & =\frac{1}{2} \partial_{m} \Phi \partial^{p} \Phi v_{a} v^{a} \partial^{m} A^{n} \partial_{p} A_{n} \\
\mathcal{L}_{d} & =\frac{1}{4} \partial_{m} \Phi \partial^{p} \Phi v_{a} v^{a} \partial^{m} A^{n} \partial_{n} A_{p} \\
\mathcal{L}_{e} & =\frac{1}{4} \partial_{m} \Phi \partial^{p} \Phi v_{a} v^{a} \partial^{n} A^{m} \partial_{n} A_{p}+\frac{1}{4} \partial_{a} \Phi \partial^{b} \Phi v^{a} v_{b} \partial^{n} A^{m} \partial_{n} A_{m} \\
\mathcal{L}_{f} & =\frac{1}{4} \partial_{a} \Phi \partial^{b} \Phi v^{a} v_{b} \partial^{m} A^{n} \partial_{n} A_{m}
\end{aligned}
$$

La contribución de cada término de este lagrangiano efectivo a la amplitud de cuatro cuerdas 
cerradas es

$$
\begin{aligned}
\mathcal{L}_{a} & \rightarrow 2\left(k_{2} k_{3}\right) 2\left(k_{1} k_{4}\right) \operatorname{Tr}\left(h_{1} h_{4}\right)=t^{2}\left(h_{1} h_{4}\right) \\
\mathcal{L}_{b} & \rightarrow 2\left(k_{2} k_{3}\right) 2\left(k_{4} h_{1} h_{4} k_{1}\right)=-2 t\left(k_{4} h_{1} h_{4} k_{1}\right) \\
\mathcal{L}_{c} & \rightarrow 2 \operatorname{Tr}\left(h_{1} h_{4}\right)\left[\left(k_{2} k_{1}\right)\left(k_{2} k_{4}\right)+\left(k_{2} k_{4}\right)\left(k_{3} k_{1}\right)\right]=\frac{1}{2}\left(s^{2}+u^{2}\right)\left(h_{1} h_{4}\right) \\
\mathcal{L}_{d} & \rightarrow\left(k_{2} k_{1}\right)\left(k_{4} h_{1} h_{4} k_{3}\right)+\left(k_{3} k_{1}\right)\left(k_{4} h_{1} h_{4} k_{2}\right)+\left(k_{2} k_{4}\right)\left(k_{1} h_{4} h_{1} k_{3}\right)+\left(k_{3} k_{4}\right)\left(k_{1} h_{4} h_{1} k_{2}\right) \\
& =-\frac{1}{2}\left[s\left(\left(k_{4} h_{1} h_{4} k_{3}\right)+\left(k_{2} h_{1} h_{4} k_{1}\right)\right)+u\left(\left(k_{4} h_{1} h_{4} k_{2}\right)+\left(k_{3} h_{1} h_{1} k_{3}\right)\right)\right] \\
\mathcal{L}_{e} & \rightarrow 2\left(k_{1} k_{4}\right)\left(k_{2} h_{1} h_{4} k_{3}\right)+2\left(k_{1} k_{4}\right)\left(k_{3} h_{1} h_{4} k_{2}\right)=-t\left(\left(k_{2} h_{1} h_{4} k_{3}\right)+\left(k_{3} h_{1} h_{4} k_{2}\right)\right) \\
\mathcal{L}_{f} & \rightarrow\left(k_{2} h_{1} k_{4}\right)\left(k_{3} h_{4} k_{1}\right)+\left(k_{3} h_{1} k_{4}\right)\left(k_{2} h_{4} k_{3}\right)+\left(k_{2} h_{4} k_{1}\right)\left(k_{3} h_{1} k_{4}\right)+\left(k_{3} h_{4} k_{1}\right)\left(k_{2} h_{1} k_{4}\right) \\
& =2\left(\left(k_{2} h_{1} k_{4}\right)\left(k_{3} h_{4} k_{1}\right)+\left(k_{3} h_{1} k_{4}\right)\left(k_{2} h_{4} k_{1}\right)\right)
\end{aligned}
$$

Igualando las amplitudes con las obtenidas en las Secciones 7.1.1 y 7.1.2 vemos que el lagrangiano efectivo es

$$
\mathcal{L}_{e f f} \propto \frac{1}{4}\left[2 v^{i} v_{i} \partial_{m} \Phi \partial^{p} \Phi F^{m n} F_{p n}+v^{i} v^{j} \partial_{i} \Phi \partial_{j} \Phi F^{m n} F_{m n}\right],
$$

donde definimos $F_{m n}=\partial_{m} A_{n}-\partial_{n} A_{m}$. Es fácil ver que hay un error en la ecuación (82) de la referencia [5]. $]^{3}$ Sin embargo el primer término, que es el dominante, es correcto (a menos de un factor global) por lo que el resultado queda prácticamente inalterado.

\subsubsection{Cálculo del tensor hadrónico}

Tenemos entonces el lagrangiano

$$
\left.K\right|_{\tilde{t}=0}=\frac{1}{8} \int d^{10} x\left\{2 v^{i} v_{i} \partial_{m} \Phi \partial^{p} \Phi F^{m n} F_{p n}+v^{i} v^{j} \partial_{i} \Phi \partial_{j} \Phi F^{m n} F_{m n}\right\},
$$

El dilatón está canónicamente normalizado y el campo de gauge tiene la normalización definida en (6.1.11). A partir de esta acción efectiva será sencillo pasar nuestro cálculo en espacio plano a uno en espacio curvo.

Para $x$ pequeño $\left(0<\alpha^{\prime} t \ll 1 \ll \alpha^{\prime} s\right)$ el primer término en esta acción dominará, pues como hemos visto su contribución será cuadrática en $s$. Si evaluamos este lagrangiano efectivo en el elemento de matriz de $A d S$ obtendremos la contribución de cuerdas excitadas a la parte imaginaria de la dispersión Compton de interés. Como dijimos antes, para la región $\mathbf{B}$, en la que

\footnotetext{
${ }^{3}$ Corresponde a la ecuación (5.7) en la versión publicada de [5] . El lagrangiano que aparece allí es

$$
\left.K\right|_{\tilde{t}=0}=\frac{1}{8} \int d^{10} x\left\{4 v^{i} v_{i} \partial_{m} \Phi F^{m n} F_{p n} \partial^{p} \Phi-\left(\partial^{M} \Phi \partial_{M} \Phi v^{i} v_{i}+2 v^{i} \partial_{i} \Phi v^{j} \partial_{j} \Phi\right) F_{m n} F^{m n}\right\},
$$

El error consiste en que el segundo término de aquí no aparece y el tercero tiene el signo incorrecto. 
$x$ no es exponencialmente pequeño, podemos olvidarnos del factor $\tilde{s}^{\alpha^{\prime} \tilde{t}}$ con lo que resulta ${ }^{4}$

$$
\begin{aligned}
n_{\mu} n_{\nu} \operatorname{Im}_{\mathrm{exc}} \mathrm{T}^{\mu \nu} & =\left.(K \operatorname{Im} G)\right|_{\tilde{t}=0} \\
& =\frac{\pi \alpha^{\prime}}{2} \sum_{m=1}^{\infty} \int d^{6} x_{\perp} \sqrt{-g} v^{a} v_{a} \partial_{m} \Phi_{\mathrm{i}}^{*} F^{m n}(-q) \delta\left(m-\alpha^{\prime} \tilde{s} / 4\right) F_{p n}(q) \partial^{p} \Phi_{\mathrm{i}}
\end{aligned}
$$

Todas las contracciones están hechas aquí con la métrica completa en diez dimensiones. El invariante $\tilde{s}$ involucra en principio un operador diferencial actuando sobre los campos a su derecha,

$$
\alpha^{\prime} \tilde{s}=\frac{\alpha^{\prime} s R^{2}}{r^{2}}+\frac{\alpha^{\prime}}{R^{2}}\left(r^{2} \partial_{r}^{2}+5 r \partial_{r}+\hat{\nabla}_{W}^{2}\right) .
$$

Todas las funciones son suaves en la dirección radial, con lo que $r \partial_{r}=O(1)$; también suponemos que el laplaciano adimensional en $W$ es de orden $O(1)$. Con esto, $\alpha^{\prime} \tilde{s}=\alpha^{\prime} s\left(R^{2} / r^{2}\right)$ más correcciones de orden $O\left(\alpha^{\prime} / R^{2} \sim(\lambda)^{-1 / 2}\right)$, que para cuerdas excitadas puede despreciarse con respecto al entero $m$ en la función delta. Esto es consistente con nuestro argumento de que la interacción efectiva es local en la escala del $A d S$. El caso $m=0$ no está cubierto por este argumento, pero es lo que hemos calculado en la Sección anterior. La función delta queda entonces $\delta\left(m-\alpha^{\prime} s R^{2} / 4 r^{2}\right)$, con lo que sirve para fijar la integración en la coordenada radial.

Podemos ahora evaluar la amplitud (7.1.58). Escribiremos aquí los resultados principales de [5] , dejando el detalle del cálculo para la Subsección 7.2.4, que es equivalente. Elegimos los momentos para todas las partículas como entrantes, como se ve en la Figura 7.3 Estamos utilizando las soluciones

$$
\begin{aligned}
\Phi_{i}^{\ell}(P) & =c_{i}\left(\frac{r}{r_{0}}\right)^{-\Delta} e^{i P \cdot y} Y^{\ell}\left(S^{p-4}\right) \quad ; \quad r_{0}=\Lambda R^{2} \\
A_{\mu}(q) & =n_{\mu} e^{i q \cdot y} \frac{q R^{2}}{r} K_{1}\left(\frac{q R^{2}}{r}\right), \\
A_{r}(q) & =-i(q \cdot n) e^{i q \cdot y} \frac{R^{4}}{r^{3}} K_{0}\left(\frac{q R^{2}}{r}\right) \\
& =-\frac{i}{q^{2}} \eta^{\mu \nu} q_{\mu} \partial_{r} A_{\nu},
\end{aligned}
$$

${ }^{4}$ Nos interesa el factor $m^{\alpha^{\prime} \tilde{t} / 2}=\left(\frac{\alpha^{\prime} \tilde{s}}{4}\right)^{\alpha^{\prime} \tilde{t} / 2}$. En este límite

$$
\alpha^{\prime} \tilde{t} \sim \lambda^{-1 / 2} \quad ; \quad \alpha^{\prime} \tilde{s}=\alpha^{\prime} \frac{R^{2}}{r_{i n t}} s=\alpha^{\prime} \frac{R^{2}}{r_{i n t}} q^{2}\left(\frac{1}{x}-1+t\right) \simeq \frac{\alpha^{\prime}}{R^{2}} \frac{1}{x}
$$

donde usamos $r_{\text {int }} \sim q R^{2}$ y $x \ll 1$.

La región $\mathrm{B}$ es

$$
e^{-\sqrt{\lambda}} \ll x \ll \frac{1}{\sqrt{\lambda}} \ll 1 \quad ; \quad \frac{\alpha^{\prime}}{R^{2}} \sim \frac{1}{\sqrt{\lambda}},
$$

que es equivalente a

$$
1 \ll\left(\frac{\alpha^{\prime} \tilde{s}}{4}\right)^{\alpha^{\prime} \tilde{t} / 2} \ll\left(\frac{e^{\sqrt{\lambda}}}{4 \sqrt{\lambda}}\right)^{1 / 2 \sqrt{\lambda}} \rightarrow e^{1 / 2} \sim 1,65 \quad(\lambda \rightarrow \infty)
$$

Es decir, demostramos que $\left(\frac{\alpha^{\prime} \tilde{s}}{4}\right)^{\alpha^{\prime} \tilde{t} / 2} \sim 1$. 


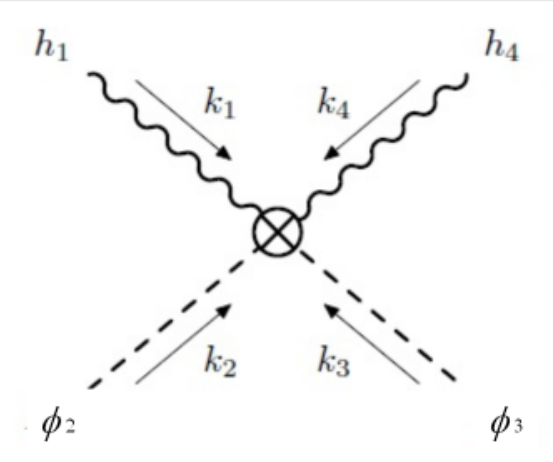

Figura 7.3: Interacción efectiva de cuatro puntos. Las líneas onduladas representan gravitones físicos y las líneas de trazos dilatones.

Las componentes del tensor de campo son

$$
\begin{gathered}
F_{\mu \nu}(q)=i\left(q_{\mu} n_{\nu}-n_{\mu} q_{\nu}\right) \frac{q R^{2}}{r} K_{1}\left(q R^{2} / r\right) e^{i q \cdot y} \\
F_{\mu r}(q)=-\left(n_{\mu} q^{2}-q_{\mu} q \cdot n\right) \frac{R^{4}}{r^{3}} K_{0}\left(q R^{2} / r\right) e^{i q \cdot y} .
\end{gathered}
$$

lo que implica

$$
\begin{gathered}
F_{\mu p} F_{\nu}{ }^{p}=\left(n_{\mu}-q_{\mu} \frac{q \cdot n}{q^{2}}\right)\left(n_{\nu}-q_{\nu} \frac{q \cdot n}{q^{2}}\right) \frac{w^{4}}{R^{2}}\left[K_{0}^{2}(w)+K_{1}^{2}(w)\right] \\
+q_{\mu} q_{\nu}\left(n^{2}-\frac{q \cdot n q \cdot n}{q^{2}}\right) \frac{w^{4}}{q^{2} R^{2}} K_{1}^{2}(w)
\end{gathered}
$$

donde $w=q R^{2} / r 5^{5}$ Entonces,

$$
\begin{array}{r}
n_{\mu} n_{\nu} \operatorname{Im}_{\mathrm{exc}} \mathrm{T}^{\mu \nu}=\frac{\pi \rho\left|c_{\mathrm{i}}\right|^{2}}{8}\left(\frac{\Lambda^{2}}{q^{2}}\right)^{\Delta-1} \sum_{m=1}^{\infty} w_{m}^{2 \Delta+2}\left\{\frac{1}{x} K_{1}^{2}\left(w_{m}\right)\left(n^{2}-\frac{q \cdot n q \cdot n}{q^{2}}\right)\right. \\
\left.+\frac{4 x}{q^{2}}\left[K_{0}^{2}\left(w_{m}\right)+K_{1}^{2}\left(w_{m}\right)\right]\left(P \cdot n-\frac{P \cdot q n \cdot q}{q^{2}}\right)^{2}\right\}
\end{array}
$$

donde $w_{m}=q R^{2} / r_{m}$ y $r_{m}=R\left(\alpha^{\prime} s\right)^{1 / 2} / 2 m^{1 / 2}$. La constante adimensional $\rho$ proviene de la integral angular ${ }^{6}$

$$
\int d^{5} \Omega \sqrt{\hat{g}_{W}} v^{a} v_{a}|Y|^{2}=\rho R^{2}
$$

Las funciones de estructura son entonces

$$
\begin{aligned}
& F_{1}=\frac{\pi^{2} \rho\left|c_{\mathrm{i}}\right|^{2}}{4 x}\left(\frac{\Lambda^{2}}{q^{2}}\right)^{\Delta-1} \sum_{m=1}^{\infty} w_{m}^{2 \Delta+2} K_{1}^{2}\left(w_{m}\right), \\
& F_{2}=\frac{\pi^{2} \rho\left|c_{\mathrm{i}}\right|^{2}}{2}\left(\frac{\Lambda^{2}}{q^{2}}\right)^{\Delta-1} \sum_{m=1}^{\infty} w_{m}^{2 \Delta+2}\left[K_{0}^{2}\left(w_{m}\right)+K_{1}^{2}\left(w_{m}\right)\right] .
\end{aligned}
$$

\footnotetext{
${ }^{5}$ Las contribuciones de $F_{r p} F_{\nu}^{p}$ y $F_{r p} F_{r}{ }^{p}$ son despreciables.

${ }^{6}$ Notemos que $v^{a}$ y $\partial_{a} \psi$ son independientes de $R$.
} 
El argumento de las funciones de Bessel es $w_{m} \sim x^{1 / 2} m^{1 / 2}(\lambda)^{1 / 4}$ y por esto cuando $x \ll$ $(\lambda)^{-1 / 2}$ las sumas pueden aproximarse por integrales. Usando

$$
I_{j, n}=\int_{0}^{\infty} d w w^{n} K_{j}^{2}(w)=2^{n-2} \frac{\Gamma(\nu+j) \Gamma(\nu-j) \Gamma(\nu)^{2}}{\Gamma(2 \nu)}, \quad \nu=\frac{1}{2}(n+1) .
$$

de manera que $I_{1, n}=\frac{n+1}{n-1} I_{0, n}$. Encontramos

$$
\begin{aligned}
& F_{1}=\frac{1}{x^{2}}\left(\frac{\Lambda^{2}}{q^{2}}\right)^{\Delta-1} \frac{\pi^{2} \rho\left|c_{\mathrm{i}}\right|^{2}}{4(4 \pi \lambda)^{1 / 2}} I_{1,2 \Delta+3}, \\
& F_{2}=2 x \frac{2 \Delta+3}{\Delta+2} F_{1} .
\end{aligned}
$$

Notar que en este régimen $F_{1} \neq 0$, a diferencia de lo que ocurría en la región A. Esto se debe a que en la presente región estamos estudiando la estructura interna del blanco y no a este como un todo.

\section{2. $\quad$ DIS desde mesones escalares}

Estudiaremos ahora el caso de DIS donde el blanco es un mesón escalar. A partir de esta Sección, y por el resto del Capítulo, presentamos resultados originales, publicados en [3].

En el caso de DIS de mesones escalares seguimos factorizando los gravitones como $h^{M N}=$ $(1 / 2)\left(A^{M} v^{N}+A^{N} v^{M}\right)$ donde $A^{m}$ es nuevamente el campo de gauge $U(1)$ que se propaga en el interior del $A d S_{5}$. Para el modelo D3D7, $v^{i}$ es un vector de Killing constante en la $S^{3}$. ${ }^{7}$ En la teoría de supercuerdas estos gravitones corresponden a cuerdas cerradas, mientras que los mesones escalares están representados por cuerdas abiertas con sus extremos sobre la D7brana de sabor. Debemos calcular entonces la amplitud de dispersión de cuatro puntos entre dos cuerdas abiertas y dos cerradas, $\mathcal{A}_{4}^{202 c, s c a l a r}$, donde los supraíndices $o$ y $c$ representan cuerdas abiertas (del inglés open) y cerradas (closed) respectivamente. Esta amplitud puede expresarse como una suma de dos términos, cada uno de los cuales se compone de un factor cinemático $\mathcal{K}_{i}^{202 c, s c a l a r}$ y de un prefactor $\mathcal{P}_{i}^{202 c, s c a l a r}$ que tiene la estructura usual de funciones gamma, y aporta la dependencia en $\alpha^{\prime}$ :

$$
\mathcal{A}_{4}^{202 c, \text { scalar }}=\mathcal{P}_{1}^{202 c, \text { scalar }} \mathcal{K}_{1}^{202 c, \text { scalar }}+\mathcal{P}_{2}^{202 c, \text { scalar }} \mathcal{K}_{2}^{202 c, \text { scalar }}
$$

En el régimen cinemático en que estamos interesados solo nos interesa el primer término, pues es el que posee el polo en el canal $t$ [85].

Nos interesa el tensor hadrónico, por lo que tenemos que calcular la parte imaginaria de la dispersión Compton. En particular, tenemos que evaluar $\mathcal{K}_{1}^{202 c, \text { scalar }}$ en $\tilde{t}=0$, lo que puede hacerse, al igual que en el caso de los glueballs, de dos formas:

- (i) Tomando la amplitud $\mathcal{A}_{4}^{202 c, \text { scalar }}$ y reemplazando la cuerda cerrada por el gravitón $h^{m i}=A^{m} v^{i}$, y la cuerda abierta por la fluctuación escalar $X$, con lo que se construye el lagrangiano de interacción efectivo de cuatro puntos $\mathcal{L}_{h h X X}^{e f f}$ con el que puede calcularse el tensor hadrónico.

\footnotetext{
${ }^{7}$ Para los modelos D4D6 $\overline{\mathrm{D} 6}$ y $\mathrm{D} 4 \mathrm{D} 8 \overline{\mathrm{D} 8}, v^{i}$ será un vector de Killing constante sobre $S^{2}$ y $S^{4}$ respectivamente.
} 
- (ii) Teniendo en cuenta que el lagrangiano efectivo $\mathcal{L}_{h h X X}^{e f f}$ viene dado por el coeficiente para el polo del gravitón en el canal $t$ multiplicado por el prefactor $\mathcal{P}$ que contiene la dependencia en $\alpha^{\prime}$. Esta segunda opción puede verse como el cálculo para bajas energías de la amplitud de dispersión (i.e. en el límite $\alpha^{\prime} \rightarrow 0$ ). Para hacer este cálculo hace falta considerar el propagador del gravitón y el vértice de tres puntos de gravitones, provenientes de supergravedad IIB, así como el vértice de un gravitón y dos campos escalares derivado de la acción de DBI con fluctuaciones escalares en la brana de sabor.

En el presente caso discutiremos el método (i), proveniente de teoría de cuerdas, en la Subsección 7.2.1 y el método (ii), proveniente de supergravedad, en la Subsección 7.2.2. Veremos que, como era de esperarse, ambos resultados para la amplitud de cuatro puntos coinciden. También veremos que este resultado es muy similar al obtenido por Polchinski y Strassler en [5] para el caso de glueballs descripto en la Sección anterior. En la Subsección 7.2.3 calcularemos el lagrangiano efectivo para este caso mientras que en la Subsección 7.2.4 agregaremos la curvatura para calcular el tensor hadrónico y las funciones de estructura. El caso de $x$ exponencialmente pequeño para mesones escalares será postergado hasta la Sección 7.5 .

A partir de aquí, tanto los momentos $k_{a}$ de los campos como las polarizaciones de los gravitones $h$ y las polarizaciones de los mesones vectoriales $\epsilon$ (en la Sección 7.3) son paralelos a las direcciones de la D7-brana de sabor. Los mesones escalares, en cambio, representan fluctuaciones perpendiculares a esta.

\subsubsection{Amplitud de dispersión de cuatro puntos para cuerdas abiertas y cerradas}

Para obtener la amplitud de dispersión a nivel árbol de dos cuerdas cerradas (gravitones) y dos cuerdas abiertas (mesones escalares o vectoriales), tenemos que plantear una integral funcional con las inserciones de operadores de vértice en la hoja de mundo correspondientes a los estados asintóticos de las cuerdas a dispersarse. En [85] se muestra que una amplitud mixta de cuerdas abiertas y cerradas puede mapearse en otra de solamente cuerdas abiertas 8

La superficie de hoja de mundo correspondiente a la amplitud mixta de cuerdas abiertas y cerradas es equivalente al disco unitario $D=\{z \in \mathbb{C}|| z \mid \leq 1\}$. Utilizando la transformación de Möbius $z \rightarrow i(1+z)(1-z)^{-1}$ este disco se puede mapear conformemente al semiplano complejo superior $\mathbf{H}_{+}=\{z \in \mathbb{C} \mid \operatorname{Im}(z) \geq 0\}$. Los operadores de vértice correspondientes a cuerdas abiertas $V_{o}\left(x_{i}\right)$, estarán insertados en posiciones $x_{i} \in \mathbf{R}$ del borde del disco $D$, al cual estará adherido el volumen de mundo de la $\mathrm{D} p$-brana. Las cuerdas cerradas, en cambio, se propagan en el interior del espaciotiempo de diez dimensiones, por lo que sus operadores de vértice $V_{c}\left(\bar{z}_{i}, z_{i}\right)$ estarán insertados en puntos $z_{i} \in \mathbb{C}$, correspondientes al interior del disco $D$. en la Figura 7.4 se muestra la topología en teoría de cuerdas de los distintos diagramas de Feynman.

Los operadores de vértice para las cuerdas abiertas son 9

$$
\begin{aligned}
V_{o}^{(-1)}\left(x, \epsilon_{\mu}, p\right) & =g_{o} e^{-\phi} \epsilon_{\mu} \psi^{\mu} e^{i p \cdot X}(x), \\
V_{o}^{(0)}\left(x, \epsilon_{\mu}, p\right) & =g_{o}\left(2 \alpha^{\prime}\right)^{-1 / 2} \epsilon_{\mu}\left(i \partial_{x} X^{\mu}+2 \alpha^{\prime}(p \cdot \psi) \psi^{\mu}\right) e^{i p \cdot X}(x),
\end{aligned}
$$

\footnotetext{
${ }^{8}$ Estas amplitudes se obtuvieron para espaciotiempo plano, pero relacionaremos estos resultados con los de espacio curvo $A d S$, en los que estamos interesados, de la forma ya explicada en la Sección 3.2 .

${ }^{9} \mathrm{Si}$ consideráramos más de una $\mathrm{D} p$ - brana tendríamos un factor adicional que da la estructura no abeliana . En este caso, con una sola D $p$-brana, $N_{f}=1$, este factor no aparece.
} 

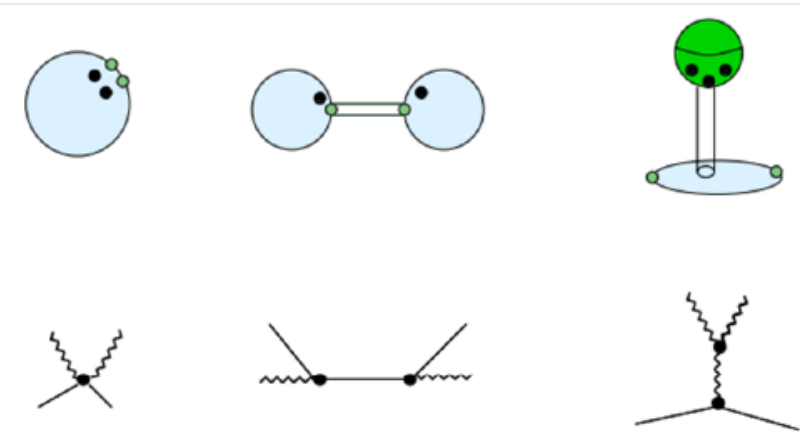

Figura 7.4: En este diagrama pueden verse los tres tipos de contribuciones presentes para la dispersión de dos cuerdas abiertas y dos cuerdas cerradas. Se muestran los diagramas de teoría de cuerdas en la parte superior y los correspondientes a la teoría de campos en la parte inferior. En los diagramas de cuerdas, los puntos oscuros corresponden a vértices de gravitones mientras que los claros corresponden a vértices de mesón escalar. La doble línea corresponde a un propagador de cuerda abierta en el segundo diagrama y a uno de cuerda cerrada en el tercero. En los diagramas de teoría de campos las líneas onduladas representan los gravitones mientras que las líneas continuas corresponden a los campos escalares. La primera contribución corresponde al término de contacto, la segunda corresponde al canal $s$ y el tercero al canal $t$ de intercambio de gravitones. Comparar con la Figura 7.5. Figura tomada de [86]

y para cuerdas cerradas son

$$
\begin{aligned}
V_{c}^{(-1,-1)}\left(z, \bar{z} ; h_{\mu \nu}, q\right)= & g_{c} e^{-\widetilde{\phi}(\bar{z})} e^{-\phi(z)} h_{\mu \nu} \widetilde{\psi}^{\mu}(\bar{z}) \psi^{\nu}(z) e^{i q \cdot X(\bar{z}, z)} \\
V_{c}^{(0,0)}\left(z, \bar{z} ; h_{\mu \nu}, q\right)= & -g_{c} \frac{2}{\alpha^{\prime}} h_{\mu \nu}\left(i \bar{\partial} X^{\mu}+\frac{\alpha^{\prime}}{2}(q \cdot \widetilde{\psi}) \widetilde{\psi}^{\mu}(\bar{z})\right)\left(i \partial X^{\nu}+\frac{\alpha^{\prime}}{2}(q \cdot \psi) \psi^{\nu}(z)\right) \\
& e^{i q \cdot X(\bar{z}, z)} .
\end{aligned}
$$

En nuestra notación $X^{\mu}$ y $\psi^{\mu}$ son los campos bosónicos y fermiónicos de la hoja de mundo respectivamente, mientras que $\phi$ y $\widetilde{\phi}$ son los fantasmas de Fadeev-Popov de la cuantización de la teoría de cuerdas. Las constantes de acoplamiento para cuerdas abiertas $g_{o}$ y cerradas $g_{c}$ son $g_{c} \equiv g_{s}$ y $g_{c}=g_{o}^{2}=g_{s}^{2}$.

Estamos interesados entonces en la amplitud de dispersión de dos cuerdas abiertas y dos cerradas. Esta está dada por la integral

$$
\begin{aligned}
& A_{\text {string }}\left(h_{1}, h_{4}, \epsilon_{2}, \epsilon_{3}\right)=\int_{\partial \mathbf{H}_{+}} d x \int_{\partial \mathbf{H}_{+}} d y \int_{z \in \mathbf{H}_{+}} d z d \bar{z} \int_{w \in \mathbf{H}_{+}} d w d \bar{w}\langle c(z) \widetilde{c}(\bar{z}) \times \\
& \left.V_{c}^{(0,0)}\left(z, \bar{z} ; h_{1 \mu \nu}, k_{1}\right) V_{c}^{(-1,-1)}\left(w, \bar{w} ; h_{4 \mu \nu}, k_{4}\right)(c(x)-c(y)) V_{o}^{(0)}\left(x, \epsilon_{2 \mu}, k_{2}\right) V_{o}^{(0)}\left(y, \epsilon_{3 \mu}, k_{3}\right)\right\rangle,
\end{aligned}
$$

donde utilizamos $V$ para notar el orden normal $: V$ : de los operadores de vértice, y $c$ y $\tilde{c}$ son fantasmas.

Quedan dos parámetros reales y dos complejos a determinar, asociados a las inserciones de los operadores de vértice de las cuerdas abiertas y cerradas respectivamente. La simetría $S L(2, R)$ de la hoja de mundo nos permite fijar tres parámetros reales, con lo que solo nos resta integrar sobre tres variables reales. Elegimos $x \rightarrow-\infty, y=1$ y $\operatorname{Re}(w)=0($ i.e. $\bar{w}=-w)$. 
Calculamos ahora el valor de expectación de vacío de (7.2.6),

$$
\langle f[\Phi]\rangle \equiv \int D \Phi e^{i S[\Phi]} f[\Phi],
$$

donde $\Phi \equiv\{X, \psi, \widetilde{\psi}, \phi, \widetilde{\phi}, c, \widetilde{c}\}$. La acción puede factorizarse como

$$
S[\Phi]=S[X, \psi, \widetilde{\psi}, \phi, \widetilde{\phi}, c, \widetilde{c}]=S_{X}[X]+S_{\psi}[\psi, \widetilde{\psi}]+S_{\phi}[\phi, \widetilde{\phi}]+S_{c}[c, \widetilde{c}] .
$$

Por lo tanto, cada integral de camino puede hacerse separadamente. Entonces, para calcular la amplitud de dispersión (7.2.6) podemos usar el valor de expectación de cada par de campos en el disco [85, 87, 86]

$$
\begin{aligned}
\left\langle X^{\mu}(z) X^{\nu}(w)\right\rangle & =-2 \alpha^{\prime} \eta^{\mu \nu} \ln (z-w), \\
\left\langle X^{\mu}(z) \widetilde{X}^{\nu}(\bar{w})\right\rangle & =-2 \alpha^{\prime} D^{\mu \nu} \ln (z-\bar{w}), \\
\left\langle\psi^{\mu}(z) \psi^{\nu}(w)\right\rangle & =\eta^{\mu \nu}(z-w)^{-1}, \\
\left\langle\psi^{\mu}(z) \widetilde{\psi}^{\nu}(\bar{w})\right\rangle & =D^{\mu \nu}(z-\bar{w})^{-1}, \\
\langle\phi(z) \phi(w)\rangle & =-\ln (z-w), \\
\langle\phi(z) \widetilde{\phi}(\bar{w})\rangle & =-\ln (z-\bar{w}), \\
\left\langle c\left(w_{1}\right) c\left(w_{2}\right) c(z)\right\rangle & =C_{\text {ghost }}\left(w_{1}-w_{2}\right)\left(w_{1}-z\right)\left(w_{2}-z\right), \\
\left\langle c\left(w_{1}\right) c\left(w_{2}\right) \widetilde{c}(\bar{z})\right\rangle & =C_{\text {ghost }}\left(w_{1}-w_{2}\right)\left(w_{1}-\bar{z}\right)\left(w_{2}-\bar{z}\right),
\end{aligned}
$$

donde $D^{\mu \nu}$ es una matriz diagonal con valor 1 en las direcciones paralelas a la $\mathrm{D} p$-brana de sabor y -1 en las direcciones perpendiculares. Con esto, el valor de expectación de (7.2.6) es

$$
\begin{aligned}
& \langle c(z) \widetilde{c}(\bar{z})(c(x)-c(y))\rangle\left\langle e^{-\widetilde{\phi}(\bar{z})} e^{-\phi(z)}\right\rangle g_{c}^{2} \frac{2}{\alpha^{\prime}} g_{o}^{2} \frac{1}{2 \alpha^{\prime}} h_{1 \mu \nu} h_{4 \rho \sigma} \epsilon_{2 \alpha} \epsilon_{3 \beta} \times \\
& \left\langle\widetilde{\psi^{\mu}}(\bar{z}) \psi^{\nu}(z) e^{i k_{1} \cdot X(\bar{z}, z)}\left(i \bar{\partial} X^{\rho}+\frac{\alpha^{\prime}}{2}\left(k_{4} \cdot \widetilde{\psi}\right) \widetilde{\psi}^{\rho}(\bar{w})\right)\left(i \partial X^{\sigma}+\frac{\alpha^{\prime}}{2}\left(k_{4} \cdot \psi\right) \psi^{\sigma}(w)\right) e^{i k_{4} \cdot X(\bar{w}, w)}\right. \\
& \left.\left(i \partial_{x} X^{\alpha}+2 \alpha^{\prime} k_{2} \cdot \psi \psi^{\alpha}\right) e^{i k_{2} \cdot X}(x)\left(i \partial_{y} X^{\beta}+2 \alpha^{\prime} k_{3} \cdot \psi \psi^{\beta}\right) e^{i k_{3} \cdot X}(y)\right\rangle .
\end{aligned}
$$

Deberíamos realizar ahora contracciones de Wick para 16 términos. Este cálculo se simplifica porque la contracción de dos campos en el mismo punto del disco se anula. Por otro lado, una mayor simplificación viene dada porque, en el caso de los mesones escalares, hay solamente un término no nulo. Esto se debe a que las "polarizaciones" de estos mesones en diez dimensiones, que corresponden a las direcciones perpendiculares a la $\mathrm{D} p$-brana, son por esto mismo perpendiculares a todos los momentos y polarizaciones de los demás campos.

En [86] Fotopoulos y Tseytlin habían encontrado un resultado en el régimen en que la teoría de supercuerdas se reduce a la de supergravedad. En aquel caso la integral es resuelta exclusivamente en la zona cercana a las singularidades, cálculos similares a los que realizaremos en la próxima Sección.

En [85. Stieberger obtuvo el resultado exacto, que puede separarse en dos términos como se anticipó en (7.2.1), donde el primer prefactor es

$$
\begin{aligned}
\mathcal{P}_{1}^{2 o 2 c, \text { scalar }=} & \int_{-\infty}^{+\infty} d x x^{2}(1+i x)^{u-1}(1-i x)^{u-1} \times \\
& \int_{C} d^{2} z(1-z)^{s}(1-\bar{z})^{s}(z+i x)^{\frac{t}{2}-1}(z-i x)^{\frac{t}{2}-1}(\bar{z}+i x)^{\frac{t}{2}-1}(\bar{z}-i x)^{\frac{t}{2}-1},
\end{aligned}
$$

y $\mathcal{K}_{1}^{202 c, s c a l a r}$ viene dado por la suma de las amplitudes de dispersión asociadas con los diagramas de Feynman que contribuyen a este proceso en el cálculo de supergravedad [86]. Como dijimos 
antes, escribimos solamente el primer término pues en el régimen cinemático en que estamos interesados este es el único relevante.

Si bien el prefactor necesario para construir la acción efectiva de dos cuerdas abiertas y dos cerradas viene dado formalmente por 7.2 .10 en el límite de $\tilde{t}$ pequeño y $\tilde{s}$ grande ${ }^{10}$, es muy complicado realizar este cálculo explícito. Daremos argumentos para sugerir que, para el caso de mesones escalares, este prefactor toma la misma forma que para el caso de glueballs estudiado en la Sección 7.1, es decir

$$
\frac{\pi \alpha^{\prime}}{4} \sum_{m=0}^{\infty} \delta\left(m-\frac{\alpha^{\prime} \tilde{s}}{4}\right)(m)^{\alpha^{\prime} \tilde{t} / 4},
$$

donde omitimos el término con dependencia $\frac{1}{\hat{t}}$ que indica que la contribución dominante es la del canal $t$, y nos enfocamos en la parte imaginaria que separa el intercambio de cuerdas excitadas [5]. En [47] se demostró que en este régimen de parámetros la dependencia $\tilde{s}$ da siempre un factor

$$
\left(\frac{\alpha^{\prime} \tilde{s}}{4}\right)^{\alpha^{\prime} \tilde{t} / 4}=\int_{0}^{\infty} d m \delta\left(m-\frac{\alpha^{\prime} \tilde{s}}{4}\right)(m)^{\alpha^{\prime} \tilde{t} / 4} \approx \sum_{m=1}^{\infty} \delta\left(m-\frac{\alpha^{\prime} \tilde{s}}{4}\right)(m)^{\alpha^{\prime} \tilde{t} / 4} .
$$

En el último paso tuvimos en cuenta que para $x \ll 1 / \sqrt{\lambda}$ la integral se parece a la suma. Para el régimen de $x$ pequeño el factor $(m)^{\alpha^{\prime} t \hat{t} / 4}$ es de orden uno 11 . Esta aproximación perderá validez para el régimen de $x$ exponencialmente pequeño (región $\mathbf{C}$ ). También aparece un factor que contiene el polo en $\tilde{t}$. De hecho, la expansión de OPE asociada a este proceso fue estudiada tanto para un par de supercuerdas cerradas [47] como para supercuerdas abiertas [88, 89], y en ambos casos resulta un factor de la forma

$$
\frac{\Gamma\left(-1-\frac{\alpha^{\prime} \tilde{t}}{4}\right)}{\Gamma\left(2+\frac{\alpha^{\prime} \tilde{t}}{4}\right)} e^{i \pi\left(1-\alpha^{\prime} t / 4\right)} \propto-\frac{1}{\alpha^{\prime} \tilde{t}}+O(1)
$$

donde en el último paso expandimos en $\alpha^{\prime} \tilde{t}$. Con esto corroboramos el hecho de que podemos despreciar otros términos que pudieran aparecer en $\mathcal{A}_{4}^{202 c, \text { scalar }}$ y $\mathcal{A}_{4}^{\text {2o2c,vector }}$, además del que no se anula para $\alpha^{\prime} \rightarrow 0$. Por otro lado, el análisis de OPE también separa el término donde la polarización de los gravitones y mesones incidentes ( $h$ y $\epsilon$ ) están contraídos en la combinación $\left(h_{1} h_{4}\right)\left(\epsilon_{2} \epsilon_{3}\right)$. Como veremos, esto está en perfecto acuerdo con nuestros cálculos.

Por último, esta suposición sobre el prefactor viene apoyada a posteriori por nuestros resultados. Veremos en las próximas Secciones que la parte cinemática del lagrangiano efectivo para el caso del mesón escalar es idéntica a la correspondiente a glueballs estudiada en la Sección 7.1 y en [5. Por otro lado, hemos visto que desde el punto de vista de la teoría de cuerdas la integral original a resolver de los operadores de vértices, que ulteriormente llevará a la amplitud total de teoría de cuerdas para genus cero, es la misma para vectores escalares y vectoriales. Esto se debe a que la única diferencia entre estos casos es la polarización de los mesones. Esto sugiere que es correcto utilizar el mismo prefactor estudiado aquí en el caso de los mesones vectoriales. 


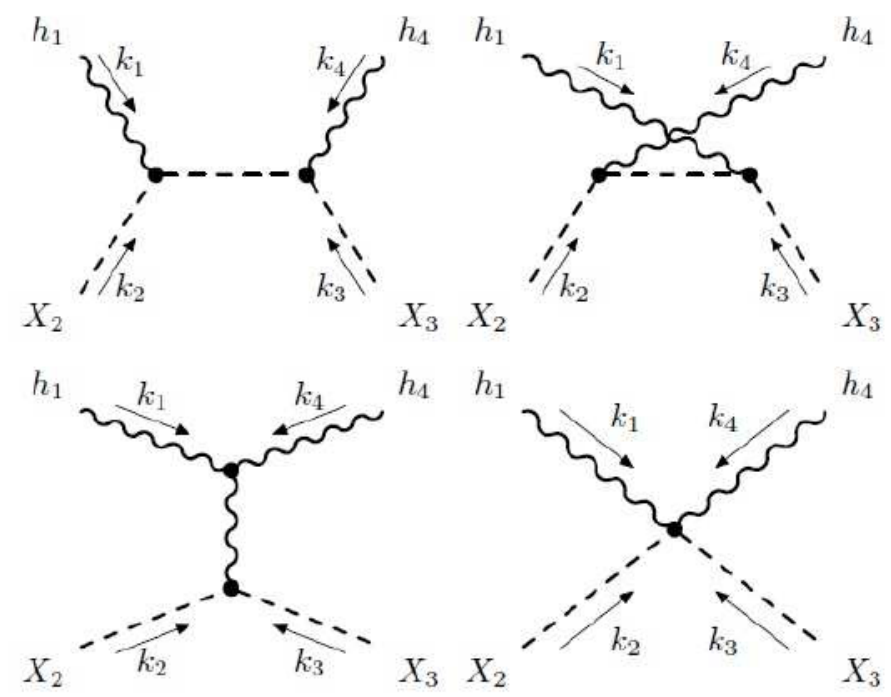

Figura 7.5: Los cuatro diagramas de Feynman correspondientes al cálculo holográfico de la amplitud de dispersión de cuatro puntos: canales $s, u, t$ e interacción de contacto. Las líneas onduladas representan a los gravitones $h_{1}, h_{4}$ así como los intermedios -duales holográficos de los fotones virtuales-, mientras que las líneas de trazos representan a las cuerdas abiertas $X_{2}, X_{3}$ e intermedios -duales holográficos de los mesones escalares-. Los momentos de los campos se representan con $k_{i}$.

\subsubsection{Amplitud de dispersión a partir de supergravedad}

Partimos de la acción 12

$$
S_{D B I}=-T_{7} \int d^{8} \xi \sqrt{-\operatorname{det}\left(\hat{P}[g]_{a b}+\left(2 \pi \alpha^{\prime}\right) F_{a b}\right)},
$$

y de la expansión de la métrica

$$
\sqrt{-g} \approx \sqrt{-g_{0}}\left[1+\frac{1}{2} H-\frac{1}{4} H_{b}^{a} H_{a}^{b}+\frac{1}{8} H^{2}+\frac{1}{6} H_{b}^{a} H_{c}^{b} H_{a}^{c}-\frac{1}{8} H H_{b}^{a} H_{a}^{b}+\frac{1}{48} H^{3}\right],
$$

donde $H \equiv H_{a}^{a}$ representa la traza. Hay ocho coordenadas paralelas a la D7-brana y dos perpendiculares, $x^{8}$ y $x^{9}$. Notamos los grados de libertad como $\xi^{a} \equiv X^{a}$ para los campos dentro de la D7-brana, $a=0, \ldots, 7$ y entendiendo a las coordenadas fuera de la D7-brana, $(8,9)$, como campos escalares $X^{I}$ con $I=1,2$, cuyos valores representan la fluctuación de la D7-brana.

Usando la parametrización estática e ignorando el campo vectorial $F_{a b}$, en el que no estamos interesados por el momento, identificamos los campos de nuestra teoría con las perturbaciones de la métrica asociadas con el gravitón y el campo escalar como

$$
\begin{array}{r}
H_{a b} \rightarrow \hat{g}_{a b} \equiv g_{a b}+2 g_{I(a} \partial_{b)} X^{I}+g_{I J} \partial_{a} X^{I} \partial_{b} X^{J}, \\
g_{a b}=\eta_{a b}+2 \kappa h_{a b}, g_{I J}=\delta_{I J}+2 \kappa h_{I J}, g_{a I}=2 \kappa h_{a I} .
\end{array}
$$

\footnotetext{
${ }^{10}$ Dado que las partículas son no masivas, $\tilde{s}+\tilde{t}+\tilde{u}=\sum_{i} m_{i}^{2}=0$, por lo que el valor absoluto de $\tilde{u} \approx-\tilde{s}$ también es grande.

${ }^{11}$ Ver nota al pie 4.

${ }^{12}$ En este Capítulo llamaremos $T_{7}$ a la tensión de la D7-brana en lugar de $\mu_{7}$ como en el Capítulo anterior.
} 
Nos concentraremos ahora en el caso en el que la polarización del gravitón es paralela a la D7-brana, es decir $h_{I J}=h_{a I}=0$. Expandiendo la $S_{D B I}$ obtenemos tres de los ingredientes necesarios para el cálculo de los diagramas de Feynman a nivel árbol. Estos serán análogos a los diagramas obtenidos en el límite de bajas energías de la teoría de cuerdas cerradas, al considerar las interacciones gravitón-dilatón en la Subsección 7.1.2. Estos son el término cinético asociado con los campos escalares y las interacciones del tipo $h X X$ y $h h X X$. Notemos que como el término escalar en $H$ es cuadrático, solo hace falta realizar la expansión hasta tercer orden. También tomaremos el vértice de tres gravitones, así como el propagador del gravitón, directamente de la acción de cuerdas cerradas [33, 84, 86, 13

El primer orden en la expansión 7.2 .16 da un término cinético $\frac{T_{7}}{2} \partial_{a} X \partial^{a} X{ }^{14}$. El propagador es entonces

$$
P_{I J}(k) \equiv\left\langle X_{I} X_{J}\right\rangle=-i \frac{\delta_{I J}}{T_{7} k^{2}} .
$$

El segundo y tercer órdenes en esta expansión producen los lagrangianos de interacción

$$
\begin{aligned}
L_{h X X} & =T_{7} \kappa\left[\frac{1}{2} h \partial^{a} X \partial_{a} X-h_{b}^{a} \partial^{b} X \partial_{a} X\right] \\
L_{h h X X} & =T_{7} \kappa^{2}\left[\frac{1}{4} h^{2} \partial^{a} X \partial_{a} X+2 h_{b}^{a} h_{c}^{b} \partial^{c} X \partial_{a} X-h h_{b}^{a} \partial^{b} X \partial_{a} X-\frac{1}{2} h_{b}^{a} h_{a}^{b} \partial^{c} X \partial_{c} X\right],
\end{aligned}
$$

donde $h=h_{a}^{a}$. Notemos que para el término de contacto $V_{h h X X}$ ignoraremos los términos que contengan al factor $h_{a}^{a}$ ya que los gravitones externos están en capa de masa (on-shell) y por ende tiene traza nula. Sin embargo, esto no es cierto para el vértice $V_{h X X}$ en el diagrama del canal $t$, pues aquí está involucrado un gravitón virtual que posee traza no nula. ${ }^{15}$ Llamando $k_{1}$ y $k_{2}$ a los momentos (entrantes) del campo escalar, obtenemos

$$
\begin{aligned}
V_{h X X}^{a b, I J} & =i T_{7} \kappa \delta^{I J}\left[\frac{1}{2} \eta^{a b}\left(k_{1} \cdot k_{2}\right)-\left(k_{1}^{a} k_{2}^{b}+k_{1}^{b} k_{2}^{a}\right)+\cdots\right] \\
V_{h h X X}^{a b, c d, I J}\left(k_{1}, k_{2}\right) & =4 i T_{7} \kappa^{2} \delta^{I J}\left[\eta^{b c}\left(k_{1}^{a} k_{2}^{d}+k_{1}^{d} k_{2}^{a}\right)-\frac{1}{2} \eta^{a c} \eta^{b d}\left(k_{1} \cdot k_{2}\right)\right]
\end{aligned}
$$

donde los puntos suspensivos indican términos irrelevantes.

Si calculamos la amplitud representada por el diagrama de contacto en la Figura 7.5, esta resulta

$$
\begin{aligned}
A_{c}\left(k_{1}, k_{2}, k_{3}, k_{4}\right) & =h_{1 a b} h_{4 d e} \delta_{I J} V_{h h X X}^{a b, d e, I J}\left(k_{2}, k_{3}\right) \\
& =i \kappa^{2} T_{7}\left[4\left(k_{2} h_{1} h_{4} k_{3}\right)+4\left(k_{3} h_{1} h_{4} k_{2}\right)-2\left(h_{1} h_{4}\right)\left(k_{2} \cdot k_{3}\right)\right],
\end{aligned}
$$

donde los índices de los factores entre paréntesis están completamente contraídos en el orden indicado.

Las amplitudes de los canales $s$ y $t$, luego de utilizar la transversalidad de las polarizaciones,

\footnotetext{
${ }^{13}$ Esto se debe a que los términos provenientes de $S_{D B I}$ son no dominantes.

${ }^{14}$ En adelante omitiremos los índices $I$ y $J$ cuando haya una suma sobre ellos con la delta de Kronecker $\delta_{I J}$.

${ }^{15}$ De hecho, para escribir el vértice de tres gravitones incluyendo un gravitón virtual deberíamos incluir en la expansión (7.2.16) un término $h_{I J}$ no nulo. Esto agrega un término extra que igualmente no contribuye, debido a la estructura del propagador del gravitón [86].
} 
la conservación de momento y la condición de partículas no masivas, resultar ${ }^{16}$

$$
\begin{aligned}
A_{s}\left(k_{1}, k_{2}, k_{3}, k_{4}\right) & =h_{1 a b} h_{4 c d} \delta_{I J} V_{h X X}^{a b, I K}\left(k_{2},-k_{1}-k_{2}\right) P_{K L}\left(-k_{1}-k_{2}\right) V^{c d, L J}\left(k_{1}+k_{2}, k_{3}\right) \\
& =-i \kappa^{2} T_{7} \frac{4}{s}\left(k_{2} h_{1} k_{2}\right)\left(k_{3} h_{4} k_{3}\right), \\
A_{u}\left(k_{1}, k_{2}, k_{3}, k_{4}\right) & =h_{1 a b} h_{4 c d} \delta_{I J} V_{h X X}^{a b, J K}\left(k_{3},-k_{1}-k_{3}\right) P_{K L}\left(-k_{1}-k_{3}\right) V^{c d, L I}\left(k_{1}+k_{3}, k_{2}\right) \\
& =-i \kappa^{2} T_{7} \frac{4}{u}\left(k_{3} h_{1} k_{3}\right)\left(k_{2} h_{4} k_{2}\right),
\end{aligned}
$$

donde $s=-2 k_{1} \cdot k_{2}=-2 k_{3} \cdot k_{4}$ y $u=-2 k_{1} \cdot k_{3}=-2 k_{2} \cdot k_{4}$.

Analicemos con un poco más de detalle la amplitud correspondiente al canal $t$, que será el más importante en el cálculo de la amplitud total. Necesitamos tres ingredientes: el vértice de tres gravitones derivado de $L_{h h h}$; el propagador del gravitón, dado por $L_{h h}^{\partial^{2}}$; y el vértice de un gravitón y dos escalares que ya hemos obtenido de $L_{h X X}$. El gravitón intercambiado, al ser virtual, tiene un término de traza en principio no nulo. Notemos también que como contraeremos el vértice $V_{h X X}$ con el propagador del gravitón, no es necesario simetrizar el vértice respecto de los índices $\alpha$ y $\beta$. Este debe ser contraído luego con el factor $S^{\rho \sigma, \gamma \delta, \alpha \beta}\left(k_{1}, k_{4}\right)$ obtenido en [84], que es la contracción del propagador del gravitón virtual con el vértice de tres gravitones. Luego resta contraer este factor con las polarizaciones de los gravitones externos $h_{1}$ y $h_{4}$, resultando

$$
\begin{aligned}
& h_{1 \rho \sigma} h_{4 \gamma \delta} S^{\rho \sigma, \gamma \delta, \alpha \beta}\left(k_{1}, k_{4}\right)= \\
& -\kappa\left[\frac{1}{2}\left(h_{1} h_{4}\right)^{\alpha \beta}+\frac{1}{2}\left(h_{1} h_{4}\right)^{\beta \alpha}+\frac{1}{t}\left(\left(k_{1} h_{4}\right)^{\alpha}\left(k_{4} h_{1}\right)^{\beta}+\left(k_{1} h_{4}\right)^{\beta}\left(k_{4} h_{1}\right)^{\alpha}\right)\right. \\
& -\frac{\left(h_{1} h_{4}\right)}{t}\left(k_{1}^{\alpha} k_{1}^{\beta}+k_{4}^{\alpha} k_{4}^{\beta}+\frac{1}{2} k_{4}^{\alpha} k_{1}^{\beta}+\frac{1}{2} k_{1}^{\alpha} k_{4}^{\beta}\right)-\frac{1}{t}\left(\left(k_{1} h_{4} k_{1}\right) h_{1}^{\alpha \beta}+\left(k_{4} h_{1} k_{4}\right) h_{4}^{\alpha \beta}\right) \\
& \left.+\frac{1}{t}\left(\left(k_{1} h_{4} h_{1}\right)^{\alpha} k_{1}^{\beta}+\left(k_{1} h_{4} h_{1}\right)^{\beta} k_{1}^{\alpha}+\left(k_{4} h_{1} h_{4}\right)^{\alpha} k_{4}^{\beta}+\left(k_{4} h_{1} h_{4}\right)^{\beta} k_{4}^{\alpha}\right)\right] .
\end{aligned}
$$

Tomando los índices correspondientes a la $\mathrm{D} p$-brana, $\alpha, \beta \rightarrow a, b$, y contrayendo este resultado con el vértice $V_{h X X}^{a b, I, J}$, obtenemos la amplitud para el proceso en el canal $t$

$$
\begin{aligned}
& A_{t}\left(k_{1}, k_{2}, k_{3}, k_{4}\right)=h_{1 c d} h_{4 e f} S^{c d, d f, a b}\left(k_{1}, k_{4}\right) V_{h X X}^{a b, I, J}\left(k_{2}, k_{3}\right) \delta_{I J}= \\
& \frac{-2 i \kappa^{2} T_{7}}{s u t}\left[\frac{1}{4}\left(h_{1} h_{4}\right)\left(t^{2}+u^{2}+s^{2}\right)+u\left[\left(k_{3} h_{1} h_{4} k_{1}\right)+\left(k_{4} h_{1} h_{4} k_{2}\right)\right]\right. \\
& +s\left[\left(k_{2} h_{1} h_{4} k_{1}\right)+\left(k_{4} h_{1} h_{4} k_{3}\right)\right]+t\left[\left(k_{2} h_{1} h_{4} k_{3}\right)+\left(k_{3} h_{1} h_{4} k_{2}\right)-\left(k_{4} h_{1} h_{4} k_{1}\right)\right]+ \\
& \left.2\left[\left(k_{4} h_{1} k_{2}\right)\left(k_{3} h_{4} k_{1}\right)+\left(k_{4} h_{1} k_{3}\right)\left(k_{2} h_{4} k_{1}\right)-\left(k_{4} h_{1} k_{4}\right)\left(k_{2} h_{4} k_{3}\right)-\left(k_{2} h_{1} k_{3}\right)\left(k_{1} h_{4} k_{1}\right)\right]\right],
\end{aligned}
$$

donde $t=-2 k_{1} \cdot k_{4}=-2 k_{2} \cdot k_{3}$. Notemos aquí que el factor $(s u)^{-1}$ fue extraído por conveniencia. En cambio, el factor $\frac{1}{t}$ representa realmente un polo en $t$.

Podemos construir entonces la amplitud completa a nivel árbol para este proceso, $A_{\text {total }}=$ $A_{c}+A_{s}+A_{u}+A_{t}$ correspondiente a la suma de las amplitudes de los cuatro diagramas de la Figura 7.5. Comparando esto con la Sección anterior vemos que esta estructura coincide con la amplitud de dispersión de teoría de cuerdas cerradas con dos gravitones y dos dilatones, a menos de un factor global su. Esto quiere decir que, dado que los diagramas de Feynman son los mismos, $A_{\text {total }}$ reproduce el factor cinemático de la amplitud de cuatro cuerdas cerradas obtenida desde teoría de cuerdas. De hecho, este factor es, como mostramos antes 7.1.6

$$
A_{4}^{\text {closed }}=-i \pi^{2} g_{c} \alpha^{\prime 3} \frac{\Gamma\left(-\frac{\alpha^{\prime} s}{4}\right) \Gamma\left(-\frac{\alpha^{\prime} u}{4}\right) \Gamma\left(-\frac{\alpha^{\prime} t}{4}\right)}{\Gamma\left(1+\frac{\alpha^{\prime} s}{4}\right) \Gamma\left(1+\frac{\alpha^{\prime} u}{4}\right) \Gamma\left(1+\frac{\alpha^{\prime} t}{4}\right)} \times K_{4}^{\text {closed }},
$$

\footnotetext{
${ }^{16}$ En el resto de esta Sección notaremos, por comodidad, $s, t$ y $u$ en lugar de $\tilde{s}, \tilde{t}$ y $\tilde{u}$ para la dispersión de cuerdas. Estas son las variables de Mandelstam en diez dimensiones.
} 


\subsubsection{Lagrangiano efectivo}

Obtendremos ahora la acción efectiva con interacciones de cuatro puntos que involucren dos gravitones y dos mesones escalares, partiendo de la amplitud de dispersión de teoría de cuerdas $A_{\text {total }}$ calculada en la Sección anterior. Recordemos que en régimen $0<\alpha^{\prime} t \ll 1 \alpha^{\prime} s \approx-\alpha^{\prime} u$. Por un lado habrá que hacer aproximaciones en el prefactor de la amplitud y por otro tenemos que encontrar los términos dominantes provenientes del factor cinemático, que producirán la amplitud de dispersión correspondiente al canal $t$.

Buscamos un lagrangiano de interacción de cuatro puntos. Para esto, comenzaremos escribiendo todos los términos que necesitaremos para reproducir la amplitud de dispersión del canal $t$. Escribimos cada término junto con su contribución a la amplitud de dispersión según las reglas de Feynman:

$$
\begin{aligned}
& \mathcal{L}_{A}= \partial_{n} h_{m p} \partial^{n} h^{m p} \partial_{M} X \partial^{M} X \rightarrow\left(h_{1} h_{4}\right) t^{2}, \\
& \mathcal{L}_{B}= \partial^{m} h^{p r} \partial^{n} h_{p r} \partial_{m} X \partial_{n} X \rightarrow \frac{1}{2}\left(h_{1} h_{4}\right)\left(s^{2}+u^{2}\right), \\
& \mathcal{L}_{C}=\partial_{m} h^{n p} \partial_{n} h_{p}^{m} \partial_{M} X \partial^{M} X \rightarrow-2 t\left(k_{4} k_{1} h_{4} k_{1}\right), \\
& \mathcal{L}_{D}=\partial^{m} h^{p r} \partial_{p} h_{r}^{n} \partial_{m} X \partial_{n} X \rightarrow-\frac{1}{2} s\left[\left(k_{4} h_{1} h_{4} k_{3}\right)+\left(k_{2} h_{1} h_{4} k_{1}\right)\right] \\
& \quad-\frac{1}{2} u\left[\left(k_{3} h_{1} h_{4} k_{1}\right)+\left(k_{4} h_{1} h_{4} k_{2}\right)\right], \\
& \mathcal{L}_{E}=\partial^{p} h^{m r} \partial_{p} h_{r}^{n} \partial_{m} X \partial_{n} X \rightarrow-t\left[\left(k_{2} h_{1} h_{4} k_{3}\right)+\left(k_{3} h_{1} h_{4} k_{2}\right)\right], \\
& \mathcal{L}_{F}=\partial_{p} h^{r m} \partial_{r} h^{p n} \partial_{m} X \partial_{n} X \rightarrow 2\left[\left(k_{2} h_{1} k_{4}\right)\left(k_{3} h_{4} k_{1}\right)+\left(k_{3} h_{1} k_{4}\right)\left(k_{2} h_{4} k_{1}\right)\right], \\
& \mathcal{L}_{G}=h^{r p} \partial_{r} \partial_{p} h^{m n} \partial_{m} X \partial_{n} X \rightarrow 2\left[\left(k_{1} h_{4} k_{1}\right)\left(k_{2} h_{1} k_{3}\right)+\left(k_{4} h_{1} k_{4}\right)\left(k_{2} h_{4} k_{3}\right)\right] .
\end{aligned}
$$

Comparando estas amplitudes parciales con la amplitud del polo del gravitón en el canal $t$, resulta un lagrangiano efectivo de la forma

$$
\mathcal{L}_{\text {eff }} \propto \frac{1}{4} \times \mathcal{L}_{A}+\frac{1}{2} \times \mathcal{L}_{B}+\frac{1}{2} \times \mathcal{L}_{C}-2 \times \mathcal{L}_{D}-1 \times \mathcal{L}_{E}+1 \times \mathcal{L}_{F}-1 \times \mathcal{L}_{G}
$$

Falta aquí un factor global su que, por conveniencia, será incluido en el prefactor.

Considerando la perturbación de la métrica 6.1.11 $h^{a b} \equiv \frac{1}{2}\left(A^{a} v^{b}+A^{b} v^{a}\right)$, podemos reescribir los términos del lagrangiano que contienen gravitones como

$$
\begin{aligned}
\partial_{b} h_{a c} \partial^{b} h^{a c} & =\frac{1}{2} v^{c} v_{c} \partial_{b} A_{a} \partial^{b} A^{a}, \partial^{a} h^{c d} \partial^{b} h_{c d}=\frac{1}{2} v^{c} v_{c} \partial^{a} A_{d} \partial^{b} A^{d}, \\
\partial_{c} h^{d a} \partial_{d} h^{c b} & =\frac{1}{4} v^{a} v^{b} \partial_{c} A^{d} \partial_{d} A^{c}, \partial_{a} h^{b c} \partial_{b} h_{c}^{a}=\frac{1}{4} v^{c} v_{c} \partial_{a} A^{b} \partial_{b} A^{a}, \\
\partial^{a} h^{c d} \partial_{c} h_{d}^{b} & =\frac{1}{4} v^{d} v_{d} \partial^{a} A^{c} \partial_{c} A^{b}, \partial^{c} h^{a d} \partial_{c} h_{d}^{b}=\frac{1}{4}\left(v^{d} v_{d} \partial^{c} A^{a} \partial_{c} A^{b}+v^{a} v^{b} \partial^{c} A^{d} \partial_{c} A_{d}\right) .
\end{aligned}
$$

Con esta elección de polarización para los gravitones el término $\mathcal{L}_{G}$ se anula. Considerando que los términos proporcionales a $t$ en la amplitud de dispersión serán de orden subdominante en este régimen, podemos quedarnos solamente con los términos relevantes mediante el lagrangiano efectivo invariante de gauge $\left.\right|^{17}$

$$
\mathcal{L}_{e f f} \propto \frac{1}{4}\left[2 v^{i} v_{i} \partial_{m} X \partial^{p} X F^{m n} F_{p n}+v^{i} v^{j} \partial_{i} X \partial_{j} X F^{m n} F_{m n}\right]
$$

donde definimos $F_{m n}=\partial_{m} A_{n}-\partial_{n} A_{m}$. El término más importante de este lagrangiano es el primero, pues da lugar a términos de la forma $\frac{s^{2}}{t}$ y $\frac{s}{t}$ en la amplitud. El diagrama de Feynman correspondiente a este diagrama efectivo se muestra en la Figura 7.6.

\footnotetext{
${ }^{17}$ Consideramos transformaciones del campo de gauge $A^{m}$.
} 


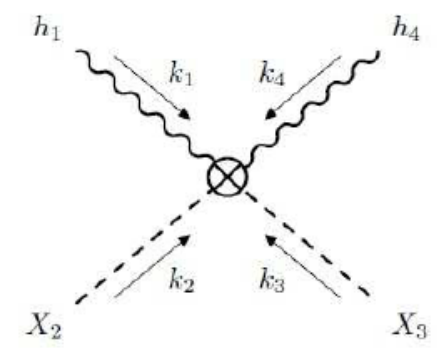

Figura 7.6: Interacción efectiva de cuatro puntos. Las líneas onduladas representan gravitones físicos y las líneas de trazos cuerdas abiertas -duales holográficos de los mesones escalares-.

\subsubsection{Cálculo del tensor hadrónico}

Siguiendo nuestreo paper [3], utilizaremos la misma aproximación realizada en la Sección 7.1 correspondiente al trabajo [5], por la cual construimos una acción efectiva para espaciotiempo curvo partiendo de (7.2.31) pero contrayendo los índices correspondientes con la métrica en diez dimensiones y agregando la raíz del determinante de la métrica. Es decir 18

$$
n_{\mu}^{*} n_{\nu} I m_{e x c} T^{\mu \nu}=\frac{\pi \alpha^{\prime}}{8} \sum_{m=1}^{\infty} \int d \Omega_{3} d r \sqrt{-g} v_{i} v^{i} \partial^{k} X^{*}(P) \partial^{n} X(P) F_{k p}^{*}(q) F_{n}^{p}(q) \delta\left(m-\frac{\alpha^{\prime} \tilde{s}}{4}\right) .
$$

Omitimos aquí las integrales en el espaciotiempo de Minkowski $\left(x_{0}, \cdots, x_{3}\right)$ pues estas solo aportan una delta de conservación del momento, que ya fue aplicada. Las soluciones a las ecuaciones de movimiento para los campos de interés son (ver [1] y Capítulo 6)

$$
\begin{aligned}
A_{\mu}(q) & =n_{\mu} f(r) e^{i q \cdot x}, \\
A_{r}(q) & =\frac{-i q \cdot n}{q^{2}} f^{\prime}(r) e^{i q \cdot x}, \\
X^{l} & =\frac{c_{i}^{l}}{\Lambda R^{3}}\left(\frac{r}{\Lambda R^{2}}\right)^{-\Delta} Y^{l}\left(\Omega_{3}\right) e^{i P \cdot x},
\end{aligned}
$$

donde $\Delta$ es la dimensión de escaleo de los mesones y $c_{i}$ es una constante adimensional. Realizando el cambio de variables $\omega=\frac{q R^{2}}{r}$, escribimos explícitamente $f(r)=\omega K_{1}(\omega)$, donde $K_{1}$ es la función de Bessel modificada de segunda especie. El tensor de campo resulta

$$
\begin{aligned}
& F_{\mu \nu}=i\left(q_{\mu} A_{\nu}-q_{\nu} A_{\mu}\right)=i\left(q_{\mu} n_{\nu}-q_{\nu} n_{\mu}\right) f(r) e^{i q \cdot x} \\
& F_{\mu r}=i\left(q_{\mu}-n_{\mu} \frac{q^{2}}{q \cdot n}\right) A_{r}=\left(\frac{q \cdot n}{q^{2}} q_{\mu}-n_{\mu}\right) f^{\prime}(r) e^{i q \cdot x}
\end{aligned}
$$

y la derivada del campo escalar es ${ }^{19}$

$$
\partial_{\nu} X=i P_{\nu} X, \quad \partial_{r} X=\frac{-\Delta}{r} X,
$$

\footnotetext{
${ }^{18}$ Como dijimos en la Sección 7.1, en la región $\mathbf{B}$ tomamos a la cantidad $\tilde{s}$ como un escalar. En la región C esta será un operador diferencial (ver Subsección 7.5 y nuestro trabajo [3].

${ }^{19} \mathrm{~A}$ partir de aquí omitimos el índice $l$ que identifica el mesón escalar $X$ en la torre de estados de Kaluza-Klein.
} 
de donde construimos los diferentes términos del lagrangiano efectivo. Para el campo de gauge

$$
\begin{aligned}
F_{r p}^{*} F_{r}^{p}= & \frac{R^{2}}{r^{2}} \eta^{\rho \sigma} F_{r \rho}^{*} F_{r \sigma}=\frac{R^{2}}{r^{2}}\left(f^{\prime}\right)^{2}\left[\left(n \cdot n^{*}\right)-\frac{(q \cdot n)\left(q \cdot n^{*}\right)}{q^{2}}\right] \\
F_{r p}^{*} F_{\mu}^{p}= & \frac{R^{2}}{r^{2}} \eta^{\rho \sigma} F_{r \rho}^{*} F_{\mu \sigma}=i \frac{R^{2}}{r^{2}} f f^{\prime} q_{\mu}\left[\left(n \cdot n^{*}\right)-\frac{(q \cdot n)\left(q \cdot n^{*}\right)}{q^{2}}\right], \\
F_{\mu p}^{*} F_{\nu}^{p}= & \frac{R^{2}}{r^{2}} \eta^{\rho \sigma} F_{\mu \rho}^{*} F_{\nu \sigma}+\frac{r^{2}}{R^{2}} F_{\mu r}^{*} F_{\nu r} \\
= & \frac{R^{2}}{r^{2}} f^{2}\left[q_{\mu} q_{\nu}\left(n^{*} \cdot n\right)+n_{\mu}^{*} n_{\nu} q^{2}-q_{\mu} n_{\nu}\left(q \cdot n^{*}\right)-q_{\nu} n_{\mu}^{*}(q \cdot n)\right] \\
& +\frac{r^{2}}{R^{2}}\left(f^{\prime}\right)^{2}\left[n_{\mu}^{*} n_{\nu}+q_{\mu} q_{\nu} \frac{(q \cdot n)\left(q \cdot n^{*}\right)}{q^{4}}-q_{\mu} n_{\nu} \frac{\left(q \cdot n^{*}\right)}{q^{2}}-q_{\nu} n_{\mu}^{*} \frac{(q \cdot n)}{q^{2}}\right] \\
= & \left(n_{\mu}^{*}-q_{\mu} \frac{q \cdot n^{*}}{q^{2}}\right)\left(n_{\nu}-q_{\nu} \frac{q \cdot n}{q^{2}}\right) \frac{\omega^{4}}{R^{2}}\left[K_{0}^{2}(\omega)+K_{1}^{2}(\omega)\right] \\
& +q_{\mu} q_{\nu}\left(n^{*} \cdot n-\frac{(q \cdot n)\left(q \cdot n^{*}\right)}{q^{2}}\right) \frac{\omega^{4}}{q^{2} R^{2}} K_{1}^{2}(\omega),
\end{aligned}
$$

y para el campo escalar

$$
\begin{aligned}
\partial^{r} X^{*}(P) \partial^{r} X(P) & =\frac{r^{2} \Delta^{2}}{R^{4}}|X|^{2}, \quad \partial^{\mu} X^{*}(P) \partial^{r} X(P)=-\frac{i \Delta}{r} P^{\mu}|X|^{2}, \\
\partial^{\mu} X^{*}(P) \partial^{\nu} X(P) & =\frac{R^{4}}{r^{4}} P^{\mu} P^{\nu}|X|^{2} .
\end{aligned}
$$

Como extraeremos de aquí $T^{\mu \nu}$, todos los términos de la forma $q \cdot n$ y $q \cdot n^{*}$ resultarán en términos proporcionales a $q^{\nu}$ y $q^{\mu}$ respectivamente, por lo que se anularán luego lo contraeremos con el tensor leptónico, como se explicó en el Capítulo 4. Nos resta expandir el lagrangiano efectivo en términos de las soluciones de los campos. Podemos reescribir el lagrangiano efectivo (7.2.31) como

$$
\mathcal{L}_{4}^{e f f}=\mathcal{L}_{4, A}^{e f f}+\mathcal{L}_{4, B}^{e f f}+\mathcal{L}_{4, C}^{e f f}+\mathcal{L}_{4, D}^{e f f}
$$

con

$$
\begin{aligned}
\mathcal{L}_{4, A}^{e f f}= & \partial^{r} X^{*}(P) \partial^{r} X(P) F_{r p}^{*} F_{r}^{p}=\frac{\Delta^{2}}{R^{2}}\left(f^{\prime}\right)^{2}\left(n \cdot n^{*}\right)|X|^{2}, \\
\mathcal{L}_{4, B}^{e f f}= & \partial^{\mu} X^{*}(P) \partial^{r} X(P) F_{\mu p}^{*} F_{r}^{p}=\frac{R^{2} \Delta}{r^{3}} f f^{\prime}\left(n \cdot n^{*}\right)|X|^{2}(P \cdot q)=\mathcal{L}_{4, C}^{e f f}, \\
\mathcal{L}_{4, D}^{e f f}= & \partial^{\mu} X^{*}(P) \partial^{\nu} X(P) F_{\mu p}^{*} F_{\nu}^{p}=\frac{R^{4}|X|^{2}}{r^{4}} \times\left[\frac{R^{2}}{r^{2}}\left(n \cdot n^{*}\right) f^{2}(P \cdot q)^{2}+\right. \\
& \left.\left(\frac{R^{2}}{r^{2}} q^{2} f^{2}+\frac{r^{2}}{R^{2}}\left(f^{\prime}\right)^{2}\right)(P \cdot n)\left(P \cdot n^{*}\right)\right] .
\end{aligned}
$$

Notemos aquí que los términos $\mathcal{L}_{4, A}^{\text {eff }}, \mathcal{L}_{4, B}^{\text {eff }}$ y $\mathcal{L}_{4, C}^{\text {eff }}$ son subdominantes en comparación con el término que multiplica $\left(n \cdot n^{*}\right)$ en $\mathcal{L}_{4, D}^{e f f}$. Este término contiene un factor $(P \cdot q)^{2} \propto s^{2}$, por lo que podemos despreciar la contribución proveniente de $\mathcal{L}_{4, A}^{\text {eff }}, \mathcal{L}_{4, B}^{\text {eff }}$ y $\mathcal{L}_{4, C}^{\text {eff }} \cdot{ }^{20}$ Para obtener ahora la

\footnotetext{
${ }^{20}$ Esto es muy similar a lo que ocurría en el caso de dispersión de glueballs en la Subsección 7.1.4.
} 
parte imaginaria de $T^{\mu \nu}$ resta extraer las polarizaciones $n$ y $n^{*}$ según $(7.2 .32)$, resultando

$$
\begin{aligned}
\operatorname{Im}_{e x c} T^{\mu \nu}= & \frac{\pi \alpha^{\prime}}{8} \sum_{m=1}^{\infty} \int d \Omega_{3} d r \sqrt{-g_{a b}} v^{i} v_{i} \delta\left(m-\frac{\alpha^{\prime} \tilde{s}}{4}\right) \times \\
& \left\{P^{\mu} P^{\nu} \times\left[\frac{R^{4}|X|^{2}}{r^{4}}\left(\frac{R^{2}}{r^{2}} q^{2} f^{2}+\frac{r^{2}}{R^{2}}\left(f^{\prime}\right)^{2}\right)\right]+\eta^{\mu \nu} \times\left[\frac{R^{6}|X|^{2}}{r^{6}} f^{2}(P \cdot q)^{2}\right]\right\} .
\end{aligned}
$$

Los factores proporcionales a los tensores $\eta^{\mu \nu}$ y $P^{\mu} P^{\nu}$ contribuyen a las funciones de estructura $F_{1}$ and $F_{2}$ respectivamente

\section{Nuevas relaciones entre $F_{1}$ y $F_{2}$ para $x$ pequeño (Región B)}

Nuestro objetivo en esta Sección es el de calcular las funciones de estructura $F_{1}$ y $F_{2}$. Esto lo haremos por simple comparación de (7.2.46) con la descomposición de Lorentz del tensor hadrónico para partículas con espín cero 4.3.2). Recordemos que utilizamos la expresión (7.2.32) para la parte imaginaria de $T_{\mu \nu}$, la que debe integrarse en las coordenadas radial $r$ y angulares $\Omega$ en $S^{3}$. Utilizando la condición de normalización para los armónicos esféricos en $S^{3}{ }^{21}$

$$
\int d \Omega_{3} \sqrt{\tilde{g}} v_{i} v^{i} Y\left(\Omega_{3}\right) Y^{*}\left(\Omega_{3}\right)=\rho_{3} R^{2},
$$

así como la definición de las integrales

$$
I_{j, n}=\int_{0}^{\infty} d \omega \omega^{n} K_{j}^{2}(\omega),
$$

donde $\omega=\frac{q R^{2}}{r}$. Definimos también

$$
\begin{aligned}
& I_{1}=\frac{\pi \alpha^{\prime}}{8} \sum_{m=1}^{\infty} R^{6} q^{2} \int d \Omega_{3} d r \sqrt{g_{8}} v_{i} v^{i}|X|^{2} \frac{f^{2}(r)}{r^{6}} \delta\left(m-\frac{\alpha^{\prime} s R^{2}}{4 r^{2}}\right), \\
& I_{0}=\frac{\pi \alpha^{\prime}}{8} \sum_{m=1}^{\infty} R^{2} \int d \Omega_{3} d r \sqrt{g_{8}} v_{i} v^{i}|X|^{2} \frac{\left(f^{\prime}(r)\right)^{2}}{r^{2}} \delta\left(m-\frac{\alpha^{\prime} s R^{2}}{4 r^{2}}\right) .
\end{aligned}
$$

Para estudiar $I_{1}$ definimos $r_{0}=\Lambda R^{2}, r_{m}^{2}=\frac{\alpha^{\prime} s R^{2}}{4 m}, f(r(\omega))=\omega K_{1}(\omega)$ y $f^{\prime}(r)=\partial_{r} f(r)=$ $\frac{\omega^{3}}{q R^{2}} K_{0}(\omega)$, y utilizamos 7.2.48. Para $r_{m}$ y $\omega$ podemos reemplazar la suma por una integral en $m$, y luego integrar en $\omega$, que resulta $\omega_{m}=\frac{q R^{2}}{r_{m}}$. Considerando el jacobiano

$$
d m=\frac{\omega}{2 x \sqrt{4 \pi g_{c} N}} d \omega,
$$

\footnotetext{
${ }^{21}$ En el caso general de una $S^{p-4}$ esfera, correspondiente al fondo con $\mathrm{D} p$ branas de sabor, que se tratará en la Sección 7.4 , tendremos la expresión análoga

$$
\int d \Omega_{p-4} \sqrt{\tilde{g}} v_{i} v^{i} Y\left(\Omega_{p-4}\right) Y^{*}\left(\Omega_{p-4}\right)=\rho_{p} R^{2} .
$$


resulta un valor explícito para $I_{1}$, a saber

$$
\begin{aligned}
I_{1} & =\frac{\pi \alpha^{\prime}}{8 \Lambda^{2}}\left|c_{i}\right|^{2} \rho_{3} R^{5} \sum_{m=1}^{\infty} \int d r\left(\frac{r}{R}\right)^{3}\left(\frac{r}{r_{0}}\right)^{-2 \Delta} \frac{f^{2}(r)}{r^{6}} \frac{\delta\left(r-r_{m}\right)}{\left|\frac{\alpha^{\prime} s R^{2}}{2 r^{3}}\right|} \\
& =\rho_{3}\left|c_{i}\right|^{2} \frac{\pi}{4 s \Lambda^{2}}\left(\frac{\Lambda^{2}}{q^{2}}\right)^{\Delta} \sum_{m=1}^{\infty} \omega^{2 \Delta+2} K_{1}^{2}(\omega) \\
& \approx \rho_{3}\left|c_{i}\right|^{2} \frac{\pi}{4 s \Lambda^{2}}\left(\frac{\Lambda^{2}}{q^{2}}\right)^{\Delta} \int_{1}^{\infty} \frac{d \omega}{2 x \sqrt{4 \pi g_{c} N}} \omega^{2 \Delta+3} K_{1}^{2}(\omega) \\
& =\rho_{3}\left|c_{i}\right|^{2} \frac{\pi}{4 s q^{2}}\left(\frac{\Lambda^{2}}{q^{2}}\right)^{\Delta-1} \frac{\Lambda^{2}}{2 x \sqrt{4 \pi g_{c} N}} I_{1,2 \Delta+3} .
\end{aligned}
$$

La otra integral, $I_{2}$, se calcula de la misma manera. Teniendo en cuenta que $s=-(P+q)^{2}=$ $q^{2}\left(-1+\frac{1}{x}-t\right) \approx \frac{q^{2}}{x}$ para este régimen, obtenemos

$$
\begin{aligned}
& I_{1}=\rho_{3}\left|c_{i}\right|^{2} \frac{\pi}{8}\left(\frac{\Lambda^{2}}{q^{2}}\right)^{\Delta-1} \frac{1}{\sqrt{4 \pi g_{c} N}} I_{1,2 \Delta+3}, \\
& I_{0}=\rho_{3}\left|c_{i}\right|^{2} \frac{\pi}{8}\left(\frac{\Lambda^{2}}{q^{2}}\right)^{\Delta-1} \frac{1}{\sqrt{4 \pi g_{c} N}} I_{0,2 \Delta+3} .
\end{aligned}
$$

Al comparar entonces nuestro tensor hadrónico con su descomposición en funciones de estructura, resulta

$$
\begin{aligned}
& F_{1}=\frac{\pi^{2}}{16 x^{2}} \rho_{3}\left|c_{i}\right|^{2}\left(\frac{\Lambda^{2}}{q^{2}}\right)^{\Delta-1} \frac{1}{\sqrt{4 \pi g_{c} N}} I_{1,2 \Delta+3} \\
& F_{2}=\frac{\pi^{2}}{8 x} \rho_{3}\left|c_{i}\right|^{2}\left(\frac{\Lambda^{2}}{q^{2}}\right)^{\Delta-1} \frac{1}{\sqrt{4 \pi g_{c} N}}\left(I_{0,2 \Delta+3}+I_{1,2 \Delta+3}\right) .
\end{aligned}
$$

Observamos aquí un comportamiento de la forma $F_{1} \propto \frac{1}{x^{2}} I_{1}$ y $F_{2} \propto \frac{1}{x}\left(I_{1}+I_{0}\right)$. De estas funciones extraemos inmediatamente la relación tipo Callan-Gross

$$
F_{2}=2 x F_{1}\left(1+\frac{I_{0}}{I_{1}}\right)=2 x F_{1} \frac{2 \Delta+3}{\Delta+2},
$$

donde hemos utilizado la identidad $I_{1, n}=\frac{n+1}{n-1} I_{0, n}$, que puede demostrarse a partir de la definición de estas funciones. La relación de Callan-Gross usual para QCD es $F_{2}=2 x F_{1}$ [63, 64, 65, 66] , mientras que aquí aparece un factor nuevo, idénticamente a lo ocurrido en [5] y expuesto en la Subsección 7.1.4 de este trabajo. Este factor depende exclusivamente de las dimensiones de escaleo $\Delta=l+2$ para los mesones escalares. Estos nuevos resultados fueron publicados en [3].

\subsection{DIS desde mesones vectoriales}

Queremos ahora construir el tensor hadrónico para mesones vectoriales en el modelo de D3D7-branas. Para esto tenemos que considerar la amplitud de dispersión de cuatro puntos con dos cuerdas abiertas y dos cerradas $\mathcal{A}_{4}^{202 c, v e c t o r}$. Las cuerdas que interactúan son de las mismas especies que en la Sección anterior: dos gravitones con una polarización (6.1.11) tal que representarán fotones virtuales en 5 dimensiones, y dos mesones vectoriales en la $\mathrm{D} p$-brana de sabor. La diferencia radicará en que aquí las polarizaciones de los mesones serán paralelas a la $\mathrm{D} p$ brana, lo que hará los cálculos algo más engorrosos. Comenzaremos con una polarización 
general para los mesones, de la que puede extraerse como un caso particular el de mesones escalares estudiado en la Sección anterior, y luego particularizaremos para mesones vectoriales definidos sobre la $\mathrm{D} p$-brana.

Este cálculo, si bien se hace en esta Sección para el caso particular de D3D7-branas, veremos que será válido para modelos provenientes de teoría de cuerdas tipo IIA y IIB y para distintas dimensiones de las Dp-branas de sabor. Esto se estudiará en la Sección 7.4, de cuyos resultados pueden derivarse fácilmente las expresiones para los modelos de $\mathrm{D} 4 \mathrm{D} 6 \overline{\mathrm{D} 6}$ y D4D8 $\overline{\mathrm{D} 8}$-branas.

La exposición será similar a la de la Sección anterior, aunque sin detenernos para volver a deducir los resultados ya obtenidos allí. En la Subsección 7.3.1 haremos unos comentarios sobre la amplitud de dispersión desde teoría de cuerdas y justificaremos el cálculo desde supergravedad. En la Subsección 7.3.2 realizaremos este cálculo de supergravedad, y finalmente en la Subsección 7.3.4 calcularemos todas las funciones de estructura para el caso vectorial.

\subsubsection{Amplitud de dispersión de cuatro puntos para cuerdas abiertas y cerradas}

Sea la amplitud de cuatro puntos para dos cuerdas abiertas y dos cerradas $\mathcal{A}_{4}^{202 c, v e c t o r}$. Estamos interesados en la contribución dominante en la expansión en genus, es decir, la correspondiente al límite planar de la teoría de gauge. Podemos expandir $\mathcal{A}_{4}^{202 c, v e c t o r}$ como una suma de contribuciones [85]

$$
\mathcal{A}_{4}^{202 c, \text { vector }}=\mathcal{P}_{1}^{202 c, \text { vector }} \mathcal{K}_{1}^{202 c, \text { vector }}+\mathcal{P}_{2}^{202 c, \text { vector }} \mathcal{K}_{2}^{202 c, \text { vector }}+\ldots
$$

Podemos obtener un lagrangiano de interacción efectivo $\mathcal{L}_{h h B B}^{\text {eff }}$ de cuatro puntos de la misma manera que en la Sección anterior, evaluando $\left.\mathcal{K}_{1}^{202 c, v e c t o r}\right|_{\tilde{t}=0}$. Este lagrangiano efectivo representará ahora la interacción de dos gravitones con la misma polarización (6.1.11) que en el caso anterior, con dos mesones vectoriales holográficos $B_{a}$, conservando la nomenclatura del Capítulo 6.

Procederemos siguiendo el método ii) descripto en la Sección anterior. Este consiste en considerar el límite de bajas energías de la acción de la teoría de cuerdas, que contiene cuerdas abiertas adheridas a las $\mathrm{D} p$-branas así como cuerdas cerradas en el interior del espacio, y en este contexto considerar el límite de las interacciones entre cuerdas abiertas y cerradas en el límite $\alpha^{\prime} \rightarrow 0$. Esto nos dará una acción de supergravedad en diez dimensiones $S_{S U G R A}$ que contendrá gravitones (así como dilatones y tensor antisimétrico), y la acción de Dirac-BornInfeld $S_{D B I}$, que es la acción de bajas energías en el volumen de mundo de la $\mathrm{D} p$-brana de sabor.

El propagador del gravitón y el vértice de tres gravitones se obtienen de $S_{S U G R A}$ mientras que el propagador del mesón holográfico $B_{a}$ y los términos de interacción de cuatro puntos $L_{h h B B}$ y de tres puntos $L_{h B B}$ saldrán de $S_{D B I}$. Con esto podemos construir todos los diagramas de Feynman que contribuyen a orden árbol. Estos son, al igual que en el caso de la Sección anterior, el término de contacto de cuatro puntos y los canales $s, t$ y $u$. El polo del gravitón del canal $t$ dará la parte cinemática del lagrangiano efectivo $\mathcal{L}_{h h B B}^{\text {eff }}$. Tengamos en cuenta que hay que tomar dos límites distintos, a saber $\alpha^{\prime} \rightarrow 0$ y luego $\tilde{t} \rightarrow 0$, que tras agregar el prefactor compondrá $\mathcal{L}_{h h B B}^{e f f}$. Este prefactor ha sido derivado en [85] en términos de integrales sobre la hoja de mundo, y supondremos que será similar al prefactor obtenido para mesones escalares, dada la similaridad de sus amplitudes desde teoría de cuerdas. Bajo esta suposición y obtuvimos, en [3] y la Sección 7.2 de esta Tesis, resultados consistentes con los obtenidos para glueballs ([5] y Sección 7.1. 


\subsubsection{Amplitud de dispersión a partir de supergravedad}

Partimos de la acción de Dirac-Born-Infeld para una sola D7-brana como hicimos en la Subsección 7.2 .2 ,

$$
S_{D B I}=-T_{7} \int d^{8} \xi \sqrt{-\operatorname{det}\left(\hat{P}[g]_{a b}+\left(2 \pi \alpha^{\prime}\right) F_{a b}\right)} .
$$

Expandimos nuevamente la raíz del determinante de la métrica al igual que en 7.2 .15

$$
\sqrt{-g} \approx \sqrt{-g_{0}}\left[1+\frac{1}{2} H-\frac{1}{4} H_{b}^{a} H_{a}^{b}+\frac{1}{8} H^{2}+\frac{1}{6} H_{b}^{a} H_{c}^{b} H_{a}^{c}-\frac{1}{8} H H_{b}^{a} H_{a}^{b}+\frac{1}{48} H^{3}\right],
$$

donde $H \equiv H_{a}^{a}$ es una traza y $g_{0}$ la métrica del fondo sin perturbar. En este caso es necesario el siguiente orden en la expansión anterior,. Este es

$$
\sqrt{-g_{0}}\left[\frac{1}{384} H^{4}+\frac{1}{32} H_{b}^{a} H_{a}^{b}\left(H_{d}^{c} H_{c}^{d}-H^{2}\right)+\frac{1}{12} H H_{b}^{a} H_{c}^{b} H_{a}^{c}-\frac{1}{8} H_{b}^{a} H_{c}^{b} H_{d}^{c} H_{a}^{d}\right] .
$$

Estos términos de interacción cuártica nos permiten obtener un término de interacción de cuatro puntos en el lagrangiano de bajas energías. Para esto, consideramos el caso de métrica plana $\left(g_{0}\right)_{a b}=\eta_{a b}$ y gauge estático. Elegimos las polarizaciones de los mesones paralelas a las D7 branas y resulta

$$
H_{a b} \rightarrow 2 \kappa h_{a b}+\left(2 \pi \alpha^{\prime}\right) G_{a b},
$$

donde $G_{a b}=\partial_{a} B_{b}-\partial_{b} B_{a}$ el $U(1)$ es el tensor de campo defindo en la D7-brana 22

Podemos extraer el término del lagrangiano asociado a la interacción cuártica $h h B B$, que se muestra en la Figura 7.7 con el resto de los diagramas, que es

$$
L_{h h B B}=T_{7} \kappa^{2}\left[\frac{1}{8} h^{2} G^{a b} G_{a b}+2 h h^{a b} G_{b c} G_{a}^{c}-\frac{1}{4} h^{a b} h_{a b} G^{c d} G_{c d}-h^{a b} h^{c d} G_{b c} G_{d a}-2 h^{a b} h_{b c} G^{c d} G_{d a}\right] .
$$

Los dos primeros términos no contribuirán a la amplitud de dispersión pues el gravitón es de traza nula.

\section{Término de contacto}

El término de contacto da una amplitud de la forma

$$
\begin{aligned}
& A_{c}^{\text {vector }}\left(h_{1}, h_{4}, \epsilon_{2}, \epsilon_{3}\right)= \\
& -i T_{7} \kappa^{2}\left\{2\left(h_{1} h_{4}\right)\left[\left(\epsilon_{2} k_{3}\right)\left(\epsilon_{3} k_{2}\right)+\left(k_{2} k_{3}\right)\left(\epsilon_{2} \epsilon_{3}\right)\right]\right. \\
& +4\left[\left(k_{2} h_{1} k_{3}\right)\left(\epsilon_{2} h_{4} \epsilon_{3}\right)+\left(\epsilon_{2} h_{1} \epsilon_{3}\right)\left(k_{2} h_{4} k_{3}\right)-\left(\epsilon_{2} h_{1} k_{3}\right)\left(\epsilon_{3} h_{4} k_{2}\right)-\left(\epsilon_{3} h_{1} k_{2}\right)\left(\epsilon_{2} h_{4} k_{3}\right)\right] \\
& +4\left[\left(k_{2} k_{3}\right)\left(\epsilon_{2} h_{1} h_{4} \epsilon_{3}\right)+\left(k_{2} k_{3}\right)\left(\epsilon_{3} h_{1} h_{4} \epsilon_{2}\right)+\left(\epsilon_{2} \epsilon_{3}\right)\left(k_{2} h_{1} h_{4} k_{3}\right)+\left(\epsilon_{2} \epsilon_{3}\right)\left(k_{3} h_{1} h_{4} k_{2}\right)\right] \\
& \left.-4\left[\left(\epsilon_{2} k_{3}\right)\left(\epsilon_{3} h_{1} h_{4} k_{2}\right)\left(\epsilon_{2} k_{3}\right)\left(k_{2} h_{1} h_{4} \epsilon_{3}\right)+\left(\epsilon_{3} k_{2}\right)\left(\epsilon_{2} h_{1} h_{4} k_{3}\right)+\left(\epsilon_{3} k_{2}\right)\left(k_{3} h_{1} h_{4} \epsilon_{2}\right)\right]\right\} .
\end{aligned}
$$

No existen términos de la forma $\left(\epsilon_{2} h_{1} k_{2}\right)$, pues solo podrían venir de un término proporcional a $h^{a b} G_{a b}$ en $L_{h h B B}$. Sin embargo, este término se anula trivialmente por ser el gravitón simétrico y el tensor de campo antisimétrico en los índices.

\footnotetext{
${ }^{22}$ Recordemos que reservamos la notación $F_{m n}=\partial_{m} A_{n}-\partial_{n} A_{m}$ para el campo de gauge proveniente del gravitón elegido como (6.1.11).
} 

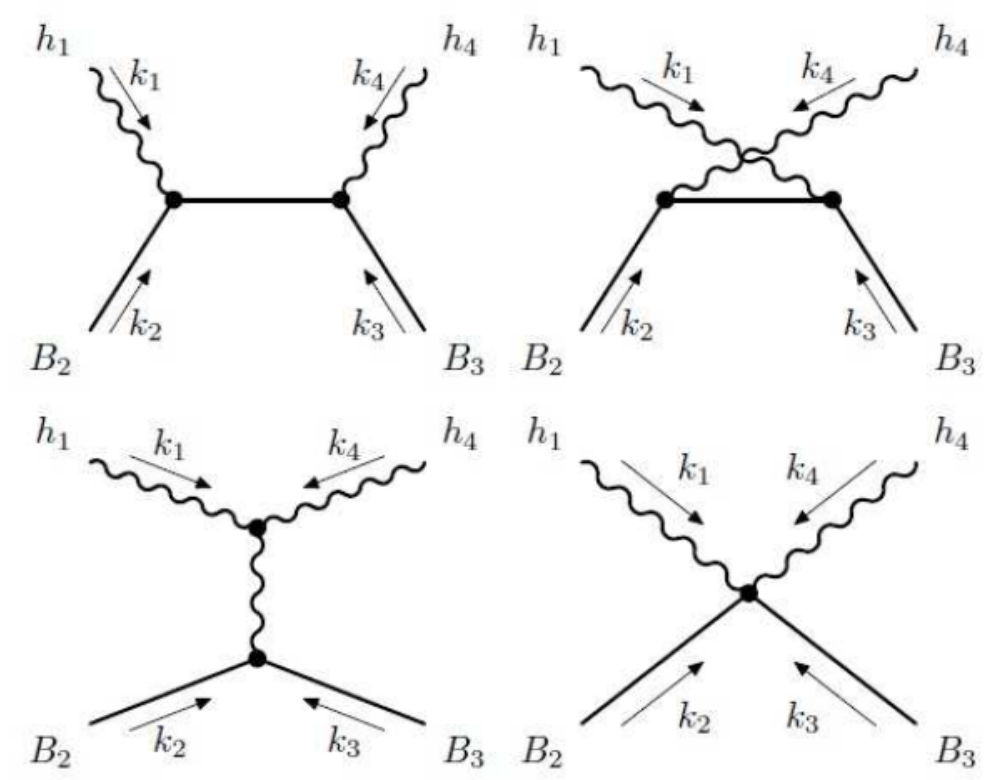

Figura 7.7: Diagramas de Feynman correspondientes al cálculo de supergravedad para el DIS de mesones vectoriales. Las líneas onduladas representan los gravitones -duales holográficos de los fotones virtuales- mientras que las contínuas representan cuerdas abiertas -duales holográficos de los mesones vectoriales-.

\section{Canales $s$ y $u$}

Para calcular estos diagramas mostrados en la Figura 7.7 solo hace falta conocer el vértice de interacción entre un gravitón y dos mesones vectoriales, proveniente de $L_{h B B}$, y el propagador de estos últimos, obtenido del término cinético $L_{B B}$. Calculándolos de manera análoga a la Sección anterior resultan

$$
L_{B B}=-\frac{T_{7}}{4} G^{a b} G_{a b}, L_{h B B}=T_{7} \kappa\left[\frac{1}{4} h G^{a b} G_{a b}+h^{a b} G_{b c} G_{a}^{c}\right] .
$$

Del término cinético para $B_{a}$, su propagador resulta $D_{a b}(k)=-i \eta_{a b} /\left(T_{7} k^{2}\right)$. El vértice de tres puntos es, considerando que todos nuestros gravitones son físicos,

$$
V_{h B B}^{a b, c, d}\left(k_{2}, k_{3}\right)=2 i \kappa T_{7}\left[\eta^{d b} k_{3}^{c} k_{2}^{a}+\eta^{c b} k_{2}^{d} k_{3}^{a}-\eta^{c d} k_{2}^{a} k_{3}^{b}-\left(k_{2} \cdot k_{3}\right) \eta^{c a} \eta^{d b}\right],
$$

donde $k_{2}$ y $k_{3}$ son los momentos entrantes de los mesones vectoriales.

Uniendo el vértice $V_{h B B}$ con un propagador de $B_{a}$ construimos el diagrama de Feynman del canal $s$. La amplitud de este es

$$
\begin{aligned}
& A_{s}\left(h_{1}, h_{4}, \epsilon_{2}, \epsilon_{3}\right)=h_{1 g k} h_{4 c d} \epsilon_{2 e} \epsilon_{3 f} V_{h A A}^{g k, e, a}\left(k_{2},-k_{1}-k_{2}\right) D_{a b}\left(k_{1}+k_{2}\right) V_{h A A}^{c d, b, f}\left(k_{1}+k_{2}, k_{3}\right) \\
& =-\frac{4 i \kappa^{2} T_{7}}{s}\left\{-\frac{1}{2}\left[t\left(k_{2} h_{1} \epsilon_{2}\right)\left(k_{3} h_{4} \epsilon_{3}\right)+s\left[\left(k_{2} h_{1} k_{2}\right)\left(\epsilon_{2} h_{4} \epsilon_{3}\right)-\left(k_{3} h_{1} \epsilon_{2}\right)\left(k_{3} h_{4} \epsilon_{3}\right)\right.\right.\right. \\
& \left.\left.+\left(\epsilon_{2} h_{1} \epsilon_{3}\right)\left(k_{3} h_{4} k_{3}\right)\right]\right]+\frac{1}{2} s\left[\left(\epsilon_{2} k_{1}\right)\left(k_{2} h_{1} h_{4} \epsilon_{3}\right)+\left(\epsilon_{3} k_{4}\right)\left(\epsilon_{2} h_{1} h_{4} k_{3}\right)\right]+\left(\epsilon_{2} k_{1}\right)\left(\epsilon_{3} k_{4}\right)\left(k_{2} h_{1} h_{4} k_{3}\right) \\
& +\left(k_{2} h_{1} \epsilon_{2}\right)\left[\left(\epsilon_{3} k_{4}\right)\left(k_{2} h_{4} k_{3}\right)-\left(\epsilon_{3} k_{2}\right)\left(k_{3} h_{4} k_{3}\right)\right]+\left(\epsilon_{2} k_{1}\right)\left(k_{2} h_{1} k_{3}\right)\left[\left(k_{3} h_{4} \epsilon_{3}\right)-\left(k_{3} h_{4} k_{3}\right)\right] \\
& \left.+\left(k_{2} h_{1} k_{2}\right)\left[\left(\epsilon_{2} \epsilon_{3}\right)\left(k_{3} h_{4} k_{3}\right)-\left(\epsilon_{2} k_{3}\right)\left(k_{3} h_{4} \epsilon_{3}\right)-\left(\epsilon_{3} k_{4}\right)\left(k_{3} h_{4} \epsilon_{2}\right)\right]+\frac{1}{4} s^{2}\left(\epsilon_{2} h_{1} h_{4} \epsilon_{3}\right)\right\} .
\end{aligned}
$$


La amplitud para el canal $u$ es muy similar y se obtiene de la anterior mediante el intercambio

$$
A_{u}\left(h_{1}, h_{4}, \epsilon_{2}, \epsilon_{3}\right)=A_{s}(2 \leftrightarrow 3, u \leftrightarrow s) .
$$

\section{El canal $t$}

Este diagrama, mostrado en la Figura 7.7, será el más importante en el presente régimen. Para construirlo necesitamos tres ingredientes: el vértice de tres gravitones derivado de $L_{h h h}$, el propagador del graviton proveniente de $L_{h h}^{\partial^{2}}$ y el vértice de interacción de un gravitón y dos mesones vectoriales, de $L_{h B B}$.

El gravitón que conecta los vértices provenientes de $L_{h h h}$ y de $L_{h B B}$ es virtual, por lo que no podemos despreciar el primer término, que contiene su traza. Obtenemos el vértice de tres puntos

$$
\begin{aligned}
& V_{h B B}^{a b, c, d}\left(k_{1}, k_{2}\right)=2 i T_{7} \kappa \times \\
& {\left[\eta^{d b} k_{2}^{c} k_{1}^{a}+\eta^{c b} k_{1}^{d} k_{2}^{a}-\eta^{c d} k_{1}^{a} k_{2}^{b}-\left(k_{1} \cdot k_{2}\right) \eta^{c a} \eta^{d b}+\frac{1}{2} \eta^{a b}\left(\eta^{c d}\left(k_{1} \cdot k_{2}\right)-k_{1}^{c} k_{2}^{d}\right)\right] .}
\end{aligned}
$$

Contrayendo este resultado con $(7.2 .26$ resulta una amplitud que esquemáticamente se escribe

$$
A_{t}=h_{1 g k} h_{4 c d} S_{a b}^{g k, c d}\left(k_{1}, k_{4}\right) V_{h B B}^{a b, e, f}\left(k_{2}, k_{3}\right) \epsilon_{2 e} \epsilon_{3 f} .
$$

Explícitamente será

$$
\begin{aligned}
& A_{t}=\frac{4 i \kappa^{2} T_{7}}{t} \times \frac{1}{2}\left(h_{1} h_{4}\right)\left[\left(s+\frac{1}{2} u\right)\left[\left(\epsilon_{2} k_{3}\right)\left(\epsilon_{3} k_{1}\right)+\left(\epsilon_{2} k_{4}\right)\left(\epsilon_{3} k_{2}\right)\right]\right. \\
& +\left(u+\frac{1}{2} s\right)\left[\left(\epsilon_{2} k_{3}\right)\left(\epsilon_{3} k_{4}\right)+\left(\epsilon_{2} k_{1}\right)\left(\epsilon_{3} k_{2}\right)\right]-\frac{1}{2} t\left[2\left(\epsilon_{2} k_{1}\right)\left(\epsilon_{3} k_{1}\right)+2\left(\epsilon_{2} k_{4}\right)\left(\epsilon_{3} k_{4}\right)\right. \\
& \left.\left.+\left(\epsilon_{2} k_{4}\right)\left(\epsilon_{3} k_{1}\right)+\left(\epsilon_{2} k_{1}\right)\left(\epsilon_{3} k_{4}\right)+3\left(\epsilon_{2} k_{3}\right)\left(\epsilon_{3} k_{2}\right)\right]-\frac{1}{2}\left(\epsilon_{2} \epsilon_{3}\right)\left[u^{2}+s^{2}+s u\right]\right] \\
& +\left(\epsilon_{2} \epsilon_{3}\right)\left[\left(k_{2} h_{1} k_{3}\right)\left(k_{1} h_{4} k_{1}\right)+\left(k_{4} h_{1} k_{4}\right)\left(k_{2} h_{4} k_{3}\right)-\left(k_{4} h_{1} k_{3}\right)\left(k_{2} h_{4} k_{1}\right)\right. \\
& -\left(k_{4} h_{1} k_{2}\right)\left(k_{3} h_{4} k_{1}\right)+\frac{1}{2} u\left[\left(k_{2} h_{1} h_{4} k_{1}\right)+\left(k_{4} h_{1} h_{4} k_{3}\right)\right]+\frac{1}{2} s\left[\left(k_{3} h_{1} h_{4} k_{1}\right)+\left(k_{4} h_{1} h_{4} k_{2}\right)\right] \\
& \left.-\frac{1}{2} t\left[\left(k_{3} h_{1} h_{4} k_{2}\right)+\left(k_{2} h_{1} h_{4} k_{3}\right)+\left(k_{4} h_{1} h_{4} k_{1}\right)\right]\right]-\frac{1}{2} t\left[\left(\epsilon_{2} h_{1} \epsilon_{3}\right)\left(k_{1} h_{4} k_{1}\right)+\left(k_{4} h_{1} k_{4}\right)\left(\epsilon_{2} h_{4} \epsilon_{3}\right)\right] \\
& +\frac{1}{4} t^{2}\left[\left(\epsilon_{2} h_{1} h_{4} \epsilon_{3}\right)+\left(\epsilon_{3} h_{1} h_{4} \epsilon_{2}\right)\right]+\frac{1}{2}\left(\epsilon_{2} k_{3}\right)\left[-2\left(k_{4} h_{1} k_{4}\right)\left(\epsilon_{3} h_{4} k_{2}\right)-2\left(\epsilon_{3} h_{1} k_{2}\right)\left(k_{1} h_{4} k_{1}\right)\right. \\
& +2\left(k_{4} h_{1} k_{2}\right)\left(\epsilon_{3} h_{4} k_{1}\right)+2\left(\epsilon_{3} h_{1} k_{4}\right)\left(k_{1} h_{4} k_{2}\right)+t\left(k_{2} h_{1} h_{4} \epsilon_{3}\right)+t\left(\epsilon_{3} h_{1} h_{4} k_{2}\right)-s\left(\epsilon_{3} h_{1} h_{4} k_{1}\right) \\
& \left.-u\left(k_{4} h_{1} h_{4} \epsilon_{3}\right)\right]+\frac{1}{2}\left(\epsilon_{3} k_{2}\right)\left[-2\left(k_{4} h_{1} k_{4}\right)\left(\epsilon_{2} h_{4} k_{3}\right)-2\left(\epsilon_{2} h_{1} k_{3}\right)\left(k_{1} h_{4} k_{1}\right)+2\left(k_{4} h_{1} k_{3}\right)\left(\epsilon_{2} h_{4} k_{1}\right)\right. \\
& \left.+2\left(\epsilon_{2} h_{1} k_{4}\right)\left(k_{1} h_{4} k_{3}\right)+t\left(k_{3} h_{1} h_{4} \epsilon_{2}\right)+t\left(\epsilon_{2} h_{1} h_{4} k_{3}\right)-u\left(\epsilon_{2} h_{1} h_{4} k_{1}\right)-s\left(k_{4} h_{1} h_{4} \epsilon_{2}\right)\right] \\
& +\frac{1}{2}\left[\left(\epsilon_{2} h_{1} h_{4} k_{1}\right)\left(\epsilon_{3} k_{1}\right)+\left(\epsilon_{3} h_{1} h_{4} k_{1}\right)\left(\epsilon_{2} k_{1}\right)+\left(k_{4} h_{1} h_{4} \epsilon_{2}\right)\left(\epsilon_{3} k_{4}\right)+\left(k_{4} h_{1} h_{4} \epsilon_{3}\right)\left(\epsilon_{2} k_{4}\right)\right. \\
& \left.+\left(\epsilon_{2} h_{1} k_{4}\right)\left(\epsilon_{3} h_{4} k_{1}\right)+\left(\epsilon_{3} h_{1} k_{4}\right)\left(\epsilon_{2} h_{4} k_{1}\right)\right]+\left[\left(k_{3} h_{1} h_{4} k_{1}\right)\left(\epsilon_{2} k_{4}\right)\left(\epsilon_{3} k_{2}\right)+\left(k_{2} h_{1} h_{4} k_{1}\right)\left(\epsilon_{2} k_{3}\right)\left(\epsilon_{3} k_{4}\right)\right. \\
& \left.+\left(k_{2} h_{4} h_{1} k_{4}\right)\left(\epsilon_{2} k_{3}\right)\left(\epsilon_{3} k_{1}\right)+\left(k_{3} h_{4} h_{1} k_{4}\right)\left(\epsilon_{2} k_{1}\right)\left(\epsilon_{3} k_{2}\right)-\left(k_{4} h_{1} h_{4} k_{1}\right)\left(\epsilon_{2} k_{3}\right)\left(\epsilon_{3} k_{2}\right)\right]
\end{aligned}
$$

De aquí puede chequearse en parte viendo que si conservamos solamente los términos que incluyen el factor $\left(\epsilon_{2} \epsilon_{3}\right)$, recuperamos el resultado de la Sección anterior para mesones escalares en (7.2.27). Lo mismo podemos decir de las amplitudes $A_{c}, A_{s}$ y $A_{u}$, pues el cálculo de teoría de cuerdas resulta ser el mismo, amén de la polarización de los mesones (paralelos a la D7-brana en el caso escalar y perpendiculares a esta en el caso vectorial). 


\subsubsection{Lagrangiano efectivo}

El procedimiento será aquí similar al utilizado para mesones escalares, aunque los cálculos se volverán más complicados. Vamos a listar primero los términos necesarios para reproducir el coeficiente del coeficiente del polo del gravitón en el canal $t$. Este proceso involucra dos gravitones y dos mesones vectoriales como campos externos. Estos últimos son modos no masivos de cuerdas abiertas con polarizaciones paralelas al volumen de mundo de la D7-brana de sabor. Nos encontraremos con dos clases de términos: unos serán similares a los obtenidos en el caso de mesones escalares en la Subsección 7.2.3, con la única diferencia de que poseen aquí un factor extra $\left(\epsilon_{2} \epsilon_{3}\right)$. La segunda clase de términos es nueva y viene dada, junto con sus contribuciones a la amplitud, por ${ }^{23}$

$$
\begin{aligned}
& \mathcal{L}_{1}=\partial_{a} B_{b} \partial^{b} B^{a} \partial^{d} h^{e f} \partial_{d} h_{e f} \rightarrow-2 t\left(h_{1} h_{4}\right)\left(\epsilon_{2} k_{3}\right)\left(\epsilon_{3} k_{2}\right) \\
& \mathcal{L}_{2}=\partial_{a} B_{b} \partial^{b} B^{a} \partial^{d} h_{e f} \partial_{f} h_{d e} \rightarrow 4\left(\epsilon_{2} k_{3}\right)\left(\epsilon_{3} k_{2}\right)\left(k_{4} h_{1} h_{4} k_{1}\right) \\
& \mathcal{L}_{3}=\partial_{a} B^{d} \partial^{a} B^{e} \partial_{d} h^{c f} \partial_{e} h_{c f} \rightarrow-t\left(h_{1} h_{4}\right)\left[\left(\epsilon_{2} k_{1}\right)\left(\epsilon_{3} k_{4}\right)+\left(\epsilon_{2} k_{4}\right)\left(\epsilon_{3} k_{1}\right)\right] \\
& \mathcal{L}_{4}=\partial_{a} B^{d} \partial^{a} B^{e} \partial_{d} h^{c f} \partial_{c} h_{e f} \rightarrow-\frac{1}{2} t\left[\left(\epsilon_{2} k_{1}\right)\left(k_{4} h_{1} h_{4} \epsilon_{3}\right)+\left(\epsilon_{3} k_{1}\right)\left(k_{4} h_{1} h_{4} \epsilon_{2}\right)\right. \\
& \left.+\left(\epsilon_{2} k_{4}\right)\left(\epsilon_{3} h_{1} h_{4} k_{1}\right)+\left(\epsilon_{3} k_{4}\right)\left(\epsilon_{2} h_{1} h_{4} k_{1}\right)\right] \\
& \mathcal{L}_{5}=\partial_{a} B^{d} \partial^{a} B^{e} \partial^{c} h_{d f} \partial_{c} h_{e}^{f} \rightarrow \frac{1}{2} t^{2}\left[\left(\epsilon_{2} h_{1} h_{4} \epsilon_{3}\right)+\left(\epsilon_{3} h_{1} h_{4} \epsilon_{2}\right)\right] \\
& \mathcal{L}_{6}=\partial_{a} B^{d} \partial^{a} B^{e} \partial^{c} h_{d f} \partial^{f} h_{e c} \rightarrow-t\left[\left(\epsilon_{2} h_{1} k_{4}\right)\left(\epsilon_{3} h_{4} k_{1}\right)+\left(\epsilon_{3} h_{1} k_{4}\right)\left(\epsilon_{2} h_{4} k_{1}\right)\right] \\
& \mathcal{L}_{7}=\partial^{d} B_{a} \partial^{a} B^{e} \partial_{d} h^{c f} \partial_{e} h_{c f} \rightarrow \frac{1}{2}\left(h_{1} h_{4}\right)\left[-s\left(\left(\epsilon_{2} k_{1}\right)\left(\epsilon_{3} k_{2}\right)+\left(\epsilon_{2} k_{3}\right)\left(\epsilon_{3} k_{4}\right)\right)\right. \\
& \left.-u\left(\left(\epsilon_{2} k_{3}\right)\left(\epsilon_{3} k_{1}\right)+\left(\epsilon_{2} k_{4}\right)\left(\epsilon_{3} k_{2}\right)\right)\right] \\
& \mathcal{L}_{8}=\partial^{d} B_{a} \partial^{a} B^{e} \partial^{c} h_{d f} \partial_{c} h_{e}^{f} \rightarrow-\frac{1}{2} t\left[\left(\epsilon_{2} k_{3}\right)\left(\left(\epsilon_{3} h_{1} h_{4} k_{2}\right)+\left(k_{2} h_{1} h_{4} \epsilon_{3}\right)\right)\right. \\
& \left.+\left(\epsilon_{3} k_{2}\right)\left(\left(\epsilon_{2} h_{1} h_{4} k_{3}\right)+\left(k_{3} h_{1} h_{4} \epsilon_{2}\right)\right)\right] \\
& \mathcal{L}_{9}=\partial^{d} B_{a} \partial^{a} B^{e} \partial^{c} h_{d f} \partial^{f} h_{e c} \rightarrow\left(\epsilon_{2} k_{3}\right)\left[\left(\epsilon_{3} h_{1} k_{4}\right)\left(k_{2} h_{4} k_{1}\right)+\left(k_{2} h_{1} k_{4}\right)\left(\epsilon_{3} h_{4} k_{1}\right)\right] \\
& +\left(\epsilon_{3} k_{2}\right)\left[\left(\epsilon_{2} h_{1} k_{4}\right)\left(k_{3} h_{4} k_{1}\right)+\left(k_{3} h_{1} k_{4}\right)\left(\epsilon_{2} h_{4} k_{1}\right)\right] \\
& \mathcal{L}_{10}=\partial^{d} B_{a} \partial^{a} B^{e} \partial_{d} h^{c f} \partial_{c} h_{e f} \rightarrow-\frac{1}{2} s\left[\left(\epsilon_{2} k_{3}\right)\left(k_{4} h_{1} h_{4} \epsilon_{3}\right)+\left(\epsilon_{3} k_{2}\right)\left(\epsilon_{2} h_{1} h_{4} k_{1}\right)\right] \\
& -\frac{1}{2} u\left[\left(\epsilon_{2} k_{3}\right)\left(\epsilon_{3} h_{1} h_{4} k_{1}\right)+\left(\epsilon_{3} k_{2}\right)\left(k_{4} h_{1} h_{4} \epsilon_{2}\right)\right] \\
& \mathcal{L}_{11}=\partial^{d} B_{a} \partial^{a} B^{e} \partial_{e} h^{c f} \partial_{c} h_{d f} \quad \rightarrow \quad\left(\epsilon_{2} k_{3}\right)\left[\left(\epsilon_{3} k_{1}\right)\left(k_{4} h_{1} h_{4} k_{2}\right)+\left(\epsilon_{3} k_{4}\right)\left(k_{2} h_{1} h_{4} k_{1}\right)\right] \\
& +\left(\epsilon_{3} k_{2}\right)\left[\left(\epsilon_{2} k_{1}\right)\left(k_{4} h_{1} h_{4} k_{3}\right)+\left(\epsilon_{2} k_{4}\right)\left(k_{3} h_{1} h_{4} k_{1}\right)\right] \\
& \mathcal{L}_{12}=\partial_{a} B^{d} \partial^{a} B^{e} h^{f g} \partial_{f} \partial_{g} h_{d e} \rightarrow-t\left[\left(\epsilon_{2} h_{1} \epsilon_{3}\right)\left(k_{1} h_{4} k_{1}\right)+\left(k_{4} h_{1} k_{4}\right)\left(\epsilon_{2} h_{4} \epsilon_{3}\right)\right] \\
& \mathcal{L}_{13}=\partial^{d} B_{a} \partial^{a} B^{e} h^{f g} \partial_{f} \partial_{g} h_{d e} \rightarrow\left(\epsilon_{2} k_{3}\right)\left[\left(k_{4} h_{1} k_{4}\right)\left(\epsilon_{3} h_{4} k_{2}\right)+\left(\epsilon_{3} h_{1} k_{2}\right)\left(k_{1} h_{4} k_{1}\right)\right] \\
& +\left(\epsilon_{3} k_{2}\right)\left[\left(k_{4} h_{1} k_{4}\right)\left(\epsilon_{2} h_{4} k_{3}\right)+\left(\epsilon_{2} h_{1} k_{3}\right)\left(k_{1} h_{4} k_{1}\right)\right] \\
& \mathcal{L}_{14}=\partial_{a} B^{d} \partial^{a} B^{e} h^{f g} \partial_{d} \partial_{e} h_{f g} \rightarrow-t\left(h_{1} h_{4}\right)\left[\left(\epsilon_{2} k_{1}\right)\left(\epsilon_{3} k_{1}\right)+\left(\epsilon_{2} k_{4}\right)\left(\epsilon_{3} k_{4}\right)\right] .
\end{aligned}
$$

A partir de estos términos y utilizando conservación de momentos y propagación transversal de estados físicos, el lagrangiano efectivo correspondiente al canal $t$ es, a menos de un factor

\footnotetext{
${ }^{23}$ Esta lista no es exhaustiva en cuanto a los posibles términos que podrían aparecer, sino que solamente listamos aquellos necesarios para la construcción de un lagrangiano de interacción efectivo de cuatro puntos para mesones vectoriales que dé cuenta de la amplitud del canal $t$.
} 


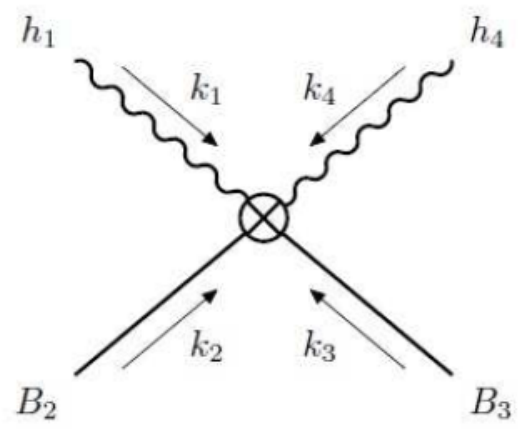

Figura 7.8: Lagrangiano efectivo de interacción de cuatro puntos. Las líneas onduladas representan gravitones físicos mientras que las continuas representan cuerdas abiertas -duales holográficos de los mesones vectoriales-.

global 24

$$
\begin{aligned}
&-\frac{1}{4} \times \mathcal{L}_{1}-\frac{1}{4} \times \mathcal{L}_{2}+\frac{1}{4} \times \mathcal{L}_{3}+1 \times \mathcal{L}_{4}+\frac{1}{2} \times \mathcal{L}_{5}-\frac{1}{2} \times \mathcal{L}_{6}+\frac{1}{2} \times \mathcal{L}_{7}-1 \times \mathcal{L}_{8}+1 \times \mathcal{L}_{9} \\
&-1 \times \mathcal{L}_{10}+1 \times \mathcal{L}_{11}+\frac{1}{2} \times \mathcal{L}_{12}-1 \times \mathcal{L}_{13}+\frac{1}{2} \times \mathcal{L}_{14}
\end{aligned}
$$

Esta forma esquemática no incluye la parte análoga al lagrangiano para mesones escalares 7.2.29), así como tampoco los términos proporcionales a $\left(k_{1} h_{4} k_{1}\right)$ y $\left(k_{4} h_{1} k_{4}\right)$, que se anulan ante la forma (6.1.11) elegida para el gravitón. La interacción efectiva se esquematiza en la Figura 7.8 .

En el régimen cinemático en el que estamos interesados, $0<\alpha^{\prime} t \ll 1 \ll \alpha^{\prime} s$, el primer orden en la amplitud del DIS para $x$ pequeño será de la forma $\frac{s^{2}}{t}$. Esto equivale a considerar términos proporcionales a $s^{2}$ en la amplitud del canal $t$. Hay un solo término con estas propiedades para el caso de mesones vectoriales, así como sucedía para mesones escalares y glueballs. Este es el término proporcional a $\left(h_{1} h_{4}\right) \frac{s^{2}}{t}$. En el caso de mesones vectoriales hay un segundo tipo de término que podría aparecer, proporcional a $s^{2}\left[\left(\epsilon_{2} h_{1} h_{4} e_{3}\right)+\left(\epsilon_{3} h_{1} h_{4} \epsilon_{2}\right)\right]$, pero este no está presente en la amplitud del canal $t$. Por lo tanto, a primer orden en esta aproximación todos los términos del lagrangiano efectivo son subdominantes en salvo el proporcional a $\left(h_{1} h_{4}\right) \frac{s^{2}}{t}$. En caso de que quisiéramos considerar los términos de orden $\frac{s}{t}$, el único relevante es

$$
\partial_{a} B_{c} \partial^{b} B^{c} \partial^{a} h^{n q} \partial_{b} h_{n q}=\partial_{a} B_{c} \partial^{b} B^{c} \partial^{a} A^{n} \partial_{b} A_{n} v_{i} v^{i} .
$$

Ahora bien, como quisiéramos escribir un lagrangiano efectivo invariante de gauge, usamos $G_{a b}=$ $\partial_{a} B_{b}-\partial_{b} B_{a}$ y $F_{m n}=\partial_{m} A_{n}-\partial_{n} A_{m}$, con lo que resulta

$$
\mathcal{L}_{e f f} \propto G_{a c} G^{b c} F^{a n} F_{b n},
$$

al que faltaría multiplicar por un factor global.

\subsubsection{Cálculo del tensor hadrónico}

Obtendremos aquí el tensor hadrónico para mesones vectoriales polarizados para acoplamiento fuerte y valores pequeños de $x$, así como su descomposición en funciones de estructura. Lo obtendremos a partir del lagrangiano efectivo (7.3.13).

\footnotetext{
${ }^{24}$ Existen también otros lagrangianos equivalentes. Estos pueden obtenerse utilizando la conservación del momento total.
} 
Recordemos que en la amplitud de cuatro puntos de teoría de cuerdas $\mathcal{A}_{4}^{202 c, v e c t o r}$ solo es relevante el primer término para nuestro régimen cinemático de interés. Entonces tenemos

$$
\mathcal{A}_{4}^{202 c, \text { vector }} \simeq \mathcal{P}_{1}^{202 c, \text { vector }} \mathcal{K}_{1}^{202 c, \text { vector }},
$$

con $\mathcal{K}_{1}^{2 o 2 c, v e c t o r}$ dado por 7.3 .13 , mientras que podemos tomar $\mathcal{P}_{1}^{202 c, v e c t o r}=\mathcal{P}_{1}^{202 c, s c a l a r}$, es decir, el mismo prefactor que en el caso del mesón escalar. Este está asociado con la dependencia genérica de las amplitudes en los invariantes cinemáticos $s \approx-u \mathrm{y} t$ en el régimen $t \ll s$, y con el intercambio de modos excitados de cuerdas. La expresión equivalente a 7.2 .32 para este caso es

$$
n_{\mu}^{*} n_{\nu} I m_{e x c} T^{\mu \nu}=\frac{\pi \alpha^{\prime}}{8} \sum_{m=1}^{\infty} \int d \Omega_{3} d r \sqrt{-g} v_{i} v^{i} G^{* m q}(P) G_{q}^{n}(P) F_{m p}^{*}(q) F_{n}^{p}(q) \delta\left(m-\frac{\alpha^{\prime} \tilde{s}}{4}\right),
$$

donde todos los índices están contraídos con la métrica completa de diez dimensiones, consistentemente con nuestra notación de índices. Omitimos aquí nuevamente las lintegrales en el espaciotiempo de Minkowski $\left(x_{0}, \cdots, x_{3}\right)$ pues solo aportan la delta de conservación del momento. Las soluciones a las ecuaciones de movimiento para los campos de interés son (ver [1] y Capítulo 6)

$$
\begin{aligned}
A_{\mu}(q) & =n_{\mu} f(r) e^{i q \cdot x} \\
A_{r}(q) & =\frac{-i q \cdot n}{q^{2}} f^{\prime}(r) e^{i q \cdot x} \\
B_{\mu}^{l} & =\frac{\zeta_{\mu}}{\Lambda} \frac{c_{i}^{l}}{\Lambda R^{3}}\left(\frac{r}{\Lambda R^{2}}\right)^{-\Delta} Y^{l}\left(\Omega_{3}\right) e^{i P \cdot x}=\frac{\zeta_{\mu}}{\Lambda} X^{l} \\
B_{r}^{l} & =0
\end{aligned}
$$

donde las dos primeras líneas corresponden al campo de gauge $A_{m}$, y coinciden con el caso de la Sección 7.1 y la Sección 7.2 . Al igual que en el caso de los mesones escalares definimos $\omega=\frac{q R^{2}}{r}$, con lo que escribimos $f=\omega K_{1}(\omega)$, con $K_{1}$ la función de Bessel modificada de segunda especie. Omitimos a partir de aquí el índice $l$ que identifica a cada mesón de la torre de Kaluza-Klein.

El tensor de campo para $B_{a}$ es 25

$$
\begin{aligned}
G_{\mu \nu} & =i\left(P_{\mu} B_{\nu}-P_{\nu} B_{\mu}\right)=\frac{i}{\Lambda}\left(P_{\mu} \zeta_{\nu}-P_{\nu} \zeta_{\mu}\right) X \\
G_{\mu r} & =-\partial_{r} B_{\mu}=\frac{\Delta}{\Lambda r} \zeta_{\mu} X, \quad G_{\mu i}=-\partial_{i} B_{\mu}=\frac{\zeta_{\mu}}{\Lambda} \partial_{i} X
\end{aligned}
$$

de donde construimos las diferentes contracciones que aparecen en el lagrangiano efectivo

$$
\begin{aligned}
G_{r q}^{*} G_{r}^{q} & =\frac{R^{2}}{r^{2}} \eta^{\rho \sigma} G_{r \rho}^{*} G_{r \sigma}=\frac{R^{2} \Delta^{2}}{\Lambda^{2} r^{4}}\left(\zeta \cdot \zeta^{*}\right) X X^{*} \\
G_{\mu q}^{*} G_{r}^{q} & =\frac{R^{2}}{r^{2}} \eta^{\rho \sigma} G_{\mu \rho}^{*} G_{r \sigma}=i \frac{R^{2} \Delta}{\Lambda^{2} r^{3}}\left(\zeta \cdot \zeta^{*}\right) X X^{*} P_{\mu} \\
G_{\mu q}^{*} G_{\nu}^{q} & =\frac{R^{2}}{r^{2}} \eta^{\rho \sigma} G_{\mu \rho}^{*} G_{\nu \sigma}+\frac{r^{2}}{R^{2}} G_{\mu r}^{*} G_{\nu r}+\frac{1}{R^{2}} \tilde{g}^{i j} G_{\mu i} G_{\nu j} \\
& =\frac{R^{2}}{\Lambda^{2} r^{2}}\left(\zeta \cdot \zeta^{*}\right) X X^{*} P_{\mu} P_{\nu}+\left(\frac{R^{2} P^{2}}{r^{2}}+\frac{\Sigma^{2}}{R^{2}}\right) \frac{X X^{*}}{\Lambda^{2}} \zeta_{\mu}^{*} \zeta_{\nu} .
\end{aligned}
$$

\footnotetext{
${ }^{25}$ El tensor de campo para $A_{m}$ es el mismo que en las dos Secciones anteriores.
} 
Hemos definido $\Sigma^{2} \equiv \Delta^{2}+\Delta(\Delta-2)$ debido a la presencia de derivadas en la parte angular ${ }^{26}$ Así como $\Delta$ es función del índice $l$, también $\Sigma$ lo será. Como extraeremos de aquí $T^{\mu \nu}$, todos los términos de la forma $q \cdot n$ y $q \cdot n^{*}$ resultarán en términos proporcionales a $q^{\nu}$ y $q^{\mu}$ respectivamente, por lo que se anularán luego lo contraeremos con el tensor leptónico, como se explicó en el Capítulo 4. Solo resta expandir el lagrangiano efectivo $G^{* m q}(P) G_{q}^{n}(P) F_{m p}^{*}(q) F_{n}^{p}(q)=$ $g^{m u} g^{n v} g^{q q^{\prime}} g^{p p^{\prime}} G_{u q}^{*}(P) G_{v q^{\prime}}(P) F_{m p}^{*}(q) F_{n p^{\prime}}(q)$ en términos de las soluciones para los campos. Podemos expresar este lagrangiano efectivo de manera análoga al caso de los mesones escalares como

$$
\mathcal{L}_{4}^{e f f, v}=\mathcal{L}_{4, A}^{e f f, v}+\mathcal{L}_{4, B}^{e f f, v}+\mathcal{L}_{4, C}^{e f f, v}+\mathcal{L}_{4, D}^{e f f, v}
$$

con

$$
\begin{aligned}
\mathcal{L}_{4, A}^{e f f, v}= & \frac{r^{4}}{R^{4}} G_{r q}^{*} G_{r}^{q} F_{r p}^{*} F_{r}^{p}=\frac{\Delta^{2}}{\Lambda^{2} r^{2}}\left(f^{\prime}\right)^{2}\left(\zeta \cdot \zeta^{*}\right)\left(n \cdot n^{*}\right)|X|^{2} \\
\mathcal{L}_{4, B}^{e f f, v}= & \mathcal{L}_{4, C}^{e f f, v}=\eta^{\mu \nu} G_{\mu q}^{*} G_{r}^{q} F_{\mu p}^{*} F_{r}^{p}=\frac{R^{4} \Delta}{\Lambda^{2} r^{5}} f f^{\prime}\left(\zeta \cdot \zeta^{*}\right)\left(n \cdot n^{*}\right)|X|^{2}(P \cdot q) \\
\mathcal{L}_{4, D}^{e f f, v}= & \frac{R^{4}}{r^{4}} \eta^{\mu \rho} \eta^{\nu \sigma} G_{\rho q}^{*} G_{\sigma}^{q} F_{\mu p}^{*} F_{\nu}^{p}=\frac{R^{4}|X|^{2}}{r^{4} \Lambda^{2}} \times\left[\frac{R^{4}}{r^{4}}\left(\zeta \cdot \zeta^{*}\right)\left(n \cdot n^{*}\right) f^{2}(P \cdot q)^{2}+\right. \\
& \left(\frac{R^{4}}{r^{4}} q^{2} f^{2}+\left(f^{\prime}\right)^{2}\right)\left(\zeta \cdot \zeta^{*}\right)(P \cdot n)\left(P \cdot n^{*}\right)+ \\
& \left(\frac{R^{4}}{r^{4}} P^{2}+\frac{\Sigma^{2}}{r^{2}}\right) f^{2}\left(n \cdot n^{*}\right)(q \cdot \zeta)\left(q \cdot \zeta^{*}\right)+ \\
& \left.\left(\frac{R^{4}}{r^{4}} P^{2} q^{2} f^{2}+P^{2}\left(f^{\prime}\right)^{2}+\frac{\Sigma^{2}}{r^{2}} q^{2} f^{2}+\frac{\Sigma^{2} r^{2}}{R^{4}}\left(f^{\prime}\right)^{2}\right)\left(n^{*} \cdot \zeta^{*}\right)(n \cdot \zeta)\right] .
\end{aligned}
$$

Al igual que en el caso del mesón escalar, los términos $\mathcal{L}_{4, A}^{e f f, v}, \mathcal{L}_{4, B}^{e f f, v}$ y $\mathcal{L}_{4, C}^{e f f, v}$ son subdominantes con respecto a $\mathcal{L}_{4, D}^{\text {eff,v }}$, asociado al factor $n \cdot n^{*}$. Despreciaremos entonces la contribución proveniente de $\mathcal{L}_{4, A}^{e f f, v}, \mathcal{L}_{4, B}^{e f f, v}$ y $\mathcal{L}_{4, C}^{e f f, v}$.

Extrayendo ahora las polarizaciones $n$ y $n^{*}$ se hará visible la estructura tensorial en índices de Lorentz. Obtendremos las piezas $\eta^{\mu \nu}, P^{\mu} P^{\nu}$ y $\zeta^{* \mu} \zeta^{\nu}=\frac{1}{2}\left(\zeta^{* \mu} \zeta^{\nu}+\zeta^{* \nu} \zeta^{\mu}\right)+\frac{1}{2}\left(\zeta^{* \mu} \zeta^{\nu}-\zeta^{* \nu} \zeta^{\mu}\right)$. Esta última contribuye a la parte antisimétrica del tensor hadrónico. También se satisface la condición $\left(\zeta^{*} \cdot \zeta\right)=-P^{2}$. Llegamos con esto a la expresión final

$$
\begin{aligned}
& I m_{e x c} T^{\mu \nu}=\frac{\pi \alpha^{\prime}}{8} \sum_{m=1}^{\infty} \int d \Omega_{3} d r \sqrt{-g_{8}} v^{i} v_{i} \delta\left(m-\frac{\alpha^{\prime} \tilde{s}}{4}\right) \times \\
& P^{\mu} P^{\nu} \times\left[\frac{R^{4}|X|^{2}}{r^{4} \Lambda^{2}}\left(\frac{R^{4}}{r^{4}} q^{2} f^{2}+\left(f^{\prime}\right)^{2}\right)\left(\zeta \cdot \zeta^{*}\right)\right]+\left[\frac{1}{2}\left(\zeta^{* \mu} \zeta^{\nu}+\zeta^{* \nu} \zeta^{\mu}\right)+\frac{1}{2}\left(\zeta^{* \mu} \zeta^{\nu}-\zeta^{* \nu} \zeta^{\mu}\right)\right] \\
& \times \frac{R^{4}|X|^{2}}{r^{4} \Lambda^{2}}\left[\frac{R^{4}}{r^{4}} P^{2} q^{2} f^{2}+P^{2}\left(f^{\prime}\right)^{2}+\frac{\Sigma^{2}}{r^{2}} q^{2} f^{2}+\frac{\Sigma^{2} r^{2}}{R^{4}}\left(f^{\prime}\right)^{2}\right] \\
& +\eta^{\mu \nu} \times \frac{R^{4}|X|^{2}}{r^{4} \Lambda^{2}}\left[\frac{R^{4}}{r^{4}} f^{2}\left(\zeta \cdot \zeta^{*}\right)(P \cdot q)^{2}+\left(\frac{R^{4}}{r^{4}} P^{2}+\frac{\Sigma^{2}}{r^{2}}\right) f^{2}(q \cdot \zeta)\left(q \cdot \zeta^{*}\right)\right. \\
& \left.+\left(\zeta \cdot \zeta^{*}\right)\left(\frac{r^{2} \Delta^{2}}{R^{4}}\left(f^{\prime}\right)^{2}+2 \frac{\Delta}{r} f f^{\prime}(P \cdot q)\right)\right] .
\end{aligned}
$$

De la comparación de esta expresión con la descomposición del tensor hadrónico en 4.3.5 calcularemos las funciones de estructura.

\footnotetext{
${ }^{26}$ Estrictamente, este último paso debería realizarse luego de haber integrado, pues realizamos un reemplazo equivalente a una integración por partes. Sin embargo, el resultado final será el correcto. También usamos la notación $g_{i j}=R^{2} \tilde{g}_{i j}$ y propiedades usuales de los armónicos esféricos.
} 


\section{Nuevas relaciones entre $F_{1}$ y $F_{2}$ para $x$ pequeño (Región $\mathrm{B}$ )}

Utilizamos, al igual que en el caso de la Sección anterior para mesones escalares, una expresión análoga a 7.2.32 para la parte imaginaria de $T_{\mu \nu}$. Esta contiene integrales en la coordenada radial $r$ y en las coordenadas angulares de la $S^{3}$, a saber

$$
\begin{aligned}
& I_{1}=\frac{\pi^{2} \alpha^{\prime}}{4} \sum_{m=1}^{\infty} \frac{R^{4}}{\Lambda^{2}} \Sigma^{2} q^{2} \int d \Omega_{3} d r \sqrt{g_{8}} v^{i} v_{i}|X|^{2} \frac{f^{2}(r)}{r^{6}} \delta\left(m-\frac{\alpha^{\prime} s R^{2}}{4 r^{2}}\right), \\
& I_{0}=\frac{\pi^{2} \alpha^{\prime}}{4} \sum_{m=1}^{\infty} \frac{\Sigma^{2}}{\Lambda^{2}} q^{2} \int d \Omega_{3} d r \sqrt{g_{8}} v^{i} v_{i}|X|^{2} \frac{\left(f^{\prime}(r)\right)^{2}}{r^{2}} \delta\left(m-\frac{\alpha^{\prime} s R^{2}}{4 r^{2}}\right), \\
& \tilde{I}_{1}=\frac{\pi^{2} \alpha^{\prime}}{4} \sum_{m=1}^{\infty} \frac{R^{8}}{\Lambda^{2}} P^{2} q^{4} \int d \Omega_{3} d r \sqrt{g_{8}} v^{i} v_{i}|X|^{2} \frac{f^{2}(r)}{r^{8}} \delta\left(m-\frac{\alpha^{\prime} s R^{2}}{4 r^{2}}\right), \\
& \tilde{I}_{0}=\frac{\pi^{2} \alpha^{\prime}}{4} \sum_{m=1}^{\infty} \frac{R^{4}}{\Lambda^{2}} P^{2} q^{2} \int d \Omega_{3} d r \sqrt{g_{8}} v^{i} v_{i}|X|^{2} \frac{\left(f^{\prime}(r)\right)^{2}}{r^{4}} \delta\left(m-\frac{\alpha^{\prime} s R^{2}}{4 r^{2}}\right) .
\end{aligned}
$$

Los resultados de estas son

$$
\begin{aligned}
& I_{1}=\rho_{3}\left|c_{i}\right|^{2} \frac{\pi^{2} \Sigma^{2}}{4 \Lambda^{2} R^{2}}\left(\frac{\Lambda^{2}}{q^{2}}\right)^{\Delta-1} \frac{1}{\sqrt{4 \pi g_{c} N}} I_{1,2 \Delta+3}, \\
& I_{0}=\rho_{3}\left|c_{i}\right|^{2} \frac{\pi^{2} \Sigma^{2}}{4 \Lambda^{2} R^{2}}\left(\frac{\Lambda^{2}}{q^{2}}\right)^{\Delta-1} \frac{1}{\sqrt{4 \pi g_{c} N}} I_{0,2 \Delta+3}, \\
& \tilde{I}_{1}=\rho_{3}\left|c_{i}\right|^{2} \frac{\pi^{2}}{4 \Lambda^{2} R^{2}}\left(\frac{\Lambda^{2}}{q^{2}}\right)^{\Delta-1} \frac{t}{\sqrt{4 \pi g_{c} N}} I_{1,2 \Delta+5}, \\
& \tilde{I}_{0}=\rho_{3}\left|c_{i}\right|^{2} \frac{\pi^{2}}{4 \Lambda^{2} R^{2}}\left(\frac{\Lambda^{2}}{q^{2}}\right)^{\Delta-1} \frac{t}{\sqrt{4 \pi g_{c} N}} I_{0,2 \Delta+5} .
\end{aligned}
$$

Las integrales $\tilde{I}_{1}$ y $\tilde{I}_{0}$ poseen un factor $t \ll 1$ adicional con respecto a $I_{1}$ e $I_{0}$. Las funciones de estructura resultan

$$
\begin{aligned}
& b_{1}=\frac{(P \cdot q)^{2}}{q^{2}}\left[I_{1}+\tilde{I}_{1}\right]=\frac{1}{4 x^{2}}\left[I_{1}+\tilde{I}_{1}\right]=\frac{I_{1}}{4 x^{2}}+\mathcal{O}(t) \\
& b_{2}=-(P \cdot q)\left[I_{1}+I_{0}+\tilde{I}_{1}+\tilde{I}_{0}\right]=\left(1+\frac{I_{0}}{I_{1}}\right) \frac{I_{1}}{2 x}+\mathcal{O}(t) \\
& b_{3}=-\frac{2}{3} b_{2}=-\left(1+\frac{I_{0}}{I_{1}}\right) \frac{I_{1}}{3 x}+\mathcal{O}(t), \\
& b_{4}=\frac{1}{3} b_{2}=\left(1+\frac{I_{0}}{I_{1}}\right) \frac{I_{1}}{6 x}+\mathcal{O}(t), \\
& g_{2}=\frac{(P \cdot q)^{2}}{2 q^{2}}\left[I_{1}+I_{0}+\tilde{I}_{1}+\tilde{I}_{0}\right]=\left(1+\frac{I_{0}}{I_{1}}\right) \frac{I_{1}}{8 x^{2}}+\mathcal{O}(t) \\
& g_{1}=\mathcal{O}(t)
\end{aligned}
$$

de donde también

$$
\begin{aligned}
& F_{1}=\frac{b_{1}}{3}-\frac{P^{2}}{3(P \cdot q)} b_{2}+\frac{(P \cdot q)^{2}}{P^{2}} \tilde{I}_{1}=\frac{I_{1}}{12 x^{2}}+\mathcal{O}(t) \\
& F_{2}=\frac{b_{2}}{3}-\frac{(P \cdot q) q^{2}}{P^{2}}\left[\tilde{I}_{1}+\tilde{I}_{0}\right]=\frac{q^{2}\left[I_{1}+I_{0}+\tilde{I}_{1}+\tilde{I}_{0}\right]}{6 x}-\frac{q^{2}\left[\tilde{I}_{1}+\tilde{I}_{0}\right]}{2 x P^{2}}=\left(1+\frac{I_{0}}{I_{1}}\right) \frac{I_{1}}{6 x}+\mathcal{O}(t) .
\end{aligned}
$$


Considerando ahora solamente los términos dominantes en la expansión en $t$ obtenemos las relaciones

$$
\begin{gathered}
F_{2}=2 x F_{1}\left(1+\frac{I_{0}}{I_{1}}\right), \\
b_{2}=2 x b_{1}\left(1+\frac{I_{0}}{I_{1}}\right)
\end{gathered}
$$

y dado que $I_{1, n}=\frac{n+1}{n-1} I_{0, n}$ tenemos

$$
1+\frac{I_{1}}{I_{0}}=1+\frac{I_{1,2 \Delta+3}}{I_{0,2 \Delta+3}}=\frac{2 \Delta+3}{\Delta+2} .
$$

La ecuación (7.3.46) reproduce exactamente el resultado obtenido para mesones escalares en la Sección anterior. Las ecuaciones (7.3.46 y 7.3.47) son relaciones tipo Callan-Gross con un nuevo factor adicional.

Además, obtuvimos las nuevas relaciones

$$
b_{1}=3 F_{1}, \quad b_{4}=-\frac{1}{2} b_{3}, \quad g_{1}=0 .
$$

En las Conclusiones discutiremos cómo se comparan estas relaciones con las correspondientes al caso $x \lesssim 1$ estudiado en el Capítulo 6 .

Para la región $\mathbf{C}\left(x \lesssim e^{-\sqrt{\lambda}}\right)$ los cálculos serán realizados en la Subsección 7.5 .

\subsection{Extensión a fondos más generales}

En esta Sección generalizamos los resultados de las Secciones anteriores. Los resultados que presentaremos aquí valen tanto para modelos duales provenientes de teorías de cuerdas tipo IIA como IIB. Estos corresponden a los modelos con Dp-branas de sabor, a saber D3D7 [12, $\mathrm{D} 4 \mathrm{D} 8 \overline{D 8}[14]$ y $\mathrm{D} 4 \mathrm{D} 6 \overline{D 6}$-branas [13]. Partimos de una métrica asintótica general inducida en la $\mathrm{D} p$-brana

$$
d s^{2}=\left(\frac{r}{R}\right)^{\alpha} \eta_{\mu \nu} d x^{\mu} d x^{\nu}+\left(\frac{r}{R}\right)^{\beta}\left[d r^{2}+r^{2} d \Omega_{p-4}^{2}\right],
$$

donde los parámetros dependen del modelo del que se trat ${ }^{27}$

\begin{tabular}{|c|c|c|c|}
\hline Modelo / Parámetro & $\mathrm{p}$ & $\alpha$ & $\beta$ \\
\hline$D 3 D 7$ & 7 & 2 & -2 \\
\hline$D 4 D 8 \overline{D 8}$ & 8 & $3 / 2$ & $-3 / 2$ \\
\hline$D 4 D 6 \overline{D 6}$ & 6 & $3 / 2$ & $-3 / 2$ \\
\hline
\end{tabular}

\footnotetext{
${ }^{27}$ Esta forma general vale para el límite $r \gg L$, ddonde $L$ es algún parámetro dimensional de cada fondo. Es lícito tomar estas formas asintóticas de las métricas pues la interacción en la que estamos interesados ocurre a valores grandes de $r$.
} 
Las soluciones para los campos son (ver [2] y Sección 6.4

$$
\begin{aligned}
X^{l} & =\frac{c_{i}}{\Lambda R^{\frac{p-1}{2}}} e^{i P \cdot x}\left(\frac{r}{R \Lambda^{2}}\right)^{A-\gamma B} Y^{l}\left(S^{p-4}\right), \\
B_{\mu}^{l} & =\frac{\zeta_{\mu}}{\Lambda} X^{l} \\
A_{\mu} & =n_{\mu} e^{i q \cdot y} f(r), \\
A_{r} & =\frac{-i}{q^{2}}(q \cdot n) e^{i q \cdot y} f^{\prime}(r), \\
f(r) & =\Gamma^{-1}(n+1)\left(\frac{q R}{2 B}\right)^{n+1}\left(\frac{r}{R}\right)^{-B(n+1)} K_{n+1}\left[\frac{q R}{B}\left(\frac{r}{R}\right)^{-B}\right],
\end{aligned}
$$

donde $A=\frac{1}{2}(1-\theta), \theta=2 \alpha+(p-3) \beta / 2+(p-4), \gamma^{2}=\frac{A^{2}+l(l+p-5)}{B^{2}}, B=\frac{1}{2}(\alpha-\beta-2) \mathrm{y}$ $n=\frac{2+\beta}{4 B}$. Podemos simplificar la notación definiendo $\omega \equiv \frac{q R}{B}\left(\frac{R}{r}\right)^{B}$, y entonces $f(r)=\Gamma^{-1}(n+$ 1) $\omega^{n+1} K_{n+1}(\omega)$. La relación con los parámetros de la Sección anterior viene dada por $\Delta=$ $\gamma B-A$. Nuevamente, el punto de partida es

$$
n_{\mu}^{*} n_{\nu} \operatorname{Im}_{e x c} T^{\mu \nu}=\frac{\pi \alpha^{\prime}}{8} \sum_{m=1}^{\infty} \int d \Omega_{p-4} d r \sqrt{-g_{p+1}} v_{i} v^{i} G^{* a c}(P) G_{c}^{b}(P) F_{a p}^{*}(q) F_{b}^{p}(q) \delta\left(m-\frac{\alpha^{\prime} \tilde{s}}{4}\right),
$$

y omitimos nuevamente las deltas de Dirac que dictan la conservación del momento total.

En el caso general las funciones de Bessel satisfacen la identidad

$$
\partial_{\omega}\left(\omega^{n+1} K_{n+1}(\omega)\right)=(n+1) \omega^{n} K_{n+1}(\omega)-\omega^{n+1}\left(\frac{n+1}{\omega} K_{n+1}(\omega)+K_{n}(\omega)\right)=-\omega^{n+1} K_{n}(\omega) .
$$

La derivada de $f(r)$ es

$$
f^{\prime}(r)=\frac{1}{\Gamma(n+1)} \frac{d}{d \omega}\left(\omega^{n+1} K_{n+1}(\omega)\right) \frac{d \omega}{d r}=\frac{1}{\Gamma(n+1)} \frac{B}{R}\left(\frac{B \omega}{q R}\right)^{1 / B} \omega^{n+2} K_{n}(\omega) .
$$

Ahora sí podemos obtener las funciones de estructura en esta región. Solo resta calcular la integra 28

$$
\int_{0}^{\infty} d \omega \omega^{D} K_{n}^{2}(\omega)=I_{n, D}=2^{D-2} \frac{\Gamma(\nu+n) \Gamma(\nu-n) \Gamma^{2}(\nu)}{\Gamma(2 \nu)}, \nu=\frac{1}{2}(D+1),
$$

Definiendo integrales análogas a 7.2.50 y 7.2.51),

$$
\begin{aligned}
& I_{1}=\frac{\pi^{2} \alpha^{\prime}}{4} \sum_{m=1}^{\infty} R^{6} q^{2} \int d \Omega_{p-4} d r \sqrt{g_{p+1}} v_{i} v^{i}|X|^{2} \frac{f^{2}(r)}{r^{6}} \delta\left(m-\frac{\alpha^{\prime} s R^{2}}{4 r^{2}}\right) \\
& I_{0}=\frac{\pi^{2} \alpha^{\prime}}{4} \sum_{m=1}^{\infty} R^{2} \int d \Omega_{p-4} d r \sqrt{g_{p+1}} v_{i} v^{i}|X|^{2} \frac{\left(f^{\prime}(r)\right)^{2}}{r^{2}} \delta\left(m-\frac{\alpha^{\prime} s R^{2}}{4 r^{2}}\right) .
\end{aligned}
$$

obtenemos

$$
\begin{aligned}
& I_{1} \propto I_{n+1, D}, I_{0} \propto I_{n, D} \\
D= & 2 n+1+\frac{1}{B}[2 \Delta+\alpha-(\beta / 2+1)(p-5)], \\
& \tilde{I}_{1} \propto I_{n+1, \tilde{D}}, \tilde{I}_{0} \propto I_{n, \tilde{D}} \\
\tilde{D}= & 2 n+3+\frac{1}{B}[2 \Delta+2 \alpha-(\beta / 2+1)(p-3)],
\end{aligned}
$$

\footnotetext{
${ }^{28}$ Este resultado es válido pues los argumentos de las funciones gamma son positivos.
} 
En el caso particular del sistema de D3D7-branas $(\beta=-2)$, el último término se anula. Para el caso de los sistemas de D4D8 $\overline{\mathrm{D} 8}$ y D4D6 $\overline{\mathrm{D} 6}$-branas basta simplemente con poner $\alpha=3 / 2=-\beta$ y $n=1 / 4$.

Todas estas integrales convergen. Por ende, la dependencia en $x$ de las funciones de estructura es la misma que antes y coincide para todos los modelos bajo el alcance de este estudio. Podemos considerar entonces una suerte de comportamiento universal de estas relaciones - (7.3.46), (7.3.47) y 7.3.49)- para las funciones de estructura de mesones escalares y vectoriales polarizados. Lo único que cambiará entre modelos es el factor $\left(1+\frac{I_{1}}{I_{0}}\right)$. Las dos integrales relevantes son

$$
\begin{aligned}
I_{1} & =\frac{\pi^{2} \alpha^{2} \Sigma^{2} \rho_{p-4}\left|c_{i}\right|^{2} q^{4}}{4 \sqrt{4 \pi g_{c} N} \Lambda^{4} \Gamma^{2}(n+1) B}\left(\frac{q R}{B}\right)^{(2 n+1)-D} I_{n+1, D}, \\
I_{0} & =\frac{\pi^{2} \alpha^{2} B \Sigma^{2} \rho_{p-4}\left|c_{i}\right|^{2} q^{2}}{4 \sqrt{4 \pi g_{c} N} \Lambda^{4} R^{2} \Gamma^{2}(n+1)}\left(\frac{q R}{B}\right)^{(2 n+3)-D} I_{n, D},
\end{aligned}
$$

donde definimos ahora $\Sigma^{2} \equiv \Delta^{2}+l(l+p-5)$.

De las expresiones aquí deducidas podemos ver que las relaciones entre funciones de estructura son las mismas que en la Sección anterior, reemplazando los valores de $I_{1}$ e $I_{2}$ por los aquí presentados. Para las relaciones tipo Callan-Gross podemos calcular el factor genérico en términos de los parámetros del modelo particular

$$
1+\frac{I_{0}}{I_{1}}=1+\frac{B^{2}}{q^{2} R^{2}}\left(\frac{q R}{B}\right)^{2} \frac{I_{n, D}}{I_{n+1, D}}=\frac{2 D}{D+2 n+1},
$$

de lo que puede verse que este factor toma valores comprendidos entre 1 y 2 . Además, el caso general para mesones escalares es fácil de derivar. Notemos que poniendo $D=2 \Delta+3$ y $n=0$ recuperamos la expresión para el modelo de $D 3 D 7$-branas. Por otro lado, los factores para los modelos de $D 4 D 6 \overline{D 6}$ y $D 4 D 8 \overline{D 8}$-branas son $\frac{16(\Delta+1)}{8 \Delta+11}$ y $\frac{4(4 \Delta+3)}{8 \Delta+9}$ respectivamente.

\section{5. $x$ exponencialmente pequeño}

Como era de esperarse, la inclusión de cuerdas excitadas da lugar a un nuevo término en las funciones de estructura que domina para $x$ pequeño. Este tiene un efecto interesante sobre los momentos $M_{n}^{(1,2)}$. Para $n>2$, la nueva contribución converge y da la misma dependencia $q^{2-2 \Delta}$ encontrada en el cálculo de SUGRA, correspondiente a los operadores de doble traza encontrados en la Sección 4.4. Sin embargo, la suma de momentos para $n=2$ diverge ahora al tender $x \rightarrow 0$. Vemos con esto que la aproximación que estamos usando ha fallado en este régimen (región $\mathbf{C}$ ).

Estamos considerando solamente el efecto dominante en la expansión $\frac{1}{N}$, correspondiente a una sola cuerda. Si bien la dependencia en $x$ de las funciones de estructura $\left(\frac{N}{7.1 .67)}\right.$ es consistente con los cálculos de teoría de campos (7.1.1), la corrección de $O\left((\lambda)^{-1 / 2}\right)$ en el exponente es crucial para la convergencia de los momentos.

La forma de tener en cuenta esto es la siguiente. Al considerar la región $\mathbf{B}$, $\exp (-\sqrt{\lambda}) \ll$ $x \ll \frac{1}{\sqrt{\lambda}}$, despreciamos el factor de Regge $\tilde{s}^{\alpha^{\prime} t} / 2$. Este se vuelve importante para la región $\mathbf{C}, x \ll$ $\exp (-\sqrt{\lambda})$. Estudiaremos el efecto de este factor en el régimen $1 \ll \lambda \ll N$, y tomando valores exponencialmente grandes de la variable de Mandelstam $\tilde{s}$, de manera que $\frac{\log s}{\sqrt{\lambda}}$ se mantenga fijo.

Este factor no es trivial pues, en esta escala, la interacción no puede considerarse local en el espacio $A d S$. El parámetro $\tilde{t}$ es ahora un operador diferencial, por lo que debemos reemplazarlo 
por

$$
\alpha^{\prime} \tilde{t} \rightarrow \alpha^{\prime} \nabla^{2}=\alpha^{\prime}\left(\frac{R^{2} t}{r^{2}}+\nabla_{\perp}^{2}\right),
$$

pues aquí tomamos en cuenta la transferencia de momento en las direcciones transversales [5, 47, 3]. Entonces este factor de Regge se escribe

$$
m^{\alpha^{\prime} \tilde{t} / 2} \sim\left(\alpha^{\prime} \tilde{s}\right)^{\alpha^{\prime} \tilde{t} / 2} \sim x^{-\alpha^{\prime} \tilde{t} / 2} \sim x^{-\alpha^{\prime} \nabla^{2} / 2}
$$

dentro del prefactor. Al igual que en las referencias [5, 47, 3] el laplaciano transverso es proporcional a $\frac{1}{R^{2}}$. Con esto, el término a agregar es del orden de $\frac{1}{\sqrt{\lambda}}$.

En este régimen, consideramos que la interacción se produce por el intercambio de un pomerón en el canal $t$. Teniendo en cuenta la transferencia de momento transversal es posible obtener la corrección dominante al límite de acoplamiento fuerte para la ordenada al origen $\alpha_{0}=2$. Veremos que $\tilde{s}^{\alpha^{\prime} \tilde{t} / 2}$ resultará ser un operador de difusión.

Vamos a diagonalizar ahora el operador diferencial 7.5.1), para obtener los exponentes de Regge. El operador laplaciano escalar, al actuar sobre un campo de espín $j$-denotado genéricamente $\Phi_{j}$ - viene dado por

$$
\nabla_{0}^{2} \Phi_{j}=\frac{1}{\sqrt{-g}} \partial_{M}\left[\sqrt{-g} g^{M N} \partial_{N} \Phi_{j}\right]
$$

donde $g_{M N}$ es la métrica en diez dimensiones. Dado el espacio curvo $A d S_{5} \times W^{5}$, con $W^{5}$ una variedad compacta de Einstein, su métrica se escribe

$$
d s^{2}=\frac{r^{2}}{R^{2}} \eta_{\mu \nu} d x^{\mu} d x^{\nu}+\frac{R^{2}}{r^{2}} d r^{2}+R^{2} d s_{W}^{2}
$$

Con esto, el operador diferencial es

$$
\alpha^{\prime} \nabla_{0}^{2}=\alpha^{\prime} \frac{R^{2}}{r^{2}} t+\frac{\alpha^{\prime}}{R^{2}}\left(r^{2} \partial_{r}^{2}+5 r \partial_{r}\right)+\alpha^{\prime} \nabla_{W}^{2}
$$

Realizamos ahora el cambio de variables $e^{u}=r / r_{0}$, con $r_{0}=\Lambda R^{2}$ el cutoff IR. El operador laplaciano covariante actuando sobre el campo $\Phi_{j}$ se define

$$
\alpha^{\prime} \hat{D}_{j}^{2} \Phi_{j}=\alpha^{\prime} e^{2 j u} \nabla_{0}^{2}\left[e^{-2 j u} \Phi_{j}\right] .
$$

En nuestro caso, para un gravitón de $j=2$ obtenemos la forma explícita de este lagrangiano covariante dada por

$$
\alpha^{\prime} \hat{D}^{2}=\frac{1}{\sqrt{\lambda}} t e^{-2 u} R^{2}+\frac{1}{\sqrt{\lambda}}\left(\partial_{u}^{2}-4\right)+\alpha^{\prime} \nabla_{W}^{2}
$$

donde omitimos el subíndice $j=2$ del operador $\hat{D}^{2}$. Luego, siguiendo [5] y [3, modificamos la ecuación 7.2.32 para incluir el efecto del operador de difusión $\tilde{s}^{\alpha^{\prime} \tilde{t} / 2} \sim x^{-\alpha^{\prime} t \tilde{t} / 2}$. Resulta la integral

$$
\begin{aligned}
& n_{\mu}^{*} n_{\nu} I m_{e x c} T^{\mu \nu}= \\
& \frac{\pi \alpha^{\prime}}{8} \sum_{m=1}^{\infty} \int d \Omega_{3} d r \sqrt{-g} v_{i} v^{i} F_{k p}^{*}(q) F_{n}^{p}(q)\left(\frac{\alpha^{\prime} \tilde{s}}{4}\right)^{\alpha^{\prime} \tilde{t} / 2}\left(\partial^{k} X^{*}(P) \partial^{n} X(P)\right) \delta\left(m-\frac{\alpha^{\prime} \tilde{s}}{4}\right) .
\end{aligned}
$$


La acción del operador $x^{-\alpha^{\prime} \tilde{t} / 2}$ sobre $\partial^{k} X^{*}(P) \partial^{n} X(P)$ viene dada esencialmente por $\hat{D}^{2}\left(\partial^{k} X^{*}(P) \partial^{n} X(P)\right)$, que es proporcional a $P^{k} P^{n} \hat{D}^{2}\left(X^{*}(P) X(P)\right)$. Con esto, la exponencial de $\hat{D}^{2}$ es no local, siendo su tiempo de difusión $\ln x$.

Cuando $x$ es exponencialmente pequeño, la estructura geométrica correspondiente a la región $r_{0}$ donde se rompe la simetría conforme se vuelve relevante ${ }^{29}$ En esta región seguimos utilizando las mismas expresiones para el tensor de campo $F^{m n}$ pues este no se ve afectado por la ruptura de simetría conforme mencionada, dado que caen exponencialmente en esta región ${ }^{30}$. Luego, obtenemos la expresión para las funciones de estructura

$$
\begin{aligned}
& F_{1}=\frac{\pi^{2} \alpha^{\prime} R^{2} q^{2}}{16 x^{2}} J_{1}^{D 3 D 7}, \\
& F_{2}=\frac{\pi^{2} \alpha^{\prime} R^{2} q^{2}}{8 x}\left(J_{0}^{D 3 D 7}+J_{1}^{D 3 D 7}\right),
\end{aligned}
$$

donde las integrales son

$$
J_{j}^{D 3 D 7}=\int d \omega \int d \Omega_{3} \sqrt{\hat{g}_{\Omega_{3}}} v^{i} v_{i} \omega^{3} K_{j}^{2}(\omega)\left(\frac{\alpha^{\prime} \tilde{s}}{4}\right)^{\alpha^{\prime} \tilde{t} / 2}\left(X^{*} X\right) .
$$

Consideramos ahora el límite para $x$ exponencialmente pequeño. Podemos reemplazar ahora el operador diferencial $-\nabla^{2}=-D^{2}$ por su menor autovalor $\zeta$ en $(7.5 .2)$. Entonces se recupera el comportamiento esperado para las funciones de estructura pues $F_{1} \propto x^{-2+\alpha^{\prime} \zeta / 2}$ y $F_{2} \propto$ $x^{-1+\alpha^{\prime} \zeta / 2}$, donde la ordenada al origen del pomerón es $\alpha_{0}=2-\frac{\alpha^{\prime} \zeta}{2}$.

Para resolver las integrales $J_{j}^{D 3 D 7}$ tenemos que estudiar la caída de la menor autofunción de los operadores $\nabla_{0}^{2}$ (que es $r^{-4}$ ) y $\nabla^{2}$ (que es $r^{-2}$ ). Con esto, las integrales están dominadas por la región $\omega \approx 1$, resultando

$$
J_{j}^{D 3 D 7} \propto \int d \omega \int d \Omega_{3} \sqrt{\hat{g}_{\Omega_{3}}} v^{i} v_{i} \omega^{3} K_{j}^{2}(\omega) r^{-2} .
$$

Finalmente obtenemo\$ 31

$$
\begin{aligned}
& F_{1}=\frac{\pi^{2}}{16 x^{2}} \rho_{3}\left|c_{i}\right|^{2}\left(\frac{\Lambda^{2}}{q^{2}}\right)^{\Delta-1} \frac{1}{\sqrt{4 \pi g_{c} N}} I_{1,2 \Delta+3} x^{\alpha^{\prime} \zeta / 2} \propto x^{-\left(2+\alpha^{\prime}|\zeta| / 2\right)} \\
& F_{2}=\frac{\pi^{2}}{8 x} \rho_{3}\left|c_{i}\right|^{2}\left(\frac{\Lambda^{2}}{q^{2}}\right)^{\Delta-1} \frac{1}{\sqrt{4 \pi g_{c} N}}\left(I_{0,2 \Delta+3}+I_{1,2 \Delta+3}\right) x^{\alpha^{\prime} \zeta / 2} \propto x^{-\left(1+\alpha^{\prime}|\zeta| / 2\right)}
\end{aligned}
$$

donde el autovalor del operador de difusión es $\zeta=\frac{4}{R^{2}}\left(1-\Delta^{2}\right) \leq 0$. Observamos aquí que las relaciones tipo Callan-Gross son las mismas que (7.2.57) en la región $B$,

$$
F_{2}=2 x F_{1}\left(1+\frac{I_{0}}{I_{1}}\right)=2 x F_{1} \frac{2 \Delta+3}{\Delta+2} .
$$

Todos los cálculos realizados en esta Sección corresponden a los mesones escalares en el modelo D3D7. Para mesones vectoriales en este modelo, los cálculos son similares. Las relaciones de tipo Callan-Gross son las mismas que las obtenidas en la región $\mathbf{B}$, discutidas en la Sección 7.3. La única diferencia será el valor del autovalor para el operador de difusión, aunque conceptualmente este factor resulta muy importante.

\footnotetext{
${ }^{29}$ Estamos pensando siempre en modelos duales gravitatorios de la forma $A d S_{5} \times W$, como el caso de D3D7 y $\mathcal{N}=1^{*}$. En los casos D4D6 $\overline{\mathrm{D} 6}$ y $\mathrm{D} 4 \mathrm{D} 8 \overline{\mathrm{D} 8}$ la métrica asintótica no es conforme.

${ }^{30}$ La forma asintótica de $K_{\alpha}(y)$ es $\sqrt{\pi /(2 y)} \exp (-y)$ para $y \gg\left|\alpha^{2}-1 / 4\right|$.

${ }^{31}$ Estas son las funciones de estructura para el modelo D3D7 en el caso escalar (y también vectorial) para la región C. Comparar con 7.2.55.
} 


\subsection{Discusión de los resultados}

En este Capítulo hemos estudiado el DIS de leptones cargados por hadrones de espín cero y uno a valores pequeños del parámetro $x$ de Bjorken (regiones $\mathbf{B}$ y $\mathbf{C}$ ) partiendo de amplitudes en teoría de cuerdas. Consideramos tanto mesones escalares como vectoriales con un solo sabor en el límite planar y de acoplamiento fuerte de la teoría de gauge $(N \gg \lambda \gg 1)$. Al igual que en el Capítulo anterior estudiamos este proceso para distintos modelos holográficos duales con D p-branas de sabor en fondos de teorías de supercuerdas tipo IIA y tipo IIB. Derivamos el tensor hadrónico, compuesto de las funciones de estructura, para mesones escalares y vectoriales polarizados, obteniendo de estos últimos una rica estructura tensorial de Lorentz, compuesta por ocho funciones de estructura.

Nuestro resultado más interesante es que encontramos nuevas relaciones de tipo Callan-Gross para varias de las funciones de estructura. Estas son formalmente similares para los diferentes modelos holográficos investigados. Esto podría interpretarse como una señal de comportamiento universal para teorías de gauge confinantes que posean un modelo dual en la teoría de cuerdas donde los mesones puedan interpretarse como fluctuaciones de las $\mathrm{D} p$-branas de sabor en la aproximación de prueba. Esta universalidad encontrada no se relaciona con la supersimetría ni con la simetría conforme de las teorías de gauge duales, pues estas difieren fuertemente respecto de aquellas simetrías. Las simetrías de interés son aquí las correspondientes a la acción de DiracBorn-Infeld, que es la que da la similitud de las soluciones entre los diversos modelos.

Específicamente, encontramos la relación tipo Callan-Gross $F_{2}=2 x F_{1}\left(1+\frac{I_{0}}{I_{1}}\right)$, donde $0 \leq$ $\frac{I_{0}}{I_{1}} \leq 1$ es una función de la dimensión de escaleo $\Delta$ del mesón vectorial en cuestión. Recordemos del Capítulo anterior que la relación obtenida para el régimen de supergravedad $x \lesssim 1$ era $F_{2}=$ $2 F_{1}$, por lo que recuperamos en este régimen el factor $x$ de la relación de Callan-Gross original, además del nuevo factor $\left(1+\frac{I_{0}}{I_{1}}\right)$. También encontramos las relaciones $b_{2}=2 x b_{1}\left(1+\frac{I_{0}}{I_{1}}\right)$, $b_{1}=3 F_{1}, b_{4}=-\frac{1}{2} b_{3}$ y $g_{1}=0$. Estas son distintas a las obtenidas en el Capítulo anterior para la región $\mathbf{A}$, régimen de supergravedad.

Para la región $\mathbf{C}$, correspondiente a $x$ exponencialmente pequeño, en la que el tamaño de las cuerdas se vuelve del orden de la escala del espacio $A d S$, la interacción se vuelve no local y debe tomarse en cuenta el crecimiento de las cuerdas a través de la introducción de un operador de difusión. En la región $\mathbf{D}$, el grupo de renormalización de la hoja de mundo debe usarse para incluir el efecto del crecimiento de las cuerdas. El análisis hecho hasta aquí, correspondiente al límite planar de la teoría de gauge fuertemente acoplada corresponde a la dispersión de un leptón por un hadrón entero. Esperaríamos que esta región $\mathbf{D}$, a la que no tenemos acceso con estos cálculos, nos revelara la estructura partónica subyacente, con la que recuperaríamos el crecimiento de las funciones de estructura con $q^{2}$, como muestra la fenomenología. Nuestra aproximación falla en esta región pues para valores del tamaño de la cuerda comparables con el radio del $A d S$ no puede tomarse la amplitud de dispersión para cuerdas en el espacio plano y multiplicarla por la raíz de la métrica para construir la amplitud de dispersión en espacio curvo, como se explicó en la Sección 3.2 .

Hemos respondido varias preguntas. Por un lado, obtuvimos las ocho funciones de estructura para el caso de mesones vectoriales e investigamos su dependencia en $x$ tanto para las regiones B como C, en el límite planar de teorías de gauge fuertemente acopladas. La dependencia de las funciones de estructura con $q^{2}$ obtenida en estas regiones, tanto para el caso de mesones escalares como el de vectoriales, es el esperado de consideraciones de OPE para el caso del límite planar en acoplamiento fuerte. Al igual que en el caso de glueballs estudiado en [5] y descripto en la Sección 7.1 obtenemos una ley de potencias. Por otro lado, de haber considerado colisión de cuerdas en espacio plano habríamos obtenido un comportamiento exponencial, indicando 
una amplitud muy suave, pues no habría partones en aquel caso caso. En el caso estudiado de espacio curvo, este comportamiento se explica pues la tensión de la cuerda vista por un observador inercial en el borde aumenta al ir del interior al borde del espacio, y con esto su tamaño se vuelve más pequeño, del orden de la inversa del momento transferido.

En términos de la teoría en el interior, cuando $x \sim \frac{1}{\sqrt{\lambda}}$ la energía para un observador en el interior se hace del orden de la masa de la cuerda, $\tilde{s} \sim \frac{1}{\alpha^{\prime}}$. Por lo tanto, en esta región de parámetros la dinámica de las cuerdas en el interior se vuelve importante. Notemos que también en QCD el intercambio de pomerón domina la estructura de gluones para el régimen $s \approx \frac{q^{2}}{x}$ para $x$ pequeño. La contribución del pomerón a las PDF es, como dijimos en la Sección 7.1. proporcional a $x^{-\alpha_{0}}$, donde $\alpha_{0}$ es la ordenada al origen de la trayectoria del pomerón. De aquí que se espere el comportamiento de la forma $F_{1} \propto x^{-2+\mathcal{O}(1 / \sqrt{\lambda})}$ y $F_{2} \propto x^{-1+\mathcal{O}(1 / \sqrt{\lambda})}$ que encontramos. 


\section{Capítulo 8}

\section{Secciones eficaces diferenciales}

En este Capítulo estudiamos las secciones eficaces diferenciales para DIS de leptones cargados por hadrones en el caso general para espín $0, \frac{1}{2}$ y 1 , centrándonos en este último caso. Luego aplicamos nuestros resultados para las funciones de estructura obtenidos con métodos holográficos en los Capítulos 6 y 7, correspondientes a las regiones A, B y C. Los resultados de este Capítulo se encuentran en nuestro trabajo [4].

En la Sección 8.1 realizamos algunas definiciones complementarias a las vistas en el Capítulo 4 pertinentes al estudio de las secciones eficaces. En la Sección 8.2 estudiamos el caso general de DIS de leptones por mesones de espín 1, estudiando las distintas funciones de estructura según las polarizaciones de cada partícula interviniente. En la Sección 8.3 introducimos nuestros resultados provenientes de modelos holográficos duales al cálculo de las secciones eficaces para el régimen de acoplamiento fuerte y $N$ grande $1 \ll \lambda \ll N N^{1}$ Finalmente, en la Sección 8.4 discutiremos los resultados obtenidos.

\subsection{Generalidades}

Utilizaremos aquí la notación y convenciones definidas en la Sección 4 . Recordemos que la sección eficaz diferencial de DIS puede escribirse

$$
\frac{d^{2} \sigma}{d x d y d \phi}=\frac{e^{4}}{16 \pi^{2} q^{4}} y l^{\mu \nu} W_{\mu \nu},
$$

donde el tensor leptónico $l^{\mu \nu}$ depende del haz incidente y puede derivarse fácilmente de QED. Este puede separarse en su parte simétrica y antisimétrica

$$
\begin{aligned}
l_{\frac{1}{2}}^{\mu \nu} & =\left[2\left(k^{\mu} k^{\prime \nu}+k^{\nu} k^{\prime \mu}\right)-2 \eta^{\mu \nu} k \cdot k^{\prime}\right]+\left[-2 i \epsilon^{\mu \nu \alpha \beta} q_{\alpha} s_{l \beta}\right] \\
& =l_{\text {sym }}^{\mu \nu}+l_{\text {ant }}^{\mu \nu}
\end{aligned}
$$

donde asumimos leptones no masivos, $m_{l}^{2} \sim 0$ y $s_{l \beta}$ es el espín del leptón.

Por otro lado, el tensor hadrónico $W_{\mu \nu}$ describe la estructura interna del hadrón y no puede ser calculado perturbativamente desde QCD. En general, para hadrones de espín $s=1, \frac{1}{2}$ y 0

\footnotetext{
${ }^{1}$ Recordemos que esta es la expresión correcta para el caso D3D7, correspondiente a supergravedad de tipo IIB, mientras que para los otros modelos estudiados, a saber D4D8 $\overline{\mathrm{D} 8}$ y $\mathrm{D} 4 \mathrm{D} 6 \overline{\mathrm{D} 6}$, correspondientes a supergravedad del tipo IIA, la relación correcta es $1 \ll \lambda U \ll N^{\frac{4}{3}}$, donde $U$ es la coordenada radial. Ver nota 2 del Capítulo 1.
} 
podemos escribir respectivamente

$$
\begin{aligned}
& W_{\mu \nu}^{1}=W_{\mu \nu}^{S F}+W_{\mu \nu}^{S b}+W_{\mu \nu}^{A g} \\
& W_{\mu \nu}^{\frac{1}{2}}=W_{\mu \nu}^{S F}+W_{\mu \nu}^{A g} \\
& W_{\mu \nu}^{0}=W_{\mu \nu}^{S F}
\end{aligned}
$$

donde

$$
\begin{aligned}
W_{\mu \nu}^{S F} & =F_{1} \eta_{\mu \nu}-\frac{F_{2}}{P \cdot q} P_{\mu} P_{\nu}, \\
W_{\mu \nu}^{S b} & =b_{1} r_{\mu \nu}-\frac{b_{2}}{6}\left(s_{\mu \nu}+t_{\mu \nu}+u_{\mu \nu}\right)-\frac{b_{3}}{2}\left(s_{\mu \nu}-u_{\mu \nu}\right)-\frac{b_{4}}{2}\left(s_{\mu \nu}-t_{\mu \nu}\right), \\
W_{\mu \nu}^{A g} & =-\frac{i g_{1}}{P \cdot q} \epsilon_{\mu \nu \lambda \sigma} q^{\lambda} s^{\sigma}-\frac{i g_{2}}{(P \cdot q)^{2}} \epsilon_{\mu \nu \lambda \sigma} q^{\lambda}\left(P \cdot q s^{\sigma}-s \cdot q P^{\sigma}\right) .
\end{aligned}
$$

Los supraíndices no indican más que las propiedades de simetría de su estructura tensorial, siendo $S$ para los términos simétricos y $A$ para los asimétricos. Los tensores $r_{\mu \nu}, s_{\mu \nu}, t_{\mu \nu}$ y $u_{\mu \nu}$ están definidos en la Sección 4. Recordemos que 55]

$$
\begin{aligned}
& s^{\sigma} \equiv \frac{-i}{M^{2}} \epsilon^{\sigma \alpha \beta \rho} \zeta_{\alpha}^{*} \zeta_{\beta} P_{\rho}, \\
& \kappa=1-4 x^{2} t,
\end{aligned}
$$

donde $\zeta^{\mu}$ es el vector polarización del hadrón. Al multiplicar el tensor hadrónico y el leptónico solo quedarán los productos con ambos factores simétricos o ambos antisimétricos, resultando la sección eficaz

$$
\frac{d^{2} \sigma}{d x d y d \phi}=\frac{e^{4}}{16 \pi^{2} q^{4}} y\left[l_{s y m}^{\mu \nu} W_{\mu \nu}^{S F}+l_{a n t}^{\mu \nu} W_{\mu \nu}^{A g}+l_{s y m}^{\mu \nu} W_{\mu \nu}^{S b}\right] .
$$

Los hadrones de espín cero solo tendrán el primer término. Los de espín $\frac{1}{2}$ tendrán los dos primeros términos, y los de espín 1 polarizados presentarán los tres.

\subsection{Sección eficaz de DIS y funciones de estructura: Caso general}

En esta Sección derivaremos las secciones eficaces diferenciales de DIS para hadrones con espín $s=0, \frac{1}{2}$ y 1 . En primer lugar consideraremos el caso general y luego estudiaremos diferentes estados de polarización de los hadrones.

\subsubsection{Polarización arbitraria}

Comenzaremos con el primer término, que está presente tanto en los hadrones de espín 0 como $\frac{1}{2}$ y 1 . Obtenemos, en términos de las funciones de estructura $F_{1}$ y $F_{2}$,

$$
\begin{aligned}
\frac{e^{4}}{16 \pi^{2} q^{4}} \text { y } l_{s y m}^{\mu \nu} W_{\mu \nu}^{S F} & =\frac{e^{4} M E}{4 \pi^{2} q^{4}}\left\{x y^{2} F_{1}+\left[1-y+x^{2} y^{2} t\right] F_{2}\right\} \\
& \simeq \frac{e^{4} M E}{4 \pi^{2} q^{4}}\left\{x y^{2} F_{1}+[1-y] F_{2}\right\}
\end{aligned}
$$


donde hemos aproximado $|t| \ll 1$ en el último paso $2^{2}$

El segundo término, que aparece tanto para espín $s=\frac{1}{2}$ como para $s=1$ es

$$
\begin{aligned}
\frac{e^{4}}{16 \pi^{2} q^{4}} \text { y } l_{a n t}^{\mu \nu} W_{\mu \nu}^{A g}= & \frac{e^{4} M E}{4 \pi^{2} q^{4}}\left\{y^{2} g_{1}\left[2 x \frac{\left(s_{h} \cdot s_{l}\right)}{(p \cdot q)}+\frac{\left(q \cdot s_{h}\right)\left(q \cdot s_{l}\right)}{(p \cdot q)^{2}}\right]\right. \\
& \left.+2 x y^{2} g_{2}\left[\frac{\left(s_{h} \cdot s_{l}\right)}{(p \cdot q)}-\frac{\left(q \cdot s_{h}\right)\left(p \cdot s_{l}\right)}{(p \cdot q)^{2}}\right]\right\} .
\end{aligned}
$$

Podemos ver que este término depende exclusivamente de las funciones de estructura $g_{1}$ y $g_{2}$. Notemos que en la expresión para el primer término solamente contraemos factores simétricos, mientras que en la expresión para el segundo término son solo factores antisimétricos. Además, en el último cálculo utilizamos la identidad $\epsilon^{\mu \nu \alpha \beta} \epsilon_{\mu \nu \lambda \sigma}=-2\left(\eta_{\lambda}^{\alpha} \eta_{\sigma}^{\beta}-\eta_{\sigma}^{\alpha} \eta_{\lambda}^{\beta}\right)$.

Calculamos ahora la forma más general del tercer término, que solo aparece para hadrones de espín 1, y puede escribirse en términos de las funciones de estructura $b_{i}$. Esto no ha sido calculado antes hasta donde sabemos. En primer lugar calculamos las contracciones

$$
l_{s y m}^{\mu \nu} r_{\mu \nu} \quad ; \quad l_{s y m}^{\mu \nu} s_{\mu \nu} \quad ; \quad l_{s y m}^{\mu \nu} t_{\mu \nu} \quad ; \quad l_{s y m}^{\mu \nu} u_{\mu \nu} .
$$

Para simplificar la notación definimos el pseudoproducto escalar $\langle\cdot \cdot\rangle$ como

$$
\left\langle k_{1} k_{2}\right\rangle \equiv \frac{3}{2} \frac{\left(k_{1} \cdot \zeta^{*}\right)\left(k_{2} \cdot \zeta\right)+c . c .}{(P \cdot q)^{2}},
$$

donde c.c. indica complejo conjugado. Este producto es linear en ambas componentes y conmutativo. Además, dado que tomamos el límite $|t| \ll 1$ tenemos $\kappa=1$. Con esto obtenemos

$$
\begin{aligned}
& l_{s y m}^{\mu \nu} r_{\mu \nu}= {\left[2\left(k^{\mu} k^{\prime \nu}+k^{\nu} k^{\prime \mu}\right)-2 \eta^{\mu \nu} k \cdot k^{\prime}\right] \frac{1}{(P \cdot q)^{2}}\left(q \cdot \zeta^{*} q \cdot \zeta-\frac{1}{3}(P \cdot q)^{2}\right) \eta_{\mu \nu} } \\
&=-\frac{4}{3}\left(k \cdot k^{\prime}\right)[\langle q q\rangle-1] \\
&= \frac{4}{3} M E x y[\langle q q\rangle-1], \\
& l_{s y m}^{\mu \nu} s_{\mu \nu}= {\left[2\left(k^{\mu} k^{\prime \nu}+k^{\nu} k^{\prime \mu}\right)-2 \eta^{\mu \nu} k \cdot k^{\prime}\right] \frac{2}{(P \cdot q)^{3}}\left(q \cdot \zeta^{*} q \cdot \zeta-\frac{1}{3}(P \cdot q)^{2}\right) P_{\mu} P_{\nu} } \\
&= \frac{4}{3}\left[\frac{2(P \cdot k)\left(P \cdot k^{\prime}\right)}{(P \cdot q)}-\frac{P^{2}\left(k \cdot k^{\prime}\right)}{(P \cdot q)}\right][\langle q q\rangle-1] \\
&=-\frac{8}{3} M E[\langle q q\rangle-1]\left[\frac{1}{y}-1+x^{2} y t\right], \\
& l_{s y m}^{\mu \nu} t_{\mu \nu} \quad=\left[2\left(k^{\mu} k^{\prime \nu}+k^{\nu} k^{\prime \mu}\right)-2 \eta^{\mu \nu} k \cdot k^{\prime}\right] \frac{1}{2(P \cdot q)^{2}} \times \\
&=\frac{-1}{\langle q q\rangle-1} l_{s y m}^{\mu \nu} s_{\mu \nu}+\frac{4}{3}\left\langle q k^{\prime}\right\rangle(k \cdot P)+\frac{4}{3}\langle q k\rangle\left(k^{\prime} \cdot P\right) \\
&=\frac{4}{3} M E\left[2\left(\frac{1}{y}-1+x^{2} y t\right)-\left\langle q k^{\prime}\right\rangle-\langle q k\rangle(1-y)\right]
\end{aligned}
$$

\footnotetext{
${ }^{2}$ Ecuación (5.2) de [50]. El factor extra $2 \pi$ en el denominador aparece porque aquí calculamos $\frac{d^{2} \sigma}{d x d y d \phi}$ como en [50]. Por otro lado, en la referencia [55] se calcula $\frac{d^{2} \sigma}{d x d y}=2 \pi \frac{d^{2} \sigma}{d x d y d \phi}$.
} 
y

$$
\begin{aligned}
l_{s y m}^{\mu \nu} u_{\mu \nu} & =\left[2\left(k^{\mu} k^{\prime \nu}+k^{\nu} k^{\prime \mu}\right)-2 \eta^{\mu \nu} k \cdot k^{\prime}\right] \frac{1}{(P \cdot q)}\left[\left(\zeta_{\mu}^{*} \zeta_{\nu}+\zeta_{\nu}^{*} \zeta_{\mu}\right)-\frac{2}{3} M^{2} \eta_{\mu \nu}-\frac{2}{3} P_{\mu} P_{\nu}\right] \\
& =\left[\frac{4}{3}\left\langle k k^{\prime}\right\rangle(P \cdot q)-2 \frac{M^{2}\left(k \cdot k^{\prime}\right)}{(P \cdot q)}\right]-\frac{2 M^{2}}{(P \cdot q)[\langle q q\rangle-1]} l_{s y m}^{\mu \nu} r_{\mu \nu}-\frac{1}{\langle q q\rangle-1} l_{s y m}^{\mu \nu} s_{\mu \nu} \\
& =\frac{8}{3} M E\left[-y\left\langle k k^{\prime}\right\rangle+\left(\frac{1}{y}-1\right)+2 x^{2} y t\right]
\end{aligned}
$$

donde $r_{\mu \nu}, s_{\mu \nu}, t_{\mu \nu}$ y $u_{\mu \nu}$ fueron definidos en la Sección 4.3 . Finalmente, escribiendo la contribución a la sección eficaz de las funciones $b_{i}$ como $\alpha_{1} b_{1}+\alpha_{2} b_{2}+\alpha_{3} b_{3}+\alpha_{4} b_{4}$ encontramos la expresión para los coeficientes $\alpha_{i}$

$$
\begin{aligned}
\alpha_{1}= & \frac{e^{4}}{16 \pi^{2} q^{2}} y l_{s y m}^{\mu \nu} r_{\mu \nu}=\frac{M E e^{4}}{2^{2} 3 \pi^{2} q^{4}} x y^{2}[\langle q q\rangle-1] \\
\alpha_{2}= & -\frac{e^{4}}{16 \pi^{2} q^{4}} \frac{y}{6} l_{s y m}^{\mu \nu}\left(s_{\mu \nu}+t_{\mu \nu}+u_{\mu \nu}\right) \\
= & \frac{M E e^{4}}{2^{3} 3^{2} \pi^{2} q^{4}} \times \\
& {\left[2(\langle q q\rangle-3)(1-y)+y\left\langle q k^{\prime}\right\rangle+y(1-y)\langle q k\rangle+2 y^{2}\left\langle k k^{\prime}\right\rangle-2(4-\langle q q\rangle) x^{2} y^{2} t\right] } \\
\alpha_{3}= & -\frac{e^{4}}{16 \pi^{2} q^{4}} \frac{y}{2} l_{s y m}^{\mu \nu}\left(s_{\mu \nu}-u_{\mu \nu}\right) \\
= & \frac{M E e^{4}}{2^{2} 3 \pi^{2} q^{4}}\left[(1-y)\langle q q\rangle-y^{2}\left\langle k k^{\prime}\right\rangle+x^{2} y^{2} t(\langle q q\rangle+1)\right] \\
\alpha_{4}= & -\frac{e^{4}}{16 \pi^{2} q^{4}} \frac{y}{2} l_{s y m}^{\mu \nu}\left(s_{\mu \nu}-t_{\mu \nu}\right) \\
= & -\frac{M E e^{4}}{2^{3} 3 \pi^{2} q^{4}}\left[2(1-y)\langle q q\rangle-y\left\langle q k^{\prime}\right\rangle-\langle q k\rangle y(1-y)+2\langle q q\rangle x^{2} y^{2} t\right]
\end{aligned}
$$

Utilizando la linealidad de $\langle\cdot \cdot\rangle$ obtenemos

$$
\begin{aligned}
& \frac{e^{4}}{16 \pi^{2} q^{4}} \text { y } l_{s y m}^{\mu \nu} W_{\mu \nu}^{S b}= \\
& \frac{M E e^{4}}{12 \pi^{2} q^{4}}\left\{b_{1} x y^{2}[\langle q q\rangle-1]+\frac{1}{6}\left[(2-3 y)\langle q q\rangle+y(2-3 y)\langle q k\rangle+2 y^{2}\langle k k\rangle-6(1-y)\right] b_{2}\right. \\
& \left.+\left[(1-y)\langle q q\rangle-y^{2}\langle k k\rangle+y^{2}\langle q k\rangle\right] b_{3}+\frac{1}{2}[(2-y)\langle q q\rangle+y(-2+y)\langle q k\rangle] b_{4} \cdot+\mathcal{O}(t)\right\} .
\end{aligned}
$$

Ahora bien, en el límite $\sqrt{-t} \ll 1$ podemos relacionar el valor de $\langle q q\rangle,\langle q k\rangle$ y $\langle k k\rangle$, pues en este límite es $\frac{q^{\mu}}{q^{0}}=\frac{k^{\mu}}{E}$, donde el índice de Lorentz $\mu$ va de 0 a 3 , y por ende en el sistema de referencia 
donde el hadrón está en reposo obtenemos las relaciones

$$
\begin{aligned}
\frac{q^{\mu} q^{\nu}}{M^{2}\left(q^{0}\right)^{2}} & =\frac{\frac{1}{2}\left(q^{\mu} k^{\nu}+k^{\mu} q^{\nu}\right)}{M^{2} q^{0} E}=\frac{k^{\mu} k^{\nu}}{M^{2} E^{2}} \\
2 \frac{q^{\mu} q^{\nu}}{(P \cdot q)^{2}} \zeta_{\mu} \zeta_{\nu}^{*} & =\frac{\left(q^{\mu} k^{\nu}+k^{\mu} q^{\nu}\right)}{(P \cdot q)(P \cdot k)} \zeta_{\mu} \zeta_{\nu}^{*}=2 \frac{k^{\mu} k^{\nu}}{(P \cdot k)^{2}} \zeta_{\mu} \zeta_{\nu}^{*} \\
\frac{3}{2} \frac{\left(q \cdot \zeta^{*}\right)(q \cdot \zeta)+c . c .}{(P \cdot q)^{2}} & =\frac{(P \cdot q)}{(P \cdot k)} \frac{3}{2} \frac{\left(q \cdot \zeta^{*}\right)(k \cdot \zeta)+c . c .}{(P \cdot q)^{2}}=\frac{(P \cdot q)^{2}}{(P \cdot k)^{2}} \frac{3}{2} \frac{\left(k \cdot \zeta^{*}\right)(k \cdot \zeta)+c . c .}{(P \cdot q)^{2}} \\
\langle q q\rangle & =\quad y\langle q k\rangle \quad=y^{2}\langle k k\rangle .
\end{aligned}
$$

Por lo tanto, para $\sqrt{-t} \ll 1$ la parte de la sección eficaz asociada con las funciones de estructura $b_{i}$ del tensor hadrónico es

$$
\frac{e^{4}}{16 \pi^{2} q^{4}} \text { y } l_{s y m}^{\mu \nu} W_{\mu \nu}^{S b}=\frac{M E e^{4}}{2^{2} 3 \pi^{2} q^{4}}(\langle q q\rangle-1)\left[b_{1} x y^{2}+b_{2}(1-y)\right]+\mathcal{O}(\sqrt{-t}) .
$$

Notar que en este límite las contribuciones a la sección eficaz provenientes de $b_{3}$ y $b_{4}$ son subdominantes en $t$ aun cuando estas funciones no lo son. Con esto, la sección eficaz diferencial para DIS de hadrones de espín 1 a este orden es

$$
\begin{aligned}
\frac{d \sigma_{\text {spin } 1}}{d x d y d \phi}= & \frac{M E e^{4}}{4 \pi^{2} q^{4}}\left\{\left[x y^{2} F_{1}+(1-y) F_{2}\right]+\frac{1}{3}(\langle q q\rangle-1)\left[x y^{2} b_{1}+(1-y) b_{2}\right]\right. \\
& \left.+y^{2} g_{1}\left[2 x \frac{\left(s_{h} \cdot s_{l}\right)}{(p \cdot q)}+\frac{\left(q \cdot s_{h}\right)\left(q \cdot s_{l}\right)}{(p \cdot q)^{2}}\right]+2 x y^{2} g_{2}\left[\frac{\left(s_{h} \cdot s_{l}\right)}{(p \cdot q)}-\frac{\left(q \cdot s_{h}\right)\left(p \cdot s_{l}\right)}{(p \cdot q)^{2}}\right]\right\} \\
= & \frac{M E e^{4}}{4 \pi^{2} q^{4}}\left\{x y^{2}\left[F_{1}+\frac{1}{3}(\langle q q\rangle-1) b_{1}\right]+(1-y)\left[F_{2}+\frac{1}{3}(\langle q q\rangle-1) b_{2}\right]\right. \\
& \left.+y^{2} g_{1}\left[2 x \frac{\left(s_{h} \cdot s_{l}\right)}{(p \cdot q)}+\frac{\left(q \cdot s_{h}\right)\left(q \cdot s_{l}\right)}{(p \cdot q)^{2}}\right]+2 x y^{2} g_{2}\left[\frac{\left(s_{h} \cdot s_{l}\right)}{(p \cdot q)}-\frac{\left(q \cdot s_{h}\right)\left(p \cdot s_{l}\right)}{(p \cdot q)^{2}}\right]\right\} .
\end{aligned}
$$

\subsubsection{Estudio de las polarizaciones del hadrón}

Durante toda esta Subsección nos referiremos al caso de espín 1. Calculamos ahora el factor adimensional

$$
\langle q q\rangle=\frac{3}{2} \frac{\left(q \cdot \zeta^{*}\right)(q \cdot \zeta)+c . c .}{(P \cdot q)^{2}}=3 \frac{q^{\mu} q^{\nu}}{(P \cdot q)^{2}} \zeta_{\mu} \zeta_{\nu}^{*}
$$

para distintas polarizaciones del hadrón a fin de obtener la sección eficaz diferencial de dispersión para DIS $\frac{d \sigma}{d x d y d \phi}$ para los casos particulares de interés fenomenológico, a saber: haz hadrónico no polarizado, longitudinalmente polarizado y transversalmente polarizado. También estudiamos polarizaciones parciales del haz hadrónico. En todos los casos el eje longitudinal o $\hat{z}$ se define con la componente espacial de $k^{\mu}$, correspondiente al haz leptónico incidente.

\section{Haz hadrónico no polarizado}

Para describir un haz hadrónico no polarizado debemos promediar sobre las polarizaciones obteniendo

$$
\overline{\zeta_{\mu} \zeta_{\nu}^{*}}=\frac{1}{3}\left(\eta_{\mu \nu} M^{2}+P_{\mu} P_{\nu}\right)
$$

Con esto,

$$
\langle q q\rangle_{\text {unpol }}=\langle\overline{q q}\rangle=\frac{1}{(P \cdot q)^{2}}\left[M^{2} q^{2}+(P \cdot q)^{2}\right]=1-4 x^{2} t=\kappa \simeq 1
$$


por lo que obtenemos una contribución nula por parte de las funciones de estructura $b_{i}$, como era de esperarse para un blanco no polarizado [55]. En particular, si consideramos el haz leptónico longitudinalmente polarizado, es $s_{l}=H_{l} k$ con $H_{l}= \pm 1$ para helicidad positiva o negativa respectivamente. Con esto resulta

$$
\frac{d \sigma}{d x d y d \phi}=\frac{M E e^{4}}{4 \pi^{2} q^{4}}\left\{x y^{2} F_{1}+(1-y) F_{2}\right\}
$$

\section{Polarización longitudinal}

Consideramos ahora el caso en el que el haz hadrónico está polarizado en la dirección del haz incidente de leptones, definido como eje $\hat{z}$. Tomamos entonces la polarización

$$
\zeta_{L}=\frac{M}{\sqrt{2}}\left(0,1, i H_{h}, 0\right) \quad \rightarrow \quad s^{\sigma}=H_{h} M \hat{z},
$$

donde $H_{h}= \pm 1$ indica que la polarización del hadrón es paralela o antiparalela al haz leptónico respectivamente. Entonces

$$
\begin{aligned}
\left(q \cdot \zeta_{L}\right) & =-\frac{M E^{\prime} \sin \theta}{\sqrt{2}} e^{i H_{h} \phi}, \\
\left(q \cdot \zeta_{L}^{*}\right)\left(q \cdot \zeta_{L}\right) & =\frac{M^{2} E^{\prime 2} \sin ^{2} \theta}{2}=\frac{q^{2} M^{2}}{2}\left[(1-y)+t x^{2} y^{2}\right], \\
\langle q q\rangle_{L P} & =\frac{3}{2} \frac{1}{(P \cdot q)^{2}} M^{2} E^{\prime 2} \sin ^{2} \theta=-6 x^{2} t\left[(1-y)+t x^{2} y^{2}\right] \simeq 0,
\end{aligned}
$$

En este caso, el factor $\langle q q\rangle-1$ solo aporta un signo negativo. En particular, para el haz leptónico longitudinalmente polarizado, es

$$
\frac{d \sigma}{d x d y d \phi}=\frac{M E e^{4}}{4 \pi^{2} q^{4}}\left\{x y^{2}\left[F_{1}-\frac{1}{3} b_{1}\right]+(1-y)\left[F_{2}-\frac{1}{3} b_{2}\right]-H_{l} H_{h}(2-y) y x g_{1}+\mathcal{O}(t)\right\} .
$$

\section{Polarización transversal}

Como el sistema en consideración tiene simetría acimutal podemos elegir cualquier polarización para el hadrón en el plano $(\hat{x}, \hat{y})$. Elegimos el hadrón polarizado en el eje $\hat{x}$ e imponemos

Con esto,

$$
\zeta_{T}=\frac{M}{\sqrt{2}}\left(0,0,1, i H_{h}\right) \quad \rightarrow \quad s^{\sigma}=H_{h} M \hat{x} \quad ; \quad H_{h}= \pm 1
$$

$$
\begin{aligned}
\left(q \cdot \zeta_{T}\right) & =\frac{M^{2}}{\sqrt{2}}\left[\sqrt{\frac{(1-y)}{-t}-x^{2} y^{2}} \sin \phi+i H_{h}\left(-\frac{1}{2 x t}+x y\right)\right] \\
& \simeq \frac{M^{2}}{\sqrt{2}}\left[\sqrt{\frac{(1-y)}{-t}} \sin \phi-\frac{i H_{h}}{2 x t}\right], \\
\left(q \cdot \zeta_{T}^{*}\right)\left(q \cdot \zeta_{T}\right) & =\frac{M^{4}}{2 t^{2}}\left[\frac{1}{4 x^{2}}-t(1-y) \sin ^{2} \phi\right], \\
\langle q q\rangle_{T P} & =\frac{3}{2} \frac{1}{(P \cdot q)^{2}} \frac{M^{4}}{t^{2}}\left[\frac{1}{4 x^{2}}-t(1-y) \sin ^{2} \phi\right] \\
& =6 x^{2}\left[\frac{1}{4 x^{2}}-t(1-y) \sin ^{2} \phi\right] \simeq \frac{3}{2},
\end{aligned}
$$


y el factor adimensional resulta $\langle q q\rangle_{T P}-1 \approx \frac{1}{2}$. En particular, para el haz leptónico longitudinalmente polarizado, es

$$
\begin{aligned}
\frac{d \sigma}{d x d y d \phi}= & \frac{M E e^{4}}{4 \pi^{2} q^{4}}\left\{x y^{2}\left[F_{1}+\frac{1}{6} b_{1}\right]+(1-y)\left[F_{2}+\frac{1}{6} b_{2}\right]\right. \\
& \left.+H_{l} H_{h} 2 x^{2} \sqrt{-t} \sqrt{1-y}\left(y g_{1}+2 g_{2}\right) \cos \phi\right\}
\end{aligned}
$$

\section{Polarizaciones parciales}

Los términos asociados a las funciones de estructura $b_{i}$ en (8.2.15), y en particular el factor adimensional $\langle q q\rangle-1$ pueden escribirse en una forma más general

$$
\frac{e^{4}}{16 \pi^{2} q^{4}} \text { y } l_{s y m}^{\mu \nu} W_{\mu \nu}^{S b}=\frac{M E e^{4}}{2 \sqrt{3} \pi^{2} q^{4}} \operatorname{Tr}\left(\rho \cdot \lambda_{8}\right)\left[b_{1} x y^{2}+b_{2}(1-y)\right]+\mathcal{O}(\sqrt{-t}) .
$$

donde $\lambda_{8}=\frac{1}{\sqrt{3}} \operatorname{diag}(1,1,-2)$ y $\rho$ es la matriz densidad para espín uno que refleja la posibilidad de tener una mezcla estadística de polarizaciones. Notemos que

$$
\operatorname{Tr}\left(\rho \cdot \lambda_{8}\right)=\frac{1}{2 \sqrt{3}}\left[\left\langle S_{x}^{2}\right\rangle+\left\langle S_{y}^{2}\right\rangle-2\left\langle S_{z}^{2}\right\rangle+3\left\langle S_{z}\right\rangle\right] .
$$

Podemos estudiar casos particulares con polarizaciones parciales longitudinales y transversales. En la base correspondiente al eje $\hat{z}$ tenemos

$$
\begin{aligned}
\rho_{p_{L P}} & =\left(\begin{array}{ccc}
\frac{H_{L}^{2}}{2} & 0 & 0 \\
0 & 1-H_{L}^{2} & 0 \\
0 & 0 & \frac{H_{L}^{2}}{2}
\end{array}\right) ; \\
\rho_{p_{T P}} & =\frac{1}{4}\left(\begin{array}{ccc}
2-H_{T}^{2} & 0 & -2+3 H_{T}^{2} \\
0 & 2 H_{T}^{2} & 0 \\
-2+3 H_{T}^{2} & 0 & 2-H_{T}^{2}
\end{array}\right) ; \\
\rho_{\text {unpol }} & =\frac{1}{3}\left(\begin{array}{lll}
1 & 0 & 0 \\
0 & 1 & 0 \\
0 & 0 & 1
\end{array}\right)
\end{aligned}
$$

donde $H_{L}$ y $H_{T}$ son las fracciones de polarización longitudinal y transversal del hadrón respectivamente. $]^{3}$ Aquí $H^{2}=1$ representa el caso totalmente polarizado y $H^{2}=\frac{2}{3}$ describe un haz hadrónico no polarizado. Con la notación anterior identificamos

$$
\begin{aligned}
\langle q q\rangle_{p L P} & =-3\left(\frac{2}{3}-H_{L}^{2}\right)\langle q q\rangle_{L P}+3\left(1-H_{L}^{2}\right)\langle q q\rangle_{\text {unpol }}, \\
\langle q q\rangle_{p T P} & =-3\left(\frac{2}{3}-H_{T}^{2}\right)\langle q q\rangle_{T P}+3\left(1-H_{T}^{2}\right)\langle q q\rangle_{\text {unpol }},
\end{aligned}
$$

donde el subíndice $p$ denota polarización parcial. El resultado final para la sección eficaz diferencial de DIS con hadrones parcialmente polarizados es la generalización de los casos anteriores. Para polarización parcial longitudinal $H_{L}$ es

$$
\begin{aligned}
\frac{d \sigma}{d x d y d \phi}= & \frac{M E e^{4}}{4 \pi^{2} q^{4}}\left\{x y^{2}\left[F_{1}+\left(\frac{2}{3}-H_{L}^{2}\right) b_{1}\right]+(1-y)\left[F_{2}+\left(\frac{2}{3}-H_{L}^{2}\right) b_{2}\right]\right. \\
& \left.-H_{l} H_{h}(2-y) y x g_{1}\right\}
\end{aligned}
$$

\footnotetext{
${ }^{3}$ Nuevamente, estamos escribiendo la polarización transversal en el eje $\hat{x}$, aunque elegirla en el eje $\hat{y}$ daría los mismos resultados.
} 
mientras que para polarización parcial transversal $H_{T}$ es

$$
\begin{aligned}
\frac{d \sigma}{d x d y d \phi}= & \frac{M E e^{4}}{4 \pi^{2} q^{4}}\left\{x y^{2}\left[F_{1}-\left(\frac{2}{3}-H_{T}^{2}\right) b_{1}\right]+(1-y)\left[F_{2}-\left(\frac{2}{3}-H_{T}^{2}\right) b_{2}\right]\right. \\
& \left.+H_{l} H_{h} 2 x^{2} \sqrt{-t} \sqrt{1-y}\left(y g_{1}+2 g_{2}\right) \cos \phi\right\} .
\end{aligned}
$$

Nuevamente, si se cumpliera $b_{2}(x)=2 x b_{1}(x)$ y tuviéramos leptones no polarizados, recuperaríamos las expresiones de [55].

\subsubsection{Amplitudes de helicidad}

Estudiaremos aquí las amplitudes de helicidad de la dispersión Compton asociada al proceso de DIS como se vio en el Capítulo 4. Notaremos $q$ al momento del fotón virtual y $p$ al momento del hadrón incidente como hasta ahora, mientras que $\lambda$ y $\lambda^{\prime}$ serán las helicidades inical y final respectivamente. La amplitud de la dispersión Compton es 4.2 .13

$$
\left(T_{\mu \nu}\right)_{\lambda \lambda^{\prime}} \equiv i \int d^{4} x e^{i q \cdot x}\left\langle p, \lambda^{\prime}\left|T\left(J_{\mu}(x) J_{\nu}(x)\right)\right| p, \lambda\right\rangle,
$$

cuya estructura tensorial es idéntica a la del tensor hadrónico $W_{\mu \nu}$. Como vimos en el Capítulo 4, el teorema óptico relaciona ambos tensores.

La amplitud de la dispersión Compton puede analizarse fácilmente para los diversos casos de helicidad de las partículas entrantes y salientes. Estas amplitudes se notan como $A_{h, H ; h^{\prime}, H^{\prime}}$, donde $h$ y $H$ denotan respectivamente las helicidades del fotón y del hadrón iniciales, mientras que las variables primadas denotan las helicidades de los estados finales respectivos. Para el caso de espín 1, todas estas helicidades pueden tomar los valores $-1,0 \mathrm{y}+1$. Asimismo, están restringidas a cumplir $h+H=h^{\prime}+H^{\prime}$ por conservación de la helicidad. Siguiendo a [50, 55], se calculan como

$$
A_{h H, h^{\prime} H^{\prime}}=\epsilon_{h}^{\mu \star} \epsilon_{h^{\prime}}^{\nu} T_{\mu \nu}\left(s_{H}\right)
$$

donde $\epsilon$ son los vectores polarización del fotón y $s_{H}$ es el espín del hadrón. En el caso de espín 1 , para un régimen cinemático general, y utilizando la notación de [55], donde $a_{3} \equiv \frac{b_{2}}{3}-b_{3} \mathrm{y}$ $a_{4} \equiv \frac{b_{2}}{3}-b_{4}$, estas se escriben

$$
\begin{aligned}
A_{++,++} & =F_{1}-\frac{\left(1-4 x^{2} t\right) b_{1}}{3}-\frac{x t}{3} a_{3}-g_{1}-4 x^{2} t g_{2} \\
& \simeq F_{1}-\frac{b_{1}}{3}-g_{1} \\
A_{+0,+0} & =F_{1}+\frac{2\left(1-4 x^{2} t\right) b_{1}}{3}+\frac{2 x t}{3} a_{3} \\
& \simeq F_{1}+\frac{2 b_{1}}{3} \\
A_{+0,0+} & =\sqrt{-t}\left[2 x\left(g_{1}+g_{2}\right)+\frac{a_{3}}{2}+\frac{\left(1-4 x^{2} t\right) a_{4}}{4}\right] \\
& \simeq \sqrt{-t}\left[2 x\left(g_{1}+g_{2}\right)+\frac{a_{3}}{2}+\frac{a_{4}}{4}\right] \\
A_{+-,+-} & =F_{1}-\frac{\left(1-4 x^{2} t\right) b_{1}}{3}-\frac{x t}{3} a_{3}+g_{1}-4 x^{2} t g_{2} \\
& \simeq F_{1}-\frac{b_{1}}{3}+g_{1}
\end{aligned}
$$




$$
\begin{aligned}
A_{+-, 00} & =\sqrt{-t}\left[2 x\left(g_{1}+g_{2}\right)-\frac{a_{3}}{2}-\frac{\left(1-4 x^{2} t\right) a_{4}}{4}\right] \\
\simeq & \sqrt{-t}\left[2 x\left(g_{1}+g_{2}\right)-\frac{a_{3}}{2}-\frac{a_{4}}{4}\right] \\
A_{+-,-+} & =-2 x t a_{3} \\
\simeq & 0 \\
A_{0+, 0+}= & -F_{1}+\frac{\left(1-4 x^{2} t\right) F_{2}}{2 x}+\frac{\left(1-4 x^{2} t\right) b_{1}}{3}-\frac{\left(3-12 x^{2} t+16 x^{4} t^{2}\right) b_{2}}{18 x} \\
& +\frac{4\left(1-2 x^{2} t\right) b_{3}}{3 x}+\frac{2 x t}{3}\left(1-4 x^{2} t\right) b_{4} \\
\simeq & -F_{1}+\frac{F_{2}}{2 x}+\frac{b_{1}}{3}-\frac{b_{2}}{6 x}+\frac{4 b_{3}}{3 x} \\
A_{00,00}= & -F_{1}+\frac{\left(1-4 x^{2} t\right) F_{2}}{2 x}-\frac{2\left(1-4 x^{2} t\right) b_{1}}{3}+\frac{\left(3-12 x^{2} t+16 x^{4} t^{2}\right) b_{2}}{9 x} \\
& -\frac{8\left(1-2 x^{2} t\right) b_{3}}{3 x}-\frac{4 x t}{3}\left(1-4 x^{2} t\right) b_{4} \\
\simeq & -F_{1}+\frac{F_{2}}{2 x}-\frac{2 b_{1}}{3}+\frac{b_{2}}{3 x}-\frac{8 b_{3}}{3 x} .
\end{aligned}
$$

En todos los casos la última línea corresponde a la aproximación para $|t| \ll 1$. Estas amplitudes son útiles para estudiar la estructura hadrónica en términos del DIS, especialmente para espines $s_{H}>\frac{1}{2}$. Proveen una forma sencilla para entender el significado de las funciones de estructura en términos teóricos, así como una idea experimental y fenomenológica de cómo pueden afectar a la amplitud de dispersión y qué experimentos realizar para medir sus distintas partes. Esto último se debe a que miden la contribución a la sección eficaz proveniente de una cierta polarización del proyectil y el blanco y se relacionan de manera lineal con las funciones de estructura.

\subsection{Sección eficaz y funciones de estructura: Resul- tados holográficos}

En los dos últimos Capítulos hemos obtenido el comportamiento dominante de las ocho funciones de estructura involucradas en el tensor hadrónico $W^{\mu \nu}$ para un blanco de espín 1, a saber $F_{1}, F_{2}, b_{1}, b_{2}, b_{3}, b_{4}, g_{1}$ y $g_{2}$, en términos de $x, \frac{\Lambda^{2}}{q^{2}}$ y algunos otros parámetros que definen el modelo holográfico dual del que se trate. El parámetro $x$ de Bjorken es extremadamente importante en nuestro análisis. El espacio de parámetros físico $0 \leq x \leq 1$ se separa en las cuatro regiones $\mathbf{A}, \mathbf{B}, \mathbf{C}$ y $\mathbf{D}$ definidos en la Introducción. En cada una de estas regiones las funciones de estructura tienen un comportamiento distinto. Estudiaremos la sección eficaz diferencial en las regiones A, B y $\mathbf{C}$, correspondientes a las funciones de estructura calculadas en los Capítulos anteriores. 


\subsubsection{Región A: $1 / \sqrt{\lambda} \ll x<1$}

Las funciones de estructura obtenidas en el Capítulo 6 son

$$
\begin{aligned}
F_{1} & =A^{S F}(x) \frac{1}{12 x^{3}}\left(1-x-2 x t-4 x^{2} t+4 x^{3} t+8 x^{3} t^{2}\right), \\
F_{2} & =A^{S F}(x) \frac{1}{6 x^{3}}\left(1-x+12 x t-14 x^{2} t-12 x^{2} t^{2}\right), \\
b_{1} & =A^{S F}(x) \frac{1}{4 x^{3}}(1-x-x t) \\
b_{2} & =A^{S F}(x) \frac{1}{2 x^{3}}\left(1-x-x^{2} t\right) \\
b_{3} & =A^{S F}(x) \frac{1}{24 x^{3}}\left(1-4 x+8 x^{2} t\right) \\
b_{4} & =A^{S F}(x) \frac{1}{12 x^{3}}\left(-1+4 x-2 x^{2} t\right) \\
g_{1} & =A^{S F}(x) \frac{t}{8 x^{2}}(-7+6 x+8 x t), \\
g_{2} & =A^{S F}(x) \frac{1}{16 x^{4}}\left(3-3 x-4 x t+2 x^{2} t\right),
\end{aligned}
$$

donde

$$
A^{S F}(x)=A_{0}^{S F} \mu_{p}^{2} \mathcal{Q}^{2} \alpha^{\prime 4} R^{2 p-6}\left(\frac{\Lambda^{2}}{q^{2}}\right)^{\gamma} x^{\gamma+2 n+5}(1-x)^{\gamma-1},
$$

Con la redefinición $W_{i}=\frac{1}{12 x^{3}} A^{S F}(x) \widetilde{W_{i}}$ poedmos ver la dependencia en $x$ a primer orden en $t$. Esta viene dada por

$$
\begin{array}{rll}
\widetilde{F_{1}}=(1-x) & , \quad \widetilde{F_{2}}=2(1-x), \\
\widetilde{b_{1}}=3(1-x) & , \quad \widetilde{b_{2}}=6(1-x) \\
\widetilde{b_{3}}=\frac{1}{2}(1-4 x) & , \quad \widetilde{b_{4}}=-(1-4 x), \\
\widetilde{g_{2}}=\frac{3}{4 x}(3-3 x) & , \quad \widetilde{g_{1}}=\frac{3 x t}{2}(-7+6 x) \approx 0 .
\end{array}
$$

Esto significa que encontramos en este régimen las relaciones

$$
F_{2}=2 F_{1}, b_{2}=2 b_{1}, b_{1}=3 F_{1}, g_{2}=\frac{9}{4 x} F_{1}, b_{4}=-2 b_{3},
$$

como ya se ha explicado en el Capítulo 6. Sustituyendo estas relaciones en la expresión general para espín 1 obtenemos

$$
\begin{aligned}
\frac{d \sigma}{d x d y d \phi} & =\frac{M E e^{4}}{4 \pi^{2} q^{4}}\left\{\langle q q\rangle\left[x y^{2}+2(1-y)\right] F_{1}+x y^{2} g_{2}\left[\frac{\left(s_{h} \cdot s_{l}\right)}{(P \cdot q)}-\frac{\left(q \cdot s_{h}\right)\left(P \cdot s_{l}\right)}{(P \cdot q)^{2}}\right]\right\} \\
& =\frac{M E e^{4} A^{S F}}{48 \pi^{2} q^{4} x^{3}}(1-x)\left\{\langle q q\rangle\left[x y^{2}+2(1-y)\right]+\frac{9}{4} y^{2}\left[\frac{\left(s_{h} \cdot s_{l}\right)}{(P \cdot q)}-\frac{\left(q \cdot s_{h}\right)\left(P \cdot s_{l}\right)}{(P \cdot q)^{2}}\right]\right\} .
\end{aligned}
$$

Para el caso del haz leptónico longitudinalmente polarizado, donde $s_{l}=H_{l} k$ con $H_{l}= \pm 1$ para helicidad positiva o negativa respectivamente, podemos reescribir esta expresión como

$$
\frac{d \sigma}{d x d y d \phi}=\frac{M E e^{4} A^{S F}}{48 \pi^{2} q^{4} x^{3}}(1-x)\left\{\langle q q\rangle\left[x y^{2}+2(1-y)\right]-\frac{9 H_{l}}{4 M E} s_{h}^{\mu}\left(y k_{\mu}-q_{\mu}\right)\right\} .
$$

Podemos estudiar el comportamiento de este resultado para cada estado de polarización. 
- Blanco no polarizado: $\langle q q\rangle_{\text {unpol }}=1, s_{h}=0$.

$$
\left.\frac{d \sigma}{d x d y d \phi}\right|_{\text {unpol }}=\frac{M E e^{4} A^{S F}}{48 \pi^{2} q^{4} x^{3}}(1-x)\left[x y^{2}+2(1-y)\right] .
$$

- Blanco longitudinalmente polarizado: $\langle q q\rangle_{L P}=0, s_{h}=\left(0, H_{h} M \hat{z}\right)(\mathrm{y}$ usando $\cos \theta \approx 1)$.

$$
\left.\frac{d \sigma}{d x d y d \phi}\right|_{L P}=0 \text {. }
$$

Este es el resultado a primer orden.

- Blanco transversalmente polarizado: $\langle q q\rangle_{T P}=\frac{3}{2}, s_{h}=\left(0, H_{h} M \hat{x}\right)$

$$
\left.\frac{d \sigma}{d x d y d \phi}=\frac{M E e^{4} A^{S F}}{48 \pi^{2} q^{4} x^{3}}(1-x)\left\{\frac{3}{2}\left[x y^{2}+2(1-y)\right]+H_{h} \frac{9 H_{l}}{4} \frac{M}{|q|}(1-y) \cos \phi\right\} 8.3 .7\right)
$$

Finalmente, para las amplitudes de helicidad en este caso obtenemos

$$
\begin{aligned}
A_{++,++} & \simeq 0+O(t) \\
A_{+0,+0} & \simeq 3 F_{1} \\
A_{+0,0+} & \simeq \sqrt{-t}\left[\frac{9 F_{1}}{2}+\frac{a_{3}}{2}+\frac{a_{4}}{4}\right]=\sqrt{-t} 6 F_{1} \\
A_{+-,+-} & \simeq 0+O(t) \\
A_{+-, 00} & \simeq \sqrt{-t}\left[\frac{9 F_{1}}{2}-\frac{a_{3}}{2}-\frac{a_{4}}{4}\right]=\sqrt{-t} 3 F_{1} \\
A_{+-,-+} & \simeq 0 \\
A_{0+, 0+} & \simeq \frac{4 b_{3}}{3 x} \\
A_{00,00} & \simeq 3\left(\frac{1}{x}-1\right) F_{1}-\frac{8 b_{3}}{3 x}
\end{aligned}
$$

8.3.2. Región B: $e^{-\sqrt{\lambda}} \ll x \ll 1 / \sqrt{\lambda}$ siones

Los resultados obtenidos en el Capítulo 6 para la región B se pueden resumir en las expre-

$$
\begin{array}{rlrl}
F_{1}=\frac{1}{12 x^{2}} I_{1} & , & F_{2}=\frac{1}{6 x}\left(I_{1}+I_{0}\right), \\
b_{1}=\frac{1}{4 x^{2}} I_{1} & , & b_{2}=\frac{1}{2 x}\left(I_{1}+I_{0}\right), \\
b_{3}=-\frac{1}{3 x}\left(I_{1}+I_{0}\right) & , & b_{4}=\frac{1}{6 x}\left(I_{1}+I_{0}\right), \\
g_{1}=0 & , \quad g_{2}=\frac{1}{8 x^{2}}\left(I_{1}+I_{0}\right),
\end{array}
$$

donde $I_{1} \sim q^{-2}$ y $1<\alpha \equiv 1+I_{0} / I_{1}<2$ son los únicos factores que dependen del modelo y de $l$, siendo $l$ el índice del armónico esférico en la solución de los mesones escalares y vectoriales. Estos resultados fueron expuestos y discutidos en el Capítulo 7, y dan lugar a las relaciones

$$
F_{2}=2 x F_{1}\left(1+\frac{I_{0}}{I_{1}}\right), b_{2}=2 x b_{1}\left(1+\frac{I_{0}}{I_{1}}\right), b_{4}=\frac{1}{3} b_{2}=-\frac{1}{2} b_{3} .
$$


Sustituyendo las funciones de estructura obtenidas por nosotros en la forma más general para la sección eficaz para espín 1 calculada en la Sección anterior tenemos

$$
\frac{d \sigma}{d x d y d \phi}=\frac{M E e^{4}}{48 \pi^{2} q^{4}} \frac{I_{1}}{x}\left\{\langle q q\rangle\left[y^{2}+2(1-y) \alpha\right]-\frac{3 \alpha}{M E} H_{l} s_{h}^{\mu}\left(y k_{\mu}-q_{\mu}\right)\right\}
$$

Estudiamos el comportamiento de esta según la polarización del hadrón

- Blanco no polarizado: $\langle q q\rangle_{\text {unpol }}=1, s_{h}=0$.

$$
\left.\frac{d \sigma}{d x d y d \phi}\right|_{\text {unpol }}=\frac{M E e^{4}}{48 \pi^{2} q^{4}} \frac{I_{1}}{x}\left[y^{2}+2 \alpha(1-y)\right]
$$

- Blanco longitudinalmente polarizado: $\langle q q\rangle_{L P}=0, s_{h}=(0, \pm M \hat{z})$.

$$
\left.\frac{d \sigma}{d x d y d \phi}\right|_{L P}=0
$$

- Blanco transversalmente polarizado: $\langle q q\rangle_{T P}=\frac{3}{2}, s_{h}=(0, \pm M \hat{x})$

$$
\frac{d \sigma}{d x d y d \phi}=\frac{M E e^{4}}{48 \pi^{2} q^{4}} \frac{I_{1}}{x}\left\{\frac{3}{2}\left[y^{2}+2(1-y) \alpha\right]-3 \alpha(1-y) \sqrt{-t} H_{l} H_{h} \cos \phi\right\} .
$$

donde en todos los casos definimos $C=\frac{e^{4} M E}{16 \pi q^{2}} I_{1}$.

También podemos obtener el resultado para las amplitudes de helicidad en esta región, a saber

$$
\begin{aligned}
A_{++,++} \simeq 0 & , & A_{+0,+0} \simeq 3 F_{1}, \\
A_{+0,0+} \simeq 0 & , & A_{+-,+-} \simeq 0 \\
A_{+-, 00}=O(\sqrt{-t}) \approx 0 & , & A_{+-,-+}=0 \\
A_{0+, 0+}=2(\alpha-1) F_{1}>0 & , & A_{00,00}=3(\alpha-1) F_{1}>0 .
\end{aligned}
$$

\subsection{Discusión de los resultados}

\section{Funciones de estructura}

Empezaremos por comparar la fenomenología de las funciones de estructura de los mesones. Cabe aclarar que esta comparación no es sencilla, ya que los datos experimentales de la estructura interna de los mesones es mucho más limitada que la existente para bariones, especialmente para la región cinemática de interés, donde $x$ es pequeño. Ha habido ciertos desarrollos fenomenológicos para el caso del pión como [90, 91, 92, 93, 94 pero todos ellos se basan en las funciones de estructura de valencia provenientes de experimentos de Drell-Yan de piones con blanco fijo, lo que implica que los datos provienen exclusivamente de la región $x \geq 0,2$. Es decir, estos resultados no pueden ser comparados con el comportamiento de la forma $x^{-1}$ o $x^{-2}$ encontrado en el Capítulo 7 para nuestras funciones de estructura, que es la parte relevante para el estudio de los momentos. 4 .

El punto de partida de nuestro trabajo es similar al de las referencias [47, 95, 96, 97, 98], e involucra técnicas similares a las usadas en aquellos trabajos. En ellos se ve un importante

\footnotetext{
${ }^{4}$ El pión o mesón $\pi$ corresponde al mesón escalar con $l=1$ en nuestros modelos holográficos duales al ser el mesón pseudoescalar más liviano.
} 
nivel de acuerdo con datos de DIS de bariones provenientes de los experimentos H1 y ZEUS en HERA. Las citadas referencias toman como base los cálculos para glueballs de [5], y nuestro trabajo es una extensión no trivial de aquel. El hecho de que los mesones escalares de nuestros cálculos se comporten de manera similar a los glueballs de [5] para todos los valores del parámetro de Bjorken $x$ es una buena indicación para la validez de nuestros resultados. En particular, se cumplen las relaciones de Callan-Gross en la misma forma que en [5] . Esperamos que nuestros resultados puedan verse testeados experimentalmente, cuando los experimentos con mesones alcancen el régimen cinemático de nuestros cálculos.

En las Figuras 8.1, 8.2 y 8.3 se muestran los gráficos de las funciones de estructura obtenidas en las regiones A y B (Capítulos 6 y 7 respectivamente, correspondientes a nuestros trabajos [1, 2, 3]) para mesones vectoriales en los tres modelos holográficos estudiados. Se muestran los gráficos de las funciones $F_{1}, F_{2}, b_{1}$ y $b_{2}$ para el caso de mesones vectoriales polarizado ${ }^{5}$ para los modelos D3D7, D4D8 $\overline{\mathrm{D} 8}$ y D4D6 $\overline{\mathrm{D} 6}$, y se grafican los mesones más livianos con $l=1,2,3$. Las constantes de normalización se eligieron para ajustar los momentos a los calculados con lattice QCD, como se describe más abajo. ${ }^{6}$

Todas las funciones de estructura graficadas poseen un comportamiento similar. Por un lado, la región $\mathbf{A}$ ( $x>0,2$ en los gráficos, aproximadamente), está dominada por una función con un pico alrededor de $x \simeq 0,6$, y tiende a cero a medida que $x \rightarrow 1$. Por otro lado, para $x$ pequeño (región B, aproximadamente $x<0,1$ en el gráfico) el comportamiento tipo campana deja de valer, siendo el dominante el correspondiente a las funciones de la forma $x^{-1}$ o $x^{-2}$ según el caso, que divergen al tender $x \rightarrow 0$. Las similitudes entre distintas funciones de estructura de un mismo modelo responden al hecho de que se cumplen las relaciones tipo Callan-Gross en cada sector, así como también $b_{i}=3 F_{i}$. La similitud entre modelos se debe a la universalidad ya mencionada de las funciones de estructura encontradas. Notamos que para $x$ pequeño las funciones de estructura son muy similares para los distintos valores $l=1,2,3$, mientras que para $x$ grande estas se hacen más pequeñas a medida que $l$ crece. Este comportamiento es más abrupto en los modelos D4D8 $\overline{\mathrm{D} 8}$ y $\mathrm{D} 4 \mathrm{D} 6 \overline{\mathrm{D} 6}$ que en el D3D7, en los que se observa un orden de magnitud de diferencia entre las funciones para $l=2$ y $l=1$ en el caso de $x$ grande.

\section{Momentos de las funciones de estructura}

Comparamos aquí los momentos de las funciones de estructura obtenidas en este trabajo con las del pión y el mesón $\rho$ que se encuentran en la literatura para lattice QCD [99]. Nos concentraremos en las funciones de estructura dependientes del espín para estos mesones. Los cálculos en la citada referencia fueron realizados con fermiones de Wilson para tres valores de la masa de los quarks, lo que permite a los autores extrapolar el cálclulo al caso quiral. Sumando las integrales calculadas en las regiones $\mathbf{A}$, B y $\mathbf{C}$ para los momentos de $F_{2}$ del pión y los momentos de $F_{1}$ del mesón $\rho$, utilizando un cutoff arbitrario para $x \rightarrow 0$ y ajustando las constantes numéricas frente a cada contribución fuimos capaces de ajustar los momentos de QCD $M_{i}\left(F_{2}\right)$ para el pión para $i=1,2,3$ y $M_{i}\left(F_{1}\right)$ para el mesón $\rho$ para $i=2,3,4$. Pueden verse los resultados del ajuste en las Tablas 8.1 y 8.2 respectivamente.

Como se muestra en las Tablas 8.1 y 8.2, encontramos discrepancias con respecto a los cálculos de lattice QCD del orden del $20 \%$ para nuestros resultados para el pión (mesón escalar con $l=1$ ) y para el mesón $\rho$ (mesón vectorial con $l=1$ ). Estos valores son compatibles con el hecho de que nuestros cálculos se realizan en el límite planar de $N \gg 1$, pues este es el

\footnotetext{
${ }^{5}$ Elegimos estos gráficos pues $b_{3}, b_{4}$ y $g_{2}$ son múltiplos de $b_{2}$ y $F_{1}$ respectivamente, mientras que $g_{1}$ es idénticamente cero. Todas estas relaciones son a primer orden en $t$.

${ }^{6}$ Omitimos la dependencia de estas constantes con $l$ pues solo disponemos de los momentos de las funciones de estructura con $l=1$ para comparar, correspondientes al mesón $\rho$.
} 


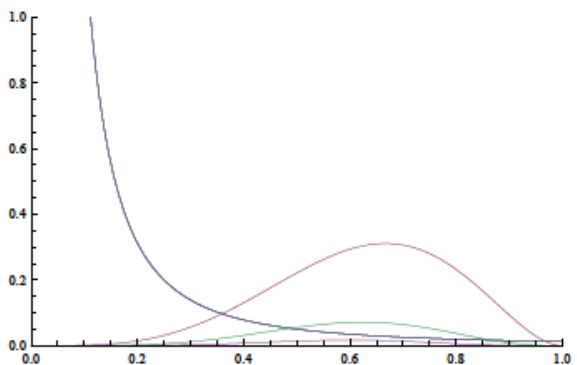

(a) $F_{1}(x)$

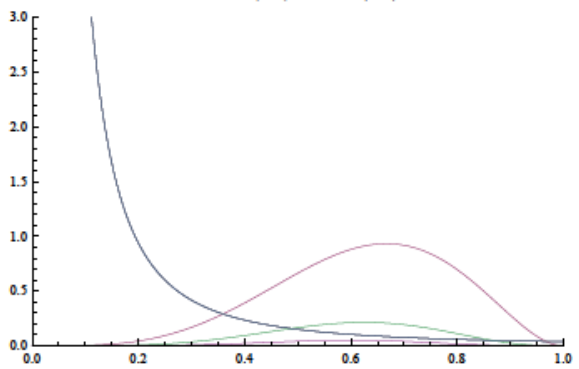

(c) $b_{1}(x)$

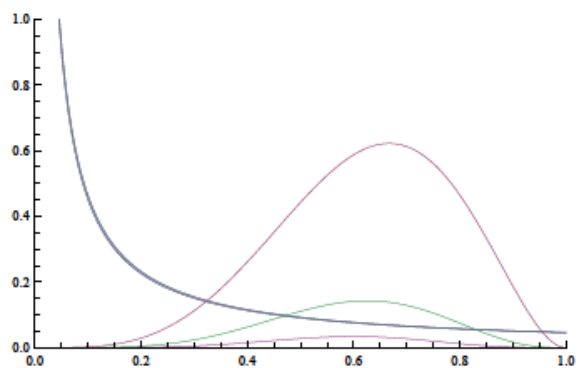

(b) $F_{2}(x)$

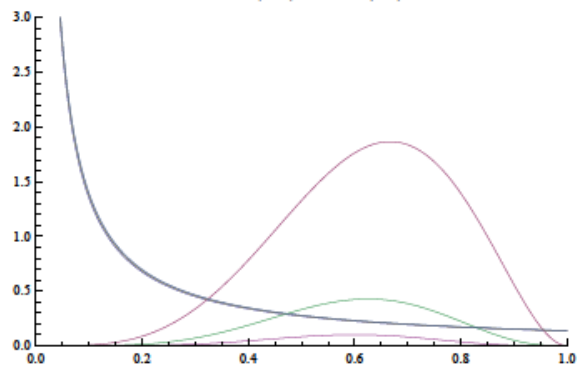

(d) $b_{2}(x)$

Figura 8.1: Funciones de estructura $F_{1}, F_{2}, b_{1}$ y $b_{2}$ en función del parámetro de Bjorken $x$ para mesones vectoriales en el modelo D3D7. Se grafican los tres modos más livianos: $l=1,2,3$.

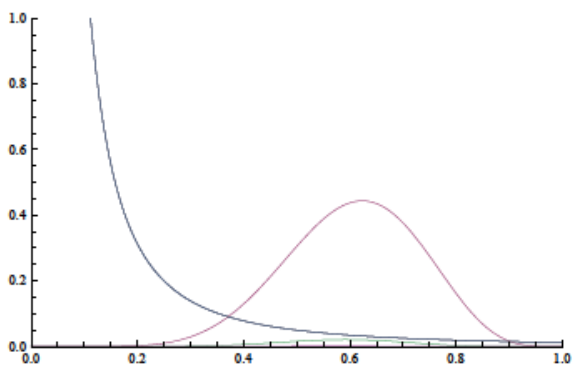

(a) $F_{1}(x)$

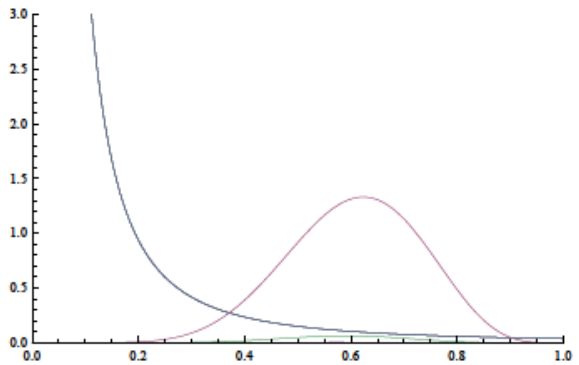

(c) $b_{1}(x)$

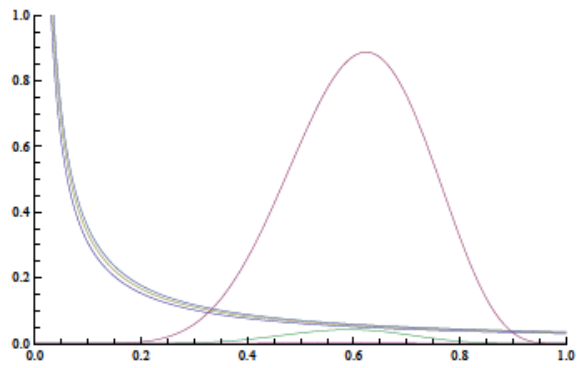

(b) $F_{2}(x)$

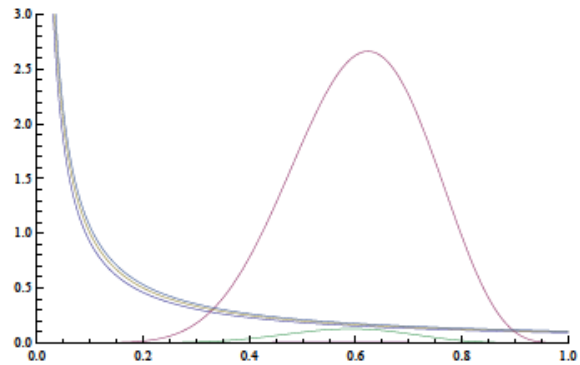

(d) $b_{2}(x)$

Figura 8.2: Funciones de estructura $F_{1}, F_{2}, b_{1}$ y $b_{2}$ en función del parámetro de Bjorken $x$ para mesones vectoriales en el modelo D4D8 $\overline{\mathrm{D} 8}$. Se grafican los tres modos más livianos: $l=1,2,3$. 


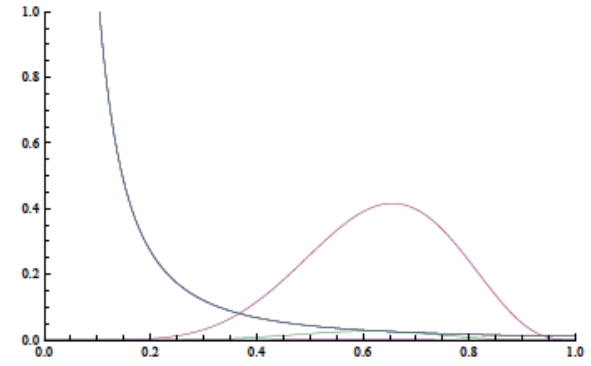

(a) $F_{1}(x)$

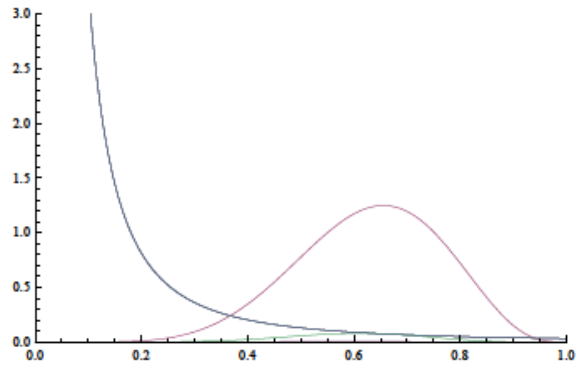

(c) $b_{1}(x)$

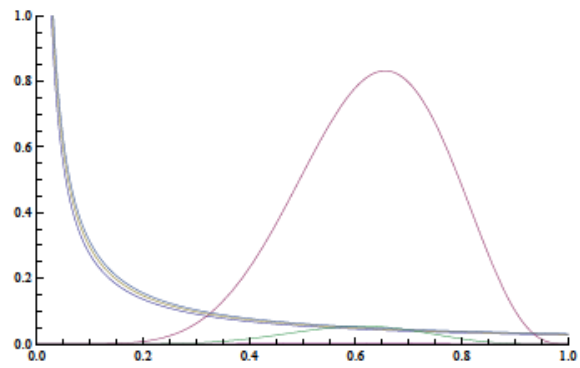

(b) $F_{2}(x)$

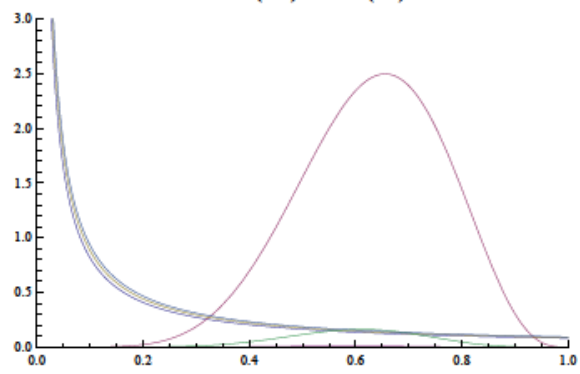

(d) $b_{2}(x)$

Figura 8.3: Funciones de estructura $F_{1}, F_{2}, b_{1}$ y $b_{2}$ en función del parámetro de Bjorken $x$ para mesones vectoriales en el modelo D4D6 $\overline{\mathrm{D} 6}$. Se grafican los tres modos más livianos: $l=1,2,3$.

\begin{tabular}{|c|c|c|c|}
\hline Modelo / Momento & $M_{1}\left(F_{2}\right)$ & $M_{2}\left(F_{2}\right)$ & $M_{3}\left(F_{2}\right)$ \\
\hline Lattice $Q C D$ & 0.3047 & 0.118 & 0.058 \\
\hline D3D7 & 0.3067 & 0.096 & 0.081 \\
\hline Diferencia porcentual & -0.6 & 18 & -13 \\
\hline $\mathrm{D} 4 \mathrm{D} 8 \overline{\mathrm{D} 8}$ & 0.3062 & 0.101 & 0.080 \\
\hline Diferencia porcentual & -0.5 & 15 & -10 \\
\hline $\mathrm{D} 4 \mathrm{D} 6 \overline{\mathrm{D} 6}$ & 0.3065 & 0.098 & 0.080 \\
\hline Diferencia porcentual & -0.6 & 17 & -12 \\
\hline
\end{tabular}

Tabla 8.1: Comparación de nuestros resultados para los primeros momentos de $F_{2}$ del mesón $\pi$ para valores de las constantes de normalización ajustados en base a los resultados promedio de los cálculos de [99] y comparación con estos. No se incluyen los errores para los resultados de lattice QCD. 


\begin{tabular}{|c|c|c|c|}
\hline Modelo / Momento & $M_{2}\left(F_{1}\right)$ & $M_{3}\left(F_{1}\right)$ & $M_{4}\left(F_{1}\right)$ \\
\hline Lattice $Q C D$ & 0.1743 & 0.074 & 0.035 \\
\hline D3D7 & 0.1755 & 0.059 & 0.040 \\
\hline Diferencia porcentual & -0.6 & 21 & -14 \\
\hline $\mathrm{D} 4 \mathrm{D} 8 \overline{\mathrm{D} 8}$ & 0.1752 & 0.062 & 0.040 \\
\hline Diferencia porcentual & -0.5 & 17 & -12 \\
\hline D4D6 $\overline{\mathrm{D} 6}$ & 0.1754 & 0.060 & 0.040 \\
\hline Diferencia porcentual & -0.6 & 19 & -13 \\
\hline
\end{tabular}

Tabla 8.2: Comparación de nuestros resultados para los primeros momentos de $F_{1}$ del mesón $\rho$ para valores de las constantes de normalización ajustados en base a los resultados promedio de los cálculos de [99] y comparación con estos. No se incluyen los errores para los resultados de lattice QCD.

error típico de tales cálculos. Por ejemplo, para los modelos bottom-up en cinco dimensiones los resultados de masas y constantes de decaimiento de mesones tienen resultados del orden de $5 \%$ [100, 101], mientras que para casos más complejos que involucran funciones de correlación de cuatro corrientes y que llevan a la regla $\Delta I=1 / 2$ que describe el decaimiento de kaones, las discrepancias son del orden del $30 \%$ [102, 103] con respecto a los experimentos.

Con respecto al mesón $\rho$, la forma de las funciones de estructura en la región A mostrada en las Figuras 8.1, 8.2 y 8.3 está de acuerdo con los resultados de [94].

Por otro lado, el las Figuras 8.4 y 8.5 se grafican, para el pión y el mesón $\rho$ respectivamente, los momentos obtenidos por nosotros con los tres modelos estudiados así como los resultados de [99]) obtenidos de lattice QCD con sus respectivas barras de error.

\section{Secciones eficaces}

En las Figuras 8.6 y 8.7 presentamos gráficos de las funciones de estructura totales en términos de $x$ e $y$, tanto para valores grandes como pequeños de $x$ y para los distintos modelos de $D p$-branas estudiados. Los colores más claros corresponden a valores mayores de la sección eficaz. 


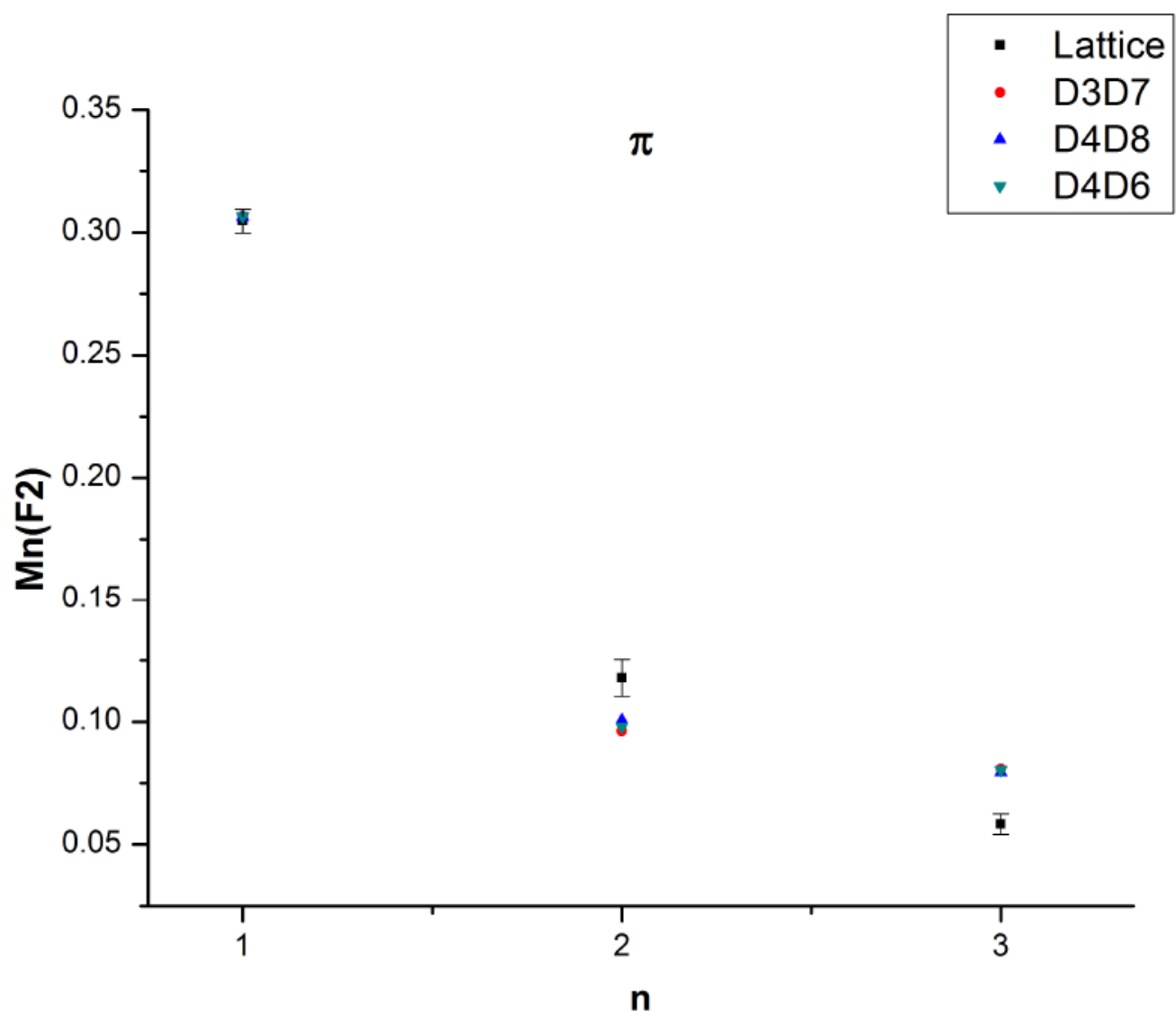

Figura 8.4: Momentos para el mesón $\pi$. Se muestran los tres primeros momentos para la función de estructura $F_{2}$ del mesón $\pi$. Se eligieron los parámetros libres para cada modelo holográfico dual de manera de ajustar a los valores obtenidos en [99] con métodos de lattice QCD. Se muestran los tres modelos holográficos duales así como el valor obtenido en la citada referencia con su error. Las discrepancias encontradas respecto de este valor de referencia no superan el $18 \%$ en ningún caso. 


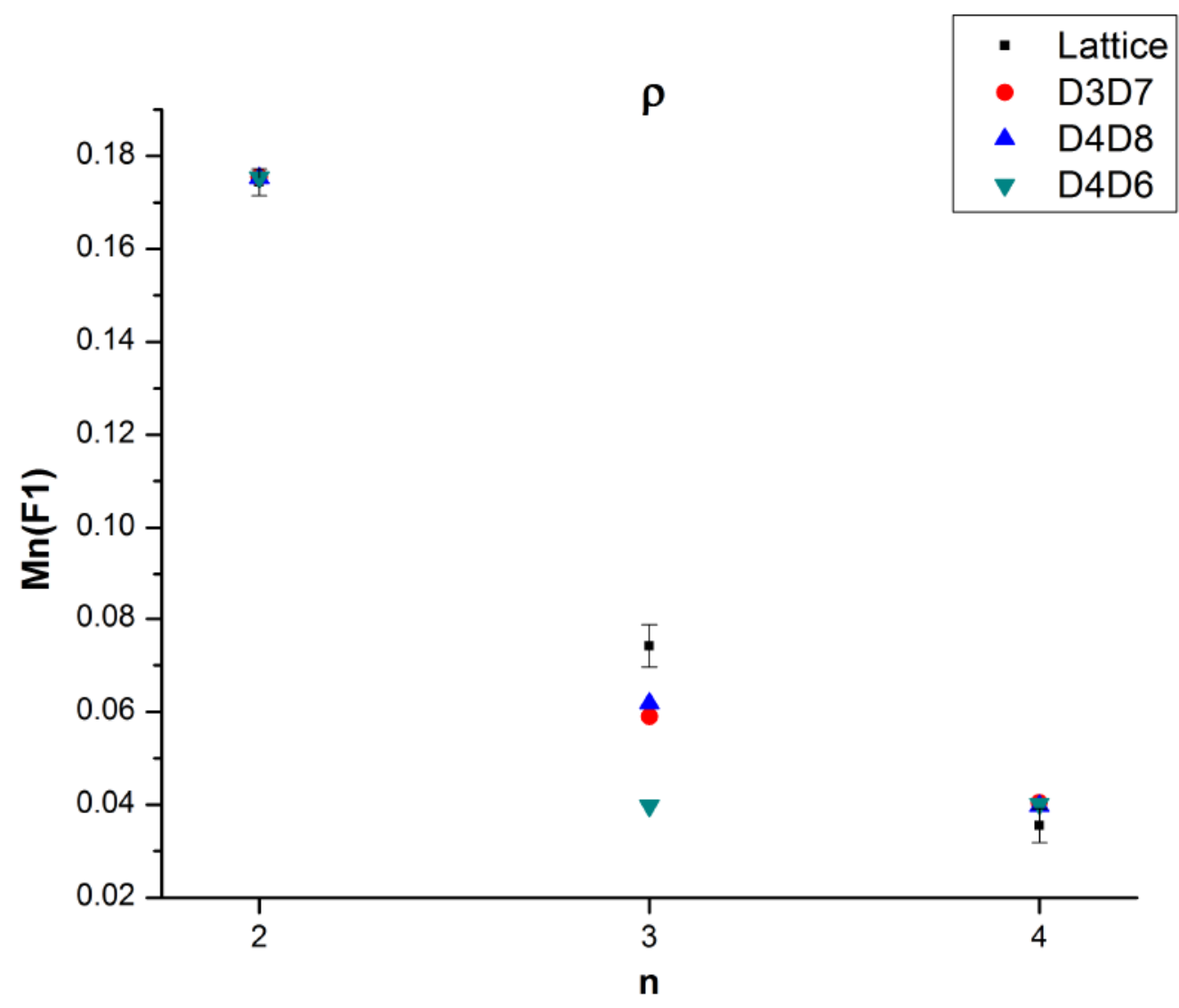

Figura 8.5: Momentos para el mesón $\rho$. Se muestran los tres primeros momentos para la función de estructura $F_{1}$ del mesón $\rho$. Se eligieron los parámetros libres para cada modelo holográfico dual de manera de ajustar a los valores obtenidos en [99] con métodos de lattice QCD. Se muestran los tres modelos holográficos duales así como el valor obtenido en la citada referencia con su error. Las discrepancias encontradas respecto de este valor de referencia no superan el $21 \%$ en ningún caso. 


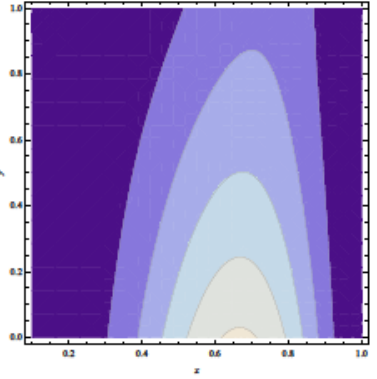

(a) $D 3 D 7 ; \quad \ell=1$

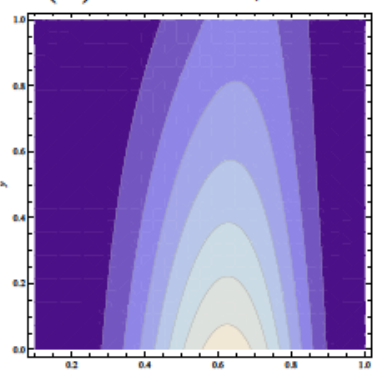

(d) $D 3 D 7 \quad ; \quad \ell=2$

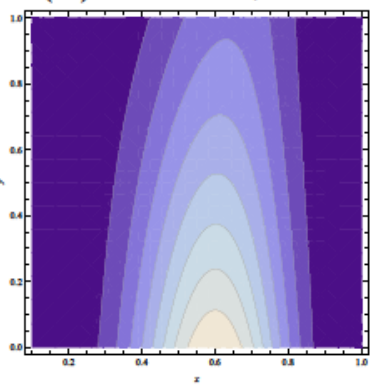

(g) $D 3 D 7 ; \quad \ell=3$

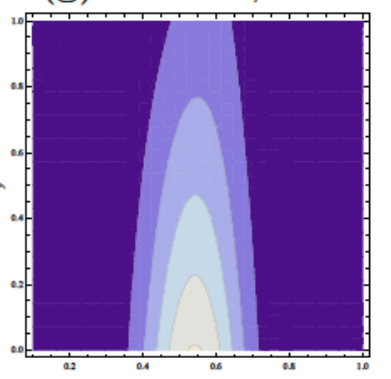

(j) $D 3 D 7 ; \quad \ell=10$

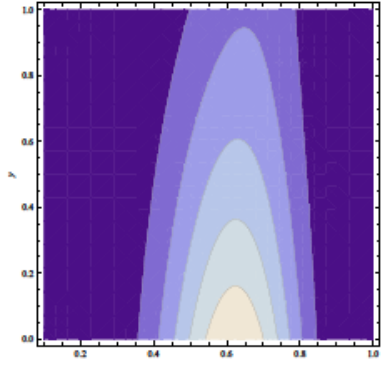

(b) $D 4 D 8 \overline{\mathrm{D} 8} \quad ; \quad \ell=1$

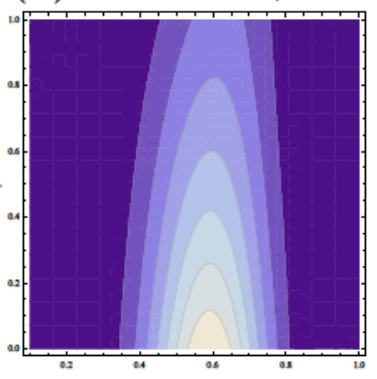

(e) $D 4 D 8 \overline{\mathrm{D} 8} \quad ; \quad \ell=2$

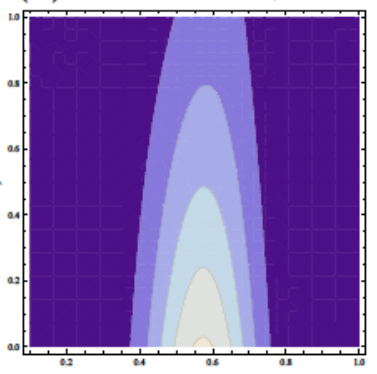

(h) $D 4 D 8 \overline{\mathrm{D} 8} \quad ; \quad \ell=3$

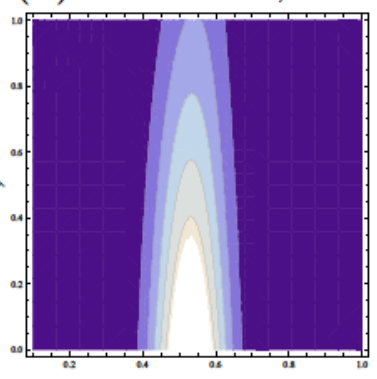

(k) $D 4 D 8 \overline{\mathrm{D} 8} ; \ell=10$

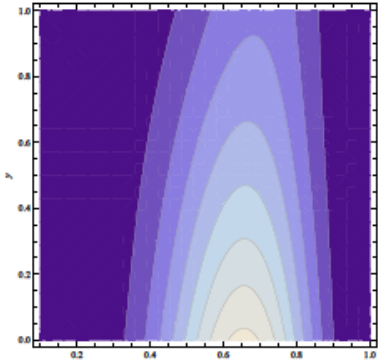

(c) $D 4 D 6 \overline{\mathrm{D} 6} ; \ell=1$

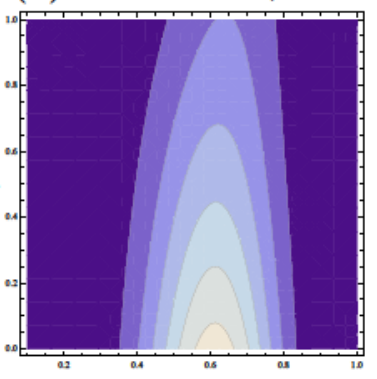

(f) $D 4 D 6 \overline{\mathrm{D} 6} \quad ; \quad \ell=2$

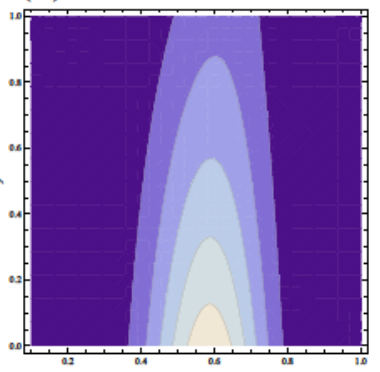

(i) $D 4 D 6 \overline{\mathrm{D} 6} ; \quad \ell=3$

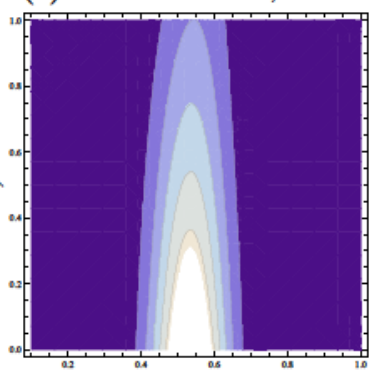

(l) $D 4 D 6 \overline{\mathrm{D} 6} ; \ell=10$

Figura 8.6: Curvas de nivel para la sección eficaz diferencial de DIS desde mesones vectoriales no polarizados para los tres modelos de $\mathrm{D} p$-branas estudiados en la región $\mathbf{A}$, correspondiente a $\frac{1}{\sqrt{\lambda}} \ll x<1$. El eje horizontal representa el parámetro de Bjorken $x$ en el rango $[0,1 ; 1]$ mientras que el eje vertical corresponde a la variable $y$ en el rango $[0 ; 1]$. Las zonas más oscuras corresponden a valores más bajos de la sección eficaz en cada figura. Se grafican $\operatorname{los} \operatorname{modos} l=1,2,3,10$. 


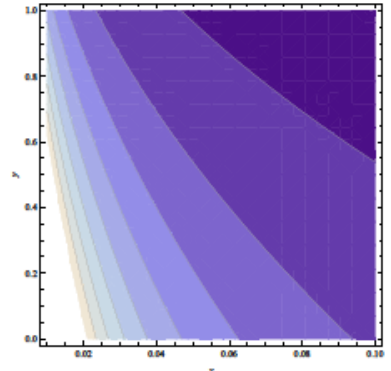

(a) $D 3 D 7 ; \ell=1$

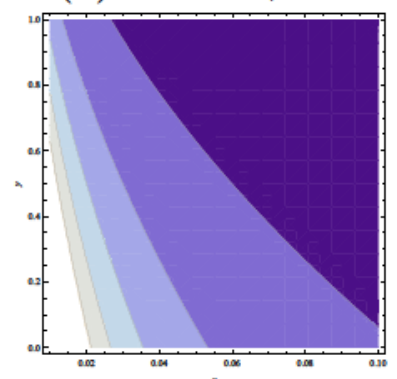

(d) $D 3 D 7 \quad ; \quad \ell=2$

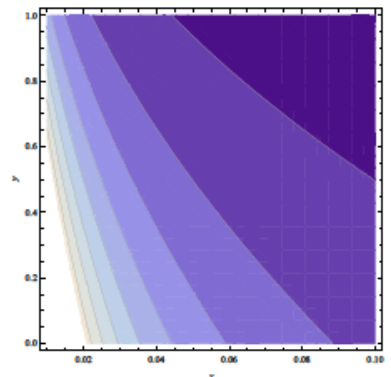

(g) $D 3 D 7 ; \quad \ell=3$

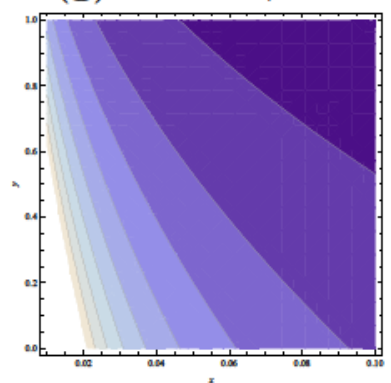

(j) $D 3 D 7 \quad ; \quad \ell=10$

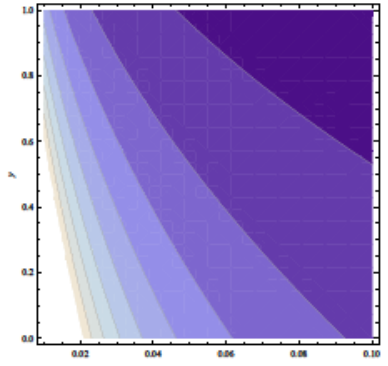

(b) $D 4 D 8 \overline{\mathrm{D} 8} \quad ; \quad \ell=1$

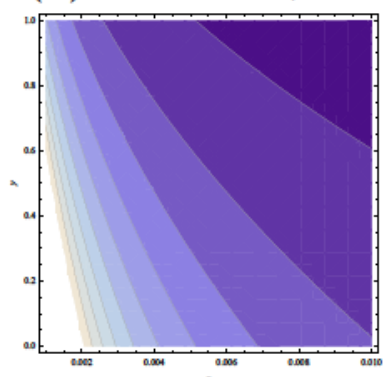

(e) $D 4 D 8 \overline{\mathrm{D} 8} \quad ; \quad \ell=2$

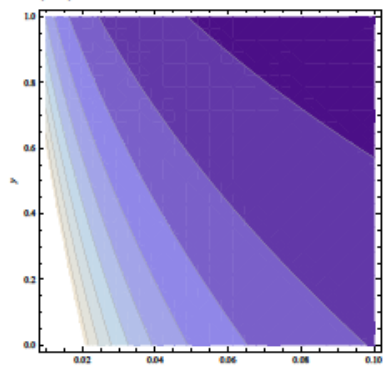

(h) $D 4 D 8 \overline{\mathrm{D} 8} \quad ; \quad \ell=3$

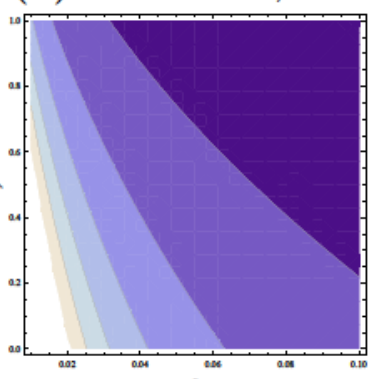

(k) $D 4 D 8 \overline{\mathrm{D} 8} ; \ell=10$

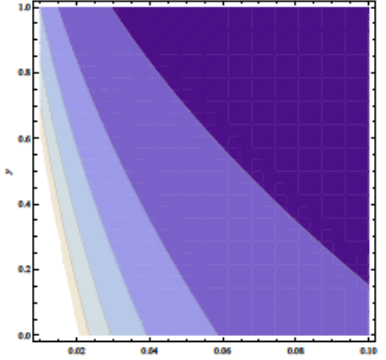

(c) $D 4 D 6 \overline{\mathrm{D} 6} \quad ; \quad \ell=1$

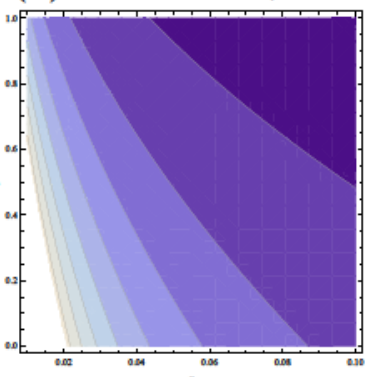

(f) $D 4 D 6 \overline{\mathrm{D} 6} \quad ; \quad \ell=2$

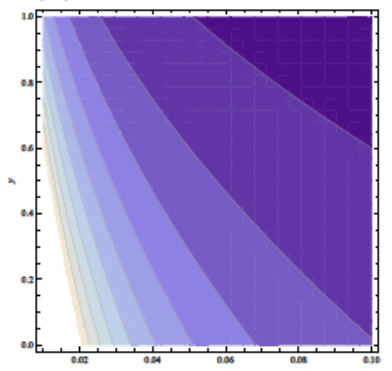

(i) $D 4 D 6 \overline{\mathrm{D} 6} \quad ; \quad \ell=3$

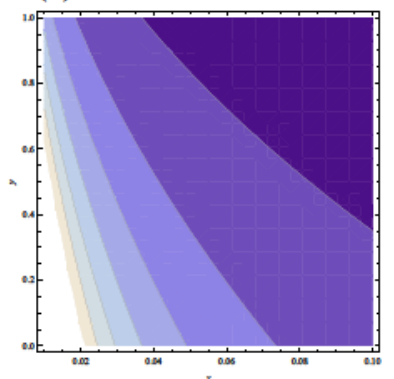

(l) $D 4 D 6 \overline{\mathrm{D} 6} ; \quad \ell=10$

Figura 8.7: Curvas de nivel para la sección eficaz diferencial de DIS desde mesones vectoriales no polarizados para los tres modelos de $\mathrm{D} p$-branas estudiados en la región $\mathbf{B}$, correspondiente a $e^{-\sqrt{\lambda}} \lesssim x \ll \frac{1}{\sqrt{\lambda}}$. El eje horizontal representa el parámetro de Bjorken $x$ en el rango $[0,001 ; 0,1]$ mientras que el eje vertical corresponde a la variable $y$ en el rango $[0 ; 1]$. Las zonas más oscuras corresponden a valores más bajos de la sección eficaz en cada figura. Se grafican los modos $l=1,2,3,10$. 


\section{Capítulo 9}

\section{Conclusiones}

Hemos estudiado en gran detalle el problema de la dispersión inelástica profunda DIS por mesones en el acoplamiento fuerte de teorías que poseen un modelo dual gravitatorio. En la región A de la Figura 1.1, correspondiente a $\frac{1}{\sqrt{\lambda}} \ll x<1$ realizamos el estudio con técnicas provenientes de supergravedad. En las regiones $\mathbf{B}$, rango en el que $e^{-\sqrt{\lambda}} \ll x \ll \frac{1}{\sqrt{\lambda}}$ y $\mathbf{C}$, $0 \ll e^{-\sqrt{\lambda}}$, lo realizamos con técnicas de teoría de cuerdas perturbativa. Cubrimos entonces todo el rango de valores para el parámetro de Bjorken $x$ en la zona de acoplamiento fuerte $\mathbf{y}$ límite planar de la teoría de gauge $1 \ll \lambda \ll N$. En todos los casos calculamos analíticamente las funciones de estructura para mesones vectoriales polarizados así como para mesones escalares. Encontramos relaciones novedosas del tipo Callan-Gross en todos los regímenes estudiados, con una independencia del modelo holográfico particular estudiado. Esto último sugiere una cierta universalidad en modelos provenientes de la dualidad gauge/gravedad en el acoplamiento fuerte y para $N$ grande, por lo que entendemos que, de encontrarse un dual gravitatorio para QCD, este tendría características similares a las aquí halladas en este rango.

En particular, para mesones vectoriales en la región $\mathbf{A}$ encontramos la relación $F_{2}=2 F_{1}$. Esta relación no coincide con la relación de Callan-Gross usual, a saber $F_{2}=2 x F_{1}$. La razón es que el factor $x$ que aparece en la relación usual representa la fracción del momento del hadrón incidente llevado por el partón que recibe la colisión. Como en el presente régimen el hadrón no se fragmenta, esta fracción es 1 y por ende no aparece en la relación de Callan-Gross encontrada por nosotros. También en este régimen encontramos la relación $b_{1}=3 F_{1}$, consistente con la relación $b_{1} \simeq \mathcal{O}\left(F_{1}\right)$ válida para el mesón $\rho$, compuesto de partones relativistas de espín $\frac{1}{2}$ [55]. También encontramos otras relaciones entre las funciones de estructura en este rango, a saber $b_{2}=2 b_{1}, b_{1}=3 F_{1}, g_{2}=\frac{9}{4 x} F_{1}$ y $b_{4}=-2 b_{3}$, además de encontrar sus expresiones analíticas para todos los valores de $x$ y $q^{2}$, siempre que se mantenga la aproximación de DIS $|t| \ll 1$. Los cálculos en esta región se desarrollaron con técnicas provenientes de supergravedad.

Para las regiones $\mathbf{B}$ y $\mathbf{C}$ también hemos encontrado relaciones tipo Callan-Gross. En particular, es interesante la relación $F_{2}=2 x \alpha F_{1}$, con $1 \leq \alpha \leq 2$ un parámetro dependiente de la dimensión de escala de cada mesón particular $\Delta$. Vemos cómo aquí aparece el factor $x$ que no estaba presente en la región anterior. También en esta región encontramos relaciones entre las funciones de estructura, específicamente $b_{2}=2 \alpha x b_{1}, b_{1}=3 F_{1}, b_{4}=-\frac{1}{2} b_{3}$ y $g_{1}=0$, así como su forma analítica para todos los valores de $x$ y $q^{2}$, en el rango cinemático permitido, con $|t| \ll 1$. Vale decir que los cálculos efectuados en esta región fueron altamente no triviales, dado que fue necesario utilizar técnicas complejas de teoría de cuerdas para llegar a ellos.

Por otro lado, estudiamos modelos con un número de D p-branas de prueba $N_{f}>1$, lo que implica una teoría de campos con $N_{f}$ sabores en la representación fundamental del grupo de 
gauge $S U(N)$, con lo que extrajimos las funciones de estructura para mesones con múltiples sabores. Encontramos que en la región A los mesones vectoriales provenientes de una teoría con $N_{f}>1$ y encontramos una expresión muy sencilla para relacionar sus funciones de estructura con las de mesones vectoriales con $N_{f}=1$, simplificando considerablemente la expresión para los primeros.

También hicimos un estudio de la expansión en loops de supergravedad, comprobando que estos solo tienen efecto del orden de potencias subdominantes de $\frac{1}{N}$ y $\frac{N_{f}}{N}$. Por último, realizamos un estudio de las secciones eficaces diferenciales en general para dispersión de mesones vectoriales con polarización arbitraria, y en particular para nuestros resultados en las tres regiones estudiadas.

Por otro lado, estudiamos los momentos de las funciones de estructura obtenidas y las comparamos con los datos de lattice QCD para los mesones más livianos en cada caso, a saber el pión ( $l=1$ pseudoescalar) y el mesón $\rho(l=1$ vectorial). Encontramos concordancia con nuestros cálculos para los tres modelos considerados dentro del error esperado para cálculos en el límite planar (del orden del $20 \%$ ) en todos los casos. Además, el comportamiento cualitativo de las funciones de estructura en todos los casos es el esperado.

Por último, se estudiaron las secciones eficaces de dispersión para distintos estados de polarización de los mesones vectoriales y se analizó la consistencia de estos cálculos. Exponemos estos resultados de secciones eficaces, así como los de los momentos de las funciones de estructura, como predicciones para experimentos ulteriores con mesones.

Quedan varias preguntas abiertas. Por un lado, sería deseable saber cómo se comportan estas funciones de estructura al disminuir el valor de $\lambda$ (moverse hacia la izquierda en la Figura 1.1). Esto corresponde a una expansión en $\alpha^{\prime} \sim \frac{1}{\sqrt{\lambda}}$. Por otro lado, sería deseable estudiar mayores órdenes en la expansión $\frac{1}{N}$ (moverse hacia fuera de la hoja en la Figura 1.1), lo que nos acercaría más a una teoría con $N$ finito como QCD. En este caso un cálculo inicial puede encararse con los lagrangianos efectivos derivados en [2] y la Sección 6.6 de esta Tesis. sin embargo, para desarrollar cálculos más precisos es encesario utilizar también diagramas de teoría de cuerdas considerando superficies de Riemann con genus $g>0$.

Otro aspecto interesante sería preguntarse por modelos holográficos duales con sabores sobre extensiones de los modelos de Klebanov-Strassler en el conifold [57, 59, 60, 61] y de MaldacenaNúñez [58, 62]. También se podrían desarrollar estudios similares para teorías en $2+1$ dimensiones desde el punto de vista formal, como por ejemplo en los casos de [104, [105] y [106], así como también en teorías con defectos [62. Por otro lado es interesante notar que el cálculo de funciones de correlación de dos corrientes ha sido de importancia en el contexto de los plasmas de quarks y gluones. En particular fotoemisión y conductividad eléctrica se han estudiado en [107] y sus correcciones en $\alpha^{\prime}$ en $[108,109,110,111,112$. 


\section{Apéndice A}

\section{Cálculo del factor $G$}

Utilizando la condición de cuerdas no masivas $\tilde{s}+\tilde{t}+\tilde{u}=0$ e identidades de las funciones gamma podemos escribir

$$
G=-\frac{\alpha^{\prime} \pi}{4 y^{2}} \frac{\sin [\pi(x+y)]}{\sin (\pi x) \sin (\pi y)}\left[\frac{\Gamma(x+y)}{\Gamma(x) \Gamma(y)}\right]^{2},
$$

donde definimos las variables

$$
x \equiv \frac{\alpha^{\prime} \tilde{s}}{4} \quad ; \quad y \equiv \frac{\alpha^{\prime} \tilde{t}}{4}
$$

En el régimen de interés $0 \leq \alpha^{\prime} \tilde{t} \ll 1 \ll \alpha^{\prime} \tilde{s}$, es decir $0 \leq y \ll 1 \ll x$ la forma asintótica de las funciones gamma resulta

$$
G=-\left[\frac{1}{\tilde{t}}+\frac{\pi \alpha^{\prime}}{4} \cot \left(\frac{\pi}{4} \alpha^{\prime} \tilde{s}\right)\right]\left(\frac{\alpha^{\prime} \tilde{s}}{4 e}\right)^{\frac{\alpha^{\prime} \tilde{t}}{2}}\left(1+O\left(\alpha^{\prime} \tilde{t}\right)\right)
$$

Si bien esta función $G$ es puramente real, posee infinitos polos, a saber $\tilde{t}=0$ y los polos de la función cotangente, que son los $\tilde{s}_{m}$ que cumplen $\frac{\pi}{4} \alpha^{\prime} \tilde{s}_{m}=m \pi$. Dada una función analítica arbitraria $f(\tilde{s}, \tilde{t})$, es claro que

$$
\begin{aligned}
\left.\sum_{\text {polos } p} \operatorname{Res}[G(\tilde{s}, \tilde{t}) f(\tilde{s}, \tilde{t})]\right|_{p} & =\left.\operatorname{Res}[G(\tilde{s}, \tilde{t}) f(\tilde{s}, \tilde{t})]\right|_{\tilde{t}=0}+\left.\sum_{m=0}^{\infty} \operatorname{Res}[G(\tilde{s}, \tilde{t}) f(\tilde{s}, \tilde{t})]\right|_{\tilde{s}_{m}} \\
& =\left.\operatorname{Res}[G(\tilde{s}, \tilde{t})]\right|_{\tilde{t}=0} f(\tilde{s}, 0)+\left.\sum_{m=0}^{\infty} \operatorname{Res}[G(\tilde{s}, \tilde{t})]\right|_{\tilde{s}_{m}} f\left(\tilde{s}_{m}, \tilde{t}\right) \\
& =-f(\tilde{s}, 0)-\frac{\pi \alpha^{\prime}}{4} \sum_{m=0}^{\infty} f\left(\tilde{s}_{m}, \tilde{t}\right)\left(\frac{\alpha^{\prime} \tilde{s}_{m}}{4 e}\right)^{\frac{\alpha^{\prime} \tilde{t}}{2}}
\end{aligned}
$$

donde Res denota el residuos de la función argumento en el punto indicado. Con esto, la integral 
de esta función serât

$$
\begin{aligned}
\int_{-\infty}^{+\infty} \int_{-\infty}^{+\infty} d \tilde{s} d \tilde{t} G(\tilde{s}, \tilde{t}) f(\tilde{s}, \tilde{t}) & =-\left.\frac{1}{2} 2 \pi i \sum_{\text {polos } p} \operatorname{Res}[G(\tilde{s}, \tilde{t}) f(\tilde{s}, \tilde{t})]\right|_{p} \\
& =\pi i\left[f(\tilde{s}, 0)+\frac{\pi \alpha^{\prime}}{4} \sum_{m=0}^{\infty} f\left(\tilde{s}_{m}, \tilde{t}\right)\left(\frac{m}{e}\right)^{\frac{\alpha^{\prime} \tilde{t}}{2}}\right]
\end{aligned}
$$

con lo cual

$$
G(\tilde{s}, \tilde{t})=\pi i\left[\delta(\tilde{t})+\frac{\pi \alpha^{\prime}}{4} \sum_{m=0}^{\infty} \delta\left(m-\frac{\alpha^{\prime} \tilde{s}}{4}\right)\left(\frac{m}{e}\right)^{\frac{\alpha^{\prime} \tilde{t}}{2}}\right]
$$

Quedándonos con la parte imaginaria y conservando solamente la parte que proviene del intercambio de estados de cuerdas excitadas, es decir no considerando los términos provenientes de $\tilde{s}=0$ y $\tilde{t}=0$, obtenemos

$$
\operatorname{Im}_{e x c}[G(\tilde{s}, \tilde{t})]=\frac{\pi \alpha^{\prime}}{4} \sum_{m=1}^{\infty} \delta\left(m-\frac{\alpha^{\prime} \tilde{s}}{4}\right)\left(\frac{m}{e}\right)^{\frac{\alpha^{\prime} \tilde{t}}{2}} .
$$

\footnotetext{
${ }^{1}$ Tomamos un contorno que vaya desde $-\infty$ hasta $+\infty$ en cada variable $(\tilde{s}, \tilde{t})$ esquivando las singularidades en sentido horario
} 


\section{Bibliografía}

[1] E. Koile, S. Macaluso and M. Schvellinger, "Deep Inelastic Scattering from Holographic Spin-One Hadrons," JHEP 1202, 103 (2012) [arXiv:1112.1459 [hep-th]].

[2] E. Koile, S. Macaluso and M. Schvellinger, "Deep inelastic scattering structure functions of holographic spin-1 hadrons with $N_{f} \geq 1$," JHEP 1401 (2014) 166 [arXiv:1311.2601 [hep-th]].

[3] E. Koile, N. Kovensky and M. Schvellinger, "Hadron structure functions at small $x$ from string theory," JHEP 1505, 001 (2015) [arXiv:1412.6509 [hep-th]].

[4] E. Koile, N. Kovensky and M. Schvellinger, "Deep inelastic scattering cross section from the gauge/string duality,". Work in preparation.

[5] J. Polchinski, M. J. Strassler, "Deep inelastic scattering and gauge / string duality," JHEP 0305 (2003) 012. [hep-th/0209211].

[6] C. Csaki, Y. Oz, J. Russo and J. Terning, "Large N QCD from rotating branes," Phys. Rev. D 59, 065012 (1999) [hep-th/9810186].

[7] C. Csaki, J. Russo, K. Sfetsos and J. Terning, "Supergravity models for (3+1)-dimensional QCD," Phys. Rev. D 60, 044001 (1999) [hep-th/9902067].

[8] S. S. Gubser, C. P. Herzog and I. R. Klebanov, "Symmetry breaking and axionic strings in the warped deformed conifold," JHEP 0409, 036 (2004) [hep-th/0405282].

[9] M. Schvellinger, "Glueballs, symmetry breaking and axionic strings in non-supersymmetric deformations of the Klebanov-Strassler background," JHEP 0409, 057 (2004) [hepth/0407152].

[10] E. Caceres and C.Núñez, "Glueballs of super Yang-Mills from wrapped branes," JHEP 0509, 027 (2005) [hep-th/0506051].

[11] J. Polchinski and M. J. Strassler, "The String dual of a confining four-dimensional gauge theory," hep-th/0003136.

[12] M. Kruczenski, D. Mateos, R. C. Myers, D. J. Winters, "Meson spectroscopy in AdS / CFT with flavor," JHEP 0307 (2003) 049. [arXiv:hep-th/0304032 [hep-th]].

[13] M. Kruczenski, D. Mateos, R. C. Myers, D. J. Winters, "Towards a holographic dual of large N(c) QCD," JHEP 0405 (2004) 041. [hep-th/0311270].

[14] T. Sakai, S. Sugimoto, "Low energy hadron physics in holographic QCD," Prog. Theor. Phys. 113 (2005) 843-882. [arXiv:hep-th/0412141 [hep-th]]. 
[15] N. Itzhaki, J. M. Maldacena, J. Sonnenschein and S. Yankielowicz, "Supergravity and the large N limit of theories with sixteen supercharges," Phys. Rev. D 58, 046004 (1998) [arXiv:hep-th/9802042].

[16] A. Chodos, R. L. Jaffe, K. Johnson and C. B. Thorn, "Baryon Structure in the Bag Theory," Phys. Rev. D 10, 2599 (1974).

[17] Y.Ñambu and G. Jona-Lasinio, "Dynamical Model of Elementary Particles Based on an Analogy with Superconductivity. 1.," Phys. Rev. 122, 345 (1961).

[18] Y.Ñambu and G. Jona-Lasinio, "Dynamical Model Of Elementary Particles Based On An Analogy With Superconductivity. Ii," Phys. Rev. 124, 246 (1961).

[19] T. H. R. Skyrme, "Particle states of a quantized meson field," Proc. Roy. Soc. Lond. A 262, 237 (1961).

[20] T. H. R. Skyrme, "A Nonlinear field theory," Proc. Roy. Soc. Lond. A 260, 127 (1961).

[21] T. H. R. Skyrme, "A Unified Field Theory of Mesons and Baryons," Nucl. Phys. 31, 556 (1962).

[22] J. B. Kogut, "A Review of the Lattice Gauge Theory Approach to Quantum Chromodynamics," Rev. Mod. Phys. 55, 775 (1983).

[23] J. M. Maldacena, "The large N limit of superconformal field theories and supergravity," Adv. Theor. Math. Phys. 2 (1998) 231 [Int. J. Theor. Phys. 38 (1999) 1113] [arXiv:hepth/9711200].

[24] E. Witten, "Anti-de Sitter space and holography," Adv. Theor. Math. Phys. 2 (1998) 253 [arXiv:hep-th/9802150].

[25] S. S. Gubser, I. R. Klebanov and A. M. Polyakov, "Gauge theory correlators from noncritical string theory," Phys. Lett. B 428 (1998) 105 [arXiv:hep-th/9802109].

[26] G. 't Hooft, "A Planar Diagram Theory for Strong Interactions," Nucl. Phys. B 72, 461 (1974).

[27] E. Witten, "Baryons in the 1/n Expansion," Nucl. Phys. B 160, 57 (1979).

[28] J. Polchinski, "Dirichlet Branes and Ramond-Ramond charges," Phys. Rev. Lett. 75, 4724 (1995) [hep-th/9510017].

[29] L. Susskind, "The World as a hologram," J. Math. Phys. 36, 6377 (1995) [hep-th/9409089].

[30] G. 't Hooft, "Dimensional reduction in quantum gravity," Salamfest 1993:0284-296 [grqc/9310026].

[31] L. Susskind and E. Witten, "The Holographic bound in anti-de Sitter space," hepth/9805114.

[32] O. Aharony, S. S. Gubser, J. M. Maldacena, H. Ooguri and Y. Oz, "Large N field theories, string theory and gravity," Phys. Rept. 323, 183 (2000) [hep-th/9905111].

[33] D. J. Gross and J. H. Sloan, "The Quartic Effective Action for the Heterotic String," Nucl. Phys. B 291 (1987) 41. 
[34] A. Sen and B. Zwiebach, "Tachyon condensation in string field theory," JHEP 0003, 002 (2000) [hep-th/9912249].

[35] B. Zwiebach, "Closed string field theory: Quantum action and the B-V master equation," Nucl. Phys. B 390, 33 (1993) [hep-th/9206084].

[36] C. G. Callan, Jr., E. J. Martinec, M. J. Perry and D. Friedan, "Strings in Background Fields," Nucl. Phys. B 262, 593 (1985).

[37] E. S. Fradkin and A. A. Tseytlin, "Effective Field Theory from Quantized Strings," Phys. Lett. B 158, 316 (1985).

[38] A. Sen, "Equations of Motion for the Heterotic String Theory from the Conformal Invariance of the Sigma Model," Phys. Rev. Lett. 55, 1846 (1985).

[39] C. G. Callan, Jr., I. R. Klebanov and M. J. Perry, "String Theory Effective Actions," Nucl. Phys. B 278, 78 (1986).

[40] M. T. Grisaru, A. E. M. van de Ven and D. Zanon, "Four Loop Divergences for the N=1 Supersymmetric Nonlinear Sigma Model in Two-Dimensions," Nucl. Phys. B 277, 409 (1986).

[41] S. P. de Alwis, "Strings in Background Fields, Beta Functions and Vertex Operators," Phys. Rev. D 34, 3760 (1986).

[42] J. Scherk, "Zero-slope limit of the dual resonance model," Nucl. Phys. B 31, 222 (1971).

[43] A. Neveu and J. Scherk, "Connection between Yang-Mills fields and dual models," Nucl. Phys. B 36, 155 (1972).

[44] J. Scherk and J. H. Schwarz, "Dual Models for Nonhadrons," Nucl. Phys. B 81, 118 (1974).

[45] T. Yoneya, "Connection of Dual Models to Electrodynamics and Gravidynamics," Prog. Theor. Phys. 51, 1907 (1974).

[46] M. B. Green, J. H. Schwarz and E. Witten, "Superstring Theory. Vol. 1: Introduction,"

[47] R. C. Brower, J. Polchinski, M. J. Strassler and C.-ITan, "The Pomeron and gauge/string duality," JHEP 0712 (2007) 005 [hep-th/0603115].

[48] M. Breidenbach, J. I. Friedman, H. W. Kendall, E. D. Bloom, D. H. Coward, H. C. DeStaebler, J. Drees and L. W. Mo et al., "Observed Behavior of Highly Inelastic electron-Proton Scattering," Phys. Rev. Lett. 23, 935 (1969).

[49] M. E. Peskin and D. V. Schroeder, "An Introduction to quantum field theory," Reading, USA: Addison-Wesley (1995) $842 \mathrm{p}$

[50] A. V. Manohar, "An Introduction to spin dependent deep inelastic scattering," [hep$\mathrm{ph} / 9204208]$.

[51] J. D. Bjorken and E. A. Paschos, "Inelastic Electron Proton and gamma Proton Scattering, and the Structure of the Nucleon," Phys. Rev. 185, 1975 (1969).

[52] J. D. Bjorken, "Asymptotic Sum Rules at Infinite Momentum," Phys. Rev. 179, 1547 (1969). 
[53] J. D. Bjorken, "Inequality for Backward electron-Nucleon and Muon-Nucleon Scattering at High Momentum Transfer," Phys. Rev. 163, 1767 (1967).

[54] J. D. Bjorken, "Inelastic Scattering of Polarized Leptons from Polarized Nucleons," Phys. Rev. D 1, 1376 (1970).

[55] P. Hoodbhoy, R. L. Jaffe, A. Manohar, "Novel Effects in Deep Inelastic Scattering from Spin 1 Hadrons," Nucl. Phys. B312 (1989) 571.

[56] A. Karch, E. Katz, "Adding flavor to AdS / CFT," JHEP 0206 (2002) 043. [arXiv:hepth/0205236 [hep-th]].

[57] I. R. Klebanov, M. J. Strassler, "Supergravity and a confining gauge theory: Duality cascades and chi SB resolution of naked singularities," JHEP 0008 (2000) 052. [arXiv:hepth/0007191 [hep-th]].

[58] J. M. Maldacena, C. Núñez, "Towards the large N limit of pure N=1 superYang-Mills," Phys. Rev. Lett. 86 (2001) 588-591. [hep-th/0008001].

[59] P. Ouyang, "Holomorphic D7 branes and flavored N=1 gauge theories," Nucl. Phys. B699 (2004) 207-225. [hep-th/0311084].

[60] S. Kuperstein, "Meson spectroscopy from holomorphic probes on the warped deformed conifold," JHEP 0503 (2005) 014. [hep-th/0411097].

[61] S. Kuperstein, J. Sonnenschein, "Analytic nonsupersymmtric background dual of a confining gauge theory and the corresponding plane wave theory of hadrons," JHEP 0402 (2004) 015. [hep-th/0309011].

[62] C. Núñez, A. Paredes, A. V. Ramallo, "Flavoring the gravity dual of N=1 Yang-Mills with probes," JHEP 0312 (2003) 024. [hep-th/0311201].

[63] C. G. Callan, Jr. and D. J. Gross, "High-energy electroproduction and the constitution of the electric current," Phys. Rev. Lett. 22, 156 (1969).

[64] C. G. Callan, Jr. and D. J. Gross, "Bjorken scaling in quantum field theory," Phys. Rev. D 8, 4383 (1973).

[65] C. G. Callan, Jr. and D. J. Gross, "Fixed Angle Scattering in Quantum Field Theory," Phys. Rev. D 11, 2905 (1975).

[66] C. G. Callan, Jr., R. F. Dashen and D. J. Gross, "The Structure of the Gauge Theory Vacuum," Phys. Lett. B 63, 334 (1976).

[67] J. Polchinski and M. J. Strassler, "Hard scattering and gauge / string duality," Phys. Rev. Lett. 88, 031601 (2002) [hep-th/0109174].

[68] R. Slansky, "Group Theory for Unified Model Building," Phys. Rept. 79, 1 (1981).

[69] Greiner, Reinhardt, "Field Quantization", Springer-Verlag Berlin Heidelberg, 1996.

[70] Misner, Thorne, Wheeler, "Gravitation", W. H. Freeman and Company, 1973.

[71] T. Sakai, J. Sonnenschein, "Probing flavored mesons of confining gauge theories by supergravity," JHEP 0309 (2003) 047. [hep-th/0305049]. 
[72] J. Babington, J. Erdmenger, N. J. Evans, Z. Guralnik, I. Kirsch, "Chiral symmetry breaking and pions in nonsupersymmetric gauge / gravity duals," Phys. Rev. D69 (2004) 066007. [hep-th/0306018].

[73] A. A. Tseytlin, "On nonAbelian generalization of Born-Infeld action in string theory," Nucl. Phys. B 501, 41 (1997) [hep-th/9701125].

[74] H. Liu and A. A. Tseytlin, "Dilaton - fixed scalar correlators and AdS(5) x S**5 - SYM correspondence," JHEP 9910 (1999) 003 [hep-th/9906151].

[75] D. Z. Freedman, S. D. Mathur, A. Matusis and L. Rastelli, "Correlation functions in the CFT(d) / AdS(d+1) correspondence," Nucl. Phys. B 546 (1999) 96 [hep-th/9804058].

[76] E. D'Hoker, S. D. Mathur, A. Matusis and L. Rastelli, "The Operator product expansion of N=4 SYM and the 4 point functions of supergravity," Nucl. Phys. B 589 (2000) 38 [hep-th/9911222].

[77] E. D'Hoker, D. Z. Freedman, S. D. Mathur, A. Matusis and L. Rastelli, "Graviton exchange and complete four point functions in the AdS / CFT correspondence," Nucl. Phys. B 562 (1999) 353 [hep-th/9903196].

[78] G. A. Miller, "Pionic and Hidden-Color, Six-Quark Contributions to the Deuteron b1 Structure Function," Phys. Rev. C 89, no. 4, 045203 (2014) [arXiv:1311.4561 [nucl-th]].

[79] J. B. Kogut and L. Susskind, "Scale invariant parton model," Phys. Rev. D 9 (1974) 697.

[80] J. R. Forshaw and D. A. Ross, "Quantum chromodynamics and the pomeron," Cambridge Lect. Notes Phys. 9, 1 (1997).

[81] H. D. I. Abarbanel, M. L. Goldberger and S. B. Treiman, "Asymptotic properties of electroproduction structure functions," Phys. Rev. Lett. 22, 500 (1969).

[82] K. Peeters, "A Field-theory motivated approach to symbolic computer algebra," Comput. Phys. Commun. 176 (2007) 550 [cs/0608005 [cs.SC]].

[83] K. Peeters, "Introducing Cadabra: A Symbolic computer algebra system for field theory problems," hep-th/0701238 [HEP-TH].

[84] S. Sannan, "Gravity as the Limit of the Type II Superstring Theory," Phys. Rev. D 34 (1986) 1749.

[85] S. Stieberger, "Open \& Closed vs. Pure Open String Disk Amplitudes," arXiv:0907.2211 [hep-th].

[86] A. Fotopoulos and A. A. Tseytlin, "On gravitational couplings in D-brane action," JHEP 0212 (2002) 001 [hep-th/0211101].

[87] A. Hashimoto and I. R. Klebanov, "Decay of excited D-branes," Phys. Lett. B 381 (1996) 437 [hep-th/9604065].

[88] C. Cheung, D. O'Connell and B. Wecht, "BCFW Recursion Relations and String Theory," JHEP 1009, 052 (2010) [arXiv:1002.4674 [hep-th]].

[89] A. Fotopoulos and N. Prezas, "Pomerons and BCFW recursion relations for strings on D-branes," Nucl. Phys. B 845, 340 (2011) [arXiv:1009.3903 [hep-th]]. 
[90] K. Wijesooriya, P. E. Reimer and R. J. Holt, "The pion parton distribution function in the valence region," Phys. Rev. C 72, 065203 (2005) [nucl-ex/0509012].

[91] R. J. Holt and C. D. Roberts, "Distribution Functions of the Nucleon and Pion in the Valence Region," Rev. Mod. Phys. 82, 2991 (2010) [arXiv:1002.4666 [nucl-th]].

[92] P. Reimer, R. Holt and K. Wijesooriya, "The Partonic Structure of the Pion at Large-x," AIP Conference Proceedings 1369, 153 (2011); doi: 10.1063/1.3631531

[93] L. Chang and A. W. Thomas, "Pion Valence-quark Parton Distribution Function," arXiv:1410.8250 [nucl-th].

[94] G. Altarelli, S. Petrarca and F. Rapuano, "The Pion structure function in a constituent model," Phys. Lett. B 373, 200 (1996) [hep-ph/9510346].

[95] R. C. Brower, M. J. Strassler and C. I. Tan, "On the eikonal approximation in AdS space," JHEP 0903, 050 (2009) [arXiv:0707.2408 [hep-th]].

[96] R. C. Brower, M. Djuric, I. Sarcevic and C. I. Tan, "String-Gauge Dual Description of Deep Inelastic Scattering at Small-x," JHEP 1011, 051 (2010) [arXiv:1007.2259 [hep-ph]].

[97] R. C. Brower, M. Djuric, I. Sarcevic and C. I. Tan, "Small-x Deep Inelastic Scattering via the Pomeron in AdS," arXiv:1204.0472 [hep-ph].

[98] R. Brower, R. C. Brower, M. Djuriat, T. Raben and C. I. Tan, "Towards holographic QCD: AdS/CFT, confinement deformation, and DIS at small-x," arXiv:1412.3443 [hep-ph].

[99] C. Best, M. Gockeler, R. Horsley, E. M. Ilgenfritz, H. Perlt, P. E. L. Rakow, A. Schafer and G. Schierholz et al., "Pion and rho structure functions from lattice QCD," Phys. Rev. D 56, 2743 (1997) [hep-lat/9703014].

[100] J. Erlich, E. Katz, D. T. Son and M. A. Stephanov, "QCD and a holographic model of hadrons," Phys. Rev. Lett. 95 (2005) 261602 [hep-ph/0501128].

[101] L. Da Rold and A. Pomarol, "Chiral symmetry breaking from five dimensional spaces," Nucl. Phys. B 721 (2005) 79 [hep-ph/0501218].

[102] T. Hambye, B. Hassanain, J. March-Russell and M. Schvellinger, "Four-point functions and Kaon decays in a minimal AdS/QCD model," Phys. Rev. D 76 (2007) 125017 [hep$\mathrm{ph} / 0612010]$.

[103] T. Hambye, B. Hassanain, J. March-Russell and M. Schvellinger, "On the Delta I = 1/2 rule in holographic QCD," Phys. Rev. D 74 (2006) 026003 [hep-ph/0512089].

[104] C. Núñez, I. Y. Park, M. Schvellinger and T. A. Tran, "Supergravity duals of gauge theories from F(4) gauged supergravity in six-dimensions," JHEP 0104, 025 (2001) [hepth/0103080].

[105] U. Gursoy, C.Ñunez and M. Schvellinger, JHEP 0206, 015 (2002) [hep-th/0203124].

[106] M. Schvellinger and T. A. Tran, "Supergravity duals of gauge field theories from SU(2) x U(1) gauged supergravity in five-dimensions," JHEP 0106, 025 (2001) [hep-th/0105019].

[107] S. Caron-Huot, P. Kovtun, G. D. Moore, A. Starinets and L. G. Yaffe, "Photon and dilepton production in supersymmetric Yang-Mills plasma," JHEP 0612, 015 (2006) [hepth/0607237]. 
[108] B. Hassanain and M. Schvellinger, "Holographic current correlators at finite coupling and scattering off a supersymmetric plasma," JHEP 1004, 012 (2010) [arXiv:0912.4704 [hepth]].

[109] B. Hassanain and M. Schvellinger, "Towards 't Hooft parameter corrections to charge transport in strongly-coupled plasma," JHEP 1010, 068 (2010) [arXiv:1006.5480 [hep-th]].

[110] B. Hassanain and M. Schvellinger, "Plasma conductivity at finite coupling," JHEP 1201, 114 (2012) [arXiv:1108.6306 [hep-th]].

[111] B. Hassanain and M. Schvellinger, "Diagnostics of plasma photoemission at strong coupling," Phys. Rev. D 85, 086007 (2012) [arXiv:1110.0526 [hep-th]].

[112] B. Hassanain and M. Schvellinger, "Plasma photoemission from string theory," JHEP 1212, 095 (2012) [arXiv:1209.0427 [hep-th]]. 


\section{Agradecimientos}

En este momento usted posee en sus manos diez metros cuadrados de papel de tamaño A4, con un peso total estimado de ochocientos gramos más encuadernación, en el que se expone el trabajo del autor durante el último lustro. Este no habría sido posible sin la colaboración de una gran cantidad de personas e instituciones que pasan a detallarse a continuación.

\section{Sobre este trabajo}

Quiero citar en primer lugar a Martín, quien no solo me guió durante todo el trayecto, sino que también con su empuje y dedicación logró que esta Tesis alcance el nivel final que aquí se presenta.

A Sebastián Macaluso y Nicolás Kovensky, que fueron coautores secuenciales de los trabajos que aquí se presentan y con quienes trabajé día a día durante el desarrollo de esta Tesis, en dos etapas bien distintas de esta.

Al Instituto de Física La Plata (IFLP - CCT La Plata - CONICET) y al Departamento de Física de la Facultad de Ciencias Exactas de la Universidad Nacional de La Plata (DF-FCEUNLP), donde esta Tesis fue desarrollada.

A la Agencia Nacional de Promoción Científica y Tecnológica por la beca con la que comencé este trabajo y por el subsidio PICT-2007-00849 del que formé parte. Al CONICET por la beca con la que finalicé este trabajo y el subsidio PIP-0396 del que formo parte.

Al Departamento de Física de la Facultad de Ciencias Exactas y Naturales de la Universidad de Buenos Aires (UBA), así como al Departamento de Ciencias Exactas del Ciclo Básico Común, donde fui docente a dos tiempos y pude ejercer la otra faceta de mi vocación académica.

Al Abdus Salam International Centre for Theoretical Physics (ICTP) por la financiación para viajar a diversas escuelas. Al Weizmann Institute of Science, la Universidade do Porto, el Centro Atómico Bariloche y el ICTP por permitirme visitarlos y dar seminarios sobre mi trabajo. A la Asociación Física Argentina (AFA), la Red Strings@ar y el IFLP, por organizar reuniones científicas frecuentes e invitarme a dar seminarios.

A José Goity (Jefferson Lab, EUA), Carlos Núñez (Swansea University, R.U.), Martín Kruczenski (Purdue University, EUA), Rodolfo Sassot (UBA) y Horacio Falomir (UNLP) por importantes discusiones durante el desarrollo de los distintos trabajos.

A los miembros del grupo, tanto de Buenos Aires como de La Plata (Gastón, Guille, Osvaldo, Diego, Nico G, Marcelo), por interesantes discusiones, tanto de física como de las otras.

Al grupo "local" (Martín, Sebastián, Nico K, David, Walter, Damián, Gus, Nacho y Santiago) por estar a mano para las discusiones.

A los desarrolladores de TeXmaker por hacer que esta tesis compile. A Jorge Cham por acompañarme desde su web. A Google Calendar y Trello por ayudar a organizar mi vida.

A la Real Academia Española y la Asociación de Academias de la Lengua Española por permitir el ahorro de considerable cantidad de tinta en la impresión de esta Tesis al abolir gran cantidad de tildes diacríticas en su Ortografía 2010. Tinta que, empero, fue derrochada con creces durante la impresión del presente párrafo. 


\section{Sobre la vida transcurrida en paralelo}

A los amigos de siempre y a los más nuevos: Iván, Santi, Nahuel, Pablito, Seba, Gustavo, Vero, Sofi, Mati, Marielita, Gabi, Vale, Sol, Meli... A los IB-éricos, exactos, bríos y tantos otros de los que me estaré olvidando.

A la Asociación Argentina Amigos de la Astronomía, donde me tratan como en casa, y en particular a la redacción de la Revista Astronómica.

Al Club Cultural Matienzo, por estar siempre para sorprender. A CouchSurfing, por permitirme conocer tanta gente y tantos lugares nuevos. A Chiri y el Gordo, por enseñarme a leer. A Irina por enseñarme a actuar. A Mónica por ayudarme a pensar. A Oster, por despertarme cada mañana. A los diversos hostels de la capital bonaerense por alojarme ocasionalmente.

Esta tesis fue escrita entre los meses de enero y marzo de 2015 en el Instituto de Física La Plata, la Facultad de Ciencias Exactas y Naturales de la Universidad de Buenos Aires, la Biblioteca del Congreso de la Nación, la cafetería Starbucks de Callao y Viamonte y el departamento de quien suscribe. 